\title{
The interplay between the genome and the exposome in psychosis spectrum
}

Citation for published version (APA):

Pries, L. (2020). The interplay between the genome and the exposome in psychosis spectrum. [Doctoral Thesis, Maastricht University]. Ridderprint. https://doi.org/10.26481/dis.20201211/p

Document status and date:

Published: 01/01/2020

DOI:

10.26481/dis.20201211/p

Document Version:

Publisher's PDF, also known as Version of record

\section{Please check the document version of this publication:}

- A submitted manuscript is the version of the article upon submission and before peer-review. There can be important differences between the submitted version and the official published version of record.

People interested in the research are advised to contact the author for the final version of the publication, or visit the DOI to the publisher's website.

- The final author version and the galley proof are versions of the publication after peer review.

- The final published version features the final layout of the paper including the volume, issue and page numbers.

Link to publication

\footnotetext{
General rights rights.

- You may freely distribute the URL identifying the publication in the public portal. please follow below link for the End User Agreement:

www.umlib.nl/taverne-license

Take down policy

If you believe that this document breaches copyright please contact us at:

repository@maastrichtuniversity.nl

providing details and we will investigate your claim.
}

Copyright and moral rights for the publications made accessible in the public portal are retained by the authors and/or other copyright owners and it is a condition of accessing publications that users recognise and abide by the legal requirements associated with these

- Users may download and print one copy of any publication from the public portal for the purpose of private study or research.

- You may not further distribute the material or use it for any profit-making activity or commercial gain

If the publication is distributed under the terms of Article $25 \mathrm{fa}$ of the Dutch Copyright Act, indicated by the "Taverne" license above, 


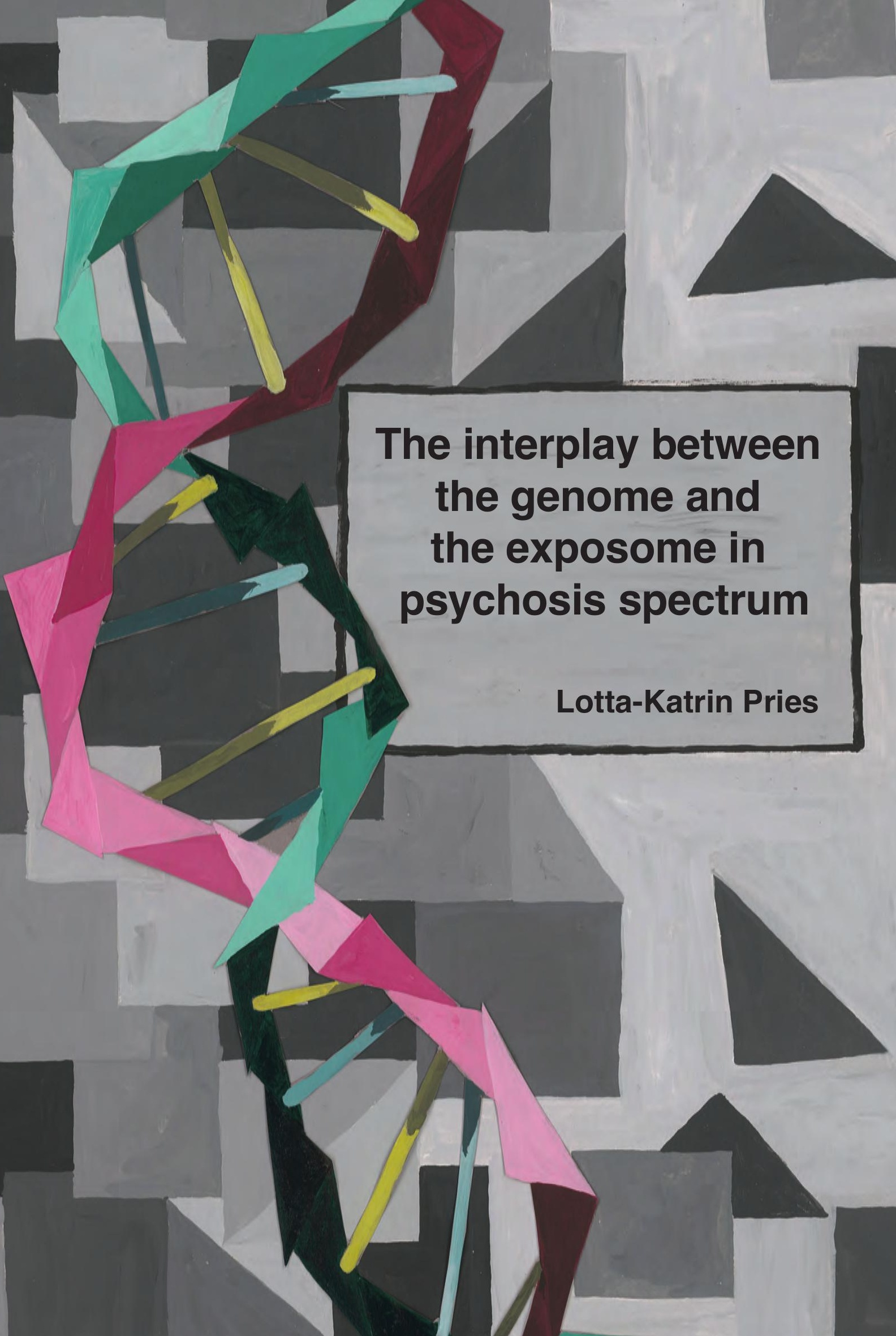



The interplay between the genome and the exposome in psychosis spectrum

Lotta-Katrin Lisa Katarina Paula Pries 
(C) copyright Lotta-Katrin Pries, Maastricht 2020

Print: Ridderprint | www.ridderprint.nl

Cover Design: Charlotte Pries

ISBN 9789464230611 


\title{
The interplay between the genome and the exposome in psychosis spectrum
}

\author{
DISSERTATION \\ to obtain the degree of Doctor \\ at the Maastricht University, \\ on the authority of the Rector Magnificus, \\ Prof. dr. Rianne M. Letschert \\ in accordance \\ with the decision of the Board of Deans, \\ to be defended in public \\ on Friday 11 December 2020, at 16:00 hours \\ by \\ Lotta-Katrin Lisa Katarina Paula Pries
}




\section{Supervisors}

Prof. Dr. B.P.F. Rutten

Prof. Dr. J. van Os

\section{Co-supervisor \\ Dr. S. Guloksuz}

\section{Assessment Committee}

Prof. Dr. Daniel van den Hove (chair)

Prof. Dr. Jacqueline Strik

Prof. Dr. Koen Schruers

Prof. Dr. Richard Bruggeman, Rijksuniversiteit Groningen

Prof. Dr. Wiepke Cahn, Universiteit Utrecht

The research presented in this thesis was conducted at the School for Mental Health and Neuroscience, Department of Psychiatry and Neuropsychology of Maastricht University, Maastricht University Medical Centre, Maastricht, the Netherlands 


\section{Contents}

Chapter 1 General Introduction 7

Chapter 2 Association of preceding psychosis risk states and non-

psychotic mental disorders with incidence of clinical

psychosis in the general population: a prospective study

in the NEMESIS-2 cohort

Chapter 3 Evidence that environmental and familial risks for

psychosis additively impact a multidimensional

subthreshold psychosis syndrome

Chapter 4 TwinssCan - gene-environment interaction in psychotic and depressive intermediate phenotypes: risk and protective factors in a general population twin sample

Chapter 5 White Noise Speech Illusion and Psychosis Expression: An experimental Investigation of Psychosis Liability

Chapter 6 Polygenic liability for schizophrenia and childhood adversity influences daily-life emotion dysregulation and psychosis proneness

Chapter 7 Examining the independent and joint effects of molecular genetic liability and environmental exposures in schizophrenia: results from the EUGEI study

Chapter 8 Estimating exposome score for schizophrenia using predictive modeling approach in two independent samples: the results from the EUGEI study

Chapter 9 Association of recent stressful life events with mental and physical health in the context of genomic and exposomic liability for schizophrenia

Chapter 10 Examining the independent and joint effects of genomic and exposomic liabilities for schizophrenia across the psychosis spectrum

Chapter 11 General Discussion

12.1 Resilience against traumatic stress: current developments and future directions

12.2 Summary

12.3 Impact paragraph

12.4 Acknowledgment

12.5 Curriculum Vitae

12.6 List of Publications 



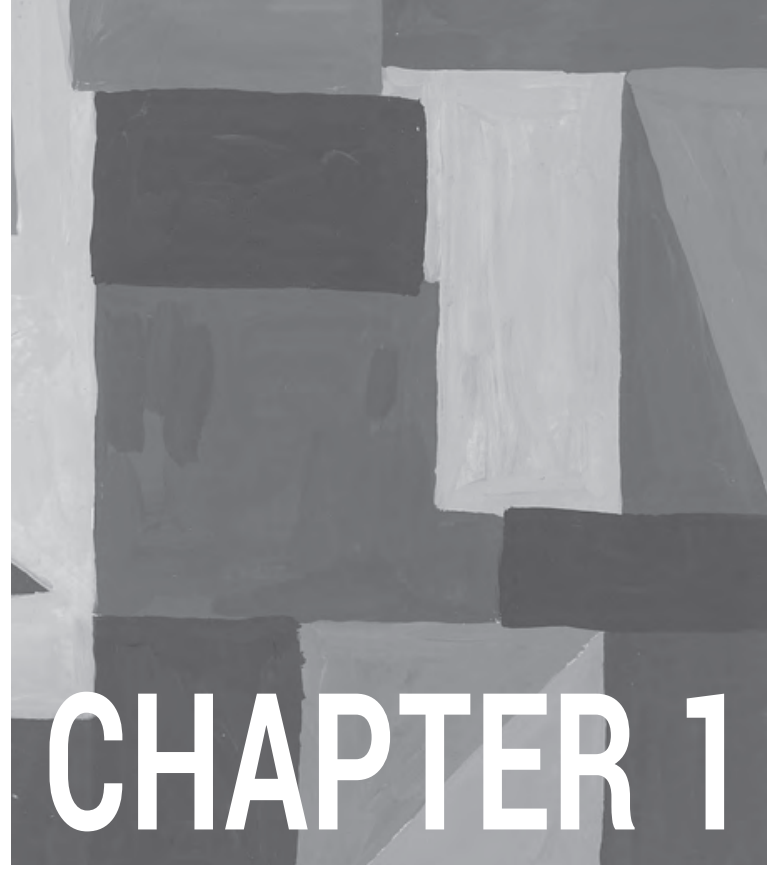

GENERAL INTRODUCTION

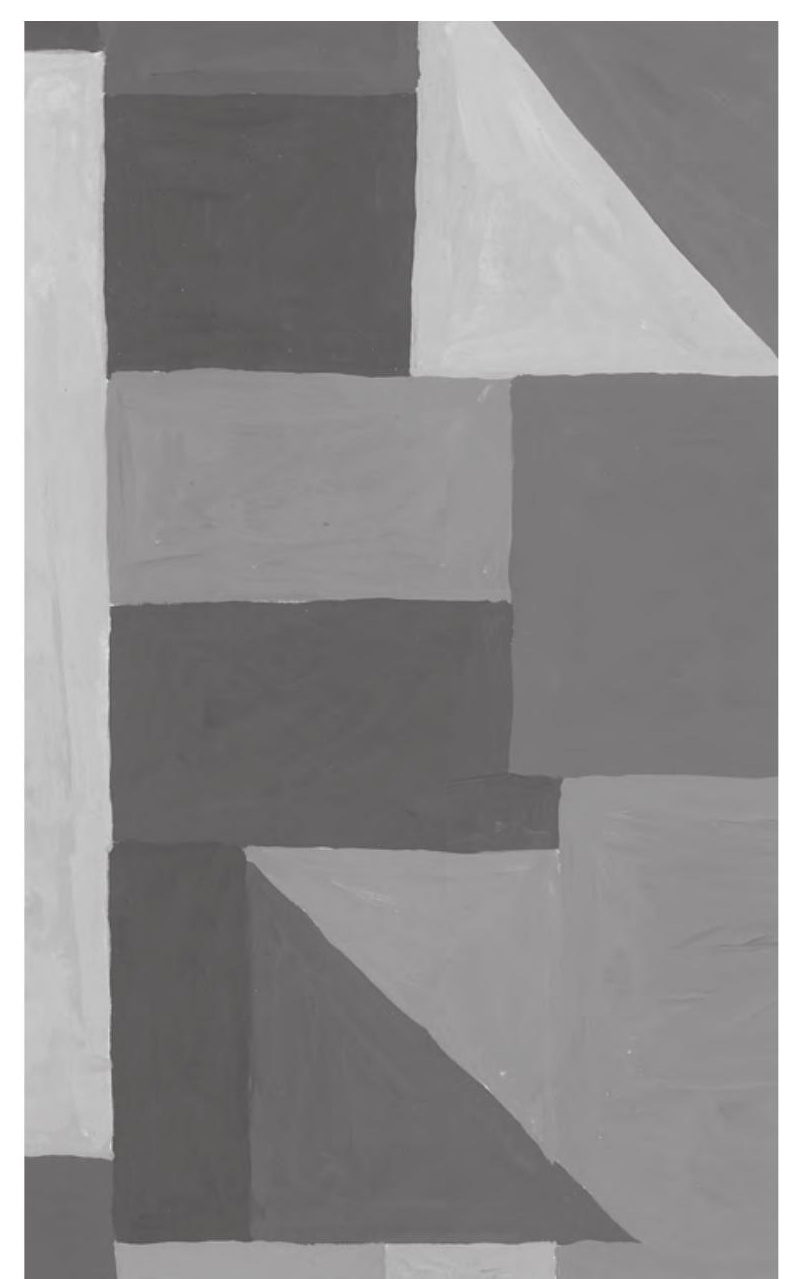




\section{From "schizophrenia" to "psychosis spectrum disorder"}

DSM-5 defines schizophrenia as a mental disorder with characteristic symptoms including delusions, hallucinations, disorganized speech, grossly disorganized or catatonic behavior, and negative symptoms, that is, diminished emotional expression or avolition ${ }^{1}$. However, likewise the narrow nosology as well as the terminology are regarded critically ${ }^{2}$. Schizophrenia as it is currently defined by the DSM-5 only captures the top 30\% most severe outcomes among psychosis disorder ${ }^{3}$. The remaining $70 \%$ are commonly ignored in academic literature ${ }^{4}$. Subtle psychosis expressions are highly prevalent and bidirectionally associated with other major mental disorders such as anxiety or depression ${ }^{5}$. Although the course of psychosis psychopathology is divers, the current categorical diagnoses do not embrace the different symptom profiles ${ }^{6-9}$. Despite great efforts, the mechanisms of schizophrenia remain poorly understood, which is greatly affected by the categorical diagnostics ${ }^{2,10}$. Therefore, next to the mainly unchanged categorical classification within DSM- $5^{11}$, a conceptual part on a broader psychosis spectrum concept was added to the chapter "Schizophrenia Spectrum and Other Psychotic Disorders" from DSM-IV to DSM-5. An appeal was made for further research. Following this, the recent years saw extensive work on the reconceptualization of psychosis phenotypes.

Researchers argue that psychosis phenotypes are better summarized and conceptualized in psychotic spectrum disorder (PSD) with a spectrum of severity stretching along a continuum from mild but persistent schizotypy, on the one hand, to the diagnosis of schizophrenia as the most severe outcome on the other hand ${ }^{2}$. The spectrum of psychosis phenotypes is expressed as a concordance of multidimensional psychopathology including negative symptoms, affective dysregulation and cognitive impairment, which vary in symptom severity and symptom pattern ${ }^{2,7,12}$. Clinical features traverse diagnostic categories ${ }^{12}$, and increased co-occurrence of psychotic and non-psychotic symptom dimensions is associated with decreased treatment response, decreased functioning, and poor outcome ${ }^{13-20}$. Initiatives such as the transdiagnostic Research Domain Criteria (RDoC) framework of the National Institute of Mental Health (NIMH) ${ }^{21}$ move away from the pragmatic categorical approach and prioritize an evidence-based dimensional conceptualization of psychosis phenotypes. The current thesis presents several methods to investigate multidimensional psychopathology along the severity spectrum in the general population and clinical samples.

\section{Psychosis expression within the network of psychopathology}

Evidence suggests that specialized early interventions for first-episode psychosis substantially improves short-term treatment outcome ${ }^{22}$. Furthermore, shortening the duration of untreated psychosis might improve long-term prognosis ${ }^{23}$. For that reason, researchers started 
to investigate the possibility for early detection, intervention and prevention; several lines of research emerged.

In the last two decades, substantial consideration was given to ultra-high risk (UHR) and clinical high-risk (CHR) states at the prodromal stage. These intervention strategies keep in line with the prototypic categorical concept of psychopathology ${ }^{24,25}$, aiming to intervene "transition" to psychosis disorder ${ }^{24}$. For categorization they rely heavily on the assessment of attenuated positive psychosis expression and a family history of psychotic symptoms ${ }^{26}$. To reduce false positives, these approaches prioritize specificity over sensitivity, accepting the possibility to miss potential vulnerable individuals ${ }^{26}$.

Initial findings suggested a high predictability of these concepts for clinically diagnoses. Forty percent of individuals categorized with UHR "transitioned" to psychosis disorder within a year ${ }^{27,28}$. However, later it became apparent that these estimates had been overestimated and contributed little to clinical utility. Estimates were magnified as the categories had typically been investigated in help-seeking individuals — with a greater risk to develop psychosis disorder - rather than in the general population ${ }^{29}$. The bias in sample enrichment was demonstrated by a study comparing 10-year transition rates from individuals presented at an early psychosis service for young people ${ }^{30}$. Individuals with a UHR diagnosis had practically a similar transition rate than individuals diagnosed with non-psychotic disorders; $17.3 \%$ and $14.6 \%$, respectively ${ }^{30}$. Furthermore, a recent cross-sectional study ${ }^{31}$, testing the UHR principles in the general population indicates that out of 2683 individuals, 15 fulfilled the attenuated psychotic symptom criteria. Out of these, only one also fulfilled the brief intermittent psychotic symptom criteria. Notably, non-psychotic disorders and functional deficit were common among individuals with any psychosis expression or cognitive impairments ${ }^{31}$.

Another critical point within this research line is the great emphasis on psychosis expression ${ }^{26}$. Indeed, as abovementioned, in the context of multidimensional psychopathology, psychosis expression may be a severity indicator for psychopathology ${ }^{13-20,32}$. However, in the general population, psychosis expression is not uncommon $(5-8 \%)^{33,34}$, and is transit for $80 \%$ of individuals ${ }^{33,35,36}$. The WHO World Mental Health Surveys report a temporal bidirectional association between common non-psychotic disorders and psychosis expression ${ }^{5}$. These findings question the utility of the high-risk approaches. As prospective research on the validity of the UHR and CHR concepts is missing, chapter 2 reports important findings on the longitudinal effect of these approaches on clinical psychosis in the general population. 
Another strain of research focuses on the above-mentioned multidimensional manifestation of the early stages of psychopathology. Research indicates that the initial expression of psychopathological phenotypes is marked by a mixture of symptom dimensions along with emotional and neurocognitive impairments including aberrant salience, motivational alterations, affective dysregulation, and anxiety states ${ }^{37}$. Symptom dimensions interact within and across traditional diagnosis categories in the clinical and general population ${ }^{19,20}$. Given its potential to detect mechanisms and processes along the psychosis spectrum, studying processes associated with nonclinical psychosis expression in the general population is an important methodological tool ${ }^{12}$. Therefore, the role of multidimensional psychopathology and subtle psychosis expression at population level is tested in chapter 3 .

To understand the differences between individuals developing PSD and those who do not, researchers try to apprehend whether population level psychosis expression and non-clinical phenotypes might occur through the same mechanism as in clinical samples. Different experimental tools and methodological paradigms can be considered of which some were investigated in this thesis. In chapter 4 an overview is given on studies conducted in the TwinssCan project, a Belgian prospective general population sample. Its main objective is to understand the contribution of genetic and environmental exposure on nonclinical dimensional phenotypes at a young age, before the onset of disorder, and their association with neurocognitive processes, such as salience attribution, sensitivity to social defeat, and reward sensitivity.

One of the studies conducted in the TwinssCan cohort is presented in chapter 5 . In that study, the white noise task, a promising tool to assess salient attribution, is applied to investigate whether the perception of speech illusions during nonsensical white noise is associated with trait expression of psychosis experiences (assessed with the Community Assessment of Psychic Experiences; CAPE and Structured Interview for SchizotypyRevised; SIS-R) in the general population. One promising theory to disentangle the processes associated with psychosis expression suggests that positive psychotic experiences might occur through the imbalance of top-down and bottom-up processes ${ }^{38}$. More specifically, individuals who experience psychosis expression may be more sensitive to attribute meaning to bottom-up signals. To illustrate the concept, imagine you are walking home during the day, top-down processes will not attribute much importance to common noises. However, if you walk home on a lonely street during the night, you might interpret noises as more threatening. While bottom-up signals may be the same for both scenarios, top-down processes give a different explanation for these signals. 
The dynamic nature of psychopathology is another important aspect of signs, symptoms and etiology within PSD that is evaluated in the TwinssCan project in chapter 6. Particular focus is drawn to the macro level (during months or years) changes of symptomatology, whereas studies indicate that psychopathology can best be understood when also looking at the micro level, symptom changes from moment-to-moment ${ }^{39}$. Therefore, studies using ecological momentary assessment - a tool to assess momentary variations in thoughts, symptoms, the context, and appraisal of that context — shed light on the fact that psychosis phenotypes dynamically manifest and change from moment-to-moment ${ }^{40,41}$.

Finally, an important line of research focuses its attention on the biological and environmental vulnerability to PSD. Extensive research shows that environmental factors such as childhood adversities and genomic liability are important determinants for the progression form subtle psychopathological expression towards clinical disorders ${ }^{37}$. As this is a major aspect of this thesis, an outline of gene-environment research in PSD is given in the following part.

\section{Environmental and genetic contribution to psychosis spectrum}

Understanding the etiopathology of PSD may improve diagnosis, prevention, and treatment. Therefore, huge efforts are made to apprehend the multifactorial etiology of PSD that includes a network of environmental and genetic liability ${ }^{42,43}$.

During the last decade the genetic field moved forward from single candidate gene studies to genome wide analyses, now explaining the $30-40 \%$ of the genetic variation of schizophrenia phenotype by single nucleotide polymorphisms (SNPs) and allowing for the calculation of a genetic risk score, the polygenic risk score for schizophrenia (PRS-SCZ) ${ }^{44}$. PRS-SCZ, a weighted sum of trait-associated alleles, substantially increases the prediction power to detect gene-environment interaction $(\mathrm{GxE})^{45}$ to gain insight into the complex pathoetiology of schizophrenia and related disorders.

Despite the substantial progress for molecular genetic analyses during the last years, the proportion of genetic liability that is explained by molecular genetic risk stays below that of heritability estimates from twin studies ${ }^{46}$, which suggest a heritability of $80 \%$. Therefore, researchers question whether at least some of the "missing heritability" is due to shared environmental exposure across individuals growing up in the same household. It is probable that genetic liability may sensitize individuals to environmental liability or drive them to more exposure ${ }^{42}$. Theories such as the liability-threshold model for PSD $^{47}$ introduce the idea that psychopathological expression is determined by aggregated genetic and environmental 
exposure. It predicts that individuals passing a critical threshold develop expression of psychosis phenotypes. Therefore, chapter 7 presents findings from the first systematic approach testing GxE using PRS-SCZ in a large international dataset with patients diagnosed with schizophrenia and healthy controls.

The pleiotropic nature of exposures is an important factor to understand gene-environmental contribution on the causal path from multidimensional psychopathology to PSD. Molecular genetic risk for schizophrenia is significantly shared with that of other psychopathological phenotypes ${ }^{48}$. A growing number of studies show that PRS-SCZ is pleiotropically associated with PSD, non-psychotic disorders as well as medical conditions ${ }^{49-51}$, subclinical multidimensional psychopathology ${ }^{51-54}$ and low quality of life $e^{55}$.

Akin to genetic liability, confirming the shared vulnerability theory, environmental exposure such as cannabis use and childhood adversity are similarly associated with psychosis, affective and stress-related phenotypes ${ }^{5-59}$. Accumulating evidence shows that genetic and environmental exposures are associated with more severe non-psychotic psychopathology which subsequently may non-casually be related to the experience of psychosis expression $^{15,32,60}$. Therefore, GxE on psychosis phenotypes in the general population is tested in chapters 6 and 9.

The role of environmental factors has long been recognized, and various exposures were identified to be associated with the risk to develop PSD. With varying effect size estimates, these environmental risk factors include cannabis use, childhood adversities (e.g., sexual abuse, emotional neglect), peer-bullying, urban environment, proxies of social exclusion (e.g., ethnic minority, immigration, and hearing impairment), season of birth, and obstetric and pregnancy complications ${ }^{61,62}$. Theories such as the diathesis model ${ }^{63}$ suggests that responses to current stress is substantially affected by sensitization from previous stress experiences, which is supported by epidemiological studies on the joint effect of childhood adversity and adulthood stressful life events ${ }^{57,64-68}$. Furthermore, accumulating studies find a dose-response relationship between exposure and psychopathological outcome: the more severe the exposure, the more severe the outcome $e^{32,58}$. However, while it is known that environmental factors are dependent on each other and correlate, knowledge is commonly based on hypothesis driven one-exposure on one outcome studies. Thus, although each exposure only presents a fraction of a dense network of non-genetic risk, studies on the exposome, akin the polygenic approach, stay behind that of the PRS-based research. In chapter 8 machine learning is applied to estimate a exposome score for schizophrenia, and in chapter 2 and 9 it is applied in the general population. Furthermore, in chapter 10 the independent and joint 
effect of exposomic and genomic liability for schizophrenia is tested across the psychosis spectrum in patients with schizophrenia, unaffected siblings and healthy comparisons.

\section{Thesis outline}

The current thesis aims to investigate and understand the development of PSD from subclinical to clinical psychosis phenotypes. More specifically, the first part of the thesis focuses on mechanisms associated with the expression of multidimensional nonclinical psychosis phenotypes. The second part of the thesis aims to close important gaps in the literature and push the scientific frontiers to assess an individual's entire exposomic and genomic liability. Therefore, a collection of significant findings on $\mathrm{GxE}$ and the exposome score for schizophrenia on pluripotent psychopathology in clinical and general population samples is presented.

In the first part of the thesis, testing the different approaches to conceptualize psychosis proneness in the general population, in chapter 2 , the predictive ability of CHR and nonpsychotic disorders on clinical psychosis is prospectively tested in a general population sample. Further, in chapter 3, the relationship between psychosis expression and amalgamated multidimensional psychopathology is investigated. To introduce the reader to the work in big data and several methodological tools to assess psychosis phenotypes in the general population, chapter 4 outlines the work conducted in the TwinssCan project and gives an overview of the important developments and studies on population level multidimensional psychopathology. This is followed by chapter 5 aiming to test whether subtle psychosis expression in the general population may be due to salient attribution and an imbalance between bottom-up and top-down processing; and thereby follows the same mechanisms as in clinical populations.

The second part of the thesis focuses on the independent and joint contribution of genetic and environmental liability to PSD. Chapter 6 tests whether polygenic liability for schizophrenia, childhood adversity and momentary stressors influences daily-life emotion dysregulation and psychosis proneness. Chapter 7 presents systematic analyses of $\mathrm{GxE}$ in a case-control sample with patients diagnosed with schizophrenia. In chapter 8, machine learning is applied to estimate an exposome score for schizophrenia. Following this, in a general population sample, chapter 9 tests whether underlying genomic and antecedent exposomic liability for schizophrenia sensitizes individuals' response to adulthood stressful life events on mental and physical health. Chapter 10 presents a comprehensive analyses of the interplay of exposomic and genomic liability across the psychosis spectrum in patients with schizophrenia, unaffected siblings and healthy comparisons. 


\section{References}

1. van Os J, et al. Evidence that polygenic risk for psychotic disorder is expressed in the domain of neurodevelopment, emotion regulation and attribution of salience. Psychol Med. 2017:1-16.

2. van Os J, et al. Replicated evidence that endophenotypic expression of schizophrenia polygenic risk is greater in healthy siblings of patients compared to controls, suggesting geneenvironment interaction. The EUGEI study. Psychol med. 2019;1-14.

3. Pazoki R, et al. Polygenic risk scores are associated with quality of life in schizophrenia.

4. Radhakrishnan R, et al. Gone to Pot - A Review of the Association between Cannabis and Psychosis. Front psychiatry. 2014:54

5. ten Have M, et al. Childhood maltreatment, vulnerability characteristics and adult incident common mental disorders: 3-year longitudinal data among $>10,000$ adults in the general population. $J$ Psychiatr Res. 2019:199-207.

6. Barzilay R, et al. Association between traumatic stress load, psychopathology, and cognition in the Philadelphia Neurodevelopmental Cohort. Psychol med. 2019:325-34.

7. Hailes H, et al. Long-term outcomes of childhood sexual abuse: an umbrella review. Lancet Psychiatry. 2019:830-39

8. van Nierop M, et al. Childhood trauma is associated with a specific admixture of affective, anxiety, and psychosis symptoms cutting across traditional diagnostic boundaries. Psychol med. 2015:1-11.

9. Radua J, et al. What causes psychosis? An umbrella review of risk and protective factors. World Psychiatry. 2018:49-66.

10. Belbasis L, et al. Risk factors and peripheral biomarkers for schizophrenia spectrum disorders: an umbrella review of meta-analyses. Acta Psychiat Scand. 2018:88-97.

11. Pruessner M, et al. The neural diathesis-stress model of schizophrenia revisited: An update on recent findings considering illness stage and neurobiological and methodological complexities. Neurosci biobehav rev. 2017:191-18.

12. McLaughlin K, et al. Childhood adversity, adult stressful life events, and risk of past-year psychiatric disorder: a test of the stress sensitization hypothesis in a population-based sample of adults. Psychol Med. 2010:1647-58.

13. Mansueto G, et al. Recent life events and psychosis: The role of childhood adversities. Psychiatry Res. 2017;1-7.

14. Holtzman C, et al. Stress and neurodevelopmental processes in the emergence of psychosis.

Neuroscience. 172-91.

15. Kendler K, et al. Childhood sexual abuse, stressful life events and risk for major depression in women. Psychol med. 2004:1-7.

16. Morgan C, et al. Modelling the interplay between childhood and adult adversity in pathways to psychosis: initial evidence from the AESOP study Psychol Med. 2014:1-12.

17. Ayesa-Arriola R, et al. The synergetic effect of childhood trauma and recent stressful events in psychosis: associated neurocognitive dysfunction. Acta Psychiatr Scand. 2019:1-9.

18. Lataster J, et al. Adversity and psychosis: a 10-year prospective study investigating synergism between early and recent adversity in psychosis. Acta Psychiat Scand. 2012:1-11.

19. Beards S, et al. Life events and psychosis: a review and meta-analysis. Schizophr Bull. 2013:1-7.

20. Suliman $S$, et al. Cumulative effect of multiple trauma on symptoms of posttraumatic stress disorder, anxiety, and depression in adolescents. Compr Psychiatry. 2009:121-27.

21. Evans G, et al. Cumulative risk and child development. Psychol Bull. 2013;1342-96.

22. Karam E, et al. Cumulative traumas and risk thresholds: 12-month PTSD in the World Mental Health surveys. Depress Anxiety. 2014:1-12

23. Srihari $\mathrm{V}$, et al. Is early intervention for psychosis feasible and effective? Psychiatr Clin. 2012:1-18.

24. Van Os J, et al. Toward a world consensus on prevention of schizophrenia. Dialogues Clin Neurosci. 2005:53-67.

25. Fusar-Poli P, et al. Lessons learned from the psychosis high-risk state: towards a general staging model of prodromal intervention. Psychol Med. 2014:1-7.

26. Yung A, et al The ultra high risk approach to define psychosis risk. Curr pharm des. 2012:346-50.

27. Yung A, et al. Psychosis prediction: 12-month follow up of a high-risk ("prodromal") group. Schizophr Res. 2003:21-32.

28. Yung A, et al. Risk factors for psychosis in an ultra high-risk group: psychopathology and clinical features. Schizophr Res. 2004:131-142.

29. van Os J, et al. A critique of the "ultra-high risk" and "transition" paradigm. World Psychiatry. 2017:1-6

30. Conrad A, et al. Utility of risk-status for predicting psychosis and related outcomes: evaluation of a 10year cohort of presenters to a specialised early psychosis community mental health service. Psychiatry Res. 2017:336.

31. Schultze-Lutter F, et al. Prevalence and clinical relevance of interview-assessed psychosis-risk symptoms in the young adult community. Psychol med. 2018:1-11.

32. Guloksuz S, et al. Evidence that the presence of psychosis in non-psychotic disorder is environmentdependent and mediated by severity of nonpsychotic psychopathology. Psychol med. 2015.

33. Linscott $\mathrm{R}$, et al. An updated and conservative systematic review and meta-analysis of epidemiological evidence on psychotic experiences in children and adults: on the pathway from proneness to persistence to dimensional expression across mental disorders. Psychol Med. 2013:1-16. 
34. Van Os J, et al. A systematic review and metaanalysis of the psychosis continuum: evidence for a psychosis proneness-persistence-impairment model of psychotic disorder. Psychol med. 2009:1-16.

35. Hanssen $M$, et al. The incidence and outcome of subclinical psychotic experiences in the general population. Br J Clin Psychol. 2005:181-91.

36. Cougnard A, et al. Does normal developmental expression of psychosis combine with environmental risk to cause persistence of psychosis? A psychosis proneness-persistence model. Psychol med. 2007.

37. van Os J. The dynamics of subthreshold psychopathology: implications for diagnosis and treatment. Am j psychiatry. 2013:1-3.

38. Aleman A, et al. Cognitive basis of hallucinations in schizophrenia: role of top-down information processing. Schizophr Res. 2003:175-185.

39. Nelson B, et al. Moving from static to dynamic models of the onset of mental disorder: a review. JAMA psychiatry. 2017:528-34.

40. Myin-Germeys I, et al. Stress-reactivity in psychosis: evidence for an affective pathway to psychosis. Clinical psychology review. 2007:1-15.

41. Oorschot M, Kwapil T, Delespaul P, et al. Momentary assessment research in psychosis. Psychol Assess. 2009:1-7.

42. van Os J, et al. The environment and schizophrenia. Nature. 2010:203-12.

43. Van Os J, et al Gene-environment interactions in schizophrenia: review of epidemiological findings and future directions. Schizophr Bull. 2008:1-16.

44. Ripke $S$, et al. Biological insights from 108 schizophrenia-associated genetic loci. Nat neurosci. 2014:421-27.

45. Iyegbe $\mathrm{C}$, et al. The emerging molecular architecture of schizophrenia, polygenic risk scores and the clinical implications for $\mathrm{GxE}$ research. Soc psychiatry psychiatr epidemiol. 2014:169-182.

46. Hilker R, et al. Heritability of Schizophrenia and Schizophrenia Spectrum Based on the Nationwide Danish Twin Register. Biol Psychiatry. 2018:1-6.

47. Gottesman, I, et al. A polygenic theory of schizophrenia. Int J ment health. 1967:199-205.

48. The Brainstorm Consortium, et al. Analysis of shared heritability in common disorders of the brain. Science. 2018:eaap8757.

49. Zheutlin A, et al. Penetrance and Pleiotropy of Polygenic Risk Scores for Schizophrenia in 106,160 Patients Across Four Health Care Systems. Am j psychiatry. 2019:846-55.

50. Richardson T, et al. An atlas of polygenic risk score associations to highlight putative causal relationships across the human phenome. Elife. 2019.

51. Mistry S, et al. The use of polygenic risk scores to identify phenotypes associated with genetic risk of schizophrenia: systematic review. Schizophr res. 2018:2-8

52. Jones H, et al. Phenotypic Manifestation of Genetic Risk for Schizophrenia During Adolescence in the General Population. JAMA psychiatry. 2016:1-7.
53. van Os J, et al. Evidence that polygenic risk for psychotic disorder is expressed in the domain of neurodevelopment, emotion regulation and attribution of salience. Psychol Med. 2017:1-16

54. van Os J, et al. Replicated evidence that endophenotypic expression of schizophrenia polygenic risk is greater in healthy siblings of patients compared to controls, suggesting geneenvironment interaction. The EUGEI study. Psychol med. 2019:1-14.

55. Pazoki R, et al. Polygenic risk scores are associated with quality of life in schizophrenia. submitted.

56. Radhakrishnan R, et al. Gone to Pot - A Review of the Association between Cannabis and Psychosis. Front psychiatry. 2014:54.

57. ten Have M, et al. Childhood maltreatment, vulnerability characteristics and adult incident common mental disorders: 3 -year longitudinal data among $>10,000$ adults in the general population. $J$ psychiatr res. 2019;199-207.

58. Barzilay R, et al. Association between traumatic stress load, psychopathology, and cognition in the Philadelphia Neurodevelopmental Cohort. Psychol med. 2019:325-34.

59. Hailes $\mathrm{H}$, et al. Long-term outcomes of childhood sexual abuse: an umbrella review. Lancet Psychiatry. 2019:830-39.

60. van Nierop M, et al. Childhood trauma is associated with a specific admixture of affective, anxiety, and psychosis symptoms cutting across traditional diagnostic boundaries. Psychol med. 2015:1277-88.

61. Radua J, et al. What causes psychosis? An umbrella review of risk and protective factors. World Psychiatry. 2018:49-66.

62. Belbasis L, et al. Risk factors and peripheral biomarkers for schizophrenia spectrum disorders: an umbrella review of meta-analyses. Acta Psychiat Scand. 2018:88-97.

63. Pruessner M, et al. The neural diathesis-stress model of schizophrenia revisited: An update on recent findings considering illness stage and neurobiological and methodological complexities. Neurosci Biobehav Rev. 2017:191-218.

64. McLaughlin K, et al. Childhood adversity, adult stressful life events, and risk of past-year psychiatric disorder: a test of the stress sensitization hypothesis in a population-based sample of adults. Psychol Med. 2010:1647-58.

65. Mansueto G, et al. Recent life events and psychosis: The role of childhood adversities. Psychiatry Res. 2017:1-16.

66. Holtzman C, et al. Stress and neurodevelopmental processes in the emergence of psychosis. Neuroscience. 2013:172-91.

67. Kendler K, et al. Childhood sexual abuse, stressful life events and risk for major depression in women. Psychol med. 2004:1475-1482.

68. Morgan C, et al. Modelling the interplay between childhood and adult adversity in pathways to psychosis: initial evidence from the AESOP study. Psychol Med. 2014:407-19. 





\section{ASSOCIATION OF PRECEDING PSYCHOSIS RISK STATES AND NON-PSYCHOTIC MENTAL DISORDERS WITH INCIDENCE OF CLINICAL PSYCHOSIS IN THE GENERAL POPULATION: A PROSPECTIVE STUDY IN THE NEMESIS-2 COHORT}

Sinan Guloksuz*, Lotta-Katrin Pries*, Margreet ten Have,

Ron de Graaf, Saskia van Dorsselaer, Boris Klingenberg, Maarten Bak, Bochao D. Lin, Kristel R. van Eijk, Philippe Delespaul, Therese van Amelsvoort, Jurjen J. Luykx, Bart P. F. Rutten, Jim van Os 


\section{Abstract}

The validity and clinical utility of the concept of "clinical high risk" (CHR) for psychosis have so far been investigated only in risk-enriched samples in clinical settings. In this population-based prospective study, we aimed - for the first time - to assess the incidence rate of clinical psychosis and estimate the population attributable fraction (PAF) of that incidence for preceding psychosis risk states and DSM-IV diagnoses of non-psychotic mental disorders (mood disorders, anxiety disorders, alcohol use disorders, and drug use disorders). All analyses were adjusted for age, gender and education. The incidence rate of clinical psychosis was 63.0 per 100,000 person-years. The mutually-adjusted Cox proportional hazards model indicated that preceding diagnoses of mood disorders (hazard ratio, $\mathrm{HR}=10.67,95 \% \mathrm{CI}: 3.12-36.49)$, psychosis high-risk state (HR=7.86, 95\% CI: 2.76-22.42) and drug use disorders ( $\mathrm{HR}=5.33,95 \% \mathrm{CI}$ : 1.61-17.64) were associated with an increased risk for clinical psychosis incidence. Of the clinical psychosis incidence in the population, 85.5\% (95\% CI: 64.6-94.1) was attributable to prior psychopathology, with mood disorders $(\mathrm{PAF}=66.2,95 \% \mathrm{CI}: 33.4-82.9)$, psychosis high-risk state $(\mathrm{PAF}=36.9,95 \% \mathrm{CI}: 11.3-$ 55.1), and drug use disorders ( $\mathrm{PAF}=18.7,95 \% \mathrm{CI}:-0.9$ to 34.6) as the most important factors. Although the psychosis high-risk state displayed a high relative risk for clinical psychosis outcome even after adjusting for other psychopathology, the PAF was comparatively low, given the low prevalence of psychosis high-risk states in the population. These findings provide empirical evidence for the "prevention paradox" of targeted CHR early intervention. A comprehensive prevention strategy with a focus on broader psychopathology may be more effective than the current psychosis-focused approach for achieving population-based improvements in prevention of psychotic disorders.

Key words: Psychosis, ultra-high risk, clinical high risk, mood disorders, drug use disorders, early intervention, prevention, at risk mental states 
Early intervention in psychosis has been an active area of investigation in the mental health field over the past quarter century. Compelling evidence indicates that specialized early intervention services for first-episode psychosis yield better short-term clinical outcomes in all measurable domains compared to usual treatment ${ }^{1}$. In addition, it has been suggested that shortening the duration of untreated psychosis leads to a better prognosis over the course of the illness ${ }^{2}$. The field has thus moved forward with the idea of intervening even earlier by detecting psychosis at the preclinical phase of "ultra-high risk" (UHR), also known as "clinical high risk" (CHR).

Over the last decade, the validity and clinical utility of the CHR paradigm have been widely investigated in help-seeking participants sampled in clinical settings (risk-enriched samples) ${ }^{3}$. The CHR paradigm relies on the frequency and severity of positive psychotic symptoms to identify the at-risk state and determine the risk of transition to psychosis ${ }^{3}$.

Early studies reported up to $40 \%$ transition rates in CHR samples, but these rates consistently decreased as data accumulated over time, with recent meta-analytical estimates showing less than half of the initially reported rates: $15 \%$ over a mean period of 38 months $s^{4}$, or $4.7 \%$ per year. This sizeable reduction in the transition rates may be due to a dilution effect, which is the by-product of the increased awareness of subtle psychotic states and broader outreach of early intervention services, leading to an increase in self-referrals, and thereby inflating false positives in more recent CHR samples.

Following our critical perspective papers on the CHR concept ${ }^{3,5}$, an intense debate has started, splitting the field into proponents ${ }^{6-8}$, opponents ${ }^{9-12}$, and those with ambivalent attitudes toward the CHR concept ${ }^{13-16}$.

In parallel with the growing interest in understanding early stages of psychopathology for early detection and intervention in clinical settings, the psychosis phenotype has been widely studied in general population datasets.

These population-based epidemiological studies have revealed two important findings. First, subtle positive psychotic experiences (PEs) are not as rare as once assumed, with prevalence rates varying between 5 and $8 \%{ }^{17}$. Second, PEs are temporally associated with help-seeking ${ }^{18}$, suicidal behavior ${ }^{19,20}$, poor functioning ${ }^{21,22}$, decline in cognitive capacity ${ }^{23}$, affective dysregulation, and a multitude of mental disorders, including but not limited to psychosis spectrum disorder ${ }^{24-26}$. In that sense, PEs in the general population appear to be clinically valuable as a severity marker, but they do not imply diagnostic specificity. 
With the exception of the cross-sectional Bern Epidemiological At-Risk (BEAR) study, these two lines of research - clinical and population-based - have yet to be crossed. Particularly relevant is the issue of help-seeking behavior of individuals, which is included in the CHR concept but not in the population studies of PEs. The BEAR study demonstrated that the $\mathrm{CHR}$ is not a frequent but a clinically relevant state, which is associated with increased odds for present mental disorder diagnosis and impaired functioning ${ }^{27}$. Further, the CHR entity shares the same etiological factors with PEs in community studies and psychotic disorders in the clinical samples, providing support for the notion of etiological continuity across the psychosis spectrum.

Although the findings from the cross-sectional BEAR study may provide some insight into the characteristics of the CHR state in an epidemiologically representative sample, the core issue of progression of psychosis in the framework of the CHR-transition paradigm has not been longitudinally tested to date in an unbiased general population cohort.

In this study, we aimed to explore the notions of "risk" and "transition" in the general population, for the first time, by estimating the population attributable fraction (PAF) of clinical psychosis incidence (the proportion of clinical psychosis outcome that would have been avoided, had the risk factors been eliminated) for the preceding psychosis risk states and DSM-IV diagnoses of non-psychotic mental disorders.

\section{Methods}

\section{Study cohort}

The Netherlands Mental Health Survey and Incidence Study-2 (NEMESIS-2) was designed to investigate the prevalence, incidence, course and consequences of mental disorders in the Dutch general population. The study was approved by the Medical Ethics Review Committee for Institutions on Mental Health Care, and written informed consent was collected from participants at each wave ${ }^{28,29}$. A multistage random sampling procedure was applied to ensure sample representativeness in regard to age (between 18 and 65 years), region, as well as population density. Participants were excluded if they were not proficient in Dutch.

The NEMESIS-2 cohort includes four waves. The baseline data (T0) were assessed from 2007 to 2009, and were followed up at year 3 (T1), year 6 (T2) and year 9 (T3). The first wave (T0) enrolled 6,646 participants (response rate 65.1\%; average interview duration: 95 $\mathrm{min})$. Response rates at $\mathrm{T} 1, \mathrm{~T} 2$ and $\mathrm{T} 3$ were $80.4 \%(\mathrm{~N}=5,303$; interview duration: $84 \mathrm{~min})$, 
87.8\% ( $\mathrm{N}=4,618$; interview duration: $83 \mathrm{~min})$, and $86.8 \%(\mathrm{~N}=4,007$; interview duration: $102 \mathrm{~min})$, respectively ${ }^{30}$.

Non-clinician, trained interviewers applied the Composite International Diagnostic Interview (CIDI) version 3.0 $0^{31,32}$ and additional questionnaires during home visits. Rates at baseline reflect lifetime occurrence; rates at T1, T2 and T3 reflect 3-year interval occurrence. Attrition between T0 and T3 was not significantly associated with any of the individual 12month mental disorders at T0 after controlling for socio-demographic characteristics ${ }^{33}$.

\section{Psychosis risk strata}

In accordance with the clinical high-risk framework ${ }^{3}$ and previous analyses conducted in the NEMESIS-2 cohort $^{34,35}$, psychosis risk strata were defined based on the degree of positive psychotic symptomatology, help-seeking attempt, antipsychotic treatment, and service use and admission for psychotic symptomatology.

At each time point, positive psychotic symptoms were assessed using a 20-item binaryresponse questionnaire that is based on CIDI 1.1 and specifically developed for evaluating psychotic symptoms ${ }^{36,37}$, since previous studies have demonstrated that earlier CIDI versions were not adequately capturing positive psychotic symptomatology. Positive reports (positive response to at least one item) were reassessed and validated over a clinical telephone interview conducted by trained graduate psychologists and discussed with a clinical experienced psychiatrist ${ }^{38}$, and participants were asked whether they had sought help for these symptoms. At each time point, antipsychotic prescription, service use and admission were explored using an adaptation of the self-constructed NEMESIS-1 questionnaire ${ }^{39}$.

Psychosis risk strata consisted of the following non-overlapping categories: reference group (no psychosis expression), low-risk (endorsement of a single positive psychotic item that did not require help-seeking or treatment), moderate-risk (endorsement of multiple positive psychotic items that did not require help-seeking or treatment), high-risk (endorsement of at least one positive psychotic item that required help-seeking but not antipsychotic treatment or admission), and clinical psychosis (endorsement of at least one positive psychotic item that required help-seeking and antipsychotic treatment or admission to a health care service). The primary outcome of the study was the category of clinical psychosis. The low-risk, moderaterisk, and high-risk strata served as risk states. 


\section{Preceding diagnosis of DSM-IV mental disorders}

The CIDI $3.0^{31}$ was used to assess the following four domains of DSM-IV mental disorders at each follow-up visit (diagnosis over the last 3-year period, such that T1 assessment covers the period between $\mathrm{T} 0$ and T1; T2 assessment covers the period from T1 to T2, and so on): mood disorders (major depressive disorder, bipolar disorder, dysthymia); anxiety disorders (social phobia, specific phobia, panic disorder, generalized anxiety disorder, agoraphobia without panic disorder); alcohol use disorders (alcohol abuse and dependence); and drug use disorders (drug abuse and dependence).

\section{Statistical analyses}

Analyses were conducted using Stata version 16.0. Participants diagnosed with psychotic disorders $(\mathrm{N}=43,0.7 \%)$ or bipolar disorder $\mathrm{I}(\mathrm{N}=73,1.1 \%)$ at baseline were excluded from the analysis. A priori defined psychosis risk strata were validated by using cumulative measures of environmental and genetic liability to schizophrenia.

Adopting our previously validated estimates for constructing cumulative environmental load in a Dutch cohort (GROUP) ${ }^{40}$, we generated the exposome score for schizophrenia (ESSCZ) by summing log odds weighted environmental exposures, including cannabis use, hearing impairment, winter birth, and five childhood adversity domains (sexual, physical and psychological abuse, emotional neglect and bullying). Analyses were carried out using the dichotomous environmental risk state: the highest quartile, ES-SCZ $>75 \%$, was considered the binary environmental vulnerability for schizophrenia, guided by the definition in our previous study (hereafter: $\left.\mathrm{ES}-\mathrm{SCZ}_{75}\right)^{41}$.

The validation of the psychosis risk strata using polygenic risk score for schizophrenia (PRSSCZ) was performed in the genotyped sample $(\mathrm{N}=3,104)$. Analyses were carried out using the molecular genetic risk state, guided by the definition in our previous study ${ }^{41}$ : the highest quartile of PRS-SCZ $>75 \%$ was considered the binary genetic liability for schizophrenia (hereafter: PRS-SCZ ${ }_{75}$ ).

Multinomial logistic regression models using the MLOGIT command were performed to analyze the association of psychosis risk strata ("no-risk" group as the reference) with ES$\mathrm{SCZ}_{75}$ and PRS-SCZ 75 , respectively. Consistent with our previous work in NEMESIS-2, the validation analysis of the strata included observations from all assessment points, that were analyzed multi-cross-sectionally in the "long format" (each participant contributing four observations: T0, T1, T2 and T3). To correct for the clustering of multiple observations 
within participants, the CLUSTER option was used to estimate cluster-robust standard errors (SEs).

The relative risk ratios (RRRs) at each psychosis risk stratum for ES-SCZ 75 and PRS-SCZ 75 were compared using the Wald test. All analyses were adjusted for gender, age (continuous), and four-level education (1- primary school, 2- lower secondary education, 3- higher secondary education, 4- higher professional education). Analyses of PRS-SCZ 75 were additionally corrected for population stratification adjusted using the first three principal components.

The crude incidence rates with $95 \%$ CIs of each psychosis risk stratum per 100,000 personyears were estimated in participants with at least one follow-up interview. Two-sided exact significance tests were applied to compare incidence rates over and below 35 years of age at the study entry.

The Cox proportional hazards models, with the time-on-study as the time scale over the whole study period from T0 to T3, were used to estimate the adjusted (age, gender and education) and multivariable adjusted hazard ratios (HRs) and 95\% CIs for the associations of clinical psychosis outcome with the time-varying factors of preceding psychosis risk states and diagnoses of anxiety, mood, alcohol use, and drug use disorders, respectively.

Efron's method was used for handling ties ${ }^{42}$. To take into account clustering of multiple observations within participants, a robust Hubert/White sandwich estimator was applied ${ }^{43}$. The proportional-hazards assumptions were confirmed using the Schoenfeld residuals and $-\ln (-\ln [$ survival $])$ plots, also adjusted for covariates ${ }^{44}$. Potential bias due to unmeasured confounders was assessed using the E-value, which is the minimum strength of association that an unmeasured confounder must have with both the exposure and the outcome to negate the observed association ${ }^{45}$.

By using the PUNAFCC command ${ }^{46}$ with the UNCONDITIONAL option that accounts for the sampling variability of the covariates, the attributable fraction and the PAF with $95 \%$ CIs for each risk factor were estimated. Under the assumption that the different risk groups are causally associated with the clinical psychosis outcome, the PAF shows the proportion of clinical psychosis disease burden that might be prevented if the risk were eliminated ${ }^{47}$. The nominal significance threshold was set two-sided at $\mathrm{p}=0.05$. 


\section{Results}

Table 1 shows the demographic features and the frequency of preceding psychosis risk states and DSM-IV diagnoses of non-psychotic mental disorders (as assessed at T0) in participants with at least one followup interview $(\mathrm{N}=5,303)$.

Table 2 reports the validation of the psychosis risk strata by using the ES$\mathrm{SCZ}_{75}$ and PRS-SCZ 75 . In comparison to the reference group, ES-SCZ 75 and PRS-SCZ 75 showed a progressively greater magnitude of association with increasing psychosis risk strata, with RRRs ranging between 1.44 and 3.49 for the ES-SCZ 75 , and between 0.85 and 3.63 for the PRS-SCZ 75 .

Table 2 Validation of the psychosis risk strata

\begin{tabular}{|c|c|c|c|c|c|c|c|c|c|}
\hline & \multicolumn{3}{|c|}{$\begin{array}{c}\text { Reference group } \\
\text { ("no-risk") }\end{array}$} & \multicolumn{2}{|c|}{ Low-risk } & \multicolumn{2}{|c|}{ Moderate-risk } & \multicolumn{2}{|c|}{ High-risk } \\
\hline & & & & Wald & & Wald & & Wald & \\
\hline & RRR & $95 \% \mathrm{CI}$ & $\mathrm{p}$ & $\chi^{2}$ & $\mathrm{p}$ & $\chi^{2}$ & $\mathrm{p}$ & $\chi^{2}$ & $\mathrm{p}$ \\
\hline \multicolumn{10}{|l|}{$E S-S C Z_{75^{2}}$} \\
\hline Low-risk & 1.44 & $1.22-1.69$ & $<0.001$ & & & - & & & - \\
\hline Moderate-risk & 2.06 & $1.63-2.61$ & $<0.001$ & 7.40 & 0.007 & - & & & - \\
\hline High-risk & 2.72 & $2.17-3.41$ & $<0.001$ & 23.15 & $<0.001$ & 3.26 & 0.071 & - & - \\
\hline Clinical psychosis & 3.49 & $1.80-6.79$ & $<0.001$ & 6.52 & 0.011 & 2.17 & 0.141 & 0.53 & 0.469 \\
\hline \multicolumn{10}{|l|}{$P R S-S C Z_{75^{a, b}}$} \\
\hline Low-risk & 0.85 & $0.66-1.10$ & 0.217 & - & - & - & - & - & - \\
\hline Moderate-risk & 1.25 & $0.88-1.79$ & 0.215 & 3.77 & 0.052 & - & - & - & - \\
\hline High-risk & 1.55 & $1.11-2.16$ & 0.010 & 9.07 & 0.003 & 0.87 & 0.350 & - & - \\
\hline Clinical psychosis & 3.63 & $1.23-10.71$ & 0.020 & 6.62 & 0.010 & 3.43 & 0.064 & 2.33 & 0.127 \\
\hline
\end{tabular}

RRR - relative risk ratio, ES-SCZ 75 - exposome score for schizophrenia (75\% cut-point), PRS-SCZ 75 - polygenic risk score for schizophrenia (75\% cut-point)

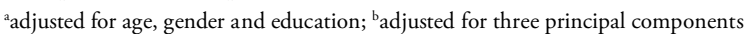

The ES-SCZ 75 was significantly associated with the low-risk, moderate-risk, high-risk, and clinical psychosis strata. The PRS-SCZ 75 was significantly associated with the high-risk and clinical psychosis strata, which were therefore validated. Additional post-hoc group comparisons of the ES-SCZ 75 across strata showed significant differences in low-risk vs. 
moderate-risk, low-risk vs. high-risk, and low-risk vs. clinical psychosis; while analysis of the $\mathrm{PRS}_{-} \mathrm{SCZ}_{75}$ across strata showed significant differences in low-risk vs. high-risk, and low-risk vs. clinical psychosis.

The incidence rate of clinical psychosis was 63.0 per 100,000 person-years (95\% CI: 42.992.6), with comparable rates for individuals under 35 years $(50.1$ per 100,000 person-years, 95\% CI: 20.9-120.5) and 35 years of age and above (67.1 per 100,000 person-years, $95 \%$ CI: 43.8-103.0; incidence rate ratio $=1.34,95 \% \mathrm{CI}: 0.49-4.55, p=0.58)$.

Figures 1 and 2 show the HRs for psychosis risk categories and diagnoses of non-psychotic mental disorders. Preceding diagnoses of mood, drug use, and anxiety disorders, along with psychosis high-risk state, showed an increased risk for clinical psychosis incidence in the age, gender and education-adjusted model. In the multivariable adjusted model, preceding diagnoses of $\operatorname{mood}(\mathrm{HR}=10.67,95 \% \mathrm{CI}$ : 3.12-36.49), psychosis high-risk state $(\mathrm{HR}=7.86$, 95\% CI: 2.76-22.42) and drug use disorders ( $\mathrm{HR}=5.33,95 \% \mathrm{CI}: 1.61-17.64)$ were associated with an increased risk for clinical psychosis incidence.

Figure 1 Hazard ratios $(95 \% \mathrm{Cl})$ for clinical psychosis incidence in the age, gender and educationadjusted model

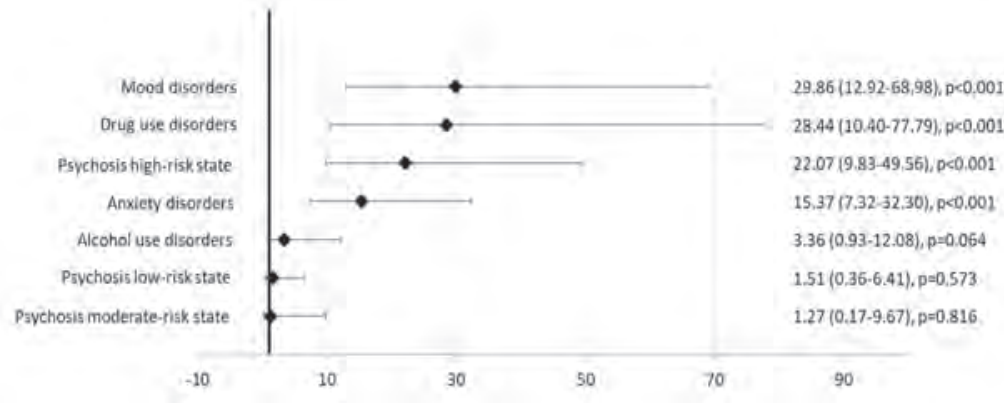

Figure 2 Hazard ratios $(95 \% \mathrm{Cl})$ for clinical psychosis incidence in the multivariable adjusted model

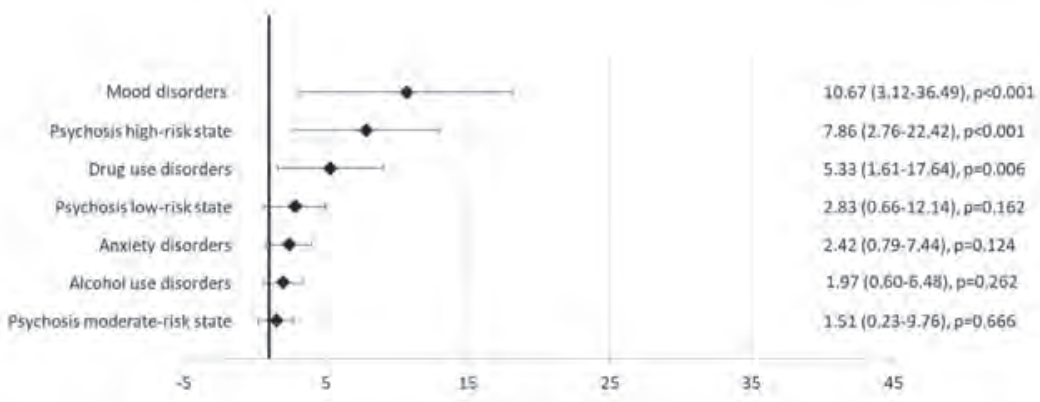


The E-values for the association of incident clinical psychosis with preceding diagnoses and risk states were 20.8 for mood disorders, 15.2 for psychosis high-risk state, 10.1 for drug use disorders, 5.1 for psychosis low-risk state, 4.3 for anxiety disorders, 3.4 for alcohol use disorders, and 2.4 for psychosis moderate-risk state.

Figures 3 and 4 show the PAFs for psychosis risk categories and diagnoses of non-psychotic mental disorders. The estimation of the PAFs in the multivariable adjusted model indicated that $85.5 \%$ (95\% CI: 64.6-94.1) of the clinical psychosis incidence could have been avoided if all psychosis risk states and non-psychotic mental disorders had been prevented. The most important factors were mood disorders (PAF $=66.2$, 95\% CI: 33.4-82.9), psychosis highrisk state $(\mathrm{PAF}=36.9,95 \% \mathrm{CI}: 11.3-55.1)$, and drug use disorders $(\mathrm{PAF}=18.7,95 \% \mathrm{CI}$ : 0.9 to 34.6$)$.

Figure 3 Population attributable fractions $(95 \% \mathrm{Cl})$ for clinical psychosis incidence in the age, gender and education-adjusted model

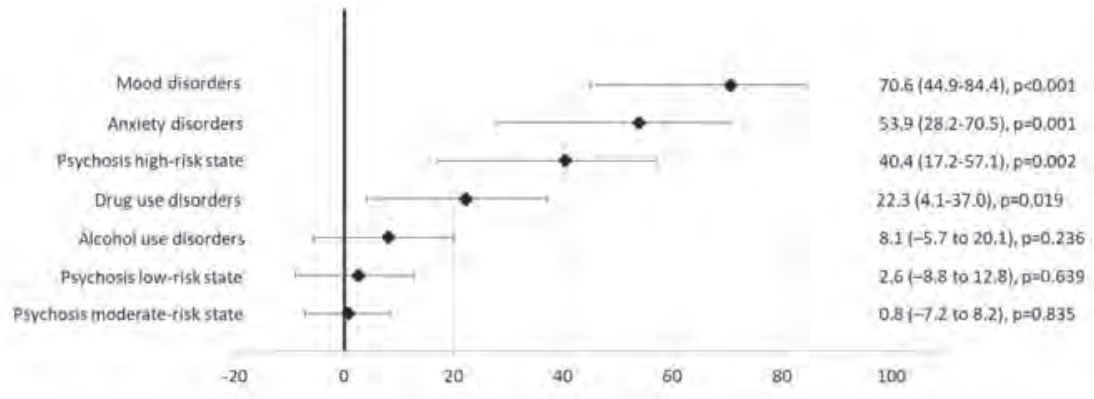

Figure 4 Population attributable fractions $(95 \% \mathrm{Cl})$ for clinical psychosis incidence in the multivariable adjusted model

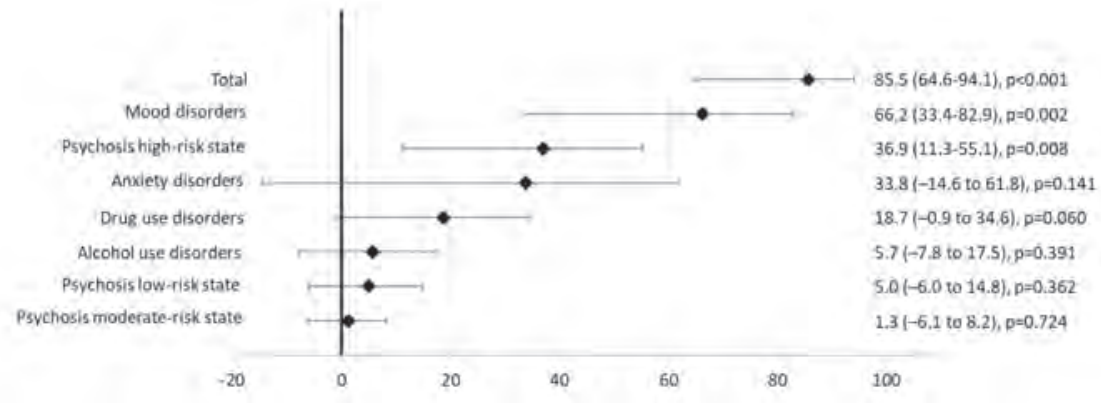


Further, we estimated the PAF for the subpopulation of the psychosis high-risk state. This restricted analysis revealed that $87.3 \%$ (95\% CI: 63.7-95.5) of the clinical psychosis incidence could have been avoided if the psychosis high-risk state had been prevented when other psychopathology remained the same; while the combined PAF for non-psychotic DSM diagnoses was 71.8\% (95\% CI: 33.6-88.0) when all other factors remained as observed.

\section{Discussion}

The main findings of this first population-based study of longitudinal risk for clinical psychosis as a function of the preceding psychosis risk states and DSM-IV diagnoses of nonpsychotic mental disorders were as follows: a) prior psychopathology accounted for a total of $85.5 \%$ of the incidence of clinical psychosis outcome in the multivariable analysis, with mood disorders, psychosis high-risk state, and drug use disorders independently contributing to clinical psychosis risk; b) the significant reduction of mutually-adjusted HRs in the multivariable model put the importance of comorbidity in perspective. These findings have important public health implications for early intervention strategies.

The PAFs for each psychopathology measure estimated in the final model were considerably lower than those estimated in the individual models, which were adjusted only for age, gender and education. The substantial differences in estimates between models demonstrate the importance of accounting for comorbidity beyond isolated measures of psychosis risk to yield more accurate PAF estimates for mental disorders.

We observed relatively large PAFs, except those for psychosis low-risk state, psychosis moderate-risk state, and alcohol use disorders, which were negligible. Preceding diagnosis of mood disorders was strongly associated with clinical psychosis outcome, and by far had the largest PAF, followed by psychosis high-risk state, anxiety disorders, and drug use disorders. In addition to the marked reduction of PAF estimates in the final model, PAF for anxiety disorders, although still noteworthy, was not statistically significant anymore.

From a public health perspective, a 10 -fold increase in risk for clinical psychosis incidence attributable to mood disorders highlights the importance of addressing the prevention of these disorders to reduce the burden of psychosis in the general population.

Given the fact that non-psychotic disorders are highly prevalent among individuals with CHR and likely to influence the longitudinal outcomes ${ }^{48-50}$, we estimated the risk attributable to these disorders in the subpopulation of participants with psychosis high-risk state. The joint PAF for all non-psychotic mental disorders was noteworthy but still lower than the 
individual PAF for psychosis high-risk state when everything else remained the same in this subpopulation.

Even though the psychosis high-risk state group displayed a high relative risk for clinical psychosis outcome even after adjusting for other psychopathology, the PAF was comparatively low. In contrast, anxiety disorders had a high PAF with respect to HR. This discrepancy between PAF and HR can be understood by examining the estimation method of PAF, which accounts for the prevalence of the risk factor in the population in addition to the strength of the association between outcome and risk factor.

In this regard, addressing the psychosis high-risk state in a sample enriched for clinical psychosis risk may appear to be an effective strategy at first glance. However, an early intervention strategy targeting high-risk state only will have minimal impact on reducing the population burden of psychotic disorders, because of the low prevalence of that state in the general population ${ }^{27}$. Further, efforts to case-finding will require major resources, given the rarity of psychosis high-risk state in the population. These findings provide empirical evidence for the "prevention paradox" and echo our concerns over the effectiveness and the economic feasibility of targeted CHR early intervention programs at the population level ${ }^{3,5}$.

In this first study investigating the PAFs of psychopathology categories for clinical psychosis in the general population, we used multivariable modeling to yield more accurate estimates ${ }^{51}$. The large and representative population cohort collected at four time-points over 9 years was a major strength. The clinical psychosis outcome incidence and the point prevalence of psychosis high-risk state were comparable to the population estimates in the literature ${ }^{27,52}$, thereby providing further support for the validity of our psychosis risk stratification approach in this population, that was guided by our previous work and verified using cumulative measures of environmental and genetic liability to schizophrenia. Nevertheless, future studies could benefit from a detailed clinical assessment and multi-source data including electronic health records to minimize measurement bias. Finally, the high E-values (20.8 for mood disorders, 15.2 for psychosis high-risk state, 10.1 for drug use disorders) show that unmeasured confounding is unlikely to influence the current significant findings. Notwithstanding, strong causal inferences should be avoided, considering the observational nature of the study.

Our results provide initial empirical evidence that a comprehensive prevention strategy with a focus on broader measures of psychopathology may be more effective than the current psychosis-focused approach in achieving population-based improvements for prevention of 
psychotic disorders. Guided by a public health approach, a fully-integrated universal mental health care system that ensures low-threshold entry and rapid access may serve as a more efficient strategy for improving population-based estimates of mental health including psychosis prevention, and may counter the trend of balkanizing mental health care to smaller and competing units ${ }^{53}$. 


\section{References}

1. Correll CU, Galling B, Pawar A et al. Comparison of early intervention services vs treatment as usual for early-phase psychosis: a systematic review, metaanalysis, and meta-regression. JAMA Psychiatry 2018;75:555-65.

2. Srihari VH, Shah J, Keshavan MS. Is early intervention for psychosis feasible and effective? Psychiatr Clin North Am 2012;35:613-31.

3. van Os J, Guloksuz S. A critique of the "ultra-high risk" and "transition" paradigm. World Psychiatry 2017;16:200-6.

4. Fusar-Poli P, Schultze-Lutter F, Cappucciati M et al. The dark side of the moon: meta-analytical impact of recruitment strategies on risk enrichment in the clinical high risk state for psychosis. Schizophr Bull 2015;42:732-43.

5. Guloksuz S, van Os J. Need for evidence-based early intervention programmes: a public health perspective. Evid Based Ment Health 2018;21:12830.

6. Yung AR, Wood SJ, Malla A et al. The reality of at risk mental state services: a response to recent criticisms. Psychol Med (in press).

7. McHugh MJ, McGorry P, Yuen $\mathrm{H}$ et al. The ultrahigh-risk for psychosis groups: evidence to maintain the status quo. Schizophr Res 2018;195:543-8.

8. Schultze-Lutter F, Klosterkötter J, Gaebel W et al. Psychosis-risk criteria in the general population: frequent misinterpretations and current evidence. World Psychiatry 2018;17:107-8.

9. Perez J, Jones PB. Breaking the web: life beyond the at-risk mental state for psychosis. Psychol Med (in press).

10. Raballo A, Poletti M, Carpenter WT. Rethinking the psychosis threshold in clinical high risk. Schizophr Bull 2019;45:1-2.

11. Moritz S, Gawęda $€$, Heinz A et al. Four reasons why early detection centers for psychosis should be renamed and their treatment targets reconsidered: we should not catastrophize a future we can neither reliably predict nor change. Psychol Med 2019;49:2134-40.

12. Ajnakina O, David AS, Murray RM. 'At risk mental state' clinics for psychosis - an idea whose time has come - and gone! Psychol Med 2019;49:529-34.

13. Nelson B, Amminger GP, McGorry PD. Recent meta-analyses in the clinical high risk for psychosis population: clinical interpretation of findings and suggestions for future research. Front Psychiatry 2018;9:502.
14. McGorry PD, Mei C. Ultra-high-risk paradigm: lessons learnt and new directions. Evid Based Ment Health 2018;21:131-3.

15. Fusar-Poli P. The hype cycle of the clinical high risk state for psychosis: the need of a refined approach. Schizophr Bull 2018;44:250-3.

16. McGorry PD, Hartmann JA, Spooner R et al. Beyond the "at risk mental state" concept: transitioning to transdiagnostic psychiatry. World Psychiatry 2018;17:133-42.

17. Linscott R, Van Os J. An updated and conservative systematic review and meta-analysis of epidemiological evidence on psychotic experiences in children and adults: on the pathway from proneness to persistence to dimensional expression across mental disorders. Psychol Med 2013;43:1133-49.

18. Hanssen M, Bak M, Bijl R et al. The incidence and outcome of subclinical psychotic experiences in the general population. Br J Clin Psychol 2005;44:18191.

19. Bromet EJ, Nock MK, Saha S et al. Association between psychotic experiences and subsequent suicidal thoughts and behaviors: a cross-national analysis from the World Health Organization World Mental Health Surveys. JAMA Psychiatry 2017;74:1136-44

20. Yates K, Lång U, Cederlöf $M$ et al. Association of psychotic experiences with subsequent risk of suicidal ideation, suicide attempts, and suicide deaths: a systematic review and meta-analysis of longitudinal population studies. JAMA Psychiatry 2019;76:180-9.

21. Oh H, Koyanagi A, Kelleher I et al. Psychotic experiences and disability: findings from the Collaborative Psychiatric Epidemiology Surveys. Schizophr Res 2018;193:343-7.

22. Rössler W, Riecher-Rössler A, Angst J et al. Psychotic experiences in the general population: a twenty-year prospective community study. Schizophr Res 2007;92:1-14.

23. Fonville L, Cohen Kadosh K, Drakesmith $\mathrm{M}$ et al. Psychotic experiences, working memory, and the developing brain: a multimodal neuroimaging study. Cereb Cortex 2015;25:4828-38.

24. McGrath JJ, Saha S, Al-Hamzawi A et al. The bidirectional associations between psychotic experiences and DSM-IV mental disorders. Am J Psychiatry 2016;173:997-1006.

25. Kirli U, Binbay T, Drukker M et al. DSM outcomes of psychotic experiences and associated risk factors: 6-year follow-up study in a 
community-based sample. Psychol Med

2019;49:1346-56.

26. Kelleher I, Keeley H, Corcoran P et al. Clinicopathological significance of psychotic experiences in non-psychotic young people: evidence from four population-based studies. $\mathrm{Br} \mathrm{J}$ Psychiatry 2012;201:26-32.

27. Schultze-Lutter F, Michel C, Ruhrmann S et al. Prevalence and clinical relevance of interviewassessed psychosis-risk symptoms in the young adult community. Psychol Med 2018;48:1167-78.

28. de Graaf R, ten Have M, van Dorsselaer S. The Netherlands Mental Health Survey and Incidence Study-2 (NEMESIS-2): design and methods. Int J Methods Psychiatr Res 2010;19:125-41.

29. de Graaf R, ten Have M, van Gool C et al. Prevalence of mental disorders and trends from 1996 to 2009. Results from the Netherlands Mental Health Survey and Incidence Study-2. Soc Psychiatry Psychiatr Epidemiol 2012;47:203-13.

30. de Graaf R, Van Dorsselaer S, Tuithof M et al. Sociodemographic and psychiatric predictors of attrition in the third wave of the Netherlands Mental Health Survey and Incidence Study-2 (NEMESIS-2). Compr Psychiatry 2013;54:1131-9.

31. de Graaf R, Ormel J, Ten Have M et al. Mental disorders and service use in The Netherlands. Results from the European Study of the Epidemiology of Mental Disorders (ESEMeD). New York: Cambridge University Press, 2008.

32. Alonso J, Angermeyer MC, Bernert $\mathrm{S}$ et al Sampling and methods of the European Study of the Epidemiology of Mental Disorders (ESEMeD) project. Acta Psychiatr Scand 2004;109:8-20.

33. Nuyen J, Tuithof M, de Graaf R et al. The bidirectional relationship between loneliness and common mental disorders in adults: findings from a longitudinal population-based cohort study. Soc Psychiatry Psychiatr Epidemiol (in press).

34. Radhakrishnan R, Guloksuz S, Ten Have M et al. Interaction between environmental and familial affective risk impacts psychosis admixture in states of affective dysregulation. Psychol Med 2019;49:1879-89.

35. Reininghaus $\mathrm{U}$, Rauschenberg C, ten Have $\mathrm{M}$ et al Reasoning bias, working memory performance and a transdiagnostic phenotype of affective disturbances and psychotic experiences in the general population. Psychol Med 2019;49:1799809.

36. van Nierop M, Viechtbauer W, Gunther $\mathrm{N}$ et al. Childhood trauma is associated with a specific admixture of affective, anxiety, and psychosis symptoms cutting across traditional diagnostic boundaries. Psychol Med 2015;45:1277-88.

37. Pries L-K, Guloksuz S, ten Have M et al. Evidence that environmental and familial risks for psychosis additively impact a multidimensional subthreshold psychosis syndrome. Schizophr Bull 2018;44:7109.

38. Bak M, Myin-Germeys I, Hanssen M et al. When does experience of psychosis result in a need for care? A prospective general population study. Schizoph Bull 2003;29:349-58.

39. Bijl RV, Ravelli A. Psychiatric morbidity, service use, and need for care in the general population: results of The Netherlands Mental Health Survey and Incidence Study. Am J Public Health 2000;90:602.

40. Pries LK, Lage-Castellanos A, Delespaul P et al. Estimating exposome score for schizophrenia using predictive modeling approach in two independent samples: the results from the EUGEI study. Schizophr Bull 2019;45:960-5.

41. Guloksuz S, Pries LK, Delespaul P et al. Examining the independent and joint effects of molecular genetic liability and environmental exposures in schizophrenia: results from the EUGEI study. World Psychiatry 2019;18:173-82.

42. Efron B. The efficiency of Cox's likelihood function for censored data. J Am Stat Assoc 1977;72:557-65.

43. Lin DY, Wei L-J. The robust inference for the Cox proportional hazards model. J Am Stat Assoc 1989;84:1074-8.

44. Schoenfeld D. Partial residuals for the proportional hazards regression model. Biometrika 1982;69:23941.

45. VanderWeele TJ, Ding P. Sensitivity analysis in observational research: introducing the E-value. Ann Intern Med 2017;167:268-74.

46. Newson RB. Attributable and unattributable risks and fractions and other scenario comparisons. Stata J 2013;13:672-98.

47. Rockhill B, Newman B, Weinberg C. Use and misuse of population attributable fractions. Am J Public Health 1998;88:15-9.

48. Albert U, Tomassi S, Maina G et al. Prevalence of non-psychotic disorders in ultra-high risk individuals and transition to psychosis: a systematic review. Psychiatry Res 2018;270:1-12.

49. Fusar-Poli P, Nelson B, Valmaggia L et al. Comorbid depressive and anxiety disorders in 509 individuals with an at-risk mental state: impact on psychopathology and transition to psychosis. Schizophr Bull 2012;40:120-31. 
50. Addington J, Piskulic D, Liu L et al. Comorbid diagnoses for youth at clinical high risk of psychosis. Schizophr Res 2017;190:90-5.

51. Tanuseputro P, Perez R, Rosella L et al. Improving the estimation of the burden of risk factors: an illustrative comparison of methods to measure smoking-attributable mortality. Popul Health Metr 2015;13:5
52. Jongsma HE, Turner C, Kirkbride JB et al. International incidence of psychotic disorders, 2002-17: a systematic review and meta-analysis. Lancet Public Health 2019;4:e229-44.

53. van Os J, Guloksuz S, Vijn TW et al. The evidencebased group-level symptom-reduction model as the organizing principle for mental health care: time for change? World Psychiatry 2019;18:88-96. 




\section{Abstract:}

Background: The observed link between positive psychotic experiences (PE) and psychosis spectrum disorder (PSD) may be stronger depending on concomitant presence of PE with other dimensions of psychopathology. We examined whether the effect of common risk factors for PSD on PE is additive and whether the impact of risk factors on the occurrence of PE depends on the co-occurrence of other symptom dimensions (affective dysregulation, negative symptoms, and cognitive alteration).

Method: Data from the Netherlands Mental Health Survey and Incidence Study-2 were used. Risk factors included childhood adversity, cannabis use, urbanicity, foreign born, hearing impairment, and family history of affective disorders. Logistic regression models were applied to test (1) the additive effect of risk factors (4 levels) on PE and (2) the moderating effects of symptom dimensions on the association between risk factors (present/absent) and $\mathrm{PE}$, using additive interaction, expressed as the interaction contrast ratio.

Results: Risk factors were additive: the greater the number of risk factors, the greater the odds of PE. Furthermore, concomitant presence of the other symptom dimensions all increased the impact of risk factors on PE. After controlling for age, sex and education, only affective dysregulation and negative symptoms remained significant moderators; only affective dysregulation remained a significant moderator if all dimensions were adjusted for each other.

Conclusions: Risk factors may not be directly associated with PE but additively give rise to a multidimensional subthreshold state anticipating the multidimensional clinical syndrome. Early motivational and cognitive impairments in the context of PE may be reducible to affective dysregulation.

Key words: risk factors, symptom dimensions, psychotic experiences 


\section{Introduction}

The attenuated form of positive psychosis expression, commonly referred to as psychotic experiences $(\mathrm{PE})$, is prevalent in the general population, with rates between $5 \%$ and $8 \%{ }^{1,2}$. It frequently co-occurs with affective dysregulation ${ }^{3,4}$, and predicts both $\mathrm{PSD}^{5-7}$ and mental disorders at large, including mood and anxiety disorders ${ }^{4,8}$. The WHO World Mental Health Surveys demonstrated a bidirectional temporal relation between PE and most mental disorders - the preceding condition increasing the risk of the other over time 9 . Similarly, increased levels of psychosis admixture in nonpsychotic disorders have been observed to impact illness severity ${ }^{10}$, comorbidity ${ }^{3}$, poor outcome ${ }^{11}$, functional impairment ${ }^{12,13}$, and suicidality ${ }^{14,15}$. Furthermore, in a recent study on the distribution of ultra-high risk criteria in the general population, however without applying the help-seeking criterion, the presence of nonpsychotic mental disorders and functional deficits was more likely when attenuated psychotic symptoms co-occurred with cognitive deficits (i.e. cognitive-perceptive basic symptoms and cognitive disturbances $)^{16}$.

The initial manifestation of a mental condition typically represents a mixture of signs and symptoms accompanied by impairment in various emotional and neurocognitive processes that may include aberrant salience, motivational alterations, affective dysregulation and anxiety states ${ }^{17}$. The degree of impairment in these dynamically interacting processes, mediated by underlying biological vulnerabilities, predict the degree of progression from a subtle mental state (subthreshold PE) toward clinical disorder (schizophrenia) ${ }^{17}$. Taken together, these findings suggest that various symptom dimensions and underlying neurocognitive processes co-occur in both clinical and general populations, and interact with each other between traditional diagnostic categories (e.g., depressed mood interact with delusions of reference) $)^{11,18}$ and within traditional diagnostic categories (e.g., auditory hallucinations interact with paranoid ideation) ${ }^{19,20}$.

There is evidence that affective dysregulation and psychosis expression co-occur and share a considerable amount of pathoetiological background ${ }^{21}$. The Bipolar-Schizophrenia Network on Intermediate Phenotypes study yielded neurobiological commonalities cutting across the classical Kraepelinian dichotomy ${ }^{22,23}$, while genome-wide association studies consistently showed significant overlap between affective disorders and $\mathrm{PSD}^{24}$. In agreement with molecular genetic data, we demonstrated that polygenic risk score (PRS) for schizophrenia in healthy participants and nonill relatives of patients with PSD was expressed not only as positive schizotypy but also in the domains of affective regulation, neurocognition, and attribution of salience ${ }^{25}$. Furthermore, PRS for schizophrenia was associated with lifetime mood episodes (both depressive and manic) in relatives and healthy controls ${ }^{25}$. Confirming 
the shared vulnerability theory, environmental exposure (like cannabis use, childhood adversity, urbanicity, and hearing impairment) likewise is associated with psychosis and with affective and stress-related phenotypes ${ }^{26-29}$.

It is therefore reasonable to hypothesize that environmental exposure, along with genetic vulnerability, may synergistically alter the degree of blending of affective dysregulation and psychosis expression, thus triggering progression toward a more serious clinical condition. Converging evidence indicates that the degree of psychotic admixture in affective disorders is contingent on the level of environmental exposure that is linked to PSD, such as cannabis use $^{30}$ and childhood trauma ${ }^{31,32}$. Previously, our group demonstrated that both childhood adversity and cannabis exposure additively increased the likelihood of admixture of psychosis expression in affective disorders in a dose-response fashion ${ }^{33}$, and that the level of connectivity between different psychopathological dimensions increased as a function of the environmental risk load ${ }^{34}$.

In line with our multidimensional approach to explore the impact of various risk factors on psychopathology in the general population, this study aimed to investigate to what degree the association between common risk factors for PSD (childhood adversity, cannabis use, urbanicity, foreign born, hearing impairment and family history of affective disorders) and $\mathrm{PE}$ is contingent on other components of the multidimensional psychosis spectrum (affective dysregulation, negative symptoms, and cognitive alteration) in the general population. The study also aimed to clarify to what degree risk factors are additive (linearly increasing) or redundant (not adding to each other).

\section{Method}

\section{Study population}

Data were obtained from the Netherlands Mental Health Survey and Incidence Study-2 (NEMESIS-2) designed to investigate the prevalence, incidence, course, and consequences of mental disorders in the Dutch general population. The baseline data of NEMESIS-2 were collected from 2007 to 2009. The study was approved by the Medical Ethics Review Committee for Institutions on Mental Health Care and written informed consent was collected from participants. To ensure representativeness of the sample in terms of age (between the ages of 18-65 at baseline), region, and population density, a multistage random sampling procedure was applied ${ }^{35}$. Dutch literacy was an inclusion criterion. Nonclinician, trained interviewers applied the Composite International Diagnostic Interview (CIDI) version 3.0 and additional questionnaires during home visits. Details of NEMESIS-2 were provided elsewhere ${ }^{35,36}$. The first wave (T0) enrolled 6,646 participants (response rate $65.1 \%$; 
average duration: 95 minutes), who were followed up in 2 visits within 6 years: successive response rates at year 3 (T1) and at year 6 (T2) were $80.4 \%(n=5,303$; excluding those who deceased; duration: 84 minutes) and $87.8 \%(\mathrm{n}=4,618$; duration: 83 minutes), respectively. Data from all waves were utilized. Rates at baseline reflect lifetime occurrence; rates at $\mathrm{T} 1$ and T2 reflect interval (baseline-T1 and T1-T2) occurrence. Attrition between T0 and T1 ${ }^{37}$ and between $\mathrm{T} 1$ and $\mathrm{T} 2^{38}$ was not significantly associated with any of the 12-month mental disorders at T0 controlled for sociodemographics, except for alcohol and drug dependence at $\mathrm{T} 1$ which were significantly related with attrition at $\mathrm{T} 2^{38}$.

\section{Dimensions of psychopathology}

\section{Psychotic experiences}

To assess PE, a questionnaire constructed based on CIDI 1.1 was used. Participants were asked at baseline (T0; life time symptoms) and at follow-ups (T1 and T2; 3-year symptoms) whether they had experienced any of a list of 20 positive psychotic symptoms (with a binary response: $0=$ "no" and 1="yes"; items are listed in supplementary table $S 1)^{32,39}$. For the purpose of this analysis, a dichotomous $\mathrm{PE}$ variable was defined as positive if any of the positive symptom items were rated positively.

\section{Affective dysregulation}

The CIDI 3.0 was used to assess depressive, manic, and anxiety symptoms. Affective dysregulation was considered present when participants endorsed at least one of the CIDI 3.0 core symptoms of Depressive Episode, Panic Disorder, Social Phobia, Generalized Anxiety Disorder, and Manic Episode. Affective dysregulation was assessed at each timepoint (T0, T1 and T2).

\section{Negative symptoms}

The negative symptom dimension was constructed using 4 items based on interviewer observation: poor personal hygiene and inadequate independent living skills (i.e., neatness and cleanness of the participant's residence; were assessed at T1 and T2); lack of emotional expression and poverty of speech (were assessed at T2). Presence of negative symptoms was defined as a rating of "present" on any of these items, which were dichotomized as present = " 0 " and absent $=$ " 1 ". Presence of negative symptoms at T1 and/or T2 was used as a personlevel indicator of "trait" negative symptom at T0.

\section{Cognitive alteration}

The forward and backward digit span tasks from the Wechsler Adult Intelligence Scale$\mathrm{III}^{40}$ was used to assess short-term attention and working memory performance, respectively, at T1. A binary cognitive alteration variable was constructed using a cut-off point to define the group of participants with the lowest $20 \%$ raw scores of combined forward and backward 
tasks of digit span. Presence of cognitive alteration at T1 was used as a person-level trait indicator of cognitive alteration at T0 and T2.

\section{Risk factors}

\section{Family history of affective disorders}

Family history includes depressive, manic and anxiety items (panic disorder, specific phobia, social phobia, agoraphobia, generalized anxiety disorder) as well as items on drugs and alcohol use. Assessment of family history was limited to the participants who screened positive for affective disorders: depression, mania, and anxiety disorders (panic disorder, specific phobia, and generalized anxiety disorder).

\section{Childhood adversity}

Childhood adversity (CA) was assessed at baseline (T0) across 5 domains (emotional neglect, peer victimization, physical abuse, psychological abuse, and sexual abuse; before age 16) using a questionnaire based on the NEMESIS-1 trauma questionnaire ${ }^{35}$. Subjects were asked whether they experienced emotional neglect, psychological abuse, peer victimization or physical abuse on $\geq 2$ occasions, or sexual abuse on $\geq 1$ occasion. A person-level continuous CA variable was constructed using the sum score of the 5 domains. In accordance with previous research ${ }^{41}$, CA was dichotomized at the 80 th percentile.

\section{Cannabis exposure}

Cannabis use was assessed with the section Illegal Substance Use of the CIDI 3.0 at baseline (T0; lifetime) and at follow-ups (T1 and T2; 3-year). If subjects reported cannabis use, they were rated on frequency of use in the period of most frequent use on a scale of 1 (never) to 7 (every day). Consistent with previous work ${ }^{42}$, a binary variable was constructed by using the cut-off value of once per week or more in the period most frequent use.

\section{Urban environment}

Exposure to an urban environment until age 16 years was assessed at baseline (T0) and analyzed as a person-level variable across the 3 waves at 5 levels: 1$)$ countryside (distances to amenities is bigger), 2) village ( $<25,000$ inhabitants), 3) small city $(25,000-50,000$ inhabitants), 4) medium city (50,000-100,000 inhabitants), 5) large city ( $>100,000$ inhabitants). Conforming to previous work using the NEMESIS-I dataset ${ }^{34}$, the cut-off of more than 50.000 inhabitants was used to define the binary variable of urban area.

\section{Foreign born}

Country of birth was assessed at baseline (T0) and analyzed as a person-level variable across the 3 waves. It was used as a proxy for minority status (born in the Netherlands = " 0 " and born in other countries $=$ " 1 ”). 


\section{Hearing impairment}

Hearing impairment was assessed at each time-point (T0, T1 and T3), based on self-reported hearing impairment in the last 12 months (absent = "0" and present = " 1 ").

\section{Statistical Analysis}

Analyses were performed using Stata $14.2^{43}$. Consistent with previous work ${ }^{33}$, data from all waves were analyzed cross-sectionally in the "long format" (each participant contributing 3 observations: T0, T1, and T2). This analytical strategy serves the purposes of increasing overall reliability and achieving consistency across a limited number of variables of interest that in some cases were assessed differently at different time points. Using the CLUSTER option, all analyses were corrected for clustering of multiple observations within subjects, and cluster-robust standard errors were computed. To evaluate the effect of the risk-loading on $\mathrm{PE}$, logistic regression analysis, using the LOGISTIC command, was modelled with PE as dependent variable and the 4-level risk score $($ no $=0$, low $=1$, medium $=2$, and high $>2$ risk factors) as independent variable. The model was adjusted for sex, age, and education ( $1=$ primary school, 2 = lower secondary education, 3 = higher secondary education, 4 = higher professional education). The LINCOM command was applied to test OR differences between groups with low and medium, medium and high, and low and high risk.

Logistic regression models were used to analyze whether the association between the presence of any risk factors (absent $=0$; presence of one or more risk factors $=1$ ) and PE was dependent on symptom dimensions (affective dysregulation, negative symptoms, and cognitive alteration). The interaction contrast ratio (ICR) method was applied to explore the interaction between risk factors and symptom dimensions in the model of PE. To test additive interaction, 4 exposure states were produced by the combination of each dimension and risk. In logistic models, the combinations served as the independent variables (3 dummy variables with nonexposed state as the reference category) and PE served as dependent variable. Using the ORs from these models, ICRs for each model were calculated using the NLCOM command in Stata: e.g., ICR = OR (risk) + OR (affective dysregulation) - OR (risk) - OR (affective dysregulation) +1 . These models were further adjusted for age (continuous), sex, and education level (4 level). In the final adjusted models, psychopathology dimensions were additionally controlled for each other.

\section{Results}

The total sample for the analyses included 16,567 observations from subjects who participated at the 3 time-points ( $\mathrm{T} 0, \mathrm{n}=6,646 ; \mathrm{T} 1, \mathrm{n}=5,303 ; \mathrm{T} 2, \mathrm{n}=4,618)$. The 
baseline demographics of the NEMESIS-2 sample have been described previously ${ }^{32}$ and an overview of the 3 time-points is presented in Table 1 . Supplementary table S1 reports the frequencies of individual $\mathrm{PE}$ items at each time-point.

Table 1. Summary of descriptive data

\begin{tabular}{lccc}
\hline & Baseline & 3-year follow-up & 6-year follow-up \\
& $\mathrm{n}=6,646$ & $\mathrm{n}=5,303$ & $\mathrm{n}=4,618$ \\
\hline Sex, female & $3,672(55.3)$ & $2,922(55.1)$ & $2,558(55.4)$ \\
Mean age, years (SD) & $44.3(12.5)$ & $47.6(12.4)$ & $50.9(12.3)$ \\
Education & & & $186(4)$ \\
$\quad$ Primary education & $332(5)$ & $226(4.3)$ & $1,193(25.8)$ \\
$\quad$ Lower secondary education & $1,826(27.5)$ & $1,388(26.2)$ & $1,479(32)$ \\
$\quad$ Higher secondary education & $2,145(32.3)$ & $1,728(32.6)$ & $1,760(38.1)$ \\
$\quad$ Higher professional education, university degree & $2,343(35.3)$ & $1,961(37)$ & $529(11.5)$ \\
$\quad$ Foreign born & $920(13.8)$ & $650(12.3)$ & 5 \\
\hline Data are given as number (percentage) unless otherwise indicated. & &
\end{tabular}

\section{Dose-response relationship between the risk-loading and PE}

Analyzing environmental and familial risk load at 4 levels (no $=0$, low $=1$, medium $=2$, and high $>2$ risk factors), irrespective of PSD dimensions, revealed a dose-response relationship between risk and PE: With "no exposure" as the reference group, risk categories displayed progressively higher odds ratios: $\mathrm{OR}=1.84,95 \% \mathrm{CI}=1.58-2.13, P<0.001$ for the low risk group; $\mathrm{OR}=3.11,95 \% \mathrm{CI}=2.64-3.66, \mathrm{p}<0.001$ for the medium risk group; $\mathrm{OR}=5.78$, $95 \% \mathrm{CI}=4.77-7, \mathrm{p}<0.001$ for the high risk group. Furthermore, comparison with the LINCOM command indicated significant differences between the groups with low and medium risk $(\mathrm{OR}=1.7,95 \% \mathrm{CI}=1.47-1.94, P<0.001)$, medium and high risk $(\mathrm{OR}=$ $1.86,95 \% \mathrm{CI}=1.56-2.22, P<0.001)$, and low and high risk $(\mathrm{OR}=3.1595 \% \mathrm{CI}=2.64$ $3.74, P<0.001)$. Figure 1 shows the frequencies of individual risk factors within risk strata (no $=0$, low $=1$, medium $=2$, and high $>2$ risk factors) at baseline level. Figure 2 shows the prevalence of $\mathrm{PE}$ across the risk strata, including data from participants with information from all time-points.

\section{Testing the moderating effects of affective dysregulation}

The association between risk and PE was greater if there was also evidence for affective dysregulation (Table 2). Figure 3a shows that the adjusted OR for those with affective dysregulation and risk factors was 6.29, in comparison with ORs of 1.27 for those with risk factors only, and 3.04 for those with affective dysregulation only, yielding an ICR of 2.98 ( $P$ $<0.001)$. The additive effect of affective dysregulation remained significant after the other dimensions of psychopathology (negative symptoms, cognitive alteration) were controlled for, with an ICR of $2.74(95 \% \mathrm{CI}=1.92-3.55, P<0.001)$. 
Figure 1. The frequencies of risk factors within risk factor strata at baseline assessment

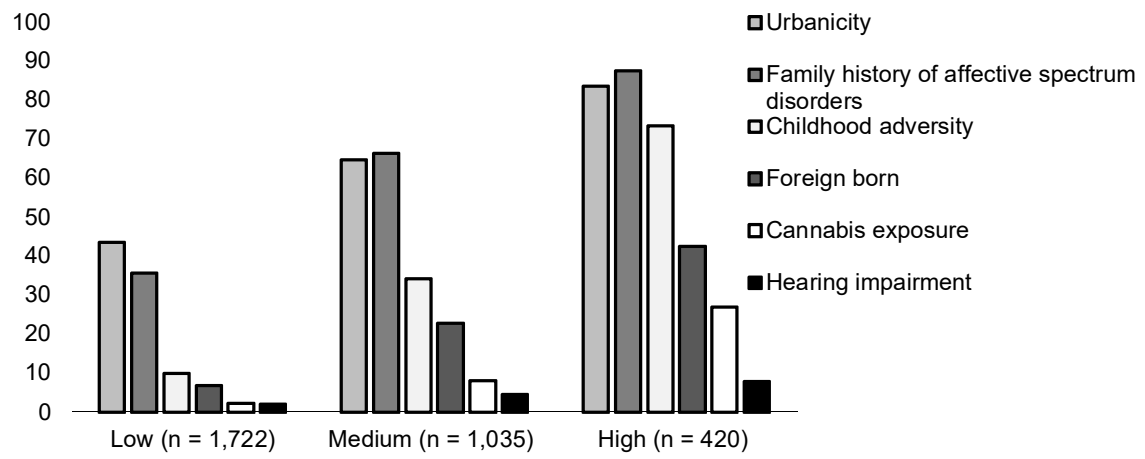

Figure 1 shows the frequencies of individual risk factors in each risk stratum at baseline: No risk factor $=0$, low risk $=1$ risk factor, medium risk $=2$ risk factors, and high risk $>2$ risk factors. Data were given in percentages based on the individual sample sizes of the low, medium, and high risk groups, respectively

\section{Testing the moderating effects of negative symptoms}

The association between risk and PE was greater if there was also evidence for negative symptoms (Table 2 ). Figure $3 \mathrm{~b}$ shows that the adjusted OR for those with negative symptoms and risk factors was 5.35, in comparison with ORs of 2.45 for those with risk factors only, and 1.69 for those with negative symptoms only, yielding an ICR of $2.20(P=0.004)$. After controlling for the other dimensions of psychopathology (affective dysregulation, cognitive alteration), there was no significant additive effect $(\mathrm{ICR}=-0.35,95 \% \mathrm{CI}=-1.09-0.39, P=$ $0.36)$.

Table 2. Additive effect of dimensional components on the association between risk factors and psychosis expression

\begin{tabular}{|c|c|c|c|c|c|c|c|c|c|}
\hline & \multicolumn{3}{|c|}{ Unadjusted model } & \multicolumn{3}{|c|}{ Adjusted model ${ }^{\mathrm{a}}$} & \multicolumn{3}{|c|}{$\begin{array}{c}\text { Adjusted model }{ }^{\mathrm{a}} \\
\text { (corrected for the other dimensions) }\end{array}$} \\
\hline & ICR & $95 \% \mathrm{CI}$ & $\mathrm{p}$ & ICR & $95 \% \mathrm{CI}$ & $\mathrm{p}$ & ICR & $95 \% \mathrm{CI}$ & $P$ \\
\hline $\begin{array}{l}\text { Affective } \\
\text { dysregulation }\end{array}$ & 3.18 & $2.37 ; 3.98$ & $<0.001$ & 2.98 & $2.21 ; 3.75$ & $<0.001$ & 2.74 & $1.92 ; 3.55$ & $<0.001$ \\
\hline $\begin{array}{l}\text { Negative } \\
\text { symptoms }\end{array}$ & 2.68 & $1.13 ; 4.23$ & 0.001 & 2.20 & $0.71 ; 3.69$ & 0.004 & -0.35 & $-1.09 ; 0.39$ & 0.355 \\
\hline $\begin{array}{l}\text { Cognitive } \\
\text { alteration }\end{array}$ & 0.74 & $0.03 ; 1.45$ & 0.042 & 0.55 & $-0.16 ; 1.26$ & 0.128 & -0.28 & $-0.71 ; 0.15$ & 0.199 \\
\hline
\end{tabular}

${ }^{a}$ Adjusted for age, sex, and education; controlled ICR, Interaction contrast ratio; CI, confidence interval. 


\section{Testing the moderating effects of cognitive alteration}

The association between risk factors and $\mathrm{PE}$ was greater if there was also evidence for cognitive alteration (Table 2). However, the additive effect was no longer significant in either the adjusted model (Figure 3c) nor in the model controlled for the other symptom dimensions (negative symptoms, affective dysregulation; ICR $=-0.28,95 \%$ $\mathrm{CI}=-0.71-0.15, P=0.20)$.

\section{Discussion}

We explored the possible existence of a risk-loading effect on PE and investigated whether the association between the risk for PSD and PE was contingent on multidimensional psychopathology (affective dysregulation, negative symptoms, and cognitive alteration). The findings were that: (1) risk factors additively increased the likelihood of PE in a dose-response fashion; (2) affective dysregulation, negative symptoms, and cognitive alteration additively increased the association between risk factors and PE; (3) the association of risk factors with affective dysregulation and negative symptoms remained significant in the model adjusted for age, sex, and education; (4) the association of risk factors with affective dysregulation remained significant in the final model, when all PSD dimensions were controlled for each other.

\section{Multidimensional psychopathology}

There is growing evidence that psychosis expression represents a severity indicator for multidimensional psychopathology cutting across traditional diagnostic boundaries ${ }^{8,14,44}$, and that nonpsychotic symptoms, such as affective dysregulation ${ }^{12,45}$, negative symptoms ${ }^{13,46}$, and neurocognitive alteration ${ }^{13,46,47}$ precede the early stages of PSD and predict progression to more severe states. The current population-based study provides additional support for this concept by showing that epidemiological risk factors for PSD are not exclusively associated with PE, but rather with the degree of amalgamated multidimensional psychopathology ${ }^{48-50}$. 
Our findings are in agreement with the literature showing symptom dimensions interrelate within ${ }^{19,20}$ and between ${ }^{11,18}$ different diagnoses, and that the severity of clinical outcomes of diverse clinical representations may depend on the degree of interconnection between those dimensions ${ }^{7,13,20,51}$. For instance, the presence of PE in anxiety or depression disorders was shown to predict severity of clinical outcomes and treatment response $e^{7,52,53}$; and by investigating fluctuations of momentary mental states (e.g., paranoia, positive, and negative affect) in daily life, studies using the experience sampling methodology found that an increased connectivity between momentary states was associated with symptom severity and need for care ${ }^{54-56}$.

Consistent with previous research, current findings suggest that risk factors operate by intensifying the multidimensional blending of affective and cognitive processes ${ }^{51,57-60}$, as well as the negative symptom domain ${ }^{13,61}$ in the development of PSD. This finding fits well with our previous findings showing that the risk-loading (childhood trauma, urbanicity, cannabis use, and discrimination) amplifies connectivity between different symptom dimensions ${ }^{34}$. Similarly, exposure to cannabis use and childhood trauma was found to increase associations between hallucination and delusion in healthy and in genetically at risk populations ${ }^{18,19}$; while another study reported that exposure to childhood trauma had a stronger correlation with a combined symptom network rather than the individual symptoms ${ }^{32}$. Our finding that only the effect of affective dysregulation remained significant after the adjustment for the other dimensions is furthermore compatible with the theory of an "affective path to psychosis" ${ }^{\prime 7,62}$.

\section{The risk-loading in the context of liability threshold model}

The results echo findings from previous research showing a dose-response relationship between risk factors and $\mathrm{PE}^{33,63}$, emphasizing the effects of risk-loading for PSD. Recently, researchers attempted to construct a "polyenviromic risk score" (PERS) for psychosis (the sum of weighted scores of known environmental risk factors based on their association with psychosis reported in meta-analyses). Despite several methodological issues, this proof-ofconcept study showed that higher PERS predicted greater risk of developing psychosis in genetically at-risk individuals ${ }^{64}$. Additionally, recent studies of individualized risk calculators for psychosis focusing on demographic, clinical, and some environmental predictors may provide insight into estimating psychosis risk in clinical settings ${ }^{65,66}$. 


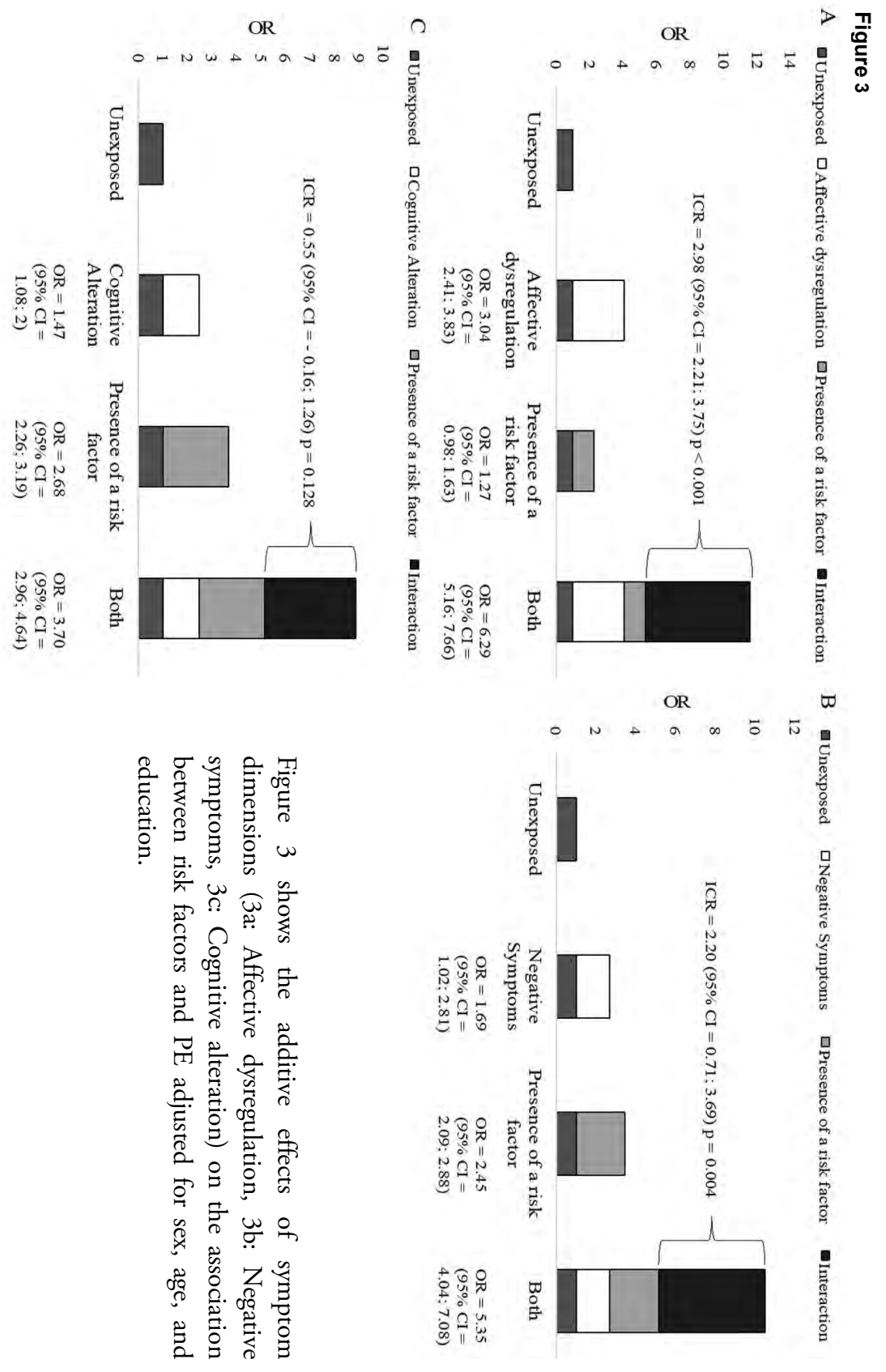


It is plausible to argue that the mechanisms underlying the development of PSD may be best understood in the context of the liability-threshold model ${ }^{67}$ that posits the combination of various genetic and environmental factors — with each factor adding to the risk load — adding to the manifestation of a phenotypic outcome. The distribution of liability may not be continuous, as the apparent phenomenological and temporal continuity of psychotic experiences with PSD may in fact reflect an underlying discontinuous population distribution consisting of vulnerable and nonvulnerable individuals ${ }^{68,69}$.

To further investigate environmental and genetic risk loading, future studies should focus on constructing reproducible total scores of environmental exposures, along with a single metric of aggregated molecular genetic variation (PRS), to disentangle the additive effects of geneenvironment interplay on the development and course of PSD.

Given the complexity of multi-dimensional psychopathology, the network approach to symptoms ("symptomics"), a rapidly evolving analytical strategy, may also provide an alternative platform to gain insight into the role of gene-environment interplay in the development and progression of PSD, with initial findings showing (at least) some promise but requiring replication ${ }^{70,71}$.

\section{Limitations}

The primary strength of this study was the multi-domain clinical phenotyping and the use of a large and representative population cohort collected at 3 time-points over 6 years. However, various methodological limitations should be considered when interpreting the findings.

First, the cross-sectional analysis of the dataset merging data from each time-point in the "long-format," while decreasing threats to external validity, cannot be taken as absolutely confirmatory of causality ${ }^{72}$. Ideally, a time series analysis of a birth cohort—followed up with regular in-depth assessments at short-enough intervals to capture emerging psychopathology stretching over the period at risk for emerging mental disorders — is required to yield essential information to understand the impact of psychosis liability on the emotional, behavioral, and cognitive components of PSD. With no such data available now or in the near future, our practical strategy, despite its shortcomings, expands our knowledge-base, implying that the association between psychosis risk and psychosis expression is moderated by existing multidimensional psychopathology. 
Second, although the dataset includes a fine-grained assessment of positive PE and affective symptomatology, there were only 4 proxy items appraising the negative symptom dimension. A more thorough assessment, using a validated rating scale with focus on measuring the negative symptom dimension as well as PE in the general population (such as the Structured Interview for Schizotypy-Revised (SIS-R) and the Community Assessment of Psychic Experiences (CAPE)) could have been beneficial in capturing the negative symptom dimension across the full range.

Third, our approach to risk stratification through aggregating vulnerability factors assumes a linear increase in the risk for psychosis as a function of the number of risk factors and weighs each risk factors equally by overlooking any specific feature pertaining to individual risk factors and their synergistic effects.

Although current findings are in line with those of previous studies in different populationbased datasets, replication is necessary. We aim for a reproducing the findings in the European network of national schizophrenia networks studying gene-environment interaction dataset, which includes heterogeneous, international, multi-ethnic samples of patients, relatives and healthy controls ${ }^{73,74}$.

\section{Conclusion}

Consistent with previous findings, this study demonstrates that the association between psychosis expression and risk-loading (environmental and familial) is contingent on the dimensions of PSD, lending further support to the framework of an affective path to psychosis. Also, as predicted by the liability-threshold model, in which vulnerability for a phenotypic outcome can be modeled as a continuous metric of quantifiable risk, the aggregated risk-loading increased the odds of psychosis expression in a dose-response fashion.

Overall, our recent findings, combined with strong evidence from unbiased population-based cohorts, demonstrate the need for reconstructing the framework of psychosis by integrating multidimensional measurement of psychopathology to advance our understanding of the complex network of biopsychosocial mechanisms underlying the early progression of psychopathology and to dissect diverse developmental paths to psychosis ${ }^{75}$. 


\section{Funding}

Financial support has been received from the Ministry of Health, Welfare and Sport, with supplementary support from the Netherlands Organization for Health Research and Development (ZonMw) and the Genetic Risk and Outcome of Psychosis (GROUP) investigators. These funding sources had no further role in study design; in the collection, analysis and interpretation of data; in the writing of the report; or in the decision to submit the paper for publication. Grants were received to write this paper on data from NEMESIS: Supported by the European Community's Seventh Framework Program under Grant agreement No. HEALTH-F2-2009-241909 (Project EU-GEI).

\section{Acknowledgment}

NEMESIS-2 is conducted by the Netherlands Institute of Mental Health and Addiction (Trimbos Institute) in Utrecht. 


\section{References}

1. van Os J, Linscott RJ, Myin-Germeys I, Delespaul P, Krabbendam L. A systematic review and metaanalysis of the psychosis continuum: evidence for a psychosis proneness-persistence-impairment model of psychotic disorder. Psychol Med. 2009;39(2):179-195.

2. Linscott RJ, van Os J. An updated and conservative systematic review and meta-analysis of epidemiological evidence on psychotic experiences in children and adults: on the pathway from proneness to persistence to dimensional expression across mental disorders. Psychol Med. 2013;43(6):1133-1149.

3. DeVylder JE, Burnette D, Yang LH. Co-occurrence of psychotic experiences and common mental health conditions across four racially and ethnically diverse population samples. Psychol Med. 2014;44(16):3503-3513.

4. Kaymaz N, van Os J, de Graaf R, Ten Have M, Nolen W, Krabbendam L. The impact of subclinical psychosis on the transition from subclinicial mania to bipolar disorder. J Affect Disord. 2007;98(1-2):55-64.

5. Dominguez $\mathrm{M}$, Wichers $\mathrm{M}$, Lieb R, Wittchen $\mathrm{H}$ $\mathrm{U}$, van Os J. Evidence that onset of clinical psychosis is an outcome of progressively more persistent subclinical psychotic experiences: an 8year cohort study. Schizophr Bull 2009;37(1):8493.

6. Kaymaz N, Drukker M, Lieb R, et al. Do subthreshold psychotic experiences predict clinical outcomes in unselected non-help-seeking population-based samples? A systematic review and meta-analysis, enriched with new results. Psychol Med. 2012;42(11):2239-2253.

7. Wigman JT, van Nierop M, Vollebergh WA, et al. Evidence that psychotic symptoms are prevalent in disorders of anxiety and depression, impacting on illness onset, risk, and severity--implications for diagnosis and ultra-high risk research. Schizophr Bull. 2012;38(2):247-257.

8. Rossler W, Hengartner MP, Ajdacic-Gross V, Haker H, Gamma A, Angst J. Sub-clinical psychosis symptoms in young adults are risk factors for subsequent common mental disorders. Schizophr Res. 2011;131(1-3):18-23.

9. McGrath JJ, Saha S, Al-Hamzawi A, et al. The Bidirectional Associations Between Psychotic Experiences and DSM-IV Mental Disorders. The American journal of psychiatry. 2016;173(10):9971006.
10. Armando M, Lin A, Girardi P, et al. Prevalence of psychotic-like experiences in young adults with social anxiety disorder and correlation with affective dysregulation. J Nerv Ment Dis. 2013;201(12):1053-1059.

11. Wigman JT, van Os J, Abidi L, et al. Subclinical psychotic experiences and bipolar spectrum features in depression: association with outcome of psychotherapy. Psychol Med. 2014;44(2):325-336.

12. Fusar-Poli P, Nelson B, Valmaggia L, Yung AR, McGuire PK. Comorbid depressive and anxiety disorders in 509 individuals with an at-risk mental state: impact on psychopathology and transition to psychosis. Schizophr Bull. 2014;40(1):120-131.

13. Dominguez MD, Saka MC, Lieb R, Wittchen HU, van Os J. Early expression of negative/disorganized symptoms predicting psychotic experiences and subsequent clinical psychosis: a 10-year study. The American journal of psychiatry. 2010;167(9):10751082.

14. Kelleher I, Devlin N, Wigman JT, et al. Psychotic experiences in a mental health clinic sample: implications for suicidality, multimorbidity and functioning. Psychol Med. 2014;44(8):1615-1624.

15. Honings $S$, Drukker $M$, van Nierop $M$, et al. Psychotic experiences and incident suicidal ideation and behaviour: Disentangling the longitudinal associations from connected psychopathology. Psychiatry Res. 2016;245:267-275.

16. Schultze-Lutter F, Michel C, Ruhrmann S, Schimmelmann BG. Prevalence and clinical relevance of interview-assessed psychosis-risk symptoms in the young adult community. Psychological medicine. 2017:1-15.

17. van Os J. The dynamics of subthreshold psychopathology: implications for diagnosis and treatment. The American journal of psychiatry. 2013;170(7):695-698

18. Smeets F, Lataster T, Dominguez MD, et al. Evidence that onset of psychosis in the population reflects early hallucinatory experiences that through environmental risks and affective dysregulation become complicated by delusions. Schizophr Bull. 2012;38(3):531-542.

19. Smeets F, Lataster T, Viechtbauer W, Delespaul P, GROUP. Evidence that environmental and genetic risks for psychotic disorder may operate by impacting on connections between core symptoms of perceptual alteration and delusional ideation. Schizophr Bull. 2014;41(3):687-697.

20. Smeets F, Lataster T, van Winkel R, de Graaf R, Ten Have M, van Os J. Testing the hypothesis that 
psychotic illness begins when subthreshold hallucinations combine with delusional ideation. Acta Psychiatr Scand. 2013;127(1):34-47.

21. Upthegrove R, Marwaha S, Birchwood M. Depression and Schizophrenia: Cause, Consequence, or Trans-diagnostic Issue? Schizophr Bull. 2017;43(2):240-244.

22. Sheffield JM, Kandala S, Tamminga CA, et al. Transdiagnostic Associations Between Functional Brain Network Integrity and Cognition. JAMA psychiatry. 2017;74(6):605-613.

23. Ivleva EI, Bidesi AS, Keshavan MS, et al. Gray matter volume as an intermediate phenotype for psychosis: Bipolar-Schizophrenia Network on Intermediate Phenotypes (B-SNIP). The American journal of psychiatry. 2013;170(11):1285-1296.

24. Cross-Disorder Group of the Psychiatric Genomics C, Lee SH, Ripke S, et al. Genetic relationship between five psychiatric disorders estimated from genome-wide SNPs. Nat Genet. 2013;45(9):984994.

25. van Os J, van der Steen Y, Islam MA, et al. Evidence that polygenic risk for psychotic disorder is expressed in the domain of neurodevelopment, emotion regulation and attribution of salience. Psychol Med. 2017;47(14):2421-2437.

26. Misiak B, Krefft M, Bielawski T, Moustafa AA, Sasiadek MM, Frydecka D. Toward a unified theory of childhood trauma and psychosis: A comprehensive review of epidemiological, clinical, neuropsychological and biological findings. Neurosci Biobehav Rev. 2017;75:393-406.

27. Radhakrishnan R, Wilkinson ST, D'Souza DC. Gone to Pot - A Review of the Association between Cannabis and Psychosis. Frontiers in psychiatry. 2014;5:54.

28. Heinz A, Deserno L, Reininghaus U. Urbanicity, social adversity and psychosis. World Psychiatry. 2013;12(3):187-197.

29. Gevonden MJ, Myin-Germeys I, van den Brink W, van Os J, Selten JP, Booij J. Psychotic reactions to daily life stress and dopamine function in people with severe hearing impairment. Psychol Med. 2015;45(8):1665-1674.

30. Reeves LE, Anglin DM, Heimberg RG, et al. Anxiety mediates the association between cannabis use and attenuated positive psychotic symptoms. Psychiatry Res. 2014;218(1-2):180-186.

31. Catalan A, Angosto V, Diaz A, et al. Relation between psychotic symptoms, parental care and childhood trauma in severe mental disorders. Psychiatry Res. 2017;251:78-84.

32. van Nierop M, Viechtbauer W, Gunther N, et al. Childhood trauma is associated with a specific admixture of affective, anxiety, and psychosis symptoms cutting across traditional diagnostic boundaries. Psychol Med. 2015;45(6):1277-1288

33. Guloksuz S, van Nierop M, Lieb R, van Winkel R, Wittchen HU, van Os J. Evidence that the presence of psychosis in non-psychotic disorder is environment-dependent and mediated by severity of non-psychotic psychopathology. Psychol Med. 2015;45(11):2389-2401.

34. Guloksuz S, van Nierop M, Bak M, et al. Exposure to environmental factors increases connectivity between symptom domains in the psychopathology network. BMC Psychiatry. 2016;16(1):223.

35. de Graaf R, Ten Have M, van Dorsselaer $S$. The Netherlands Mental Health Survey and Incidence Study-2 (NEMESIS-2): design and methods. Int J Methods Psychiatr Res. 2010;19(3):125-141.

36. de Graaf R, ten Have M, van Gool C, van Dorsselaer S. Prevalence of mental disorders and trends from 1996 to 2009. Results from the Netherlands Mental Health Survey and Incidence Study-2. Soc Psychiatry Psychiatr Epidemiol. 2012;47(2):203-213.

37. de Graaf R, van Dorsselaer S, Tuithof M, ten Have M. Sociodemographic and psychiatric predictors of attrition in a prospective psychiatric epidemiological study among the general population. Result of the Netherlands Mental Health Survey and Incidence Study-2. Compr Psychiatry. 2013;54(8):1131-1139.

38. De Graaf R, Van Dorsselaer S, Tuithof M, ten Have M. Sociodemographic and psychiatric predictors of attrition in the third wave of the Netherlands Mental Health Survey and Incidence Study-2 (NEMESIS-2). Utrecht: Trimbosinstituut. 2015.

39. Andrews G, Peters L. The psychometric properties of the Composite International Diagnostic Interview. Soc Psychiatry Psychiatr Epidemiol. 1998;33(2):80-88.

40. Wechsler D. Wechsler Adult Intelligence ScaleThird Edition. San Antonio, Texas: The Psychological Corporation; 1997.

41. van Dam DS, van Nierop M, Viechtbauer W, et al. Childhood abuse and neglect in relation to the presence and persistence of psychotic and depressive symptomatology. Psychol Med. 2015;45(7):13631377.

42. van Winkel R, van Beveren NJ, Simons C, Genetic $\mathrm{R}$, Outcome of Psychosis I. AKT1 moderation of cannabis-induced cognitive alterations in psychotic disorder. Neuropsychopharmacology. 2011;36(12):2529-2537. 
43. Stata Statistical Software [computer program]. Version 14. College Station, TX: StataCorp LP; 2015.

44. Navarro-Mateu F, Alonso J, Lim C, et al. The association between psychotic experiences and disability: results from the WHO World Mental Health Surveys. Acta Psychiat Scand. 2017;136(1):74-84.

45. Hafner H, Maurer K, Trendler G, an der Heiden W, Schmidt M, Konnecke R. Schizophrenia and depression: challenging the paradigm of two separate diseases--a controlled study of schizophrenia, depression and healthy controls. Schizophr Res. 2005;77(1):11-24.

46. Hafner H, Loffler W, Maurer K, Hambrecht M, an der Heiden W. Depression, negative symptoms, social stagnation and social decline in the early course of schizophrenia. Acta Psychiatr Scand. 1999;100(2):105-118.

47. Reichenberg A, Caspi A, Harrington H, et al. Static and dynamic cognitive deficits in childhood preceding adult schizophrenia: a 30-year study. Am J Psychiat. 2009;167(2):160-169.

48. van Os J, Guloksuz S. A critique of the 'ultra-high risk' and 'transition' paradigm. World Psychiatry. 2017;16(2):200-206.

49. van Os J, Reininghaus U. Psychosis as a transdiagnostic and extended phenotype in the general population. World Psychiatry. 2016;15(2):118-124.

50. Reininghaus U, Bohnke JR, Hosang G, et al. Evaluation of the validity and utility of a transdiagnostic psychosis dimension encompassing schizophrenia and bipolar disorder. Br J Psychiatry. 2016;209(2):107-113.

51. Freeman D, Dunn G, Fowler D, et al. Current paranoid thinking in patients with delusions: the presence of cognitive-affective biases. Schizophr Bull. 2013;39(6):1281-1287.

52. Kelleher I, Keeley H, Corcoran P, et al. Clinicopathological significance of psychotic experiences in non-psychotic young people: evidence from four population-based studies. $\mathrm{Br} J$ Psychiatry. 2012;201(1):26-32.

53. Jeppesen P, Clemmensen L, Munkholm A, et al. Psychotic experiences co-occur with sleep problems, negative affect and mental disorders in preadolescence. J Child Psychol Psychiatry. 2015;56(5):558-565.

54. Wigman JT, van Os J, Thiery E, et al. Psychiatric diagnosis revisited: towards a system of staging and profiling combining nomothetic and idiographic parameters of momentary mental states. PloS one. 2013;8(3):e59559.
55. van Os J, Lataster T, Delespaul P, Wichers M, Myin-Germeys I. Evidence that a psychopathology interactome has diagnostic value, predicting clinical needs: an experience sampling study. PloS one. 2014;9(1):e86652.

56. Kramer I, Simons CJ, Wigman JT, et al. Timelagged moment-to-moment interplay between negative affect and paranoia: new insights in the affective pathway to psychosis. Schizophr Bull. 2014; 40(2):278-286.

57. Bird JC, Waite F, Rowsell E, Fergusson EC, Freeman D. Cognitive, affective, and social factors maintaining paranoia in adolescents with mental health problems: A longitudinal study. Psychiatry Res. 2017;257:34-39.

58. Hanssen M, Bak M, Bijl R, Vollebergh W, van Os $\mathrm{J}$. The incidence and outcome of subclinical psychotic experiences in the general population. $\mathrm{Br}$ J Clin Psychol. 2005;44(Pt 2):181-191.

59. Freeman D, Startup H, Dunn G, et al. The interaction of affective with psychotic processes: a test of the effects of worrying on working memory, jumping to conclusions, and anomalies of experience in patients with persecutory delusions. $J$ Psychiatr Res. 2013;47(12):1837-1842.

60. van Rossum I, Dominguez MD, Lieb R, Wittchen $\mathrm{HU}$, van Os J. Affective dysregulation and reality distortion: a 10-year prospective study of their association and clinical relevance. Schizophr Bull. 2011;37(3):561-571.

61. Binbay T, Drukker M, Elbi H, et al. Testing the psychosis continuum: differential impact of genetic and nongenetic risk factors and comorbid psychopathology across the entire spectrum of psychosis. Schizophr Bull. 2012;38(5):992-1002.

62. Myin-Germeys I, van Os J. Stress-reactivity in psychosis: evidence for an affective pathway to psychosis. Clinical psychology review. 2007;27(4):409-424.

63. Morgan C, Reininghaus U, Reichenberg A, et al. Adversity, cannabis use and psychotic experiences: evidence of cumulative and synergistic effects. $\mathrm{Br} J$ Psychiatry. 2014;204(5):346-353.

64. Padmanabhan JL, Shah JL, Tandon N, Keshavan MS. The "polyenviromic risk score": Aggregating environmental risk factors predicts conversion to psychosis in familial high-risk subjects. Schizophr Res. 2017;181:17-22.

65. Cannon TD, Yu C, Addington J, et al. An Individualized Risk Calculator for Research in Prodromal Psychosis. The American journal of psychiatry. 2016;173(10):980-988.

66. Fusar-Poli P, Rutigliano G, Stahl D, et al. Development and Validation of a Clinically Based 
Risk Calculator for the Transdiagnostic Prediction of Psychosis. JAMA psychiatry. 2017;74(5):493500.

67. Gottesman II, Shields J. A polygenic theory of schizophrenia. Vol 58. USA: Proc Natl Acad Sci; 1967.

68. Kaymaz N, Van Os J. Extended psychosis phenotype-yes: single continuum-unlikely: a commentary on 'Why we need more debate on whether psychotic symptoms lie on a continuum with normality'by David (2010). Psychological medicine. 2010;40(12):1963-1966.

69. Linscott RJ, van Os J. Systematic reviews of categorical versus continuum models in psychosis: evidence for discontinuous subpopulations underlying a psychometric continuum. Implications for DSM-V, DSM-VI, and DSM-VII. Annual review of clinical psychology. 2010;6:391-419.

70. Isvoranu AM, Borsboom D, van Os J, Guloksuz S. A Network Approach to Environmental Impact in Psychotic Disorder: Brief Theoretical Framework. Schizophr Bull. 2016;42(4):870-873.
71. Isvoranu AM, van Borkulo CD, Boyette LL, et al. A Network Approach to Psychosis: Pathways Between Childhood Trauma and Psychotic Symptoms. Schizophr Bull. 2017;43(1):187-196.

72. Moffa G, Catone G, Kuipers J, et al. Using Directed Acyclic Graphs in Epidemiological Research in Psychosis: An Analysis of the Role of Bullying in Psychosis. Schizophr Bull. 2017;43(6):1273-1279.

73. European Network of National Networks studying Gene-Environment Interactions in Schizophrenia (EU-GEI). Identifying gene-environment interactions in schizophrenia: contemporary challenges for integrated, large-scale investigations. Schizophrenia Bull. 2014;40(4):729-736.

74. van Os J, Rutten BP, Poulton R. Geneenvironment interactions in schizophrenia: review of epidemiological findings and future directions. Schizophr Bull. 2008;34(6):1066-1082.

75. Guloksuz S, van Os J. The slow death of the concept of schizophrenia and the painful birth of the psychosis spectrum. Psychol Med. 2018;48(2):229-244. 


\begin{tabular}{|c|c|c|c|c|}
\hline & & $\begin{array}{c}\text { T0 } \\
\text { (lifetime) } \\
\mathrm{n}(\%)\end{array}$ & $\begin{array}{c}\text { T1 } \\
\text { (Since T0) } \\
\text { n (\%) }\end{array}$ & $\begin{array}{c}\text { T2 } \\
\text { (Since T1) } \\
\mathrm{n}(\%)\end{array}$ \\
\hline 1. & people were spying on you & $207(3.13)$ & $58(1.09)$ & $43(0.93)$ \\
\hline 2. & people were following you & $126(1.9)$ & $18(0.34)$ & $15(0.32)$ \\
\hline 3. & you were secretly being tested on & $38(0.57)$ & $13(0.25)$ & $9(0.19)$ \\
\hline 4. & someone was conspiring against you & $61(0.92)$ & $12(0.23)$ & $14(0.30)$ \\
\hline 5. & a 'double' had taken the place of a loved one & $2(0.03)$ & $1(0.02)$ & 0 \\
\hline 6. & someone was reading your mind & $49(0.74)$ & $18(0.34)$ & $14(0.3)$ \\
\hline 7. & you could hear the thoughts of others & $53(0.8)$ & $19(0.36)$ & $19(0.41)$ \\
\hline 8. & others could hear your thoughts & $45(0.68)$ & $17(0.32)$ & $8(0.17)$ \\
\hline 9. & alien thoughts were placed in your head & $39(0.59)$ & $16(0.3)$ & $4(0.09)$ \\
\hline 10. & someone took thoughts from your head & $11(0.17)$ & $2(0.04)$ & $1(0.02)$ \\
\hline 11. & special messages were sent to you through media & $25(0.38)$ & $9(0.17)$ & 0 \\
\hline 12. & you were influenced by strange energies & $26(0.39)$ & $8(0.15)$ & $4(0.09)$ \\
\hline 13. & you were being controlled by an outer force & $34(0.52)$ & $6(0.11)$ & $2(0.04)$ \\
\hline 14. & your thoughts were being influenced by machines & $11(0.17)$ & $4(0.08)$ & $3(0.06)$ \\
\hline 15 . & any other delusion reported by subject & $156(2.35)$ & $56(1.06)$ & $32(0.69)$ \\
\hline 16. & you saw things that no one else could see & $198(2.98)$ & $71(1.34)$ & $63(1.36)$ \\
\hline 17. & you could hear things that no one else could hear & $119(1.79)$ & $44(0.83)$ & $32(0.69)$ \\
\hline 18. & your own thoughts were broadcasted & $31(0.47)$ & $14(0.26)$ & $8(0.17)$ \\
\hline 19. & you smelled strange things, that others could not smell & $103(1.55)$ & $56(1.06)$ & $36(0.78)$ \\
\hline 20. & $\begin{array}{l}\text { you had strange sensations, like being touched when no one } \\
\text { was around }\end{array}$ & $149(2.24)$ & $70(1.32)$ & $60(1.3)$ \\
\hline
\end{tabular}

The mean number of psychotic experiences endorsed are $0.22(\mathrm{SD}=0.91), 0.1(\mathrm{SD}=0.57)$, and $0.08(\mathrm{SD}=0.47)$ at $\mathrm{T} 0, \mathrm{~T} 1$, and $\mathrm{T} 2$, respectively. Of those participants who reported any psychotic experience, the majority of individuals endorse either one or two psychotic experiences $(74.59 \%, 78.29 \%$, and 77.08 at T0, T1, and T2, respectively) 




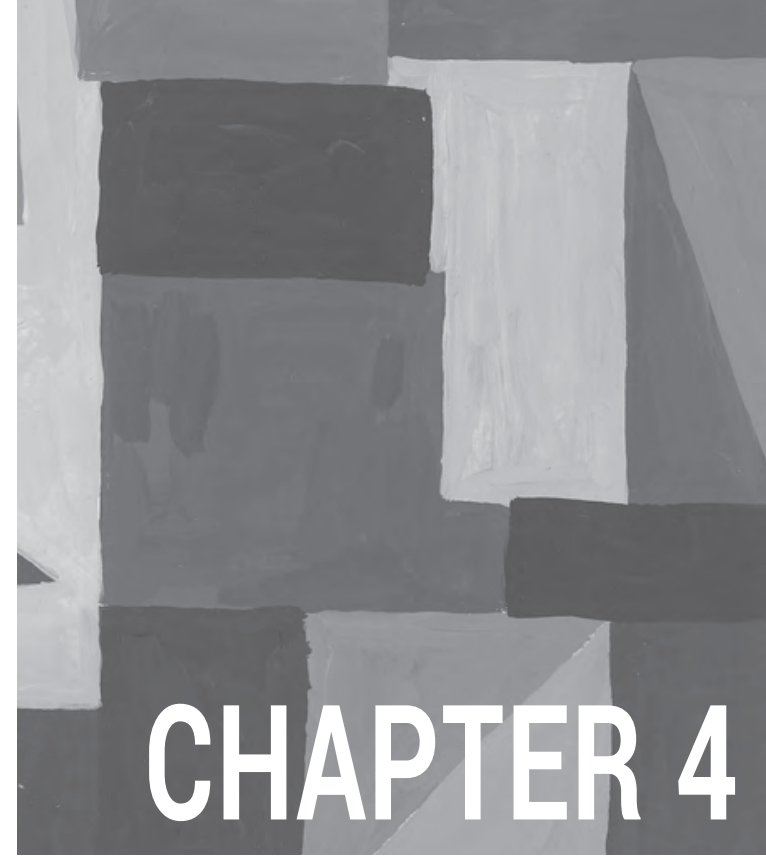

\section{TWINSSCAN - GENE-ENVIRONMENT INTERACTION IN PSYCHOTIC AND DEPRESSIVE INTERMEDIATE PHENOTYPES: RISK AND PROTECTIVE FACTORS IN A GENERAL POPULATION TWIN SAMPLE}

Lotta-Katrin Pries, Clara Snijders, Claudia Menne-Lothmann, Jeroen Decoster, Ruud van Winkel, Dina Collip, Philippe Delespaul, Marc De Hert, Catherine Derom, Evert Thiery, Nele Jacobs, Marieke Wichers, Sinan Guloksuz, Jim van Os, Bart P. F. Rutten

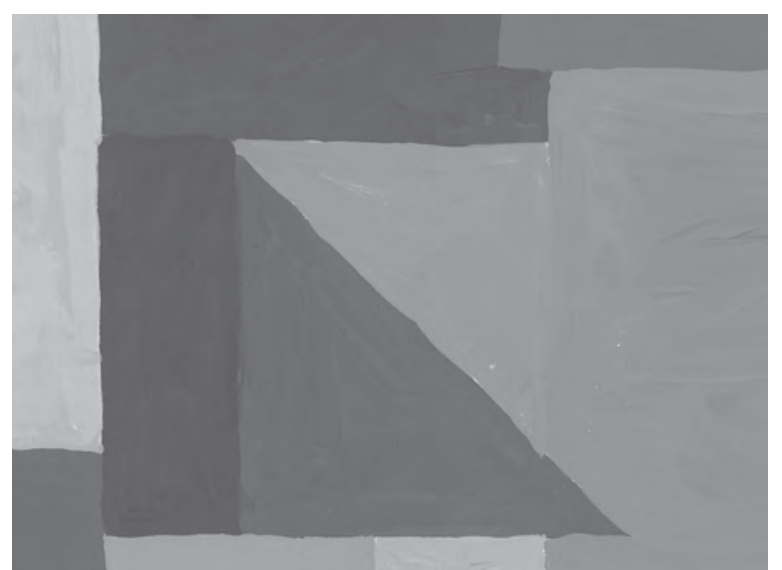




\section{Abstract:}

Meta-analyses suggest that clinical psychopathology is preceded by dimensional behavioral and cognitive phenotypes such as psychotic experiences, executive functioning, working memory, and affective dysregulation that are determined by the interplay between genetic and nongenetic factors contributing to the severity of psychopathology. The liability to mental ill health can be psychometrically measured using experimental paradigms that assess neurocognitive processes such as salience attribution, sensitivity to social defeat, and reward sensitivity. Here, we describe the TwinssCan, a longitudinal general population twin cohort, which comprises 1202 individuals (796 adolescent/young adult twins, 43 siblings, and 363 parents) at baseline. The TwinssCan is part of the European Network of National Networks studying Gene-Environment Interactions in Schizophrenia project and recruited from the East Flanders Prospective Twin Survey. The main objective of this project is to understand psychopathology by evaluating the contribution of genetic and nongenetic factors on subclinical expressions of dimensional phenotypes at a young age, before the onset of disorder, and their association with neurocognitive processes, such as salience attribution, sensitivity to social defeat, and reward sensitivity.

Keywords: Psychosis; depression; environment; general population; genetics; reward sensitivity; salience attribution; social defeat; stress sensitivity; twins. 
The early and prodromal stages of psychopathology are marked with the expression of intermediate phenotypes, including subtle, nonclinical psychotic experiences ${ }^{1-3}$; cognitive impairment ${ }^{4-6}$; and affective dysregulation ${ }^{7,8}$. In the general population, subclinical phenotypes may display low levels of correlation, whereas increased multidimensional psychopathology can be found at the level of psychiatric services ${ }^{9-11}$. Psychotic experiences are more severe in the presence of other dimensional phenotypes such as affective dysregulation ${ }^{12,13}$ and are linked to the severity of psychopathology ${ }^{3,14,15}$. Furthermore, studies show that the genetic and environmental vulnerabilities that are commonly associated with major mental disorders are also nonspecifically associated with different intermediate phenotypes ${ }^{3,11,16-19}$. In addition, greater exposure to genetic and environmental risks drives greater co-occurrence of phenotypes and increased severity of psychopathology ${ }^{3,12}$. Based on this, much attention has been given to study nonclinical multidimensional psychopathology to understand the development of mental ill health.

Several neuropsychological processes have been proposed to index vulnerability and resilience for mental disorders. For example, experiences of subtle alterations in salience attribution in response to environmental information may result in paranoid ideation, hallucinatory experiences and thought interference ${ }^{20,21}$. Similarly, experiences of social defeat are highly stressful for young people and increase the risk for psychopathology ${ }^{22-24}$. Furthermore, stress sensitivity and reward sensitivity, that is, negative affect and positive affect in response to environmental inputs, are crucial mechanisms in both psychotic and nonpsychotic disorders 25-28. On the other hand, experiencing positive emotions such as reward can be protective for psychopathology ${ }^{29,30}$. Therefore, individuals who are particularly skilled in experiencing and seeking such positive experiences can be hypothesized to be less prone to develop a mental disorder than people who are less reward sensitive.

The current cohort was specifically sampled to further evaluate the mechanism of dimensional phenotypes at the early stages of psychopathology. The TwinssCan sample is a unique cohort of a young twin population. It provides deep phenotyping of subclinical symptoms, thorough assessment of putative risk factors (i.e. genetic and nongenetic), and experimental tests to evaluate underlying neuropsychological processes, for example, salience attribution, social defeat, stress sensitivity and reward sensitivity. Therefore, this project is well equipped to investigate not only cross-sectional differences of psychopathology and resilience but also dynamic moment-to-moment variations, as well as long-term trajectories. The primary objective of the project is to investigate risk and protective factors for the development of psychopathology and the role of genetic and nongenetic factors contributing to intermediate phenotypes. Further, it aims to examine whether variations of (1) salience 
attribution, (2) reward sensitivity, (3) stress sensitivity and (4) the level of subtle psychotic experience and affective dysregulation in daily life are associated with genetic vulnerability, exposure to environmental factors, and gene-environment interactions.

\section{Sample Characteristics and Assessments}

Participants were sampled from the East Flanders Prospective Twin Survey (EFPTS), a prospective, population-based registry of multiple births in the province of East Flanders, Belgium ${ }^{31}$. The TwinssCan project recruited across three waves (baseline and two followups). Baseline data were assessed from April 2010 to April $2014^{32}$, including male and female twins in the age range of 15-35 years, their singleton siblings, and their parents. It included 839 participants: 292 monozygotic twins (MZ), 486 dizygotic twins (DZ), 18 triplets and 43 siblings. Furthermore, data from 363 parents were assessed. At baseline, 60\% of the participants were female (MZ: 63\%, DZ: 57\%, triplets: 61\%, siblings: 70\%). The mean age was 17.4 (SD = 3.6) years (MZ: 18.0 [SD = 4.2], DZ: 16.9 [SD = 2.8], triplets: 16.8 [SD = 2.0], and siblings: 20.1 [SD $=4.7]$ ), and most participants had an upper secondary education (primary education: $0.1 \%$, lower secondary education: $6 \%$, upper secondary education: $68 \%$, tertiary education: $26 \%$ ). For the second wave, $60 \%$ of the twins and siblings were reassessed. Sequential analysis based on sex, fetal membranes, umbilical cord blood groups, placental alkaline phosphatase and DNA fingerprints was used to determine zygosity ${ }^{33}$.

Participants were included if they clearly understood and were able to verbally assent to the study procedures and when they voluntarily agreed to participate by means of written informed consent. Signed consent of the parent(s) was required when participants were younger than 18 years. Participation was not possible if caregivers indicated the presence of a pervasive mental disorder. Participants were excluded from the sample if the instructor, study coordinator, or neuropsychological tester confirmed they were not able to complete testing and gave invalid, unreliable data on questionnaires, structured interviews and experimental tests. The local ethics committee approved the study (Commissie Medische Ethiek van de Universitaire ziekenhuizen KU Leuven, Nr. B32220107766).

A broad range of variables were assessed using validated self-report questionnaires, structured interviews and experimental tests. Placenta, blood and saliva samples have been stored at $-80^{\circ} \mathrm{C}$ in biobanks. DNA has been isolated from saliva for all subjects. DNA from placenta and blood is available for the monozygotic twins. A study battery summary of clinical, biological, social-demographics, physical, environmental exposures, cognitive, psychological and experimental measures is reported in Table 1. 
Table 1. TwinssCan Study Battery Summary

\begin{tabular}{|c|c|c|}
\hline & Variable/purpose & Instruments \\
\hline \multirow[t]{9}{*}{ Clinical and Biological } & \multirow{2}{*}{ Positive, negative and depressive symptoms } & Community Assessment of Psychic \\
\hline & & Experiences (CAPE) $)^{* 37}$ \\
\hline & $\begin{array}{l}\text { Somatization, obsessive-compulsive, interpersonal } \\
\text { sensitivity, depression, anxiety, hostility, phobic } \\
\text { anxiety, paranoid ideation, psychoticism }\end{array}$ & $\begin{array}{l}\text { The Symptom Checklist-90-R (SCL-90) } \\
35\end{array}$ \\
\hline & Schizotypy & $\begin{array}{l}\text { Structured Interview for Schizotypy - } \\
\text { Revised (SIS-R) }{ }^{47}\end{array}$ \\
\hline & Depression, psychosis, mania, and PTSD & $\begin{array}{l}\text { Composite International Diagnostic } \\
\text { Interview (CIDI-12) })^{* 56}\end{array}$ \\
\hline & Autism spectrum & Autism-spectrum Quotient (AQ) ${ }^{* 57}$ \\
\hline & Hearing impairment & Short questionnaire \\
\hline & DNA and epigenetic & Blood sample, placenta and cheek swabs \\
\hline & Cortisol & $\begin{array}{l}\text { Salivary cortisol (within the protocol of } \\
\text { the digi-SPEE) }{ }^{45}\end{array}$ \\
\hline
\end{tabular}

\begin{tabular}{|c|c|c|}
\hline Social-demographics & $\begin{array}{l}\text { Date of birth, gender, marital state, housing, } \\
\text { income, job, education, number of siblings, mother } \\
\text { tongue, native country of (grand)parents, age of } \\
\text { parents, education of parents, marital status of } \\
\text { parents, ethnicity, grade of urbanicity, } \\
\text { religion/spirituality }\end{array}$ & The General Demographic Questionnaire \\
\hline \multirow{9}{*}{$\begin{array}{l}\text { Environmental } \\
\text { Exposures }\end{array}$} & Substance-use and alcohol-use & Composite International Diagnostic \\
\hline & & Interview (CIDI-12)* ${ }^{* 6}$ \\
\hline & Cannabis-use & Urine drug screen \\
\hline & Obstetric complications & Obstetric complications \\
\hline & Childhood adversity & $\begin{array}{l}\text { Childhood Trauma Questionnaire - short } \\
\text { form (JTV-SV) }{ }^{58}\end{array}$ \\
\hline & Bullying & $\begin{array}{l}\text { Retrospective Bullying Questionnaire } \\
(\mathrm{RBQ})^{* 59}\end{array}$ \\
\hline & Stressful life events & Life Events Questionnaire ${ }^{60}$ \\
\hline & Differential sibling experiences & $\begin{array}{l}\text { The Sibling Inventory of Differential } \\
\text { Experiences (SIDE) }{ }^{61}\end{array}$ \\
\hline & Self-perception of social rank & Social Comparison Scale (SCS) ${ }^{62}$ \\
\hline Physical & Habitual physical activity & Baecke questionnaire on physical activity ${ }^{63}$ \\
\hline \multirow[t]{8}{*}{$\begin{array}{l}\text { Cognitive and } \\
\text { Psychological }\end{array}$} & Neurotocism and extraversion & $\begin{array}{l}\text { Eysenck Personality Questionnaire (EPQ- } \\
\text { RSS) }^{* 64}\end{array}$ \\
\hline & Prosocial behavior & Prosocial Tendencies Measure (PTM) ${ }^{*} 65$ \\
\hline & Wellbeing & $\begin{array}{l}\text { Amsterdamse Psychological Well Being } \\
\left(\text { AWB }{ }^{* 66}\right.\end{array}$ \\
\hline & Coping & Utrecht Coping list (UCL) $^{\cdot 67}$ \\
\hline & Self-esteem & Rosenberg Self-esteem Scale (RSE) ${ }^{4} 68$ \\
\hline & Positive and negative affect & $\begin{array}{l}\text { Positive and Negative Affect Scales } \\
\text { (PANAS) }{ }^{69} \text { (within the protocol of the } \\
\text { digi-SPEE) }\end{array}$ \\
\hline & Perceived parenting & Parental Bonding Instrument (PBI) ${ }^{70}$ \\
\hline & IQ & $\begin{array}{l}\text { WAIS - Verbal Fluency, Letter Number } \\
\text { Sequencing, Visual Memory Span/Spatial } \\
\text { Span }^{71,72}\end{array}$ \\
\hline \multirow[t]{2}{*}{ Experimental tasks } & Salience attribution & White Noise Test ${ }^{32}$ \\
\hline & Salience attribution & Psycho-babble $^{73}$ \\
\hline
\end{tabular}




\begin{tabular}{lll}
\hline & Variable/purpose & Instruments \\
\hline Experimental tasks & $\begin{array}{l}\text { Daily variations of thoughts, feelings, symptoms, } \\
\text { the current context and appraisal of that context }\end{array}$ & $\begin{array}{l}\text { Experience Sampling Methodology (ESM) } \\
28.38\end{array}$ \\
\cline { 2 - 3 } & Implicit self-esteem & $\begin{array}{l}\text { Single Target - Implicit Association Task } \\
\text { (ST-IAT) for self-esteem }{ }^{74}\end{array}$ \\
\cline { 2 - 3 } & Virtual social defeat & $\begin{array}{l}\text { Digital Social Peer Evaluation Experiment } \\
\text { (digi-SPEE) }\end{array}$ \\
\cline { 2 - 3 } & Reward sensitivity & $\begin{array}{l}\text { Signal Detection Task and reversal with } \\
\text { reward (adapted version) }\end{array}$ \\
\cline { 2 - 3 } & $\begin{array}{l}\text { Positive or negative interpretations of ambiguous } \\
\text { situations }\end{array}$ & $\begin{array}{l}\text { Ambiguous Situations (adapted assessment } \\
\text { part }{ }^{76} \text { ) }\end{array}$ \\
\hline reassessed during wave 2 and wave 3 &
\end{tabular}

\section{Findings}

\section{Genetic and Nongenetic Risk Factors}

Several studies examined how childhood adversity (CA) and genetic liability for psychopathology may affect mental ill health in the TwinssCan cohort. Lecei, et al. ${ }^{34}$ explored whether gene-environment correlation may explain the previously found association between CA and psychopathology. In other words, they tested whether genetic liability for psychopathology made individuals also more likely to experience CA, which would result in a mechanism referred to as genetic confounding of the association between $\mathrm{CA}$ and psychopathology. Within-twin differences of CA were regressed on within-twin differences of psychopathology (assessed with the Symptom Checklist-90-R; SCL-90 ${ }^{35}$ ) in MZ twins. As $M Z$ twins have identical DNA, the researchers argued that associations between withintwin differences indicate that the association between CA and psychopathology cannot be attributed only to genetic predisposition. CA in the whole sample as well as within-twin differences in $\mathrm{CA}$ was associated with psychopathology. These results suggest that at least part of the association between CA and psychopathology is independent from genetic predisposition and therefore genetic confounding cannot explain the association between $\mathrm{CA}$ and psychopathology.

Following this, Pinckaers, et al. ${ }^{36}$ examined whether CA interacted with proxy genetic liability and affected psychopathology in the TwinssCan cohort. Genetic liability was approximated by estimating the co-twins' psychopathology scores on the SCL-90. Genetic vulnerability moderated the association between $\mathrm{CA}$ and the negative dimension of the Community Assessment of Psychic Experiences (CAPE ${ }^{37}$ ). The association with the total CAPE score approached near significance, whereas neither of the other associations, that is, with the subscales positive and depression symptom dimension, showed a significant interaction effect. The results suggest that genetic vulnerability complicated by CA affects subthreshold expression of psychosis, especially in the negative symptom dimension. 
While much attention is given to macro levels of psychopathological changes, which can occur over periods of years or months, recent concepts of psychopathology acknowledge that the development of mental disorders is best understood when also looking at micro, complex, moment-to-moment dynamic changes ${ }^{38}$. Therefore, Pries, et al. ${ }^{39}$ investigated whether molecular genetic risk for schizophrenia interacted with CA and daily life stressors to influence moment-to-moment variations of mental states (i.e., negative affect, positive affect, and subtle psychosis expression) and stress sensitivity. Molecular genetic vulnerability was expressed through polygenic risk score (PRS) for schizophrenia which was calculated by summing weighted trait-alleles ${ }^{40-42}$. Momentary mental states were assessed using a structured diary technique, the experience sampling methodology (ESM) ${ }^{28,38}$. The results were that exposure to early life events showed a statistical interaction with PRS for schizophrenia leading to increased psychosis expressions, negative affect, stress-sensitivity and decreased positive affect. However, daily-life stressors did not significantly interact with genetic markings for the same outcome variables.

In another study on momentary mental states, Vaessen, et al. ${ }^{43}$ used the TwinssCan cohort to evaluate whether sensitivity to daily-life stress predicts onset or persistence of psychopathology. The authors used ESM data at baseline to assess affective responses to daily life stress (i.e., stress sensitivity) and found, contrary to previous work, that stress sensitivity was associated with neither persistence nor onset of psychopathology.

Finally, using the network approach, Hasmi, et al. ${ }^{44}$ investigated whether CA and proxy genetic liability for psychopathology (measured through co-twin scores on the SCL-90-R) were associated with network structures of affective regulation in daily life. The researchers compared regression coefficients, density, and centrality indices of different networks and found that individuals with low genetic liability showed higher overall and negative affect density between network elements, whereas CA was associated with increased positive affect density and overall density.

\section{Experimental Tests}

Three studies used the TwinssCan cohort to validate experimental tasks; the digital social peer evaluation experiment (digi-SPEE) and the white noise test. Menne-Lothmann, et al. ${ }^{45}$ used the digi-SPEE to investigate how negative virtual social evaluation of peers impacts on participants' implicit self-esteem, cortisol levels, and positive and negative affect. For this experimental study, participants were assessed twice. During the first session, baseline assessments were collected. Furthermore, participants were told that they would be coupled with other twins for the next session, based on a rating system. They were instructed to rate 
other participant's profile on intelligence, appearance and congeniality and were told their profile would be rated as well. During the second session, participants were informed that they were rated too low to be allocated to a group, after which follow-up measures were collected. The findings showed that negative affect and cortisol levels were increased after mild negative evaluations, and positive affect as well as self-esteem were reduced. The findings indicate that the digi-SPEE can be used to study important mechanisms of psychopathology and manipulate biological and implicit as well as explicit mental changes.

Following this, Klippel, et al. ${ }^{46}$ evaluated the influence of environmental (i.e., prenatal stress, CA, bullying, and subjective social status) and proxy genetic factors on sensitivity to peer evaluation on the digi-SPEE. Genetic factors and gene-environment interaction did not significantly influence implicit self-esteem, negative affect, and positive affect after negative peer evaluation. However, bullying was associated with increased negative affect and low subjective social status was associated with decreased self-esteem as well as positive affect after peer evaluation.

Pries, et al. ${ }^{32}$ tested whether subclinical expression of psychotic symptoms was associated with experiencing speech illusions that were assessed using the white noise speech illusion task. To detect speech illusions, participants were exposed to white noise and instructed to indicate whether they heard voices and speech fragments. Subtle expressions of psychotic symptoms were measured through the Structured Interview for Schizotypy - Revised (SIS-R ${ }^{47}$ ) and the CAPE. For this purpose, two methodological approaches as published previously in the literature ${ }^{20,21}$, were applied. However, neither method revealed an association between speech illusions and subclinical psychotic expressions in the general population. The findings indicate that contrary to findings in clinical populations, white noise speech illusion may not be associated with psychosis proneness in the adolescent/young adult general population.

\section{Future Directions}

Over the recent years, the TwinssCan project resulted in important findings on the effects of genetic and nongenetic exposures on psychopathology. Additionally, researchers evaluated the applicability of the digi-SPEE task ${ }^{45,46}$ and the white noise speech illusion task ${ }^{32}$ measuring social defeat and salient attribution, respectively. These studies add valuable knowledge that contributes to our current understanding of the complexity of subclinical multidimensional psychopathology, as well as the nonspecific effects of exposures. They highlight the role of gene-environmental interaction focusing both on macro and micro levels of psychopathological changes, that is, changes occurring over month and years ${ }^{34,36,43}$ and from moment to moment ${ }^{39,43,44}$, respectively. 
It is increasingly acknowledged that the development of pleiotropic psychopathology depends on a complex network of environmental exposures, that is, the exposome ${ }^{48,49}$, and polygenic vulnerability ${ }^{50}$, that affect individuals throughout their life. Similarly, resilience is thought to dynamically change and can only be understood by prospectively evaluating different biological and psychological processes $30,51,52$. By benefitting from these recent developments, we recently calculated the exposome score ${ }^{49}$, which we aim to apply to the TwinssCan population. Further, new features are under way. The third wave of the TwinssCan project will soon be processed and provided to the researchers. Future work will include evaluation of epigenetic information in combination with genome-wide molecular data. As the multiples are recruited from the EFPTS, stored placenta samples will be used to compare early life epigenetic variations to markings later in life. As the literature highlights the role of epigenetic variations for psychopathology ${ }^{53,54}$ as well as resilience ${ }^{52,55}$, these approaches are valuable future targets for investigations aiming to better understand factors and processes underlying mental ill health.

\section{Acknowledgments}

The authors thank Jill Ielegems, Katrien Lyssens, Davinia Verhoeven, and Debora op't Eijnde for data-collection. Further, the authors would like to acknowledge that the East Flanders Prospective Twin Survey (EFPTS) is partly supported by the Association for Scientific Research in Multiple Births and that the TwinssCan project is part of the European Community's Seventh Framework Program under grant agreement No. HEALTH-F22009-241909 (Project EU-GEI).

\section{Conflict of interest}

None 


\section{References}

1. Rossler W, Hengartner MP, Ajdacic-Gross V, Haker H, Gamma A, Angst J. Sub-clinical psychosis symptoms in young adults are risk factors for subsequent common mental disorders. Schizophr Res. 2011;131(1-3):18-23.

2. Dominguez MD, Wichers M, Lieb R, Wittchen HU, van Os J. Evidence that onset of clinical psychosis is an outcome of progressively more persistent subclinical psychotic experiences: an 8year cohort study. Schizophr Res. 2009;37(1):8493.

3. Guloksuz S, van Nierop M, Lieb R, van Winkel R, Wittchen HU, van Os J. Evidence that the presence of psychosis in non-psychotic disorder is environment-dependent and mediated by severity of non-psychotic psychopathology. Psychol Med. 2015;45(11):2389-2401.

4. Dominguez MD, Saka MC, Lieb R, Wittchen HU, van Os J. Early expression of negative/disorganized symptoms predicting psychotic experiences and subsequent clinical psychosis: a 10-year study. The American journal of psychiatry. 2010;167(9):10751082.

5. Reichenberg A, Caspi A, Harrington H, et al. Static and dynamic cognitive deficits in childhood preceding adult schizophrenia: a 30-year study. The American journal of psychiatry. 2010;167(2):160169.

6. Ahern E, Semkovska M. Cognitive functioning in the first-episode of major depressive disorder: A systematic review and meta-analysis. Neuropsychology. 2017;31(1):52-72.

7. Fusar-Poli P, Nelson B, Valmaggia L, Yung AR, McGuire PK. Comorbid depressive and anxiety disorders in 509 individuals with an at-risk mental state: impact on psychopathology and transition to psychosis. Schizophr Bull. 2014;40(1):120-131.

8. Häfner H, Maurer K, Trendler G, an der Heiden W, Schmidt M, Könnecke R. Schizophrenia and depression: challenging the paradigm of two separate diseases - a controlled study of schizophrenia, depression and healthy controls. Schizophr Res. 2005;77(1):11-24.

9. Lamers F, van Oppen P, Comijs HC, et al. Comorbidity patterns of anxiety and depressive disorders in a large cohort study: the Netherlands Study of Depression and Anxiety (NESDA). The Journal of clinical psychiatry. 2011;72(3):341-348.

10. Goes FS, McCusker MG, Bienvenu OJ, et al. Comorbid anxiety disorders in bipolar disorder and major depression: familial aggregation and clinical characteristics of co-morbid panic disorder, social phobia, specific phobia and obsessive-compulsive disorder. Psychol Med. 2012;42(7):1449-1459.

11. van Os J, Kenis G, Rutten BP. The environment and schizophrenia. Nature. 2010;468(7321):203212.

12. Pries L-K, Guloksuz S, ten Have M, et al. Evidence That Environmental and Familial Risks for Psychosis Additively Impact a Multidimensional Subthreshold Psychosis Syndrome. Schizophrenia Bull. 2018;44(4):710-719.

13. McGrath JJ, Saha S, Al-Hamzawi A, et al. The Bidirectional Associations Between Psychotic Experiences and DSM-IV Mental Disorders. Am J Psychiat. 2016;173(10):997-1006.

14. Kelleher I, Devlin N, Wigman JT, et al. Psychotic experiences in a mental health clinic sample: implications for suicidality, multimorbidity and functioning. Psychol Med. 2014;44(8):1615-1624.

15. Navarro-Mateu F, Alonso J, Lim CCW, et al. The association between psychotic experiences and disability: results from the WHO World Mental Health Surveys. Acta Psychiat Scand. 2017;136(1):74-84.

16. van Os J, van der Steen Y, Islam MA, et al. Evidence that polygenic risk for psychotic disorder is expressed in the domain of neurodevelopment, emotion regulation and attribution of salience. Psychol Med. 2017;47(14):2421-2437.

17. Nivard MG, Gage SH, Hottenga JJ, et al. Genetic Overlap Between Schizophrenia and Developmental Psychopathology: Longitudinal and Multivariate Polygenic Risk Prediction of Common Psychiatric Traits During Development. Schizophrenia Bull. 2017;43(6):1197-1207.

18. Misiak B, Krefft M, Bielawski T, Moustafa AA, Sasiadek MM, Frydecka D. Toward a unified theory of childhood trauma and psychosis: A comprehensive review of epidemiological, clinical, neuropsychological and biological findings. Neurosci Biobehav Rev. 2017;75:393-406.

19. Brainstorm Consortium, Anttila V, Bulik-Sullivan $\mathrm{B}$, et al. Analysis of shared heritability in common disorders of the brain. Science. 2018;360(6395):eaap8757.

20. Galdos M, Simons C, Fernandez-Rivas A, et al. Affectively salient meaning in random noise: a task sensitive to psychosis liability. Schizophr Bull. 2011;37(6):1179-1186.

21. Catalan A, Simons CJ, Bustamante S, et al. Novel evidence that attributing affectively salient signal to random noise is associated with psychosis. PloS one. 2014;9(7):e102520. 
22. Eisenberger NI, Jarcho JM, Liebermann MD, Naliboff BD. An experimental stduy of shared sensitivity to physical pain and social rejection. Pain. 2006;126:132-138.

23. Nesdaele D, Lambert A. Effects of experimentally manipulated peer rejection on children's negative affect, self-esteem and maladaptive social behavior. International Journal of Behavioral Development. 2007;31(2):115-122.

24. Sandstrom MJ, Cillessen AHN, Eisenhower A. Children's appraisal of peer rejection experiences: Impact on social and emotional adjustment. Soc Dev. 2003;12(4):530-550.

25. Lataster T, Wichers M, Jacobs N, et al. Does reactivity to stress cosegregate with subclinical psychosis? Findings from a general population twin sample. Acta Psychiat Scand. 2009;119(1):45-53.

26. Wichers M, Myin-Germeys I, Jacobs N, et al. Genetic risk of depression and stress-induced negative affect in the flow of daily life. British Jounral of Psychiatry. 2007;191(3):1-6.

27. Myin-Germeys I, van Os J. Stress-reactivity in psychosis: evidence for an affective pathway to psychosis. Clinical psychology review. 2007;27(4):409-424.

28. Delespaul PAEG. Assessing schizophrenia in daily life: The experience sampling method. Maastricht: Maastricht University Press; 1995.

29. Wichers M, Myin-Germeys I, Jacobs N, et al. Evidence that moment-to-moment variation in positive emotions buffer genetic risk for depression: a momentary assessment twin study. Acta Psychiat Scand. 2007;115(6):451-457.

30. Rutten BP, Hammels C, Geschwind N, et al. Resilience in mental health: linking psychological and neurobiological perspectives. Acta Psychiatr Scand. 2013;128(1):3-20.

31. Derom C, Thiery E, Rutten BPF, et al. The East Flanders Prospective Twin Survey (EFPTS): 55 Years Later. Twin Res Hum Genet. 2019:1-6.

32. Pries LK, Guloksuz S, Menne-Lothmann C, et al. White noise speech illusion and psychosis expression: An experimental investigation of psychosis liability. PloS one. 2017;12(8):e0183695.

33. Derom C, Thiery E, Peeters H, Vlietinck R, Defoort P, Frijns JP. The East Flanders Prospective Twin Survey (EFPTS): an actual perception. Twin Res Hum Genet. 2013;16(1):58-63.

34. Lecei A, Decoster J, De Hert M, et al. Evidence that the association of childhood trauma with psychosis and related psychopathology is not explained by gene-environment correlation: A monozygotic twin differences approach. Schizophrenia Research. 2019;205:58-62.
35. Derogatis LR, Rickels K, Rock AF. The SCL-90 and the MMPI: a step in the validation of a new self-report scale. Br J Psychiatry. 1976;128(3):280289.

36. Pinckaers FME, Rotee ILM, Nwosu CV, et al. Evidence for interaction between genetic liability and childhood trauma in the development of psychotic symptoms. Soc Psychiatry Psychiatr Epidemiol. 2019;54(9):1045-1054.

37. Stefanis N, Hanssen M, Smirnis N, et al. Evidence that three dimensions of psychosis have a distribution in the general population. Psychological medicine. 2002;32(2):347-358.

38. van Os J, Verhagen S, Marsman A, et al. The experience sampling method as an mHealth tool to support self-monitoring, self-insight, and personalized health care in clinical practice. Depression anxiety. 2017;34(6):481-493.

39. Pries L-K, Klingenberg B, Menne-Lothmann C, et al. Interaction between polygenic liability for schizophrenia and childhood adversity influences daily-life emotional dysregulation and subtle psychosis expression. 2019.

40. EUGEI investigators. Identifying gene-environment interactions in schizophrenia: contemporary challenges for integrated, large-scale investigations. Schizophr Bull. 2014;40(4):729-736.

41. Ripke S, Neale BM, Corvin A, et al. Biological insights from 108 schizophrenia-associated genetic loci. Nature. 2014;511(7510):421-427.

42. Purcell SM, Wray NR, Stone JL, et al. Common polygenic variation contributes to risk of schizophrenia and bipolar disorder. Nature. 2009;460(7256):748-752.

43. Vaessen T, van Nierop M, Decoster J, et al. Is sensitivity to daily stress predictive of onset or persistence of psychopathology? Eur Psychiat. 2017;45:167-173.

44. Hasmi L, Drukker M, Guloksuz S, et al. Network Approach to Understanding Emotion Dynamics in Relation to Childhood Trauma and Genetic Liability to Psychopathology: Replication of a Prospective Experience Sampling Analysis. Front Psychol. 2017;8:1908.

45. Menne-Lothmann C, Decoster J, van Winkel R, et al. Psychological and Biological Validation of a Novel Digital Social Peer Evaluation Experiment (digi-SPEE). Noro Psikiyatr Ars. 2017;54(1):3-10.

46. Klippel A, Reininghaus U, Viechtbauer W, et al. Sensitivity to Peer Evaluation and Its Genetic and Environmental Determinants: Findings from a Population-Based Twin Study. Child Psychiatry Hum Dev. 2018;49(5):766-778. 
47. Vollema MG, Ormel J. The reliability of the structured interview for schizotypy-revised. Schizophr Bull. 2000;26(3):619-629.

48. Guloksuz S, van Os J, Rutten BPF. The Exposome Paradigm and the Complexities of Environmental Research in Psychiatry. JAMA psychiatry. 2018;75(10):985-986.

49. Pries LK, Lage-Castellanos A, Delespaul P, et al Estimating Exposome Score for Schizophrenia Using Predictive Modeling Approach in Two Independent Samples: The Results from the EUGEI Study. Schizophr Bull. 2019;45(5):960965.

50. Guloksuz S, Pries LK, Delespaul P, et al. Examining the independent and joint effects of molecular genetic liability and environmental exposures in schizophrenia: results from the EUGEI study. World Psychiatry. 2019;18(2):173-182.

51. Kalisch R, Baker DG, Basten U, et al. The resilience framework as a strategy to combat stressrelated disorders. Nature human behaviour. 2017;1(11):784-790.

52. Snijders C, Pries LK, Sgammeglia N, et al. Resilience Against Traumatic Stress: Current Developments and Future Directions. Frontiers in psychiatry. 2018;9:676.

53. Pries L-K, Gülöksüz S, Kenis G. DNA methylation in schizophrenia. In: Neuroepigenomics in Aging and Disease. Springer; 2017:211-236.

54. Rutten BP, Mill J. Epigenetic mediation of environmental influences in major psychotic disorders. Schizophr Bull. 2009;35(6):1045-1056.

55. Rutten BPF, Vermetten E, Vinkers $\mathrm{CH}$, et al. Longitudinal analyses of the DNA methylome in deployed military servicemen identify susceptibility loci for post-traumatic stress disorder. $\mathrm{Mol}$ Psychiatry. 2018;23(5):1145-1156.

56. World Health Organization. The World Health Organization (WHO) Composite International Diagnostic Interview (CIDI). 1990.

57. Hoekstra RA, Bartels M, Cath DC, Boomsma DI. Factor structure, reliability and criterion validity of the Autism-Spectrum Quotient (AQ): a study in Dutch population and patient groups. J Autism Dev Disord. 2008;38(8):1555-1566.

58. Bernstein DP, Stein JA, Newcomb MD, et al. Development and validation of a brief screening version of the Childhood Trauma Questionnaire. Child Abuse Negl. 2003;27(2):169-190.

59. Schäfer M, Korn S, Smith PK, et al. Lonely in the crowd: Recollections of bullying. 2004;22(3):379394.

60. Paykel ES. The Interview for Recent Life Events. Psychol Med. 1997;27(2):301-310.
61. Daniels D, Plomin R. Differential experience of siblings in the same family. Developmental psychology. 1985;21(5):747.

62. Allan S, Gilbert P. A social comparison scale: Psychometric properties and relationship to psychopathology. Personality Individual Differences. 1995;19(3):293-299.

63. Baecke JA, Burema J, Frijters JE. A short questionnaire for the measurement of habitual physical activity in epidemiological studies. Am J Clin Nutr. 1982;36(5):936-942.

64. Sanderman R, Arrindell W, Ranchor A. Het meten van persoonlijkheidskenmerken met de Eysenck Personality Questionnaire (EPQ): een handleiding. Groningen: Noordelijk Centrum voor Gezondheidsvraagstukken. 1995.

65. Carlo G, Randall BA. The development of a measure of prosocial behaviors for late adolescents. Journal of youth and adolescence. 2002;31(1):3144.

66. Ryff CD. Happiness is everything, or is it? Explorations on the meaning of psychological wellbeing. Journal of personality social psychology. 1989;57(6):1069.

67. Schreurs PJG, Willige G, Van De JF, Tellegen B, Graus GMH. De Utrechtse coping lijst: UCL: omgaan met problemen en gebeurtenissen: herziene handleiding 1993. Swets \& Zeitlinger; 1993

68. Rosenberg M. Society and the adolescent selfimage. Princeton, New Jersey: Princeton university press; 1965.

69. Watson D, Clark LA, Tellegen A. Development and validation of brief measures of positive and negative affect: the PANAS scales. J Pers Soc Psychol. 1988;54(6):1063-1070.

70. Parker G, Tupling H, Brown L. A parental bonding instrument. British journal of medical psychology. 1979;52(1):1-10.

71. Krabbendam L, Myin-Germeys I, Hanssen M, van Os J. Familial covariation of the subclinical psychosis phenotype and verbal fluency in the general population. Schizophr Res. 2005;74(1):3741.

72. Wechsler D. Wechsler Memory Scale - 3rd Edition. San Antonio: The Psychological Corporation; 1997.

73. Hoffman RE, Woods SW, Hawkins KA, et al. Extracting spurious messages from noise and risk of schizophrenia-spectrum disorders in a prodromal population. Br J Psychiatry. 2007;191(4):355-356.

74. Rudolph A, Schröder-Abé M, Schütz A, Gregg AP, Sedikides C. Through a glass, less darkly?

Reassessing convergent and discriminant validity in 
measures of implicit self-esteem. European Journal of Psychological Assessment. 2008;24(4):273-281.

75. Pizzagalli DA, Jahn AL, O'Shea JP. Toward an objective characterization of an anhedonic phenotype: a signal-detection approach. Biol Psychiatry. 2005;57(4):319-327.

76. Mathews A, Mackintosh B. Induced emotional interpretation bias and anxiety. J Abnorm Psychol. 2000;109(4):602-615 



\section{Abstract}

Background: An association between white noise speech illusion and psychotic symptoms has been reported in patients and their relatives. This supports the theory that bottom-up and top-down perceptual processes are involved in the mechanisms underlying perceptual abnormalities. However, findings in nonclinical populations have been conflicting.

Objectives: The aim of this study was to examine the association between white noise speech illusion and subclinical expression of psychotic symptoms in a nonclinical sample. Findings were compared to previous results to investigate potential methodology dependent differences.

Methods: In a general population adolescent and young adult twin sample $(n=704)$, the association between white noise speech illusion and subclinical psychotic experiences, using the Structured Interview for Schizotypy-Revised (SIS-R) and the Community Assessment of Psychic Experiences (CAPE), was analyzed using multilevel logistic regression analyses.

Results: Perception of any white noise speech illusion was not associated with either positive or negative schizotypy in the general population twin sample, using the method by Galdos et al. (2011) (positive: ORadjusted: 0.82, 95\% CI: 0.6-1.12, $p=0.217$; negative: ORadjusted: $0.75,95 \%$ CI: 0.56-1.02, $p=0.065)$ and the method by Catalan et al. (2014) (positive: ORadjusted: 1.11, 95\% CI: 0.79-1.57, $p=0.557$ ). No association was found between CAPE scores and speech illusion (OR adjusted: 1.25, 95\% CI: 0.88-1.79, $p=0.220$ ). For the Catalan et al. (2014) but not the Galdos et al. (2011) method, a negative association was apparent between positive schizotypy and speech illusion with positive or negative affective valence (OR adjusted: 0.44, 95\% CI: 0.24-0.81, $p=0.008$ ).

Conclusion: Contrary to findings in clinical populations, white noise speech illusion may not be associated with psychosis proneness in nonclinical populations. 


\section{Introduction}

Epidemiological data indicate that subtle expression of psychosis - in the form of attenuated reality distortion, such as over-valued ideas and perceptual disturbances — is prevalent across the general population, with some predictive value for subsequent mental disorders ${ }^{1,2}$. Recent findings from genetic ${ }^{3}$ and environmental studies of psychosis ${ }^{4,5}$ also suggest etiological continuity across different levels of psychosis manifestation. In this regard, it is reasonable to argue that both subtle psychotic experiences (e.g., perceptual disturbances and rare illusion) and clinically relevant psychotic symptoms (e.g., disturbing hallucinations) may originate from converging neurocognitive processes.

Emerging neuropsychological theories argue that hallucinations might indeed result from an imbalance between top-down (internal factors, e.g., perceptual expectations, prior knowledge, and mental imagery) and bottom-up (i.e., external sensory input) perceptual processing ${ }^{6}$. Top-down processing may have a substantial impact on experiences, for instance, during the identification of degraded or half visible objects. Even more so, during the formation of hallucinations, top-down processing is thought to activate a percept with no external stimulus. In other words, hallucinations are formed when the final percept is disproportionally influenced by internal factors, thus, when top-down processing is prioritized ${ }^{7,8}$.

Previous studies found that exposure to white noise (sound fragments of random noise) induces the perception of speech illusion, presumably by increasing perceptual expectations, thus, operating on top-down processing ${ }^{6,9}$. Several studies observed higher prevalence rates of white noise speech illusion in patients with psychosis and their relatives compared to control cases: around 33\% in patients with psychotic disorders, $14 \%$ in healthy relatives of patients, and $9 \%$ in healthy controls ${ }^{10-12}$. Furthermore, positive psychotic experiences correlated with increased rates of white noise speech illusion in patients and their relatives ${ }^{11}$. Thus, in general, these findings support the notion that the white noise task can be used to understand hallucinations within the framework of top-down processing.

The question rises whether the white noise task can also be used to study perceptual abnormalities in nonclinical samples and whether similar mechanisms (i.e., top-down and bottom-up imbalance) are at play in patients, their relatives, and in healthy controls. Studies using the white noise task to investigate the association between speech illusion and psychotic experiences in the general population are scarce and their findings contradict each other. Several studies found a positive association between subclinical psychotic experiences and white noise speech illusion. Randell, et al. ${ }^{13}$, for instance, investigated white noise speech 
illusion in undergraduate students and found that participants with higher scores on unusual experiences perceived more speech illusion, especially of abstract nature (words like myth, abyss, and sorrow) rather than of concrete nature (words like desk, arm, and letter). Furthermore, Galdos, et al. ${ }^{11}$ found a positive association between positive schizotypy and speech illusion in the white noise task. However, there are also several studies that did not find the above-mentioned association. Using the same measurement tools as Galdos, et al. ${ }^{11}$, Catalan, et al. ${ }^{10}$ observed no association between positive schizotypy and speech illusion in a nonclinical population. Furthermore, in a recent study in children, the experience of hallucinations (last month) and negative affect (last month and lifetime) were only associated with speech illusion in the white noise task when the focus was directed to the affectively salient illusion variable ${ }^{14}$.

Inconsistent results may be partly explained by differences between methodologies, such as measurement tools for psychopathology, sample characteristics, cut-off values for the dichotomous speech illusion variable and analytical strategies ${ }^{10,11,13,14}$. Deeper understanding of underlying mechanisms of perceptual abnormalities in nonclinical populations may foster scientific advances by helping us appraise the dimensional nature and phenomenological features of hallucinations. Therefore, the verification of reliable assessment tools is essential, all the more when the literature is divided. In the current study, we aim to investigate the link between white noise speech illusion and positive subclinical experiences by replicating the various methodologies of preceding studies of the white noise task in the general population ${ }^{10,11}$.

\section{Methods}

Participants were recruited from the East Flanders Prospective Twin Survey (EFPTS; ${ }^{15}$ ), a prospective population-based, multi-birth registry situated in Flanders, Belgium. Zygosity was determined through sequential analysis based on sex, fetal membranes, umbilical cord blood groups, placental alkaline phosphatase, and DNA fingerprints (EFPTS; ${ }^{15}$ ). Individuals who were registered in the EFPTS and who fulfilled the inclusion criteria were invited to participate in the TwinssCan project, a longitudinal study collecting data on adolescents and young adults between the ages of 15 and 35 years, including twins, their siblings, and parents. The TwinssCan project, which started enrollment in April 2010, is a general population based, ongoing longitudinal study ${ }^{16}$ and currently contains information on 1116 participants; twins $(n=830)$, siblings $(n=43)$, and parents $(n=234)$. Participants were included if they clearly understood the study procedure and were able to provide valid, reliable, and complete data. All participants gave written informed consent; for participants below the age of 18, parent(s) also signed an informed consent. Participants were excluded if they had a 
pervasive mental disorder as indicated by caregivers. The local ethics committee (Commissie Medische Ethiek van de Universitaire ziekenhuizen KU Leuven, Nr. B32220107766) approved the study. The current study used a subpopulation of the TwinssCan $(n=704)$ including only monozygotic (MZ) and dizygotic (DZ) twin pairs with complete data on SIS$\mathrm{R}$, the white noise task, age and sex. Table 1 reports the overview of the demographic variables.

Table 1. Summary of selected demographic variables.

\begin{tabular}{|c|c|c|c|}
\hline & $\begin{array}{c}\text { Total } \\
(n=704) \\
\text { mean }(s d)\end{array}$ & $\begin{array}{l}\text { Speech illusion } \\
(\geq 1 ; \mathrm{n}=145) \\
\text { mean }(\mathrm{sd})\end{array}$ & $\begin{array}{c}\text { No speech illusion } \\
(<1 ; \mathrm{n}=559) \\
\text { mean }(\mathrm{sd})\end{array}$ \\
\hline Age in years & $17.3(3.5)$ & $16.9(3.6)$ & $17.4(3.4)$ \\
\hline \multicolumn{4}{|l|}{ Sex } \\
\hline Female & $58.4 \%(\mathrm{n}=411)$ & $51.7 \%(\mathrm{n}=75)$ & $60.1 \%(\mathrm{n}=336)$ \\
\hline Male & $41.6 \%(\mathrm{n}=293)$ & $48.3 \%(\mathrm{n}=70)$ & $39.9 \%(n=223)$ \\
\hline \multicolumn{4}{|l|}{ Zygosity } \\
\hline Monozygotic & $37.4 \%(\mathrm{n}=263)$ & $33.8 \%(\mathrm{n}=49)$ & $38.3 \%(\mathrm{n}=214)$ \\
\hline Dizygotic & $62.6 \%(n=441)$ & $66.2 \%(n=96)$ & $61.7 \%(\mathrm{n}=345)$ \\
\hline \multicolumn{4}{|l|}{ Education level } \\
\hline 0 & $0 \%$ & $0 \%$ & $0 \%$ \\
\hline 1 & $0.1 \%(\mathrm{n}=1)$ & $0 \%$ & $0.2 \%(\mathrm{n}=1)$ \\
\hline 2 & $0 \%$ & $0 \%$ & $0 \%$ \\
\hline 3 & $5.5 \%(\mathrm{n}=39)$ & $4.8 \%(\mathrm{n}=7)$ & $5.7 \%(\mathrm{n}=32)$ \\
\hline 4 & $10.8 \%(\mathrm{n}=76)$ & $13.1 \%(\mathrm{n}=19)$ & $10.2 \%(\mathrm{n}=57)$ \\
\hline 5 & $56.2 \%(\mathrm{n}=396)$ & $56.6 \%(\mathrm{n}=82)$ & $56.2 \%(\mathrm{n}=314)$ \\
\hline 6 & $14.2 \%(\mathrm{n}=100)$ & $14.5 \%(\mathrm{n}=21)$ & $14.1 \%(\mathrm{n}=79)$ \\
\hline 7 & $10.8 \%(\mathrm{n}=76)$ & $5.5 \%(\mathrm{n}=8)$ & $12.2 \%(\mathrm{n}=68)$ \\
\hline Missing & $2.3 \%(\mathrm{n}=16)$ & $5.5 \%(\mathrm{n}=8)$ & $1.4 \%(\mathrm{n}=8)$ \\
\hline \multicolumn{4}{|l|}{ SIS-R } \\
\hline Positive dimension & $0.8(0.4)$ & $0.7(0.3)$ & $0.8(0.4)$ \\
\hline Negative dimension & $0.4(0.2)$ & $0.4(0.2)$ & $0.4(0.2)$ \\
\hline \multicolumn{4}{|l|}{ CAPE } \\
\hline Positive dimension & $1.4(0.3)$ & $1.4(0.3)$ & $1.4(0.3)$ \\
\hline Negative dimension & $1.7(0.4)$ & $1.6(0.3)$ & $1.7(0.4)$ \\
\hline Depressive dimension & $1.8(0.4)$ & $1.7(0.3)$ & $1.8(0.4)$ \\
\hline
\end{tabular}

Speech illusions calculated according to Galdos et al. Education levels range from lower education to university degree; SIS-R: The Structured Interview for Schizotypy—Revised (SIS-R), CAPE: The Community Assessment of Psychic Experiences 


\section{Measurements}

\section{The Structured Interview for Schizotypy—Revised}

The Structured Interview for Schizotypy-Revised (SIS-R) was applied to assess various schizotypy symptoms and signs. The scale has a positive dimension, which includes the symptoms of magical ideation, illusions, referential thinking ( 2 items), psychotic symptoms, and suspiciousness ( 6 items), as well as a negative dimension, which includes the symptoms of introversion, social isolation, restricted affect, and poverty of speech (4 items). In total, both dimensions contain 10 items which are scored on a 4-point Likert scale ranging from absent (0) to severe (3) ${ }^{17}$.

\section{The Community Assessment of Psychic Experiences}

The Community Assessment of Psychic Experiences (CAPE) measures positive and negative psychotic experiences and depressive symptoms in nonclinical samples ${ }^{18}$. The questionnaire consists of 42 items (20 items on positive psychotic experiences, 14 items on negative psychotic experiences, and 8 items on depressive feelings) and has two levels measuring the frequency ( $0=$ never to $4=$ nearly always) and associated distress $(1=$ not distressed to $4=$ very distressed) of the different dimensions. The CAPE shows good reliability and validity in general population samples ${ }^{18,19}$. Data pertaining to the CAPE were collected online.

\section{White noise task}

The white noise task was developed by Galdos, et al. ${ }^{11}$ to assess variations in detecting affectively meaningful speech (speech illusion) in neutral random signals (white noise). While wearing earphones, participants listened to three types of stimuli presented randomly across 75 trials: a) white noise only, b) white noise + clearly audible neutral speech, and c) white noise + barely audible neutral speech. The white noise only condition was the main condition of interest. The latter two conditions with audible speech were added to increase expectation, impacting top-down processing. Participants had to rate the 25 trials in each of the three conditions by pressing one of five buttons: 1 = positive speech illusion (endorsed hearing voice with positive valence), 2 = negative speech illusion (endorsed hearing voice with negative valence), 3 = neutral speech illusion (endorsed hearing voice with neutral valence), $4=$ no speech heard, and $5=$ uncertain (endorsed hearing voice, uncertain affective connotation). The last option aimed to make the rating of 1-3 more conservative. The white noise task was applied with the help of the software E-prime 1.1 (Psychology Software Tools, Pittsburgh, Pennsylvania) and took approximately $15 \mathrm{~min}$. A dichotomized score of "any speech illusion" was calculated, including the occurrence of one or more positive, negative or neutral perceived speech illusion in the 'white noise only' condition ${ }^{11}$. Similar to the study by Catalan, et al. ${ }^{10}$, a second more conservative score was also calculated, which included two or more perceived speech illusion of positive, negative, neutral or uncertain valence. 
Finally, in accordance to the two methods, scores representing "affectively salient speech illusion" (speech illusion with positive or negative valence) were computed.

\section{Statistical analysis}

Individuals from $\mathrm{MZ}$ and $\mathrm{DZ}$ twin pairs, with complete data on SIS-R, the white noise task, age and sex were included in the analyses. Age, sex, and education level were assessed as covariates. As the data have a hierarchical organization, individuals were clustered within twin pairs. Statistical analyses were carried out using the Stata, version $13.1{ }^{20}$ and multilevel logistic regression models were applied using the Stata MEQRLOGIT function.

In an attempt to replicate previous findings as accurately as possible, the methodology of the current study was first construed in line with Galdos, et al. ${ }^{11}$, and subsequently, the data were also analyzed following the methodology of Catalan, et al. ${ }^{10}$.

Conform the description in Galdos, et al. ${ }^{11}$, a more lenient cut-off for any speech illusion ( $\geq 1$ speech illusion with positive, negative or neutral valence) and affective speech illusion ( $\geq 1$ speech illusion with positive or negative valence) was used to create the binary speech illusion variables. Furthermore, in order to construct a 4-level schizotypy variable, in line with the previous study, mean scores of the schizotypy variables (positive and negative) were divided into four categories with equal distant values. For ease of interpretation, these categories were recoded (from 0 to 3). Mixed logistic regression analysis was applied using the white noise speech illusion score (any speech illusion and affective speech illusion in separate analyses) as the dependent variable and the schizotypy variable as the independent variable. The analysis was adjusted for age, sex, and educational level.

Consistent with Catalan, et al. ${ }^{10}$, more conservative binary white noise speech illusion variables were created (any speech illusion: $\geq 2$ speech illusion with positive, negative, neutral or uncertain valence; affective speech illusion: $\geq 2$ speech illusion with positive or negative valence). Binary measures of both dimensions of the positive schizotypy and the CAPE positive scales were calculated by means of median splits. Multilevel logistic regression models were applied using speech illusion scores as the dependent variables and the binary positive schizotypy and CAPE positive variables as the independent variables. The analyses were adjusted for age and sex.

\section{Results}

An overview of selected demographic variables, the total scores of SIS-R and CAPE, and the distribution of white noise speech illusion (according to ${ }^{11}$ ) is provided in the Table 1 . Of the 
704 participants, $145(20.6 \%)$ reported hearing speech in the white noise condition at least once. Education level was missing in 16 participants; therefore, following analyses replicating Galdos, et al. ${ }^{11}$ were conducted in 688 participants. The replication of Galdos, et al. ${ }^{11}$ in this sample yielded no association between any speech illusion (with $\geq 1$ speech illusion) and the 4-level variables of schizotypy subscores: the positive schizotypy scale (ORadjusted: 0.82, 95\% CI: 0.6-1.12, $\mathrm{p}=0.217)$ and the negative schizotypy scale (ORadjusted: 0.75, 95\% CI: $0.56-1.02, \mathrm{p}=0.065)$. The outcome at the different levels of schizotypy is presented in Table 2: In reference to the lowest level (the level 1), only the level 2 showed a negative association with speech illusion. No significant association was found between positive schizotypy and affectively salient speech illusion (positive and/or negative) (OR adjusted: 0.62, 95\% CI: $0.38-1.01, p=0.055)$.

Table 2. White noise speech illusion and the levels of schizotypy.

\begin{tabular}{|c|c|c|c|c|c|}
\hline SIS-R & $\mathrm{N}$ & $\begin{array}{c}\mathrm{n} \text { with any speech } \\
\text { illusion }\end{array}$ & $\begin{array}{c}\text { \% with any speech } \\
\text { illusion }\end{array}$ & $p$-values & $\begin{array}{c}\text { ORadjusted (95\% } \\
\text { CI) }\end{array}$ \\
\hline \multicolumn{6}{|c|}{$\begin{array}{l}\text { Positive } \\
\text { dimension }\end{array}$} \\
\hline Level 1 & 269 & 59 & 43 & -.--- & 1 \\
\hline Level 2 & 369 & 70 & 51 & 0.354 & $0.83(0.6-1.23)$ \\
\hline Level 3 & 48 & 8 & 6 & 0.394 & $0.7(0.31-1.59)$ \\
\hline Level 4 & 2 & 0 & 0 & 0.988 & --.-- \\
\hline \multicolumn{6}{|c|}{$\begin{array}{l}\text { Negative } \\
\text { dimension }\end{array}$} \\
\hline Level 1 & 208 & 52 & 38 & -..-- & 1 \\
\hline Level 2 & 395 & 69 & 50 & 0.018 & $0.61(0.4-0.92)$ \\
\hline Level 3 & 81 & 15 & 11 & 0.185 & $0.65(0.34-1.2)$ \\
\hline Level 4 & 4 & 1 & 1 & 0.976 & -..-.. \\
\hline
\end{tabular}

Using the method by Catalan, et al. ${ }^{10}$, results indicated that 407 participants perceived no speech illusion $(<2)$ and 297 (42.2\%) participants perceived speech illusion $(\geq 2)$. The replication of Catalan, et al. ${ }^{10}$ yielded similar results as the method by Galdos et al. ${ }^{11}$. The variable of any speech illusion was not associated with the positive schizotypy variable (OR adjusted: 1.11, 95\% CI: 0.79-1.57, $p=0.557$ ) or the CAPE positive scale (ORadjusted: 1.25, 95\% CI: $0.88-1.79, p=0.220$ ). There was a significant negative association between the positive schizotypy variable and illusion with affectively salient valence (positive and/or negative; OR adjusted: 0.44, 95\% CI: 0.24-0.81, $p=0.008$ ). 


\section{Discussion}

The current study investigated the association between speech illusion in the white noise task and subtle psychosis expression in the nonclinical population by replicating the methodology by Catalan, et al. ${ }^{10}$ and Galdos, et al. ${ }^{11}$. Around $20.6 \%$ and $42.2 \%$ of the participants reported speech illusion perceptions during the white noise task using the methods of Galdos, et al. ${ }^{11}$ and Catalan, et al. ${ }^{10}$, respectively. Both rates were higher than the $9.0 \%$ and $8.7 \%$ found in previous studies ${ }^{10,11}$. Earlier reports of a positive association between positive schizotypy and the perception of speech illusion could not be replicated in this sample ${ }^{11}$, and the current analyses also revealed no association between the experience of speech illusion and the CAPE scores. Thus, in general, the results are in agreement with Catalan, et al. ${ }^{10}$ showing no significant association between the presence of speech illusion and schizotypy scores in the general population, but contradict the findings of Galdos, et al. ${ }^{11}$ showing a positive association between positive schizotypy and the perception of speech illusion. Using the method by Galdos, et al. ${ }^{11}$, there was a significant association between negative schizotypy and any speech illusion between two of the four levels. However, as the general finding was nonsignificant and the different levels consist of very small samples, this is likely a random finding. Analyses of speech illusion with affective valence indicated that the experience of affectively salient speech illusion was associated with lower positive schizotypy scores; however, this only applied to the method by Catalan, et al. ${ }^{10}$.

An important aspect of the current study was the use of the two analytical methodologies. As Catalan, et al. ${ }^{10}$ already argued, instead of using a median split, statistical power might be increased by splitting participants' schizotypy scores according to the highest and lowest values, similar to the method by Galdos, et al. ${ }^{11}$. Furthermore, Catalan, et al. ${ }^{10}$ and Galdos, et al. ${ }^{11}$ used different cut-off scores and approaches to calculating the dichotomized white noise speech illusion score, which may negatively impact statistical power and speech illusion prevalence rates. Although the present study used both analytical methods, no significant associations between subclinical psychotic symptoms and any speech illusion were observed using either method ${ }^{10,11}$. This supports the notion that there may be no association between speech illusion during the white noise task and subclinical expressions of positive psychotic experiences.

Although several factors might have influenced the current results, one could interpret these results as suggestive that the mechanisms underlying hallucinations in clinical and nonclinical populations are likely different. It was theorized that hallucinations are caused by a top-down and bottom-up imbalance during the perception process. In clinical samples, an alteration of the top-down processes may mediate psychotic symptoms. However, the current findings 
suggest that altered top-down processing of sensory information during the white noise task are unrelated to the expression of subclinical symptoms. Thus, speech illusion in the general population, with the possible exception of children ${ }^{14}$, may not signal a risk to develop a psychotic disorder and may be subject to different mechanisms compared to clinical samples ${ }^{10}$. Previous work suggests that the affective valence is an important factor discriminating auditory hallucinations in healthy controls and patients ${ }^{21,22}$.

Speech illusion with affective connotation were found to display a strong positive association with psychotic disorders ${ }^{14,23}$. However, the current results showed that affective salient speech illusion were negatively associated with positive schizotypy-although this was only apparent using the Catalan, et al. ${ }^{10}$ method. There are several possible explanations for this finding. The first is that it represents a method-related random finding. Another explanation is that the negative association does not necessarily imply that participants with high positive schizotypy scores had fewer illusion with emotional valence; it could also mean that participants with higher scores experienced difficulties in judging the emotional valence of illusion. Previous work indicates that patients with a diagnosis of schizophrenia with auditory-verbal hallucinations, display auditory affect deficits ${ }^{24,25}$. Another explanation is that the association was confounded by cognitive functioning, as this was not controlled for and cognitive functioning has been suggested to impact affect recognition ${ }^{26}$.

Future research may further investigate the significance of cognitive mechanisms, phenomenology, and especially affective valence of speech illusion to better understand the differences between nonclinical and clinical hallucinations. Furthermore, as unique and shared genetic and environmental factors are probably contributing to these differences ${ }^{3,4}$, it may additionally be productive to explore nonclinical hallucinations in relation to genetic and environmental risk factors.

In addition to the theoretical implications, there are several methodological aspects that need to be considered while interpreting the results. In previous studies, attention was mainly directed towards clinical hallucinations, and sample sizes of healthy controls were relatively small. The main strength of the current study is the use of a large general population twin sample. However, splitting the schizotypy scores into four categories, conforming to Galdos, et al. ${ }^{11}$, resulted in subgroups with very few number of participants, particularly higher risk categories. Therefore, this methodology requires an even larger samples size. Another aspect that needs to be considered is the age range of the different studies. Catalan, et al. ${ }^{10}$ argued that their sample included participants with a higher mean age than the sample of Galdos, et al. ${ }^{11}$ and that this could have resulted in differences. This argument was not supported by 
the current study. The sample had a similar mean age than that of Galdos, et al. ${ }^{11}$, but the current study could not find an association between speech illusion and positive psychotic experiences. Furthermore, a recent study in children only showed an association between psychotic experiences and speech illusion when the valence of illusion was considered ${ }^{14}$. Finally, an important difference between the previous and the current study is the ethnicity of the samples. The replicated studies were conducted only in the Spanish population; whereas the current study used participants from the Belgium population. It is possible that cultural and language differences contributed to differential findings.

In summary, the results suggest that, in contrast to clinical samples, white noise speech illusion in nonclinical samples are not associated with the expression of subclinical psychotic experiences ${ }^{10}$. On this basis, it can be speculated that the neuropsychological mechanisms underlying the formation of perceptual abnormalities (speech illusion during white noise task) may be different for psychotic patients and nonclinical general population. Therefore, it may be productive for researchers to evaluate differences in cognitive mechanisms, phenomenology, and affective valence across the spectrum. Finally, the results need to be considered with caution as the samples showed important demographic differences, especially in regard to ethnicity and age. Future studies should replicate the current study with increased attention on the impact of demographic confounders. We plan on replicating this study in an even larger database, the European network of national schizophrenia networks studying gene-environment interaction (EU-GEI, see www.eu-gei.eu), which includes heterogeneous, international, multi-ethnic samples of patients, relatives and healthy controls ${ }^{27,28}$.

\section{Acknowledgements}

The authors thank Jill Ielegems, Katrien Lyssens, Davinia Verhoeven, and Debora op't Eijnde for data-collection and all twins who volunteered for participation. Furthermore, the authors would like to acknowledge that the East Flanders Prospective Twin Survey (EFPTS) is partly supported by the Association for Scientific Research in Multiple Births and that the TwinssCan project is part of the European Community's Seventh Framework Program under grant agreement No. HEALTH-F2-2009-241909 (Project EU-GEI). 


\section{References}

1. Linscott RJ, van Os J. An updated and conservative systematic review and meta-analysis of epidemiological evidence on psychotic experiences in children and adults: on the pathway from proneness to persistence to dimensional expression across mental disorders. Psychol Med. 2013;43(6):1133-1149.

2. van Os J, Linscott RJ, Myin-Germeys I, Delespaul P, Krabbendam L. A systematic review and metaanalysis of the psychosis continuum: evidence for a psychosis proneness-persistence-impairment model of psychotic disorder. Psychol Med. 2009;39(2):179-195.

3. van Os J, van der Steen Y, Islam MA, et al. Evidence that polygenic risk for psychotic disorder is expressed in the domain of neurodevelopment, emotion regulation and attribution of salience. Psychol Med. 2017:1-17.

4. van Os J, Kenis G, Rutten BP. The environment and schizophrenia. Nature. 2010;468(7321):203212.

5. Guloksuz S, van Nierop M, Lieb R, van Winkel R, Wittchen HU, van Os J. Evidence that the presence of psychosis in non-psychotic disorder is environment-dependent and mediated by severity of non-psychotic psychopathology. Psychol Med. 2015;45(11):2389-2401.

6. Aleman A, Bocker KB, Hijman R, de Haan EH, Kahn RS. Cognitive basis of hallucinations in schizophrenia: role of top-down information processing. Schizophr Res. 2003;64(2-3):175-185.

7. de Leede-Smith S, Barkus E. A comprehensive review of auditory verbal hallucinations: lifetime prevalence, correlates and mechanisms in healthy and clinical individuals. Front Hum Neurosci. 2013;7:367.

8. Hugdahl K. "Hearing voices": Auditory hallucinations as failure of top-down control of bottom-up perceptual processes. Scandinavian journal of psychology. 2009;50(6):553-560.

9. Margo A, Hemsley DR, Slade PD. The effects of varying auditory input on schizophrenic hallucinations. Br J Psychiatry. 1981;139(2):122127.

10. Catalan A, Simons CJ, Bustamante S, et al. Novel evidence that attributing affectively salient signal to random noise is associated with psychosis. PloS one. 2014;9(7):e102520.

11. Galdos M, Simons C, Fernandez-Rivas A, et al. Affectively salient meaning in random noise: a task sensitive to psychosis liability. Schizophr Bull. 2011;37(6):1179-1186.

12. Vercammen A, de Haan EH, Aleman A. Hearing a voice in the noise: auditory hallucinations and speech perception. Psychol Med. 2008;38(8):11771184.

13. Randell J, Goyal M, Saunders J, Reed P. Effect of a context of concrete and abstract words on hallucinatory content in individuals scoring high in schizotypy. J Behav Ther Exp Psychiatry. 2011;42(2):149-153.

14. Rimvall MK, Clemmensen L, Munkholm A, et al. Introducing the White Noise task in childhood: associations between speech illusions and psychosis vulnerability. Psychol Med. 2016;46(13):27312740 .

15. Derom C, Thiery E, Peeters H, Vlietinck R, Defoort P, Frijns JP. The East Flanders Prospective Twin Survey (EFPTS): an actual perception. Twin Res Hum Genet. 2013;16(1):58-63.

16. Menne-Lothmann C, Decoster J, van Winkel R, et al. Psychological and biological validation of a novel digital experimental social peer evaluation exposure (digi-SPEE). Archives of Neuropsychiatry. 2016: Forthcoming.

17. Vollema MG, Ormel J. The reliability of the structured interview for schizotypy-revised. Schizophr Bull. 2000;26(3):619-629.

18. Stefanis N, Hanssen M, Smirnis N, et al. Evidence that three dimensions of psychosis have a distribution in the general population. Psychological medicine. 2002;32(2):347-358.

19. Konings M, Bak M, Hanssen M, van Os J, Krabbendam L. Validity and reliability of the CAPE: a self-report instrument for the measurement of psychotic experiences in the general population. Acta Psychiatr Scand. 2006;114(1):55-61.

20. StataCorp. Release 13. Statistical software. StataCorp LP, College Station, TX. 2013.

21. Honig A, Romme MAJ, Ensink BJ, Escher SDMAC, Pennings MHA, Devries MW. Auditory hallucinations: A comparison between patients and nonpatients. Journal of Nervous and Mental Disease. 1998;186(10):646-651.

22. Choong C, Hunter MD, Woodruff PWR. Auditory hallucinations in those populations that do not suffer from schizophrenia. Current Psychiatry Reports. 2007;9(3):206-212.

23. Daalman K, Boks MP, Diederen KM, et al. The same or different? A phenomenological comparison of auditory verbal hallucinations in healthy and 
psychotic individuals. J Clin Psychiatry.

2011;72(3):320-325

24. Abbott G, Byrne LK. Schizotypal traits are associated with poorer identification of emotions from dynamic stimuli. Psychiatry Res. 2013;207(12):40-44.

25. Rossell SL, Boundy CL. Are auditory-verbal hallucinations associated with auditory affective processing deficits? Schizophr Res. 2005;78(1):95106.

26. Kohler CG, Bilker W, Hagendoorn M, Gur RE, Gur RC. Emotion recognition deficit in schizophrenia: association with symptomatology and cognition. Biological psychiatry.

2000;48(2):127-136.

27. van Os J, Rutten BP, Poulton R. Geneenvironment interactions in schizophrenia: review of epidemiological findings and future directions. Schizophr Bull. 2008;34(6):1066-1082.

28. European Network of National Networks studying Gene-Environment Interactions in Schizophrenia. Identifying gene-environment interactions in schizophrenia: contemporary challenges for integrated, large-scale investigations. Schizophr Bull. 2014;40(4):729-736. 



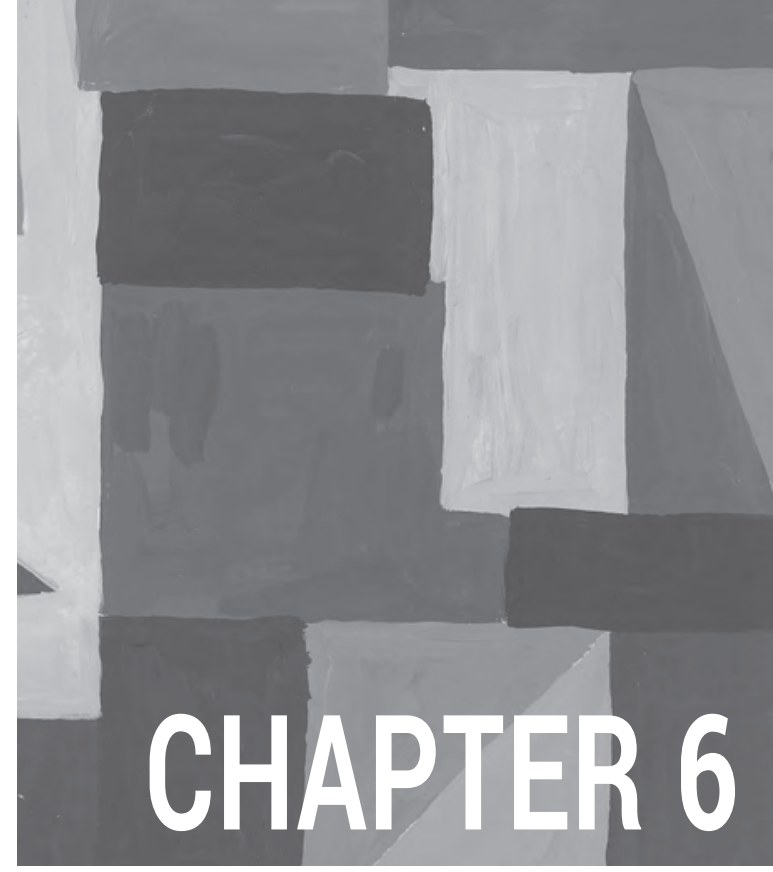

\section{POLYGENIC LIABILITY FOR SCHIZOPHRENIA AND CHILDHOOD ADVERSITY INFLUENCES DAILY- LIFE EMOTION DYSREGULATION AND PSYCHOSIS PRONENESS}

Lotta-Katrin Pries, Boris Klingenberg, Claudia Menne-Lothmann, Jeroen Decoster, Ruud van Winkel, Dina Collip, Philippe Delespaul, Marc De Hert, Catherine Derom, Evert Thiery, Nele Jacobs, Marieke Wichers, Ozan Cinar, Bochao D. Lin, Jurjen J. Luykx, Bart P. F. Rutten, Jim van Os, Sinan Guloksuz

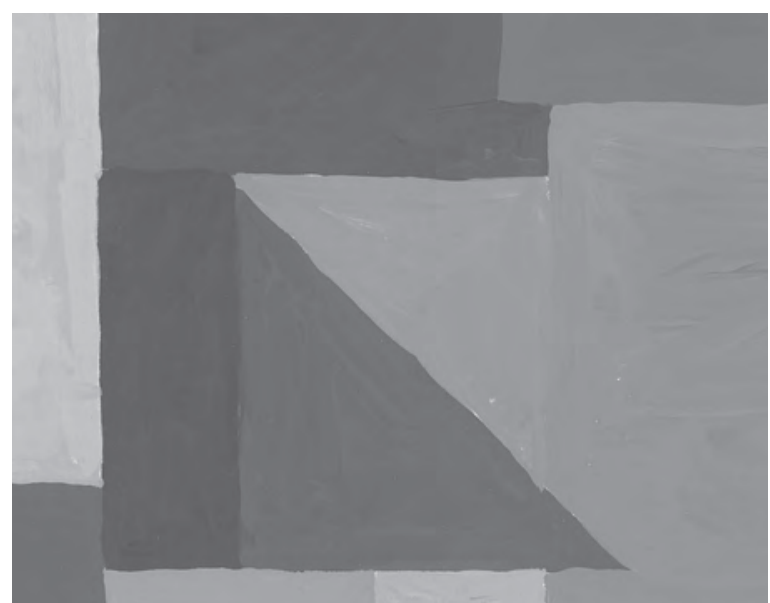




\section{Abstract}

Objective: To test whether polygenic risk score for schizophrenia (PRS-S) interacts with childhood adversity and daily-life stressors to influence momentary mental state domains (negative affect, positive affect, and subtle psychosis expression) and stress-sensitivity measures.

Methods: The data were retrieved from a general population twin cohort including 593 adolescents and young adults. Childhood adversity was assessed using the Childhood Trauma Questionnaire. Daily-life stressors and momentary mental state domains were measured using ecological momentary assessment. PRS-S was trained on the latest Psychiatric Genetics Consortium schizophrenia meta-analysis. The analyses were conducted using multilevel mixed-effects tobit regression models.

Results: Both childhood adversity and daily-life stressors were associated with increased negative affect, decreased positive affect, and increased subtle psychosis expression, while PRS-S was only associated with increased positive affect. No gene-environment correlation was detected. There is novel evidence for interaction effects between PRS-S and childhood adversity to influence momentary mental states [negative affect $(\mathrm{b}=0.07, P=0.013)$, positive affect $(\mathrm{b}=-0.05, P=0.043)$, and subtle psychosis expression $(\mathrm{b}=0.11, P=0.007)]$ and stress-sensitivity measures.

Conclusion: Exposure to childhood adversities, particularly in individuals with high PRS-S, is pleiotropically associated with emotion dysregulation and psychosis proneness.

\section{Keywords}

gene-environment interaction; psychosis; childhood trauma; daily-life stressors; experience sampling method; emotion regulation 


\section{Significant outcomes}

- Early-life stressors and daily-life stressors influence momentary mental state domains (negative affect, positive affect, and subtle psychosis expression).

- Polygenic risk for schizophrenia moderates the association between early-life stressors and momentary mental state domains as well as stress-sensitivity measures.

- It appears that the exposure to childhood adversities, especially in individuals with high molecular genetic risk for schizophrenia, is linked to emotion dysregulation and psychosis proneness.

\section{Limitations}

- Daily-life stressors may not only influence momentary mental states but also be influenced by them.

- Measuring psychosis proneness in the general population may be more challenging than measuring emotional reactivity.

\section{Introduction}

Converging evidence suggests that the genetic and nongenetic vulnerability contributing to the development of schizophrenia and related psychotic disorders is shared across a broad range of psychotic and non-psychotic clinical syndromes and expressed non-specifically in the affective, psychotic, and cognitive domains in the general population ${ }^{1-5}$. Understanding the pleiotropic effects of risk factors associated with schizophrenia on the earliest stages of pluripotent psychopathology may therefore pave the way for gaining insight into the shared biological and mental processes underlying psychosis spectrum disorder (PSD). Contemporary concepts of mental disorders acknowledge psychopathology as a highly dynamic and time-varying complex system that can only be understood from its interconnected constituent parts. These concepts provide a useful theoretical framework to investigate how alterations of micro-level transdiagnostic mental states, varying from moment-to-moment, precede the transition to the more discrete clinical syndrome of PSD ${ }^{6}$.

Studies of ecological momentary assessment (EMA), designed to collect micro-level mental state variation, have consistently shown that disturbed emotional (affective dysregulation) and psychotic reactivity to daily-life stressors (aberrant salience attribution) are associated with psychosis expression in different populations at varying severity stages, the general population, clinical high-risk samples, siblings of patients with PSD, and cases ${ }^{7-13}$. Further, in agreement with the diathesis-stress theory ${ }^{14}$, EMA studies have provided evidence that genetic and environmental vulnerabilities are associated with alterations in emotional 
reactivity. Individuals who experienced childhood adversity (CA) showed heightened emotional and psychotic reactivity to daily-life stressors ${ }^{15-19}$, and increased persistence of momentary mental states ${ }^{20}$; the influence of CA on the reactivity to daily-life stressors was stronger in populations with increased proxy genetic risk (i.e. service users, clinical high risk, or first-episode psychosis compared to healthy controls) $)^{15,16}$.

Early hypothesis-driven candidate gene studies also provided some evidence for the role of gene-environment interaction $(\mathrm{G} \times \mathrm{E})$ in affective and psychotic reactivity to daily-life stressors $^{21-23}$. While these studies can be considered the first steps in understanding the genetic correlates of daily stress-reactivity, they were undersized and by design too simplistic to capture the complex genetic architecture. The use of cumulative risk scores-polygenic risk score (PRS) — as a single molecular metric has significantly enhanced the power to detect $\mathrm{G} \times \mathrm{E}$ without compromising the validity of the results ${ }^{24}$. We previously showed that the likelihood of schizophrenia is increased as a function of the interaction between PRS for schizophrenia (PRS-S) and childhood adversities as well as cannabis use ${ }^{25}$.

A recent perspective article discusses how real-time measurement of cognitive and emotional processes via EMA, which eliminates retrospective recall bias, combined with modern polygenic approach may greatly advance our understanding of the role of $\mathrm{G} \times \mathrm{E}$ in psychopathology and mental wellbeing ${ }^{26}$. PRS-based approaches for testing $\mathrm{G} \times \mathrm{E}$ represent a novel approach, and to the best of our knowledge, no EMA study has utilized PRS-S yet.

In this study, guided by the transdiagnostic Research Domain Criteria (RDoC) framework prioritizing shared dimensional psychological constructs cutting across diagnostic categories $^{27}$, we outlined a step-by-step analytical plan to test the contribution of $\mathrm{G} \times \mathrm{E}$ to altered emotional processes, previously associated with the earliest stages of PSD. Bringing together a unique sampling frame of a general population twin cohort of young adults and adolescents with rich EMA data, we aimed to investigate for the first time whether molecular genetic risk score for schizophrenia (PRS-S) interacts with early-life stressors (CA) and dailylife stressors (social, event, activity, and overall stress) to influence momentary mental state domains (negative affect, positive affect, and subtle psychosis expression), and whether PRS$\mathrm{S}$ moderates the association between CA and stress-sensitivity measures.

\section{Methods}

\section{Sample}

Data were derived from the first wave of the TwinssCan, a general population twin cohort that started including adolescent and young adult (age range $=15-35$ years) twins $(\mathrm{n}=796)$, 
their siblings $(\mathrm{n}=43)$, and parents $(\mathrm{n}=363)$ from April 2010 to April 201428. The TwinssCan cohort comprises individuals fulfilling the inclusion criteria from the East Flanders Prospective Twin Survey ${ }^{29}$, a prospective population-based, multi-birth registry positioned in Flanders, Belgium. Participants were excluded if they had a pervasive mental disorder as indicated by caregivers. All participants gave written informed consent and parent(s) signed an informed consent for participants below the age of 18 years. The local ethics committee approved the study (Commissie Medische Ethiek van de Universitaire ziekenhuizen KU Leuven, Nr. B32220107766). Sequential analysis based on sex, fetal membranes, umbilical cord blood groups, placental alkaline phosphatase, and DNA fingerprints was used to determine zygosity ${ }^{29}$.

\section{Measures}

\section{Ecological Momentary Assessment (EMA)}

EMA is a well-validated structured diary technique that assesses individual and contextual measures in the current moment, throughout the day ${ }^{30-32}$. During the assessment period (six consecutive days), participants used a digital device (PsyMate) $)^{33}$ to electronically fill out a brief questionnaire assessing their emotions, thoughts, context and their appraisal of that context 10 times/day at an unpredictable moment (semi-random) in each of ten 90-min time blocks between 7:30 and 22:3033.

Conforming to previously described methods, the negative affect (NA) ${ }^{15}$ and the positive affect $(\mathrm{PA})^{34}$ domains were the mean scores of items assessing emotional states. Subtle psychosis expression (PE) was the mean score of items concerning psychotic-like experiences $^{35}$. Daily-life stress domains were constructed as event ${ }^{36}$, social ${ }^{15}$, activity ${ }^{15}$, and overall stress (average of event, social, and activity stress). For detailed description of EMA items, see Table 1.

\section{Childhood adversity}

CA was assessed using the Childhood Trauma Questionnaire (CTQ) ${ }^{37}$ that consists of 28 items rated on a 5-point Likert scale assessing five domains of maltreatment (emotional and physical neglect along with emotional, physical, and sexual abuse). Table S3 reports the frequencies of childhood adversity domains. Consistent with previous work ${ }^{38}$ using this dataset, CA was defined as the mean score of all five domains.

\section{Genotyping, imputation, and PRS}

Genotypes of the twins and their siblings were generated on two platforms: the Infinium CoreExome-24 and Infinium PsychArray-24 kits. Quality control (QC) procedures were 
performed using PLINK v1.9 $9^{39}$ in both datasets separately. Single nucleotide polymorphisms (SNPs) and participants with call rates below 95\% and 98\%, respectively, were removed. A strict SNP QC only for subsequent sample QC steps was then conducted. This involved a minor allele frequency (MAF) threshold $>10 \%$ and a Hardy-Weinberg equilibrium (HWE) $P$-value $>10^{-5}$, followed by linkage disequilibrium (LD)-based SNP pruning $\left(\mathrm{R}^{2}<0.5\right)$. This resulted in $-58 \mathrm{~K}$ SNPs to assess sex errors $(\mathrm{n}=8)$, heterozygosity $[\mathrm{F}<5 \mathrm{x}$ the standard deviation $(\mathrm{SD}), \mathrm{n}=3$ ], homozygosity $(\mathrm{F}>5 \mathrm{x} \mathrm{SD})$, and relatedness by pairwise identity by descent (IBD) values (monozygotic: $\hat{p}<0.9$, dizygotic and full siblings: $\hat{p}>0.65$ or $\hat{p}>0.35, \mathrm{n}=5$ ). The ancestry-informed principal components (PCs) analyses were conducted by EIGENSTRAT ${ }^{40}$. The ethnic outliers of which the first 4 PCs diverged > 10x SD from Utah residents with Northern and Western European ancestry from the CEPH collection (CEU) and Toscani in Italia (TSI) samples $(\mathrm{n}=5)$, and $>3 \mathrm{x}$ SD of the TwinssCan samples $(\mathrm{n}=7)$ were excluded. After removing these subjects, a regular SNP QC was performed (SNP call rate $>98 \%$, HWE $\mathrm{p}>1 \mathrm{e}-06, \mathrm{MAF}>1 \%$, and strand ambiguous SNPs and duplicate SNPs were removed).

The two QCed datasets were imputed on the Michigan server ${ }^{41}$ using the HRC r1.1 2016 reference panel with European samples after phasing with Eagle v2.3. Post-imputation QC involved removing SNPs with imputation quality $\left(\mathrm{R}^{2}\right)<0.8$, with a MAF $<0.01$, SNPs that had a discordant MAF compared to the reference panel (MAF difference with HRC reference $>0.15$ ), as well as strand ambiguous AT/CG SNPs and multi-allelic SNPs. The two chips were merged and an additional check for MAF $>0.01$, HWE $P>1$ e-06 was executed, which resulted in 3,407,392 SNPs for 688 individuals.

PRS-S were calculated based on the meta-analysis results from the Psychiatric Genetics Consortium (PGC)-2 SZ and the CLOZUK sample (schizophrenia cases on clozapine from the $\mathrm{UK})^{42}$. Then insertions and deletions, ambiguous SNPs, SNPs with a MAF < 0.01 , imputation quality $\mathrm{R}^{2}<0.9$, SNPs located in complex-LD regions and long-range LD regions ${ }^{43}$ were excluded. Overlapping SNPs between the schizophrenia GWAS (training), 1000 genomes (reference), and our dataset (target) were selected. These SNPs were clumped in two rounds using PLINK's clump function (round 1: --clump-kb 250 --clumpr2 0.5; round 2: --clump-kb 5000 --clump-r2 0.2), resulting in 88,736 SNPs for PRS-S calculation. Odds ratios for autosomal SNPs reported in the schizophrenia summary statistics were log-converted into beta values. PRS-S were calculated using PLINK's score function. Informed by the PGC analyses, PRS-S with cut-off $P<0.05$ (including 21,901 SNPs) was used in the following analyses to achieve a balance between the number of falsepositive and true-positive risk alleles ${ }^{44}$. For details, see supplementary information. 
Table 1 Description of EMA variables

Mean score of 5 items (I feel anxious, lonely, down, insecure and irritated)

Negative affect $\quad$ Each item was rated on a 7-point Likert scale ranging from 1 (not at all) to

7 (very).

\begin{tabular}{|c|c|}
\hline Positive affect & $\begin{array}{l}\text { Mean score of } 4 \text { items (I feel cheerful, satisfied, relaxed, and globally } \\
\text { feeling well). Each item was rated on a 7-point Likert scale ranging from } 1 \\
\text { (not at all) to } 7 \text { (very). }\end{array}$ \\
\hline Subtle psychosis expression & $\begin{array}{l}\text { Mean score of } 5 \text { items (suspiciousness, being afraid of losing control, racing } \\
\text { thoughts, pervasive thoughts, and difficulties to express thoughts). Each } \\
\text { item was rated on a 7-point Likert scale ranging from } 1 \text { (not at all) to } 7 \\
\text { (very). }\end{array}$ \\
\hline Event stress & $\begin{array}{l}\text { Participants were asked to rate the most important event since last entry on } \\
\text { its pleasantness on a bipolar Likert scale from }-3 \text { (very unpleasant) to } 3 \\
\text { (very pleasant). Consistent with previous studies }{ }^{36} \text {, ratings from }-3 \text { to }-1 \\
\text { were considered stressful and scores from } 1 \text { to } 3 \text { were recoded to } 0 \text {. For } \\
\text { simplification, the score was reversed and very unpleasant events therefore } \\
\text { represented the highest score on a scale from } 0-3 \text {. }\end{array}$ \\
\hline Social stress & $\begin{array}{l}\text { Participants were asked with whom they currently were (e.g., nobody or } \\
\text { family). When participants reported to be alone, they were asked to answer } \\
\text { the following items: I like to be alone (reversed); I would prefer to have } \\
\text { company; and I feel safe alone (reversed). When participants reported to be } \\
\text { in company, they were asked the following items: I would prefer to be } \\
\text { alone; I find the people I am with pleasant (reversed); I feel safe (reversed); } \\
\text { I feel I belong (reversed); and I feel judged. All items were scored on a } \\
\text { Likert scale from } 1 \text { (not at all) to } 7 \text { (very), and the mean score of all items } \\
\text { was used to estimate the social stress. }\end{array}$ \\
\hline Activity stress & $\begin{array}{l}\text { Participants were asked about the activity they participated in just before } \\
\text { the beep (e.g., resting, watching TV, and smoking). The mean score of the } \\
\text { following items [rated on a 7-point Likert scale from } 1 \text { (not at all) to } 7 \\
\text { (very)] was used to calculate an activity stress score: I would prefer doing } \\
\text { something else; This activity is difficult for me; and I can do this well } \\
\text { (reversed). }\end{array}$ \\
\hline Overall stress & Average of event-, social-, activity stress. \\
\hline
\end{tabular}

EMA: Ecological momentary assessment

\section{Statistical analyses}

For the purpose of this analysis, parents were excluded. Only participants with complete data on the CTQ, age, sex, and PRS-S were included in the analyses. Conforming to previous studies $^{32}$, participants who completed less than 1/3 of the EMA questionnaires were excluded from the analysis $(n=52)$. One individual with a visibly extreme value of CA ( 7 SD from the mean) was excluded from analyses. The final sample included 593 participants: monozygotic $(n=180)$, dizygotic $(n=380)$ twin pairs, and their siblings $(n=33)$. 
The data have a hierarchical structure. Multiple EMA observations (level 1) were clustered within subjects (level 2), who were part of twin pairs (level 3). Multilevel mixed-effects model is the recommended method to handle data including observations at more than one level in terms of unit of analysis by taking into account of the variability associated with each level of nesting ${ }^{45-47}$. To handle this nested structure including familial relatedness, multilevel mixedeffects models were applied. In the current study, as typically observed in EMA studies, left censoring (NA or PE) and right censoring (PA) were present due to a greater amount of observations with a score of one (NA or PE) or seven (PA) on the outcome variables. In consideration of the skewness, multilevel mixed tobit regression ${ }^{48}$ (censored regression) with an unstructured covariance matrix was performed using the Stata version $15.0^{49}$ "METOBIT" command. The independent variables [PRS-S, CA, and daily-life stressors (overall, event, social, and activity stress)] were standardized and centered $(\min =0, \mathrm{SD}=1)$.

First, we analyzed associations of CA and PRS-S, and their interaction, with EMA outcomes. Second, we tested associations of daily-life stressors, and their interaction with PRS-S, and EMA outcomes. Third, for sensitivity analyses, we constructed stress-sensitivity measures for use in $\mathrm{G} \times \mathrm{E}$ analyses. Consistent with previous work $^{50}$, we applied a two-step approach to analyze stress-sensitivity. First, separate multilevel tobit regression models including the daily-life stressors as independent variables and EMA outcomes as dependent variables were estimated. The models were fitted via maximum likelihood and included a random slope for the daily-life stressor. From these models, fitted (predicted) values (substituting maximum likelihood estimates for fixed effects and empirical Bayes predictions for random effects) were stored as stress-sensitivity (e.g. NA-Event stress-sensitivity; the association between event stress and NA) scores. Eventually, we tested associations between CA and PRS-S, and their interaction, with normally distributed stress-sensitivity scores as dependent variables in multilevel linear regression models using the "MIXED" command.

All models were controlled for a priori covariates (age and sex), while models including PRS$S$ were additionally adjusted for ancestry, using the first 2 genomic principal components (PCs). To adequately control for confounding ${ }^{51}$, interaction models included these covariates not only as main effects but also covariate $\mathrm{x}$ environment and covariate $\mathrm{x}$ PRS-S interaction terms.

\section{Results:}

Sample characteristics are reported in Table 2. A correlation matrix of the three momentary mental state domains is provided in Table S4. 


\section{Main associations and interactions of CA and PRS-S on momentary mental state domains}

CA was associated with increased NA, decreased PA, and increased PE, while PRS-S was only associated with increased PA (Table 3). These results remained significant after controlling for daily-life stressors (Table S5). No gene-environment correlation was present as PRS-S was not associated strongly or significantly with $\mathrm{CA}(\mathrm{b}=-0.01,95 \% \mathrm{CI}=-0.03$ to $0.02, P=$ 0.676).

PRS-S moderated the association of CA with all three momentary mental state domains, while only NA and PE reached the Bonferroni-adjusted statistical significance level (Table 3). The interaction effects remained significant after controlling for daily-life stressors (Table S5). As shown in Figure 1, visualizing the fitted interaction effects between PRS-S and CA on momentary mental state domains, the association between CA and mental state domains increased as a function of increased PRS-S (for scatter plots of raw data, see Figure S3).

Table 2 Sample characteristics

\begin{tabular}{lc}
\hline & $\begin{array}{c}\text { Total sample }(\mathrm{N}=593) \\
\text { Mean (SD), EMA observations }\end{array}$ \\
\hline Sex & $362(61 \%)$ \\
Female & $231(39 \%)$ \\
Male & $17.60(3.81)$ \\
Age & $1.35(0.31)$ \\
Childhood adversity & $1.78(0.84), \mathrm{n}=23293^{\dagger}$ \\
Negative affect & $5.06(1.06), \mathrm{n}=23265^{\dagger}$ \\
Positive affect & $1.89(0.99), \mathrm{n}=23272^{\dagger}$ \\
Subtle psychosis expression & $1.89(0.78), \mathrm{n}=23225^{\dagger}$ \\
Overall stress & $0.34(0.84), \mathrm{n}=22812^{\dagger}$ \\
Event stress & $2.33(1.08), \mathrm{n}=23172{ }^{\dagger}$ \\
Social stress & $2.95(1.43), \mathrm{n}=23192^{\dagger}$ \\
Activity stress & \\
\hline
\end{tabular}

${ }^{\dagger}$ Number of observations, EMA: Ecological momentary assessment, PRS-S: Polygenic risk score for schizophrenia, SD: Standard deviation 
Figure 1 Interaction effect of CA and PRS-S on momentary mental state domains
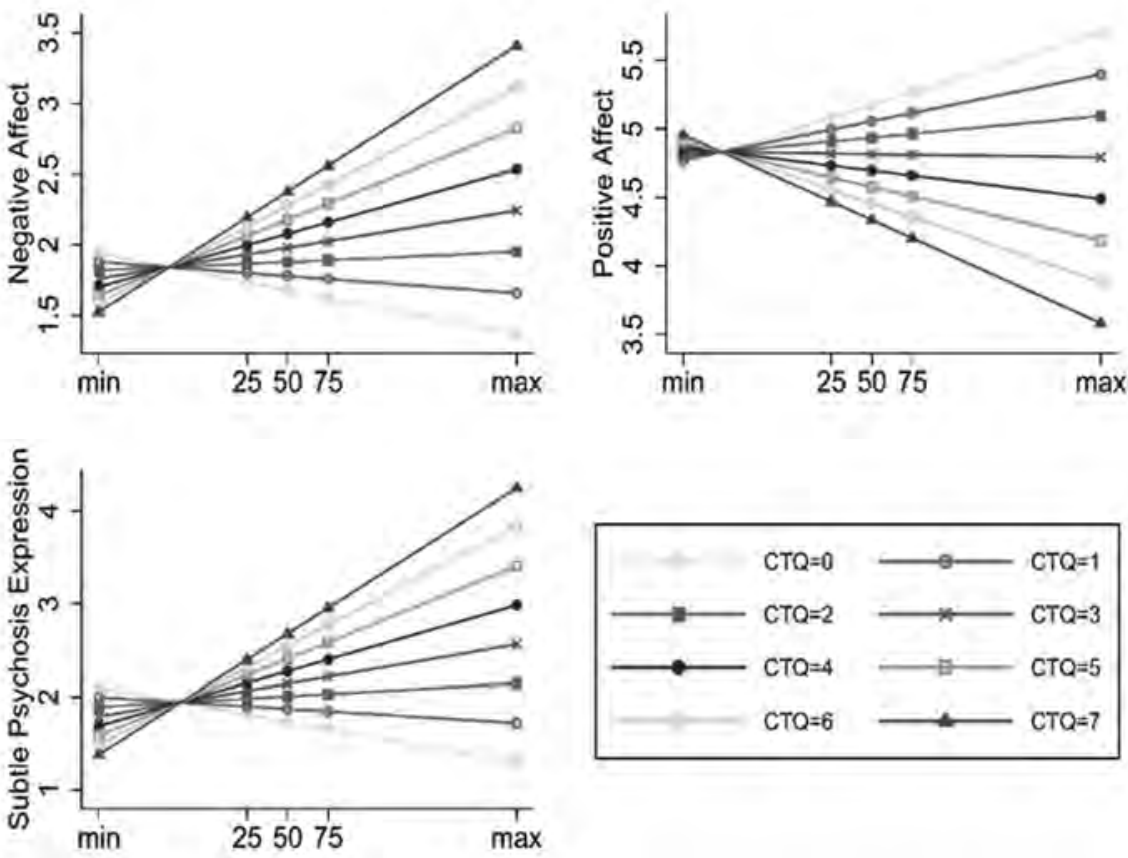

Marginal effect plots based on multilevel tobit regression of the interaction between continuous polygenic risk score for schizophrenia (x-axis) and continuous childhood adversity score on continuous measures of negative affect, positive affect and subtle psychosis expression, $y$-axis). For visualization purposes, margins at quartiles of PRS-S and standardized scores of CA from 0 to 7 were illustrated. CA: Childhood adversity, PRSS: Polygenic risk score for schizophrenia [i.e. range: $\min$ (minimum), 25 th percentile, 50 th percentile, 75 th percentile, and max (maximum)]. 
Table 3 Associations and interaction effects of CA and PRS-S with momentary mental state domains

\begin{tabular}{|c|c|c|c|c|c|c|c|c|c|}
\hline & \multicolumn{3}{|c|}{ Association with CA } & \multicolumn{3}{|c|}{ Association with PRS-S } & \multicolumn{3}{|c|}{$\begin{array}{c}\text { Interaction between PRS-S and } \\
\qquad \mathrm{CA}^{\dagger}\end{array}$} \\
\hline & $\mathrm{b}$ & $P$-value & $95 \% \mathrm{CI}$ & $\mathrm{b}$ & $P$-value & $95 \% \mathrm{CI}$ & $\mathrm{b}$ & $P$-value & $95 \% \mathrm{CI}$ \\
\hline Negative affect & 0.13 & $<0.001^{*}$ & $\begin{array}{c}0.07 \text { to } \\
0.19\end{array}$ & -0.02 & 0.502 & $\begin{array}{c}-0.08 \text { to } \\
0.04\end{array}$ & 0.07 & $0.013^{\circ}$ & $\begin{array}{c}0.01 \text { to } \\
0.13\end{array}$ \\
\hline Positive affect & -0.12 & $<0.001^{*}$ & $\begin{array}{c}-0.17 \text { to } \\
-0.06\end{array}$ & 0.08 & $0.003^{*}$ & $\begin{array}{c}0.03 \text { to } \\
0.14\end{array}$ & -0.05 & 0.043 & $\begin{array}{c}-0.10 \text { to }- \\
0.00\end{array}$ \\
\hline $\begin{array}{l}\text { Subtle psychosis } \\
\text { expression }\end{array}$ & 0.18 & $<0.001^{*}$ & $\begin{array}{l}0.10 \text { to } \\
0.26\end{array}$ & -0.03 & 0.547 & $\begin{array}{c}-0.11 \text { to } \\
0.06\end{array}$ & 0.11 & $0.007^{*}$ & $\begin{array}{c}0.03 \text { to } \\
0.19\end{array}$ \\
\hline
\end{tabular}

All analyses were adjusted for age and sex, ${ }^{\dagger}$ also adjusted for 2 principal components, "significant after controlling for family-wise type I error using the Bonferroni method (0.05/3=0.0167). CA: Childhood adversity, CI: Confidence interval, PRS-S: Polygenic risk score for schizophrenia

\section{Main associations and interactions of daily-life stressors and PRS-S on momentary mental state domains}

The overall mean and each of the daily-life stressors were associated with increased NA, decreased PA, and increased PE (Table S6). No gene-environment correlation was present as PRS-S was not associated with any of the daily-life stressors (overall stress: $\mathrm{b}=-0.02,95 \%$ $\mathrm{CI}=-0.06$ to $0.03, P=0.491$; event stress: $\mathrm{b}=0.01,95 \% \mathrm{CI}=-0.04$ to $0.06, P=0.672$; social stress: $\mathrm{b}=-0.03,95 \% \mathrm{CI}=-0.07$ to $0.02, P=0.267$; activity stress: $\mathrm{b}=-0.01,95 \% \mathrm{CI}$ $=-0.05$ to $0.03, P=0.571)$. No evidence for significant interaction effects between daily-life stressors and PRS-S was found (Table S6).

\section{Main associations and interactions of CA and PRS-S on stress-sensitivity measures}

CA was associated with increased stress-sensitivity measures, while PRS-S was only associated with increased PA stress-sensitivity. Evidence was found for significant gene-environment interaction. The associations between CA and stress-sensitivity measures were greater if individuals had higher PRS-S (Table 4).

\section{Discussion}

\section{Principal findings}

In this first study testing PRS-S for an interaction with early and late stressors (childhood adversity and minor daily-life stressors) in association with dynamic pluripotent mental processes in the largest EMA dataset to date, evidence emerged for an interaction between PRS-S and childhood adversity to influence momentary mental states (negative affect, positive affect, and subtle psychosis expression) and stress-sensitivity measures. 


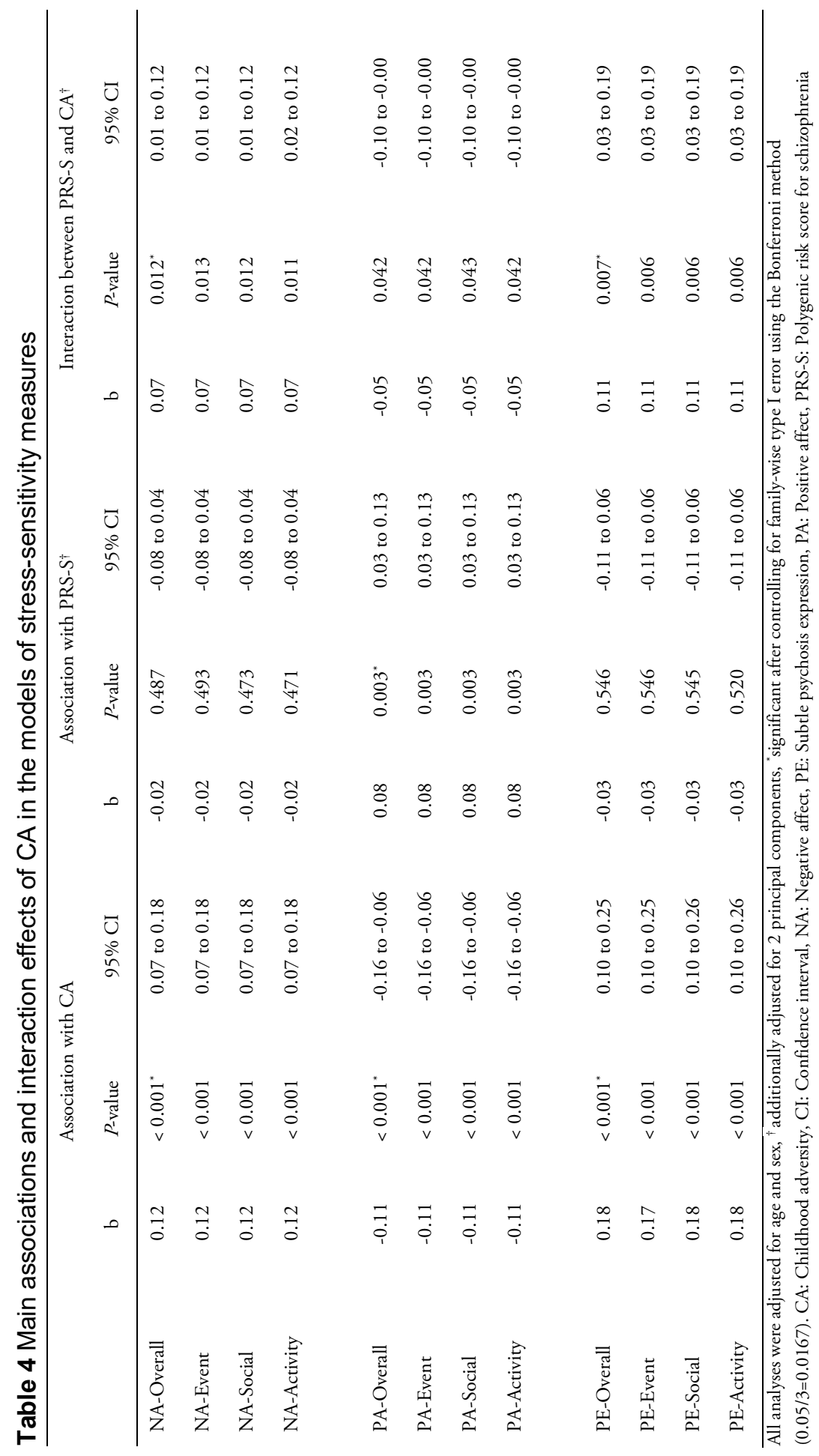




\section{Stress exposure and emotional processes}

In line with long-established findings from population-based datasets and samples of helpseeking adolescents and young adults ${ }^{9,15}$, we showed that minor daily-life stressors, regardless of the type of the stressor, were associated with all three domains of momentary mental states. More importantly, we provided further support for the shared vulnerability theory of mental disorders by demonstrating that CA was associated with NA, PA, and PE. These results echo recent findings from our line of research showing that $\mathrm{CA}$ is not exclusively associated with a specific mental disorder category, but rather with multidimensional psychopathology (cutting across diagnostic categories) in the general population, such as psychotic experiences, affective dysregulation, and negative symptoms $s^{52-57}$. Therefore, it is plausible to conceptualize that the sensitivity to daily-life stressors is molded by previous exposure to significant life stressors as discussed in the models of diathesis-stress ${ }^{14}$ and sensory processing sensitivity ${ }^{58}$. Furthermore, the exposome, a dense network of environmental exposures ${ }^{59,60}$, may contribute to a person's sensitivity to stress.

\section{Genetic vulnerability for schizophrenia moderates sensitivity to childhood adversity}

PRS-S, as anticipated, had no significant predictive power for the EMA outcomes with the exception of positive affect-related items that were positively associated with PRS-S. The positive association between PRS-S and positive affect might seem counterintuitive at first glance given that EMA studies have shown a decreased positive affect in patients with schizophrenia and PRS-S is associated with schizophrenia. However, emerging evidence suggests that the relation between PRS-S and symptom dimensions at the population level appears to be not following a simple logic. As this is the very first and the only EMA study investigating PRS-S, we could not make an exact comparison of our findings. Unfortunately, because most studies focus on psychopathology, it is also difficult to draw a parallel between our current results on positive affect and findings from studies investigating the association between PRS-S and symptom dimensions in healthy participants. These studies in healthy participants have shown inconsistent results ${ }^{61-66}$. Some reported no association between PRS$S$ and several symptoms dimensions ${ }^{63,67}$, while others reported negative associations between polygenic risk and schizotypy ${ }^{64,67}$. In addition, in the rare instances where it has been examined, PRS-S appear to contribute to abilities required for a creative profession ${ }^{68}$. Taken together, these findings in fact suggest a substantial fraction of pathoetiology may be explained by the influence of environment and the GxE. In agreement, our interaction analyses showed a consistent pattern. PRS-S moderated the influence of CA, but not the impact of minor daily-life stressors, for all momentary mental states, with negative affect and subtle psychosis expression reaching the Bonferroni-adjusted statistical significance level. 
This interaction effect was similarly present in sensitivity to overall-stress and consistently observed for sensitivity to event, activity, and social stress.

The current results showing a difference between the degree of genetic moderation of two stressors (CA and daily-life stressors) underscores the importance of the type, timing, and extent of stressor in mental health impact. This is consistent with the neurodevelopmental hypothesis ${ }^{69,70}$ that postulates that exposure to early-life stressors in neurodevelopmentally sensitive periods are more likely to disturb the balance of important stress systems and lead to enduring emotional and behavioral problems in later life. These findings, combined with a recent meta-analysis showing that patients with PSD experience more negative emotion and less positive emotion in daily life ${ }^{71}$, suggest that genetic and early adversities may have a permanent impact on mental wellbeing, resulting in a trait-like feature of person-specific alterations in emotional expression and psychosis proneness. An interesting question for future research spanning an extended period would be whether persistent low-threshold daily-life stressors may influence emotional reactivity toward mental ill health in the longterm or whether more serious life events are required to reach the threshold for clinical syndrome. Early candidate gene studies investigating the genetic moderation of mental health outcomes have generated mostly inconclusive findings because of methodological issues. COMT ${ }^{\text {Val158Met }} \mathrm{Val} / \mathrm{Val}$ carriers displayed increased paranoia in response to stress ${ }^{22}$, while, in another study, Met/Met genotype was associated with increased PA in response to experiencing positive events $\mathrm{s}^{72}$. Momentary stress interacted with genetic variation in the brain-derived neurotrophic factor gene, Met carriers reporting higher paranoia scores than Val carriers ${ }^{22}$. However, a recent study failed to replicate these findings, but showed an interaction between childhood trauma and RGS4, FKBP5, and $O X T R$, respectively ${ }^{73}$. As PRS-based approaches for testing $\mathrm{G} \times \mathrm{E}$ have recently emerged, no comparable EMA study was available. However, in line with our study, research showed that higher PRS (consisting of 13 genes previously associated with vulnerability to environmental exposure) increased the influence of CA on stress-sensitization ${ }^{74}$. Further, we recently showed evidence that the interaction between PRS-S and childhood adversity increases the likelihood of schizophrenia ${ }^{25}$.

Given the influence of psychosocial stressors on immune processes and hypothalamicpituitary axis modulation underlying the etiopathogenesis of $\mathrm{PSD}^{75}$, future studies embracing biologically-informative target approaches may exploit the unique ability of EMA to capture dynamic fluctuation of mental states, and combine the granular information with multiomics data (e.g., genome, proteome, and epigenome) to study candidate molecular 
mechanisms such as $F K B P 5^{76}$ and extend previous EMA work investigating cortisol reactivity to daily-life stressors in relation to $\mathrm{PSD}^{77}$.

\section{Pleiotropic influence of exposures and genetic vulnerability on psychopathology}

Our findings agree with the literature showing that the influence of $\mathrm{CA}^{78}$ and schizophrenia genetic liability $2,63,65$ on mental health in the general population is pleiotropic and converge on shared psychological constructs and multidimensional psychopathology in the causal path to PSD. Considering the fact that mental health phenotypes (EMA outcomes in our study) are associated with each other at both dimensional and diagnostic levels and thereby violating the assumption of independence of pleiotropy, it is also plausible to argue that these disorders defined at the symptom level might be different expressions (phenotypic presentations) of a substantially shared pathoetiology with varying outcomes due to disease modifiers rather than distinct entities ${ }^{79,80}$. Furthermore, in accordance with the idea of an affective pathway to psychosis, it is also plausible that the EMA outcomes may be temporally associated with each other, such that the interaction between PRS-S and childhood adversity may influence psychosis expression through negative affect.

A growing investment into transdiagnostic research of mental health will hopefully shed more light on this matter. Abundant evidence shows that the earliest psychopathological processes expressed before the prodrome of PSD are non-specific and include affective dysregulation, aberrant salience, and subtle cognitive disturbances ${ }^{79}$. In this regard, EMA outcomes capturing subtle and transitory mental states, such as emotional reactivity and stresssensitivity, are arguably more useful transdiagnostic phenotypes than static questionnairebased interval assessments to examine the contributions of environmental and genetic factors to variation in mental health at the community level ${ }^{26}$. As recently proposed ${ }^{81}$, multi-layered digital phenotyping via mobile devices may advance the RDoC work in the era of "Big Data" boosted by historic efforts of personalized medicine such as the National Institutes of Health initiative, the All of US research program.

\section{Limitations}

The current study provided the first insights into the influence of genetic regulation of exposure to stressors on dynamic mental states by taking advantage of a unique population dataset with fine-grained phenotyping. However, several methodological considerations should be noted. First, although one of the strengths is that the sample comprises individuals at an age range when mental disorders often emerge, it is also possible that the association between PRS-S and stress may change as a function of aging and cumulative stressor load. 
Second, the retrospective collection of CA might be subject to recall and response biases; however, it is not intuitive how these would be differential with regard to EMA outcomes or PRS-S, or their interaction. Third, daily-life stressors might not only influence momentary mental states but might also be influenced by them. Fourth, EMA provides a unique opportunity to focus on moment-to-moment fluctuations of mental states; nevertheless, it may be more difficult to detect psychosis proneness than emotional reactivity in the general population.

To conclude, this observational study suggests that the exposure to childhood adversities, especially in individuals with high molecular genetic risk for schizophrenia, is associated with emotion dysregulation and psychosis proneness. Further preregistered confirmatory research is required to validate these findings.

\section{Acknowledgments}

The authors thank Jill Ielegems, Katrien Lyssens, Davinia Verhoeven, and Debora op't Eijnde for data-collection. The authors would like to acknowledge that the East Flanders Prospective Twin Survey (EFPTS) is partly supported by the Association for Scientific Research in Multiple Births and that the TwinssCan project is part of the European Community's Seventh Framework Program under grant agreement No. HEALTH-F22009-241909 (Project EU-GEI). 


\section{References}

1. van Os J, Kenis G, Rutten BP. The environment and schizophrenia. Nature. 2010;468(7321):203212.

2. van Os J, van der Steen Y, Islam MA, et al. Evidence that polygenic risk for psychotic disorder is expressed in the domain of neurodevelopment, emotion regulation and attribution of salience. Psychol Med. 2017;47(14):2421-2437.

3. Nivard MG, Gage SH, Hottenga JJ, et al. Genetic overlap between schizophrenia and developmental psychopathology: longitudinal and multivariate polygenic risk prediction of common psychiatric traits during development. Schizophr Bull. 2017;43(6):1197-1207.

4. Misiak B, Krefft M, Bielawski T, Moustafa AA, Sasiadek MM, Frydecka D. Toward a unified theory of childhood trauma and psychosis: A comprehensive review of epidemiological, clinical, neuropsychological and biological findings. Neurosci Biobehav Rev. 2017;75:393-406.

5. Brainstorm C, Anttila V, Bulik-Sullivan B, et al. Analysis of shared heritability in common disorders of the brain. Science. 2018;360(6395):eaap8757.

6. Nelson B, McGorry PD, Wichers M, Wigman JTW, Hartmann JA. Moving From Static to Dynamic Models of the Onset of Mental Disorder: A Review. JAMA psychiatry. 2017;74(5):528-534.

7. Myin-Germeys I, van Os J. Stress-reactivity in psychosis: evidence for an affective pathway to psychosis. Clinical psychology review. 2007;27(4):409-424

8. Collip D, Nicolson N, Lardinois M, Lataster T, Van Os J, Myin-Germeys I. Daily cortisol, stress reactivity and psychotic experiences in individuals at above average genetic risk for psychosis. Psychol Med. 2011;41(11):2305-2315.

9. Lataster $\mathrm{T}$, Wichers $\mathrm{M}$, Jacobs $\mathrm{N}$, et al. Does reactivity to stress cosegregate with subclinical psychosis? A general population twin study. Acta Psychiatr Scand. 2009;119(1):45-53.

10. Myin-Germeys I, van Os J, Schwartz JE, Stone AA, Delespaul PA. Emotional reactivity to daily life stress in psychosis. Arch Gen Psychiatry. 2001;58(12):1137-1144

11. Myin-Germeys I, Peeters F, Havermans R, et al. Emotional reactivity to daily life stress in psychosis and affective disorder: an experience sampling study. Acta Psychiatr Scand. 2003;107(2):124-131.

12. Myin-Germeys I, Delespaul P, van Os J. Behavioural sensitization to daily life stress in psychosis. Psychol Med. 2005;35(5):733-741.
13. Palmier-Claus JE, Dunn G, Lewis SW. Emotiona and symptomatic reactivity to stress in individuals at ultra-high risk of developing psychosis. Psychol Med. 2012;42(5):1003-1012.

14. Pruessner M, Cullen AE, Aas M, Walker EF. The neural diathesis-stress model of schizophrenia revisited: An update on recent findings considering illness stage and neurobiological and methodological complexities. Neurosci Biobehav Rev. 2017;73:191-218

15. Rauschenberg C, van Os J, Cremers D, Goedhart M, Schieveld JNM, Reininghaus U. Stress sensitivity as a putative mechanism linking childhood trauma and psychopathology in youth's daily life. Acta Psychiatr Scand. 2017;136(4):373388.

16. Reininghaus U, Gayer-Anderson C, Valmaggia L, et al. Psychological processes underlying the association between childhood trauma and psychosis in daily life: an experience sampling study. Psychol Med. 2016;46(13):2799-2813.

17. Glaser JP, van Os J, Portegijs PJ, Myin-Germeys I. Childhood trauma and emotional reactivity to daily life stress in adult frequent attenders of general practitioners. J Psychosom Res. 2006;61(2):229236.

18. Wichers M, Schrijvers D, Geschwind N, et al. Mechanisms of gene-environment interactions in depression: evidence that genes potentiate multiple sources of adversity. Psychol Med. 2009;39(7):1077-1086

19. Lardinois M, Lataster T, Mengelers R, Van Os J, Myin-Germeys I. Childhood trauma and increased stress sensitivity in psychosis. Acta Psychiatr Scand. 2011;123(1):28-35.

20. Wigman JT, Collip D, Wichers M, et al. Altered transfer of momentary mental states (ATOMS) as the basic unit of psychosis liability in interaction with environment and emotions. PloS one. 2013;8(2):e54653.

21. van Winkel R, Henquet C, Rosa A, et al. Evidence that the COMT(Val158Met) polymorphism moderates sensitivity to stress in psychosis: an experience-sampling study. $A m J M e d$ Genet $B$ Neuropsychiatr Genet. 2008;147B(1):10-17.

22. Simons CJ, Wichers M, Derom C, et al. Subtle gene-environment interactions driving paranoia in daily life. Genes Brain Behav. 2009;8(1):5-12.

23. Henquet C, Rosa A, Delespaul P, et al. COMT Val158Met moderation of cannabis-induced psychosis: a momentary assessment study of 
'switching on'hallucinations in the flow of daily life. Acta Psychiat Scand. 2009;119(2):156-160.

24. Iyegbe C, Campbell D, Butler A, Ajnakina O, Sham $P$. The emerging molecular architecture of schizophrenia, polygenic risk scores and the clinical implications for GxE research. Soc Psychiatry Psychiatr Epidemiol. 2014;49(2):169-182.

25. Guloksuz S, Pries LK, Delespaul P, et al. Examining the independent and joint effects of molecular genetic liability and environmental exposures in schizophrenia: results from the EUGEI study. World Psychiatry. 2019;18(2):173-182.

26. Fox E, Beevers CG. Differential sensitivity to the environment: contribution of cognitive biases and genes to psychological wellbeing. Mol Psychiatry. 2016;21(12):1657-1662.

27. Insel TR. The NIMH Research Domain Criteria (RDoC) Project: precision medicine for psychiatry. The American journal of psychiatry. 2014;171(4):395-397.

28. Pries LK, Guloksuz S, Menne-Lothmann C, et al. White noise speech illusion and psychosis expression: An experimental investigation of psychosis liability. PloS one. 2017;12(8):e0183695.

29. Derom C, Thiery E, Peeters H, Vlietinck R, Defoort P, Frijns JP. The East Flanders Prospective Twin Survey (EFPTS): an actual perception. Twin Res Hum Genet. 2013;16(1):58-63.

30. Myin-Germeys I, Oorschot M, Collip D, Lataster J, Delespaul P, van Os J. Experience sampling research in psychopathology: opening the black box of daily life. Psychol Med. 2009;39(9):1533-1547.

31. Shiffman S, Stone AA, Hufford MR. Ecological momentary assessment. Annu Rev Clin Psychol. 2008;4:1-32.

32. Palmier-Claus JE, Myin-Germeys I, Barkus E, et al. Experience sampling research in individuals with mental illness: reflections and guidance. Acta Psychiatr Scand. 2011;123(1):12-20.

33. Verhagen SJ, Hasmi L, Drukker M, van Os J, Delespaul PA. Use of the experience sampling method in the context of clinical trials. Evid Based Ment Health. 2016;19(3):86-89.

34. Verhagen SJ, Berben JA, Leue C, et al. Demonstrating the reliability of transdiagnostic mHealth Routine Outcome Monitoring in mental health services using experience sampling technology. PloS one. 2017;12(10):e0186294.

35. Henquet C, van Os J, Kuepper R, et al. Psychosis reactivity to cannabis use in daily life: an experience sampling study. Br J Psychiatry. 2010;196(6):447453.

36. Vaessen T, van Nierop M, Decoster J, et al. Is sensitivity to daily stress predictive of onset or persistence of psychopathology? Eur Psychiatry. 2017;45:167-173

37. Bernstein DP, Stein JA, Newcomb MD, et al. Development and validation of a brief screening version of the Childhood Trauma Questionnaire. Child Abuse Negl. 2003;27(2):169-190

38. Lecei A, Decoster J, De Hert M, et al. Evidence that the association of childhood trauma with psychosis and related psychopathology is not explained by gene-environment correlation: A monozygotic twin differences approach. Schizophr Res. 2019;205:58 62.

39. Purcell S, Neale B, Todd-Brown K, et al. PLINK: a tool set for whole-genome association and population-based linkage analyses. Am J Hum Genet. 2007;81(3):559-575.

40. Price AL, Patterson NJ, Plenge RM, Weinblatt ME, Shadick NA, Reich D. Principal components analysis corrects for stratification in genome-wide association studies. Nat Genet. 2006;38(8):904909.

41. Das S, Forer L, Schonherr S, et al. Next-generation genotype imputation service and methods. Nat Genet. 2016;48(10):1284-1287.

42. Pardinas AF, Holmans P, Pocklington AJ, et al. Common schizophrenia alleles are enriched in mutation-intolerant genes and in regions under strong background selection. Nat Genet. 2018;50(3):381-389.

43. Price AL, Weale ME, Patterson N, et al. Longrange $L D$ can confound genome scans in admixed populations. Am J Hum Genet. 2008;83(1):132135; author reply 135-139.

44. Wray NR, Lee SH, Mehta D, Vinkhuyzen AA, Dudbridge F, Middeldorp CM. Research review: Polygenic methods and their application to psychiatric traits. J Child Psychol Psychiatry. 2014;55(10):1068-1087.

45. Simons CJ, Wichers M, Derom C, et al. Subtle gene-environment interactions driving paranoia in daily life. Genes, Brain Behavior. 2009;8(1):5-12.

46. Snijders TAB, Bosker RJ. Multilevel analysis: an introduction to basic and advanced multilevel modeling. London: SAGE Publications Ltd; 1999.

47. Carlin JB, Gurrin LC, Sterne JA, Morley R, Dwyer T. Regression models for twin studies: a critical review. Int J Epidemiol. 2005;34(5):1089-1099.

48. Tobin J. Estimation of Relationships for Limited Dependent Variables. Econometrica. 1958;26(1):24-36

49. Stata Statistical Software [computer program]. College Station, TX: StataCorp LLC; 2017.

50. Reininghaus U, Kempton MJ, Valmaggia L, et al. Stress Sensitivity, Aberrant Salience, and Threat 
Anticipation in Early Psychosis: An Experience Sampling Study. Schizophr Bull. 2016;42(3):712722.

51. Keller MC. Genex environment interaction studies have not properly controlled for potential confounders: the problem and the (simple) solution. Biol Psychiatry. 2014;75(1):18-24.

52. Guloksuz $S$, van Nierop M, Lieb R, van Winkel R, Wittchen $\mathrm{H}-\mathrm{U}$, van Os J. Evidence that the presence of psychosis in non-psychotic disorder is environment-dependent and mediated by severity of non-psychotic psychopathology. Psychological medicine. 2015;45(11):2389-2401.

53. Pries LK, Guloksuz S, Ten Have M, et al. Evidence That Environmental and Familial Risks for Psychosis Additively Impact a Multidimensional Subthreshold Psychosis Syndrome. Schizophr Bull. 2018;44(4):710-719.

54. Guloksuz S, van Nierop M, Bak M, et al. Exposure to environmental factors increases connectivity between symptom domains in the psychopathology network. BMC psychiatry. 2016;16(1):223.

55. Radhakrishnan R, Guloksuz S, Ten Have M, et al. Interaction between environmental and familial affective risk impacts psychosis admixture in states of affective dysregulation. Psychol Med. 2019;49(11):1879-1889.

56. van Nierop M, Bak M, de Graaf R, et al. The functional and clinical relevance of childhood trauma-related admixture of affective, anxious and psychosis symptoms. Acta Psychiatr Scand. 2016;133(2):91-101.

57. van Nierop M, Viechtbauer W, Gunther N, et al. Childhood trauma is associated with a specific admixture of affective, anxiety, and psychosis symptoms cutting across traditional diagnostic boundaries. Psychological medicine. 2015;45(6):1277-1288.

58. Aron EN, Aron A, Jagiellowicz J. Sensory processing sensitivity: a review in the light of the evolution of biological responsivity. Pers Soc Psychol Rev. 2012;16(3):262-282.

59. Pries L-K, Lage-Castellanos A, Delespaul P, et al. Estimating Exposome Score for Schizophrenia Using Predictive Modeling Approach in Two Independent Samples: The Results From the EUGEI Study. Schizophr Bulletin. 2019;45(5):960965.

60. Guloksuz S, Rutten BPF, Pries LK, et al. The Complexities of Evaluating the Exposome in Psychiatry: A Data-Driven Illustration of Challenges and Some Propositions for Amendments. Schizophr Bull. 2018;44(6):11751179.
61. Mistry S, Harrison JR, Smith DJ, Escott-Price V, Zammit $S$. The use of polygenic risk scores to identify phenotypes associated with genetic risk of schizophrenia: systematic review. Schizophrenia research. 2018;197:2-8.

62. Hatzimanolis A, Bhatnagar P, Moes A, et al. Common genetic variation and schizophrenia polygenic risk influence neurocognitive performance in young adulthood. Am J Med Genet B Neuropsychiatr Genet. 2015;168B(5):392-401.

63. Jones HJ, Stergiakouli E, Tansey KE, et al. Phenotypic Manifestation of Genetic Risk for Schizophrenia During Adolescence in the General Population. JAMA psychiatry. 2016;73(3):221-228.

64. Hatzimanolis A, Avramopoulos D, Arking DE, et al. Stress-dependent association between polygenic risk for schizophrenia and schizotypal traits in young army recruits. Schizophrenia Bull. 2017;44(2):338-347.

65. Riglin L, Collishaw S, Richards A, et al. Schizophrenia risk alleles and neurodevelopmental outcomes in childhood: a population-based cohort study. Lancet Psychiat. 2017;4(1):57-62.

66. Nivard MG, Gage SH, Hottenga JJ, et al. Genetic overlap between schizophrenia and developmental psychopathology: longitudinal and multivariate polygenic risk prediction of common psychiatric traits during development. Schizophr Bull. 2017;43(6):1197-1207.

67. van Os J, Pries L-K, Delespaul P, et al. Replicated evidence that endophenotypic expression of schizophrenia polygenic risk is greater in healthy siblings of patients compared to controls, suggesting gene-environment interaction. The EUGEI study. Psychological medicine. 2019:1-14.

68. Power RA, Steinberg S, Bjornsdottir G, et al. Polygenic risk scores for schizophrenia and bipolar disorder predict creativity. Nat Neurosci. 2015;18(7):953-955.

69. Murray RM, Lewis SW. Is schizophrenia a neurodevelopmental disorder? Br Med J (Clin Res Ed). 1987;295(6600):681.

70. Weinberger DR. The neurodevelopmental origins of schizophrenia in the penumbra of genomic medicine. World Psychiatry. 2017;16(3):225-226.

71. Cho H, Gonzalez R, Lavaysse LM, Pence S, Fulford D, Gard DE. Do people with schizophrenia experience more negative emotion and less positive emotion in their daily lives? A meta-analysis of experience sampling studies. Schizophr Res. 2017;183:49-55.

72. Wichers M, Aguilera M, Kenis G, et al. The catechol-O-methyl transferase Val158Met polymorphism and experience of reward in the flow 
of daily life. Neuropsychopharmacology. 2008;33(13):3030-3036.

73. Cristobal-Narvaez P, Sheinbaum T, Myin-Germeys $\mathrm{I}$, et al. The role of stress-regulation genes in moderating the association of stress and daily-life psychotic experiences. Acta Psychiatr Scand. 2017;136(4):389-399.

74. Keers R, Pluess M. Childhood quality influences genetic sensitivity to environmental influences across adulthood: a life-course genex environment interaction study. Dev Psychopathol. 2017;29(5):1921-1933.

75. Radhakrishnan R, Kaser M, Guloksuz S. The link between the immune system, environment, and psychosis. Schizophr Bull. 2017;43(4):693-697.

76. Zannas A, Binder E. Gene-environment interactions at the FKBP5 locus: Sensitive periods, mechanisms and pleiotropism. Genes, Brain and Behavior. 2014;13(1):25-37.

77. Vaessen T, Kasanova Z, Hernaus D, et al. Overall cortisol, diurnal slope, and stress reactivity in psychosis: an experience sampling approach. Psychoneuroendocrinology. 2018;96:61-68.

78. Isvoranu AM, Borsboom D, van Os J, Guloksuz S. A Network Approach to Environmental Impact in Psychotic Disorder: Brief Theoretical Framework. Schizophr Bull. 2016;42(4):870-873.

79. Guloksuz S, van Os J. The slow death of the concept of schizophrenia and the painful birth of the psychosis spectrum. Psychol Med. 2018;48(2):229-244.

80. Guloksuz S, Pries LK, van Os J. Application of network methods for understanding mental disorders: pitfalls and promise. Psychol Med. 2017;47(16):2743-2752.

81. Torous J, Onnela JP, Keshavan M. New dimensions and new tools to realize the potential of RDoC: digital phenotyping via smartphones and connected devices. Transl Psychiatry. 2017;7(3):e1053 


\section{Supplementary material}

Genotyping, quality control, imputation, and PRS

Part 1 Target Genotype Data Processing

Quality control for genotype data before imputation

TwinssCan data were genotyped on two chips: Infinium CoreExome-24 Kit (570,038 genotyped variants for 634 participants) and Infinium PsychArray-24 Kit (588,628 genotyped variants for 82 participants). These two datasets from different chips were quality controlled separately using PLINK v1.9 ${ }^{1}$. Several pre-imputation quality control (QC) steps were applied to the datasets. In detail, single nucleotide polymorphisms (SNPs) and samples with call rates below $95 \%$ and $98 \%$, respectively, were removed. A strict SNP QC only for subsequent sample QC steps was conducted. This involved a minor allele frequency (MAF) threshold $>10 \%$ and a Hardy-Weinberg equilibrium (HWE) $P$-value $>10^{-5}$, followed by linkage disequilibrium (LD) based $\mathrm{SNP}$ pruning $\left(\mathrm{R}^{2}<0.5\right)$. This resulted in $-58 \mathrm{~K}$ SNPs to assess sex errors $(\mathrm{n}=8)$, heterozygosity $[\mathrm{F}<5 \mathrm{x}$ the standard deviation $(\mathrm{SD}), \mathrm{n}=3]$, homozygosity $(\mathrm{F}>5 \mathrm{x} \mathrm{SD})$, and relatedness by pairwise identity by descent (IBD) values (monozygotic: $\hat{p}<0.9$ dizygotic and full siblings: $\hat{p}>0.650 \mathrm{p}<$ $0.35, \mathrm{n}=5$ ). After removing failing samples, a regular SNP QC was performed (SNP call rate $>98 \%, \mathrm{HWE} p>1 \mathrm{e}-06, \mathrm{MAF}>1 \%$ ). Next, strand ambiguous SNPs and duplicate SNPs were removed. 270,976 variants and 610 individuals from chip 1, and 273,523 variants and 78 individuals from chip 2 passed these QC steps.

\section{Imputation on Michigan server}

The two QC-ed datasets were converted into *.VCF files chunked by chromosome and imputed using the following settings: reference panel as HRC R1.1 2016; phasing as Eagle v2.3; population as European; model as QC \& imputation. The imputation step resulted in 39,117,084 single nucleotide polymorphisms (SNPs). The general imputation quality is shown in Supplementary Figure 1 .

Supplementary Figure 1 Correlation of SNPsMAF from chip1 (Supplementary Figure $1 \mathrm{~A}$ ) and chip 2 (Supplementary Figure $1 \mathrm{~B}$ ) dataset with the reference MAF



Supplementary Figure 1 B Correlation of SNPs MAF in chip2 dataset with the reference MAF

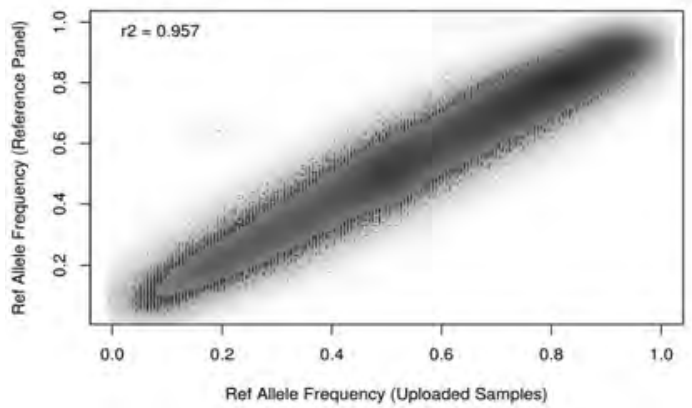

Quality control after imputation

The VCF files were firstly converted into PLINK best guess (hard call) genotypes by PLINK --vcf function. In the meantime, poor quality SNPs were excluded: multiallelic SNPs, SNPs with a minor allele frequency $(\mathrm{MAF})<0.01$ or $\mathrm{INFO}<0.8$, and strand ambiguous AT/CG SNPs. The files were merged, remaining SNPs that passed QC from both arrays and another exclusion on MAF $(<0.01)$ and Hardy Weinberger equilibrium (HWE) $(<10 \mathrm{e}-6)$ in the whole dataset was performed. Finally, there were 3,407,392 SNPs and 695 individuals

Principal components analyses (PCA) The principal components (PCs) analyses within TwinssScan samples, and TwinssScan samples along with Hapmap3 (as can be found online $e^{2}$ populations were conduct by EIGENSTRAT ${ }^{3}$. A strict selection for SNPs which overlap with Hapmap3 SNPs list were conduct: 1 . MAF $>0.05$, HWE $>0.001 ; 2$. Removal of 20 long LD regions (Supplementary Table 1); 3. $\mathrm{LD}$ pruned with an $\mathrm{R}^{2}$ of 0.5 ; which resulting 95,466 best quality genotyped SNPs used to calculate genetic PCs. PCs were firstly calculated with hapmap3 population to exclude European ethnic outliers: exceeding 10 times the standard 
deviation of Utah residents with Northern and Western European ancestry from the CEPH collection (CEU) and Toscani in Italia (TSI) populations for first 4 PCs. The first 2 PCs explained $>80 \%$ of the total variance (see Supplementary Table 2; model 1). Five individuals are excluded from this dataset. Secondly, another PCA was conducted using the same SNP list $(\mathrm{n}=95,466)$ but calculated only in TwinssCan. The first 10 PCs explained $>50 \%$ of the total variance (see Supplementary Table 2; model 2). Seven individuals were further considered as ethnic outliers by the first 4 PCs exceeding 3 times the standard deviation in the TwinssCan sample, and were excluded from this study. In the end, 688 individuals remained (see Supplementary Figure 2). After post-imputation quality control step, PCA was conducted using the same SNP list $(\mathrm{n}=95,466)$ within TwinssCan samples $(\mathrm{n}=688)$, and first two genetic PCs were further used to correct population stratification.

Supplementary Table 120 complex-LD regions and long-range LD regions which were exlcued from PRS analysis.

\begin{tabular}{c|c}
\hline Chromosome & Base pair position (start poiny to end point) \\
\hline 1 & $48000000-52000000$ \\
2 & $86000000-100500000$ \\
3 & $183000000-190000000$ \\
3 & $47500000-50000000$ \\
5 & $83500000-87000000$ \\
5 & $44500000-50500000$ \\
6 & $129000000-132000000$ \\
6 & $25500000-33500000$ \\
6 & $57000000-64000000$ \\
7 & $140000000-142500000$ \\
8 & $55000000-66000000$ \\
8 & $8000000-12000000$ \\
8 & $43000000-50000000$ \\
10 & $112000000-115000000$ \\
11 & $37000000-43000000$ \\
12 & $87500000-90500000$ \\
20 & $33000000-40000000$ \\
8 & $32000000-34500000$ \\
17 & $8135000-12000000$ \\
& $40900000-45000000$ \\
\hline
\end{tabular}

Supplementary Figure 2 The first and second principal component of TwinssCan data (with identified ethnic outliers) along with hapmap3 populations.

Supplementary Table 2 Eigenvalues and proportion variance explained for the first 20 PCs from PCA analyses.

\begin{tabular}{|c|c|c|c|c|c|c|}
\hline \multirow[t]{2}{*}{ PCs } & \multicolumn{3}{|c|}{$\begin{array}{l}\text { model 1: PCA with TwinssCan and hapmap3 } \\
\text { population }\end{array}$} & \multicolumn{3}{|c|}{ model 2: PCA with TwinssCan cohort } \\
\hline & Eigenvalues & $\begin{array}{l}\text { proportion } \\
\text { variance }\end{array}$ & $\begin{array}{l}\text { accumulated } \\
\text { variance }\end{array}$ & Eigenvalues & $\begin{array}{l}\text { proportion } \\
\text { variance }\end{array}$ & $\begin{array}{l}\text { accumulated } \\
\text { variance }\end{array}$ \\
\hline PC1 & 164.209 & 0.504 & 0.505 & 3.040 & 0.065 & 0.065 \\
\hline PC2 & 101.007 & 0.310 & 0.815 & 2.748 & 0.058 & 0.123 \\
\hline PC3 & 8.521 & 0.026 & 0.841 & 2.554 & 0.054 & 0.178 \\
\hline PC4 & 7.795 & 0.023 & 0.865 & 2.482 & 0.053 & 0.231 \\
\hline PC5 & 7.152 & 0.021 & 0.887 & 2.451 & 0.052 & 0.284 \\
\hline PC6 & 2.773 & 0.008 & 0.896 & 2.427 & 0.051 & 0.336 \\
\hline PC7 & 2.740 & 0.008 & 0.904 & 2.420 & 0.051 & 0.388 \\
\hline PC8 & 2.692 & 0.008 & 0.912 & 2.364 & 0.050 & 0.438 \\
\hline PC9 & 2.588 & 0.007 & 0.920 & 2.334 & 0.049 & 0.488 \\
\hline PC10 & 2.490 & 0.007 & 0.928 & 2.210 & 0.047 & 0.536 \\
\hline PC11 & 2.471 & 0.007 & 0.936 & 2.202 & 0.047 & 0.583 \\
\hline $\mathrm{PC} 12$ & 2.430 & 0.007 & 0.943 & 2.188 & 0.046 & 0.630 \\
\hline PC13 & 2.390 & 0.007 & 0.950 & 2.180 & 0.046 & 0.676 \\
\hline PC14 & 2.351 & 0.007 & 0.958 & 2.174 & 0.046 & 0.723 \\
\hline PC15 & 2.345 & 0.007 & 0.965 & 2.167 & 0.046 & 0.769 \\
\hline PC16 & 2.312 & 0.007 & 0.972 & 2.158 & 0.046 & 0.815 \\
\hline PC17 & 2.264 & 0.006 & 0.979 & 2.152 & 0.046 & 0.862 \\
\hline PC18 & 2.259 & 0.006 & 0.986 & 2.149 & 0.046 & 0.908 \\
\hline PC19 & 2.243 & 0.006 & 0.993 & 2.147 & 0.045 & 0.954 \\
\hline PC20 & 2.224 & 0.006 & 1 & 2.145 & 0.045 & 1 \\
\hline
\end{tabular}

Part 2 Training schizophrenia GWAS summary statistic processing

Training data quality control

PRS-S were calculated based on the summary statistics from the Psychiatric Genetics Consortium-2 SZ and the CLOZUK sample (schizophrenia cases from the UK) ${ }^{4}$. GWAS summary statistic underwent multiple quality control steps and the odds ratios (ORs) were converted into beta values. The quality control criteria were following: 1) insertions and deletions, ambiguous SNPs; 2) SNPs with a MAF $<0.01$ and SNP with an imputation quality $\left(\mathrm{R}^{2}\right)<0.9$ in training and target datasets; 3) Overlapping SNPs between the schizophrenia GWAS (training dataset), 1000 genomes (reference dataset), and our dataset (target dataset) were selected; 4) SNPs located in complex-LD regions $s^{5}$ were excluded (Supplementary Table 1). These SNPs were clumped in two rounds using PLINK's clump function; round 1 with the default parameters (physical distance threshold $250 \mathrm{~kb}$ and $\mathrm{LD}$ threshold $\left(\mathrm{R}^{2}\right)<0.5(--\mathrm{clump}-\mathrm{kb}$ 250 --clump-r2 0.5); round 2 with a physical distance threshold of 5,000kb and LD threshold $\left(\mathrm{R}^{2}\right)<0.2$ (--clump-kb 5000 --clumpr2 0.2); resulting in 88,736 SNPs for PRS-S calculation.

\section{Calculating PRS}

The beta-values, effective allele, and P-value were extracted from all summary statistics. The PRS was calculated using PLINK's score function for schizophrenia P-value threshold $=0.05$. 
Supplementary Figure 3 Scatter plots of momentary mental state domains and PRS-S with lines of best fit at $\mathrm{CA}$ quartiles in raw data.
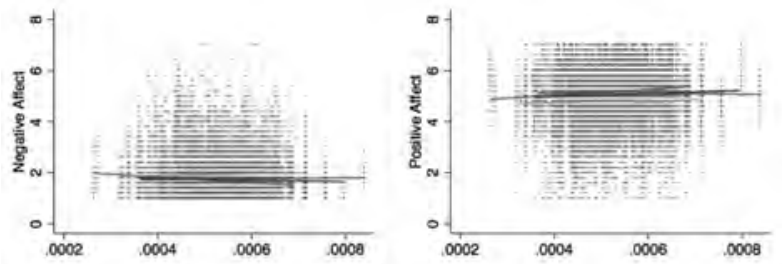

Interaction between polygenic risk for schizophrenia (x-axis) and childhood adversity on negative affect, positive affect and subtle psychosis expression (y-axis), CA: Childhood adversity (i.e. range: smaller than 25 th percentile, between 25 th percentile and 50th percentile, between 50 th percentile and 75 th percentile, and higher than

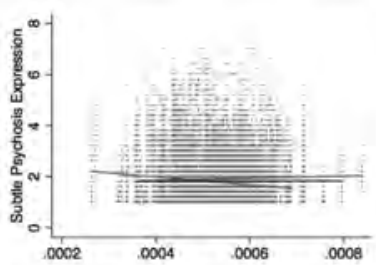
75th percentile), PRS-S: Polygenic risk score for schizophrenia.

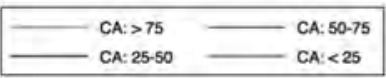

Supplementary Table 3 Childhood adversity subscales

\begin{tabular}{lccccc}
\hline & $\begin{array}{c}\text { Emotional abuse } \\
(\mathrm{n}=593)\end{array}$ & $\begin{array}{c}\text { Physical abuse } \\
(\mathrm{N}=593)\end{array}$ & $\begin{array}{c}\text { Sexual Abuse } \\
(\mathrm{N}=593)\end{array}$ & $\begin{array}{c}\text { Emotional neglect } \\
(\mathrm{n}=593)\end{array}$ & $\begin{array}{c}\text { Physical neglect } \\
(\mathrm{N}=593)\end{array}$ \\
\hline None & $405(68 \%)$ & $569(96 \%)$ & $559(94 \%)$ & $347(59 \%)$ & $501(84 \%)$ \\
Present & $188(32 \%)$ & $24(4 \%)$ & $34(6 \%)$ & $246(41 \%)$ & $92(16 \%)$ \\
Low & $132(70 \%)$ & $17(71 \%)$ & $21(62 \%)$ & $202(82 \%)$ & $64(70 \%)$ \\
Moderate & $35(19 \%)$ & $5(21 \%)$ & $9(26 \%)$ & $32(13 \%)$ & $20(22 \%)$ \\
Severe & $21(11 \%)$ & $2(8 \%)$ & $4(12 \%)$ & $12(5 \%)$ & $8(9 \%)$ \\
\hline
\end{tabular}

Emotional abuse: None = 5-8, Low $=$ 9-12, Moderate $=13-15$, Severe $\geq 16 ;$ Physical abuse: None = 5-7, Low $=8-9$, Moderate $=$ 10-12, Severe $\geq 13$; Sexual abuse: None $=5$, Low $=6-7$, Moderate $=8-12$, Severe $\geq 13$; Emotional neglect: None $=5-9$, Low $=10$ 14 , Moderate $=15-17$, Severe $\geq 18$; Physical neglect: None $=5-7$, Low $=8-9$, Moderate $=10-12$, Severe $\geq 13$

Supplementary Table 4 Correlation matrix between momentary mental state domains

\begin{tabular}{lccc}
\hline & $\begin{array}{c}\text { Negative affect } \\
\text { rho, p-value }\end{array}$ & $\begin{array}{c}\text { Positive affect } \\
\text { rho, p-value }\end{array}$ & $\begin{array}{c}\text { Subtle psychosis expression } \\
\text { rho, p-value }\end{array}$ \\
\hline Negative affect & 1 & - & -- \\
Positive affect & $-0.43,<0.001$ & 1 & -- \\
Subtle psychosis expression & $0.52,<0.001$ & $-0.22,<0.001$ & 1 \\
\hline
\end{tabular}

rho: Spearman's correlation 


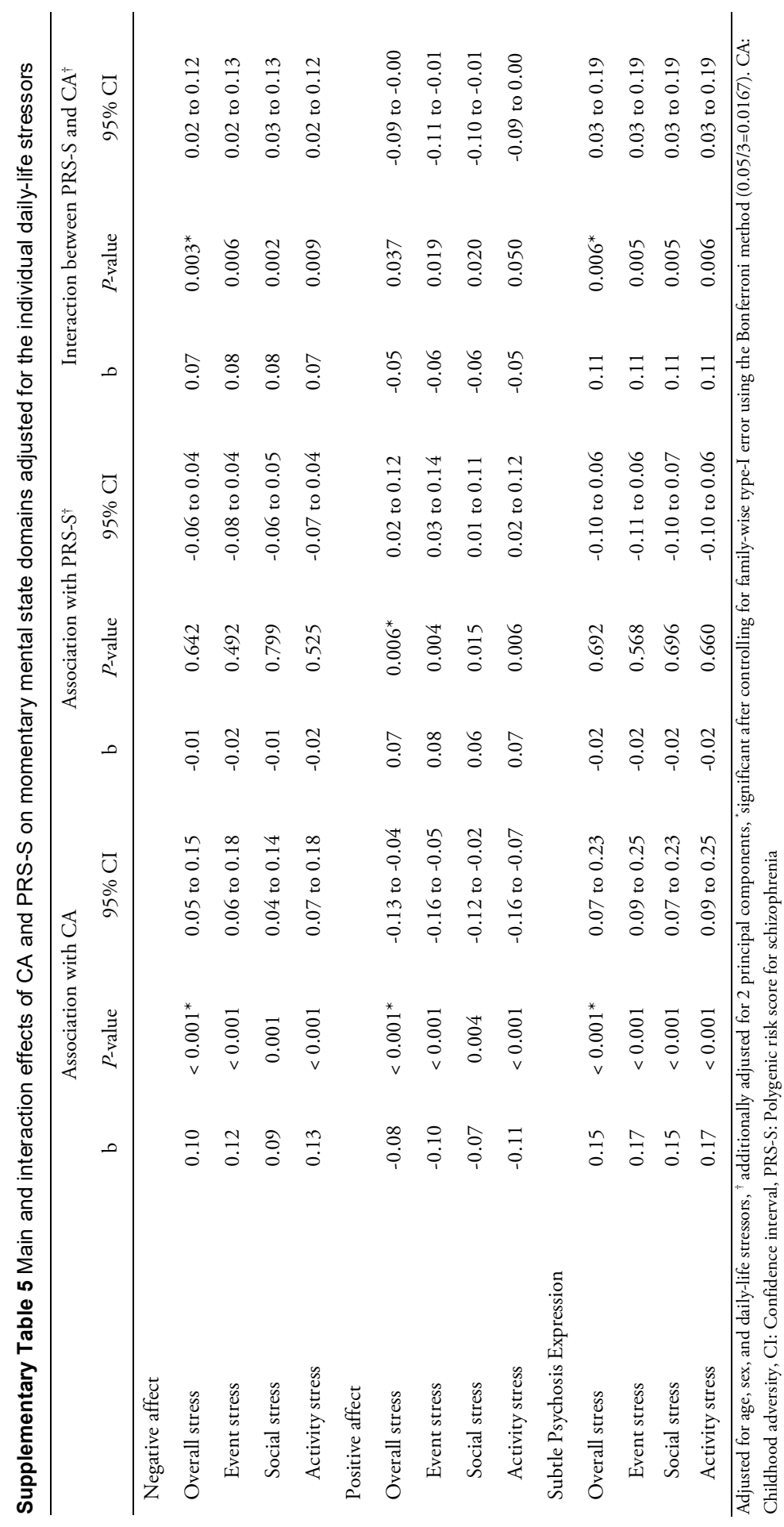


Supplementary Table 6 Main and interaction effects of daily-life stressors on momentary mental state domains

\begin{tabular}{|c|c|c|c|c|c|c|}
\hline & \multicolumn{3}{|c|}{$\begin{array}{l}\text { Association with } \\
\text { daily-life stressors }\end{array}$} & \multicolumn{3}{|c|}{$\begin{array}{c}\text { Interaction between PRS-S and daily-life } \\
\text { stressors }{ }^{\dagger}\end{array}$} \\
\hline & b & $P$-value & $95 \% \mathrm{CI}$ & $\mathrm{b}$ & $P$-value & $95 \% \mathrm{CI}$ \\
\hline \multicolumn{7}{|l|}{ Negative affect } \\
\hline Overall stress & 0.33 & $<0.001^{*}$ & 0.31 to 0.35 & -0.00 & 0.851 & -0.02 to 0.02 \\
\hline Event stress & 0.17 & $<0.001$ & 0.15 to 0.19 & -0.02 & 0.086 & -0.04 to 0.00 \\
\hline Social stress & 0.27 & $<0.001$ & 0.25 to 0.29 & -0.00 & 0.809 & -0.02 to 0.02 \\
\hline Activity stress & 0.24 & $<0.001$ & 0.22 to 0.26 & -0.01 & 0.522 & -0.03 to 0.01 \\
\hline \multicolumn{7}{|l|}{ Positive affect } \\
\hline Overall stress & -0.39 & $<0.001^{*}$ & -0.41 to -0.37 & 0.02 & 0.145 & -0.01 to 0.04 \\
\hline Event stress & -0.21 & $<0.001$ & -0.23 to -0.19 & 0.02 & 0.172 & -0.01 to 0.04 \\
\hline Social stress & -0.30 & $<0.001$ & -0.32 to -0.28 & 0.01 & 0.220 & -0.01 to 0.03 \\
\hline ...Activity stress & -0.29 & $<0.001$ & -0.31 to -0.27 & 0.01 & 0.148 & -0.01 to 0.03 \\
\hline \multicolumn{7}{|l|}{$\begin{array}{l}\text { Subtle psychosis } \\
\text { expression }\end{array}$} \\
\hline Overall stress & 0.21 & $<0.001^{*}$ & 0.19 to 0.23 & -0.01 & 0.464 & -0.03 to 0.01 \\
\hline Event stress & 0.10 & $<0.001$ & 0.08 to 0.12 & -0.01 & 0.216 & -0.03 to 0.01 \\
\hline Social stress & 0.17 & $<0.001$ & 0.15 to 0.19 & -0.00 & 0.676 & -0.03 to 0.02 \\
\hline Activity stress & 0.16 & $<0.001$ & 0.14 to 0.18 & -0.01 & 0.388 & -0.03 to 0.01 \\
\hline
\end{tabular}

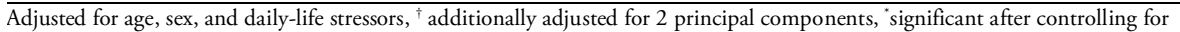
family-wise type-I error using the Bonferroni method $(0.05 / 3=0.0167)$.CA: Childhood adversity, CI: Confidence interval, PRSS: Polygenic risk score for schizophrenia 


\section{References}

1. Purcell S, Neale B, Todd-Brown K, et al. PLINK: a tool set for whole-genome association and population-based linkage analyses. Am J Hum Genet. 2007;81(3):559-575.

2. HapMap 3 [Internet] [cited 2018 Sep 16]:Available from:

https://www.sanger.ac.uk/resources/downloads/hu man/hapmap3.html.

3. Price AL, Patterson NJ, Plenge RM, Weinblatt ME, Shadick NA, Reich D. Principal components analysis corrects for stratification in genome-wide association studies. Nat Genet. 2006;38(8):904909.

4. Pardinas AF, Holmans P, Pocklington AJ, et al. Common schizophrenia alleles are enriched in mutation-intolerant genes and in regions under strong background selection. Nat Genet. 2018;50(3):381-389.

5. Price AL, Weale ME, Patterson N, et al. Longrange $L D$ can confound genome scans in admixed populations. Am J Hum Genet. 2008;83(1):132135 



\section{Abstract:}

Schizophrenia is a heritable complex phenotype associated with a background risk involving multiple common genetic variants of small effect and a multitude of environmental exposures. Early twin and family studies using proxy-genetic liability measures suggest geneenvironment interaction in the etiology of schizophrenia spectrum disorders, but the molecular evidence is scarce. Here, by analyzing the main and joint associations of polygenic risk score for schizophrenia (PRS-SCZ) and environmental exposures in 1,699 patients with a diagnosis of schizophrenia spectrum disorders and 1,542 unrelated controls with no lifetime history of a diagnosis of those disorders, we provide further evidence for gene-environment interaction in schizophrenia. Evidence was found for additive interaction of molecular genetic risk state for schizophrenia (binary mode of PRS-SCZ above $75 \%$ of the control distribution) with the presence of lifetime regular cannabis use and exposure to early-life adversities (sexual abuse, emotional abuse, emotional neglect, and bullying), but not with the presence of hearing impairment, season of birth (winter birth), and exposure to physical abuse or physical neglect in childhood. The sensitivity analyses replacing the a priori PRS-SCZ at $75 \%$ with alternative cut-points $(50 \%$ and $25 \%)$ confirmed the additive interaction. Our results suggest that the etiopathogenesis of schizophrenia involves genetic underpinnings that act by making individuals more sensitive to the effects of some environmental exposures.

Key words: Schizophrenia, psychosis, genetics, environment, gene-environment interaction, polygenic risk, childhood trauma, cannabis, bullying 
Schizophrenia is a complex phenotype characterized by reality distortion, cognitive alteration and negative symptoms. Although the prevalence of schizophrenia spectrum disorders is relatively low - approximately $0.47 \%$ for schizophrenia (the poor outcome fraction) and $3.0 \%$ for other clinical diagnoses of psychotic disorders ${ }^{1}-$ they account for a tremendous personal, economic and societal burden, with 218 disability adjusted life years (DALYs) per $100,000^{2}$, making schizophrenia the fifth leading cause of DALYs in the age group of 15-44 years. These figures indicate that there is an urgent need for breakthroughs in prevention, diagnosis and management of schizophrenia and related disorders, which can be achieved by increased understanding of etiopathology.

Decades of work consistently yielding high heritability estimates document the role of genetic background in the etiopathology of these disorders ${ }^{3,4}$. In agreement with findings from early family-based studies, recent results from the Danish nationwide registers confirm that the heritability estimates range from $73 \%$ for schizophrenia spectrum disorders to $79 \%$ for narrow schizophrenia diagnosis 5 .

Based on these findings from the field of quantitative genetic epidemiology, molecular genetics has emerged as arguably the most popular area of investigation in research targeting schizophrenia spectrum disorders. Easy and low-cost access to high-throughput techniques has increased genetic resolution. The Psychiatric Genomics Consortium ${ }^{6}$ was founded to achieve the power required to detect small effect sizes in a genome-wide association (GWA) analysis. The Schizophrenia Working Group of the Consortium identified 108 genome-wide significant $\operatorname{loci}^{7}$, and the number of novel genetic variants keeps growing as a function of sample size ${ }^{8}$. GWA findings, in line with the half-century-old polygenic theory of schizophrenia ${ }^{9}$, established that a large fraction of the genetic risk is explained by many common genetic variants with very small effects sizes.

However, the proportion of the genetic liability accounted for by single nucleotide polymorphisms (SNPs) detected in current GWA arrays represents only a fraction of the effect that was suggested by heritability estimates from twin studies. In other terms, there is a large "heritability gap" between twin and molecular genetics studies ${ }^{10}$. The most likely explanation for this gap is that part of the genetic effect documented by twin studies is contingent on environmental factors shared by individuals growing up in the same family ${ }^{10}$. The etiology of psychosis spectrum disorder is likely to involve genetic underpinnings that act by making individuals more sensitive to the effects of environmental exposures or by driving individuals to higher exposure rates ${ }^{11}$. 
In parallel to the growing knowledge base in genetics, environmental research into schizophrenia has produced consistent findings over years. Observational studies have identified various exposures associated with risk of psychosis spectrum disorder at different levels of evidence, with varying magnitude of the effect size estimates. These environmental risk factors include cannabis use, childhood adversities (e.g., sexual abuse, emotional neglect), peer-bullying, urban environment, proxies of social exclusion (e.g., ethnic minority, immigration, and hearing impairment), season of birth, and obstetric and pregnancy complications $^{12,13}$.

Although findings from empirical investigations relying on surrogates of genetic risk (i.e., familial history of schizophrenia) argue for a strong influence of environment in moderating genetic vulnerability ${ }^{11}$, operationalizing and translating these findings by using molecular candidate-gene approaches have been challenging tasks ${ }^{14}$.

The utilization of polygenic risk score (PRS) as a single metric of molecular genetic risk has considerably increased the power to detect associations with phenotypes as well as geneenvironment interactions. Currently, the PRS for schizophrenia (PRS-SCZ) of a subject can be estimated by summing the log odds ratios of individual SNPs multiplied by the number of risk alleles present at the corresponding loci ${ }^{15}$. PRS-SCZ has been shown to explain up to $7 \%$ of variation on the liability scale to schizophrenia, at least when using the latest release of the Psychiatric Genomics Consortium in patients with more chronic forms ${ }^{7}$.

We recently discussed the challenges of evaluating the role of environmental exposures in psychiatry and the need to use exposure-wide systematic approaches to separate genuine strong signals from selective reporting ${ }^{16}$. Guided by this, we aimed to analyze the main and joint associations of environmental exposures and PRS-SCZ in a cross-sectional sample that was specifically collected to test for gene-environment interactions in schizophrenia.

\section{Methods}

\section{Study population}

This case-control gene-environment interaction study used data from the Work-package 6 of the European Network of National Networks studying Gene-Environment Interactions in Schizophrenia (EUGEI) ${ }^{17}$ and the Genetic Risk and Outcome of Psychosis (GROUP) study within the EUGEI ${ }^{18}$. Data were collected between 2010 and 2015 in the Netherlands, Turkey, Spain and Serbia. 
Patients were diagnosed with schizophrenia spectrum disorders according to the DSM-IVTR (average duration of illness since age of first contact with mental health services $=9.9$ years). The diagnosis was later confirmed by the Operational Criteria Checklist for Psychotic and Affective Illness ${ }^{19}$ in the EUGEI, and the Schedules for Clinical Assessment in Neuropsychiatry $^{20}$ or the Comprehensive Assessment of Symptoms and History ${ }^{21}$ in the GROUP. Unrelated controls with no lifetime psychotic disorder were recruited from the same population as the cases. Exclusion criteria for all participants were a diagnosis of psychotic disorder due to another medical condition, a history of head injury with loss of consciousness, and an intelligence quotient $<70$.

A total of 1,866 patients and 1,583 healthy participants with genotype data available were included. As the predictive power of PRS-SCZ has not been established in people of nonwhite ethnic origin ${ }^{22}$, the present analyses were restricted to participants of Caucasian white ethnic origin. The final sample included 1,699 patients and 1,542 unrelated controls.

The projects were approved by the medical ethics committees of all participating sites and conducted in accordance with the Declaration of Helsinki. All respondents provided written informed consent. Participants below the age of 18 signed an assent; parent(s) also signed an informed consent.

To achieve high quality and homogeneity in clinical, experimental and environmental assessments, standardized instruments were administered by psychiatrists, psychologists or trained research assistants who completed mandatory on-site training sessions and online training modules, including interactive interview videos and self-assessment tools ${ }^{17,18}$. Both on-site and online training sessions were repeated annually to maintain high inter-rater reliability throughout the study enrollment period.

\section{Environmental exposures}

Within the limits of data availability, we sought to examine all the environmental exposures that have previously been associated with schizophrenia spectrum disorders.

Childhood adversity was assessed using the Childhood Trauma Questionnaire Short Form $(\mathrm{CTQ})^{23}$. This consists of 28 items, rated on a 5-point Likert scale, measuring five domains of maltreatment (emotional and physical neglect; emotional, physical and sexual abuse). The psychometric characteristics of the translated versions (Spanish, Turkish, Dutch and Serbian) of the CTQ have been comprehensively studied ${ }^{24-26}$. To dichotomize each childhood adversity domain $(0=$ "absent" and $1=$ "present"), consistent with previous work in the 
EUGEI $^{27}$, we used the following cut-off scores for each domain: $\geq 9$ for emotional abuse; $\geq 8$ for physical abuse; $\geq 6$ for sexual abuse; $\geq 10$ for emotional neglect; and $\geq 8$ for physical neglect.

Cannabis use was assessed by a modified version of the Cannabis Experiences Questionnaire ${ }^{28}$ in the EUGEI WP6 ( 0 = "none"; 1 = "only once or twice"; 2 = "a few times a year"; 3 = "a few times a month"; 4 = "once or more a week"; 5 = "everyday"), and by the L section of the Composite International Diagnostic Interview $(\mathrm{CIDI})^{29}$ in the GROUP ( 0 = "none"; $1=$ "less than weekly"; 2 = "weekly"; 3 = "daily"). Consistent with previous work ${ }^{30-32}$, a binary regular cannabis use variable was constructed by using the cut-off value of once or more per week during the lifetime period of most frequent use.

In accordance with previous studies investigating the association between season of birth and schizophrenia in the Northern hemisphere $\operatorname{sites}^{33}$, the high-risk birth period was defined based on the winter solstice (December-March), and a binary winter-birth exposure was constructed.

Hearing impairment was defined based on self-reported hearing impairment in the last 12 months $(0=$ "absent" and 1 = "present").

The history of bullying by peers (emotional, psychological or physical violence) before 17 years of age was assessed using the short version of the Retrospective Bullying Questionnaire $(\mathrm{RBQ})^{34,35}$, that measures the severity of the bullying experience: $0=$ "none"; 1 = "some (no physical injuries)"; 2 = "moderate (minor injuries or transient emotional reactions)"; 3 = "marked (severe and frequent physical or psychological harm)". Exposure to childhood bullying was dichotomized using $\geq 1$ as the cut-off point $(0=$ "absent" and $\geq 1$ = "present").

\section{Genetic data processing}

Samples of all individuals were genotyped at Cardiff University Institute of Psychological Medicine and Clinical Neurology, using custom Illumina HumanCoreExome-24 BeadChip genotyping arrays containing probes for 570038 genetic variants (Illumina, San Diego, CA). Genotype data were called using the GenomeStudio package and transferred into PLINK format for further analysis.

Quality control was conducted in PLINK v1.0736 or with custom Perl scripts. Variants with call rate $<98 \%$ were excluded from the dataset. Hardy-Weinberg equilibrium p-value was calculated separately in Turkish, Northern European and Southern European samples. 
Variants with Hardy-Weinberg equilibrium p-value $<1$ e- 6 in any of these three regions were excluded from the dataset. After quality control, 559505 variants remained.

Samples with call rate $<98 \%$ were excluded from the dataset. A linkage disequilibrium (LD) pruned set of variants was calculated using the --indep-pairwise command in PLINK (maximum $r^{2}=0.25$, window size $=500$ SNPs, window step size $=50$ SNPs) and used for further analyses. Homozygosity $\mathrm{F}$ values were calculated using the --het command in PLINK, and outlier samples $(\mathrm{F}<-0.11$ or $\mathrm{F}>0.15)$ were excluded. The genotypic sex of samples was calculated from X chromosome data using the --check-sex command in PLINK, and samples with different genotypic sex to their database sex were excluded.

Identity-by-descent values were calculated for the sample in PLINK. Samples with one or more siblings among the genotyped samples according to the database but no identified genotypic siblings (defined as PI-HAT $>0.35$ and $<0.65$ ) were excluded. After these were removed from consideration, samples with two or more database siblings in the database that were not supported by the genotypic data were also excluded.

After visually observing clustering of errors by genotyping chip, we decided to exclude chips with a high proportion of errors. All samples on chips with five or more sample exclusions due to heterozygosity or call rate (out of 12 possible samples) were excluded. All samples on chips with four or more sample exclusions due to sex or relative checks were also excluded, unless their identity was corroborated by concordance between database and genotype relatedness data with a sample on another chip.

Principal components were calculated in PLINK using LD pruned variants after combining the dataset with the Thousand Genomes reference. Due to the inherently multi-population nature of the dataset and the variety of possible analyses, no exclusions were made to the whole dataset based on this analysis. Population effects were corrected for separately in individual analyses.

After quality control, genotypes were imputed on the Michigan Imputation Server using the Haplotype Reference Consortium reference panel (version 1.1) and the programs Eagle for haplotype phasing and Minimac3 for imputation ${ }^{37,38}$. After imputation, variants with an imputation $r^{2}>0.6$, minor allele frequency $(\mathrm{MAF})>0.1 \%$ and call rate $>99 \%$ were retained (8277535 variants). Best-guess genotypes were generated from genotype probabilities using PLINK. 
PRS-SCZ was constructed using summary statistics from the Psychiatric Genomics Consortium genome-wide association study, excluding samples present in the GROUP data ${ }^{7}$. Clumping was performed in imputed best-guess genotypes for each dataset using PLINK (maximum $\mathrm{r}^{2}=0.2$, window size $=500 \mathrm{~kb}$, minimum $\mathrm{MAF}=10 \%$, minimum imputation information $(\mathrm{INFO})$ score $=0.7$ ), and variants within regions of long-range LD around the genome (including the human major histocompatibility complex) were excluded ${ }^{39}$. PRS-SCZ was then constructed from best-guess genotypes using PLINK at ten different $\mathrm{p}$-value thresholds $\left(1,0.5,0.3,0.2,0.1,0.05,0.01,1 \times 10^{-4}, 1 \times 10^{-6}, 5 \times 10^{-8}\right)$. Consistent with previous research in the field ${ }^{40-43}$, we used $\mathrm{p}=0.05$ for our primary analysis, as this threshold explained the most variation in the phenotype in the Psychiatric Genomics Consortium analysis ${ }^{7}$.

To be able to compare our estimates from the current sample with the previously reported estimates of the proportion of variance explained by PRS-SCZ, a logistic regression model was applied to test the association of PRS-SCZ with case-control status (adjusted for ancestry using the first ten principal components), and Nagelkerke's $\mathrm{R}^{2}$ was calculated. PRS-SCZ discriminated cases from controls (odds ratio, OR $=1.30 ; 95 \% \mathrm{CI}: 1.25-1.34 ; \mathrm{p}<0.001$; Nagelkerke's $\left.\mathrm{R}^{2}=0.15\right)$, after also controlling for age, sex and country $(\mathrm{OR}=1.30$; $95 \% \mathrm{CI}$ : 1.26-1.35; $\mathrm{p}<0.001$; Nagelkerke's $\mathrm{R}^{2}=0.20$ ).

PRS-SCZ was dichotomized using the quartile cut-off points based on the control distribution of PRS-SCZ within each country (to account for differences in PRS-SCZ between countries that may arise due to ethnic variation). The highest quartile (PRS-SCZ > $75 \%$ of the controls) was considered the binary genetic risk state for schizophrenia (hereafter: PRS-SCZ 75 ).

\section{Statistical analyses}

All analyses were carried out using the STATA version $15.0^{44}$. Random intercept multilevel logistic regression models, taking into account clustering of participants within countries, were applied to test the univariate associations of exposures and PRS-SCZ 75 with case status. For each exposure, gene-environment correlation was tested using multilevel logistic regression models in the control sample. To test gene-environment interaction, additive models were chosen over multiplicative models prior to data collection (EUGEI consortium meeting, December 14, 2013), because they provide superior representation of biological synergy ${ }^{45}$ and inform public health decisions within the sufficient cause framework ${ }^{46,47}$.

To test the joint effects of environmental exposures and genetic score, we entered the four states occasioned by the combination of each exposure and binary PRS-SCZ risk state (PRS- 
$\mathrm{SCZ}_{75}$ ) as independent variables (three dummy variables with no-risk state as the reference category), and case status as the dependent variable, in multilevel logistic regression models.

We tested for departure from additivity using the interaction contrast ratio, also called the relative excess risk due to interaction (RERI). The RERI is considered the standard measure for interaction on the additive scale in case-control studies ${ }^{48}$. The RERI was estimated as $\left(\mathrm{OR}_{\text {exposure\&PRS-SCZ75 }}-\mathrm{OR}_{\text {exposure }}-\mathrm{OR}_{\mathrm{PRS}-\mathrm{SCZ75}}+1\right)^{49}$. A RERI greater than zero was defined as a positive deviation from additivity, and considered significant when the $95 \%$ CI of RERI did not contain zero. Using the ORs derived from each model, the RERIs for each model were calculated using the delta method.

As a sensitivity measure, the alternative bootstrap percentile method ${ }^{50}(\mathrm{~N}=1,000$ bootstrap replications) was applied to estimate the bootstrapped 95\% CI for the RERI. All models were controlled for a priori covariates (age and sex), while models including PRS-SCZ 75 were additionally adjusted for ancestry, using the first ten principal components accommodating to the general recommendations. Following the extension to the STrengthening the Reporting of OBservational studies in Epidemiology (STROBE) reporting guidelines ${ }^{48}$, the interaction analyses were reported using a single reference category including the separate and joint effects of PRS-SCZ 75 and each exposure in strata of exposure and PRS-SCZ 75 .

The analyses were also conducted on imputed data, given missing observations in environmental exposure assessments. Under the assumption of missing at random, the multiple imputation chained equation mode $^{51}$ with 20 imputations restricted to in-range values was applied (relative efficiency ranging between $97 \%$ to $99 \%$ ). Imputed data were similar to observed values in the original dataset. All analyses were run on multiply imputed data, and estimates were pooled using Rubin's rules ${ }^{52}$.

To test the robustness of our findings, sensitivity analyses of binary genetic risk thresholds were conducted using the PRS-SCZ cut points at $50 \%$ and $25 \%$ of the controls. The nominal significance threshold was set at $\mathrm{p}=0.05$.

\section{Results}

Data concerning age, sex and environmental exposures in cases and controls are reported in Table 1. All exposures except winter birth were associated with case status, also after adjusting for age and sex. 
Table 1 Demographic variables and environmental exposures in cases and controls

\begin{tabular}{|c|c|c|c|c|}
\hline & Total & Controls & Cases & Missing rates \\
\hline Age (years, mean $\pm S D$ ) & $32.4 \pm 9.8$ & $33.4 \pm 10.6$ & $31.5 \pm 9.0$ & \\
\hline \multicolumn{5}{|l|}{ Sex } \\
\hline Male & $1,951(60.2 \%)$ & $762(49.4 \%)$ & $1,189(70.0 \%)$ & \\
\hline Female & $1,290(39.8 \%)$ & $780(50.6 \%)$ & $510(30.0 \%)$ & \\
\hline \multicolumn{5}{|l|}{ Cannabis use } \\
\hline No & $2,390(78.6 \%)$ & $1,366(91.2 \%)$ & $1,024(66.5 \%)$ & \multirow{2}{*}{$202(6.2 \%)$} \\
\hline Yes & $649(21.4 \%)$ & $132(8.8 \%)$ & $517(33.5 \%)$ & \\
\hline \multicolumn{5}{|l|}{ Bullying } \\
\hline No & 1,947 (72.3\%) & $1,101(83.7 \%)$ & 846 (61.4\%) & \multirow{2}{*}{$547(16.9 \%)$} \\
\hline Yes & 747 (27.7\%) & $215(16.3 \%)$ & $532(38.6 \%)$ & \\
\hline \multicolumn{5}{|l|}{ Emotional abuse } \\
\hline No & $2,019(73.0 \%)$ & $1,230(84.8 \%)$ & $789(60.0 \%)$ & \multirow{2}{*}{$475(14.7 \%)$} \\
\hline Yes & $747(27.0 \%)$ & $221(15.2 \%)$ & $526(40.0 \%)$ & \\
\hline \multicolumn{5}{|l|}{ Physical abuse } \\
\hline No & $2,477(88.7 \%)$ & $1,362(93.0 \%)$ & $1,115(84.0 \%)$ & \multirow{2}{*}{$450(13.9 \%)$} \\
\hline Yes & $314(11.3 \%)$ & $102(7.0 \%)$ & $212(16.0 \%)$ & \\
\hline \multicolumn{5}{|l|}{ Sexual abuse } \\
\hline No & $2,269(81.5 \%)$ & $1,309(90.1 \%)$ & $960(72.1 \%)$ & \multirow{2}{*}{$456(14.1 \%)$} \\
\hline Yes & $516(18.5 \%)$ & $144(9.9 \%)$ & $372(27.9 \%)$ & \\
\hline \multicolumn{5}{|l|}{ Emotional neglect } \\
\hline No & $1,254(45.3 \%)$ & $789(54.3 \%)$ & $465(35.4 \%)$ & \multirow{2}{*}{$473(14.6 \%)$} \\
\hline Yes & $1,514(54.7 \%)$ & $664(45.7 \%)$ & $850(64.6 \%)$ & \\
\hline \multicolumn{5}{|l|}{ Physical neglect } \\
\hline No & $1,804(64.8 \%)$ & $1039(71.3 \%)$ & $765(57.7 \%)$ & \multirow{2}{*}{$457(14.1 \%)$} \\
\hline Yes & $980(35.2 \%)$ & $419(28.7 \%)$ & $561(42.3 \%)$ & \\
\hline \multicolumn{5}{|l|}{ Winter birth } \\
\hline No & $1,989(63.2 \%)$ & $951(63.0 \%)$ & $1,038(63.4 \%)$ & \multirow{2}{*}{$94(2.9 \%)$} \\
\hline Yes & $1,158(36.8 \%)$ & $559(37.0 \%)$ & $599(36.6 \%)$ & \\
\hline \multicolumn{5}{|l|}{ Hearing impairment } \\
\hline No & $2,869(92.5 \%)$ & $1,437(95.6 \%)$ & $1,432(89.7 \%)$ & \multirow{2}{*}{$141(4.4 \%)$} \\
\hline Yes & $231(7.5 \%)$ & $66(4.4 \%)$ & $165(10.3 \%)$ & \\
\hline
\end{tabular}

Table 2 presents the unadjusted and adjusted ORs for PRS-SCZ 75 and each of the exposures associated with case status. Except for physical abuse, there was no evidence for gene-

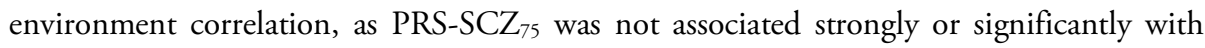
exposures in the control group (Table 3). Physical abuse was associated with PRS-SCZ 75 (adjusted OR $=1.84 ; 95 \% \mathrm{CI}: 1.19-2.84 ; \mathrm{p}=0.006$ ).

Table 4 reports the interactive effects of PRS-SCZ 75 and the exposures on the case status. There was evidence for additive interaction between $\mathrm{PRS}_{-} \mathrm{SCZ}_{75}$ and regular cannabis use $($ RERI $=5.60$; 95\% CI: 0.88-10.33; $\mathrm{p}=0.020)$, childhood bullying $(\mathrm{RERI}=2.76$; 95\% CI: 0.29-5.23; $\mathrm{p}=0.028$ ), emotional abuse (RERI $=5.52$; 95\% CI: 2.29-8.75; $\mathrm{p}<0.001$ ), sexual abuse (RERI $=7.61 ; 95 \% \mathrm{CI}: 2.05-13.17 ; \mathrm{p}=0.007$ ), and emotional neglect $(\mathrm{RERI}=2.46$; 95\% CI: 0.98-3.94; $\mathrm{p}=0.001$ ), respectively. Figure 1 visualizes the significant interaction 
effects on an additive scale. No evidence was found for significant additive interaction effects

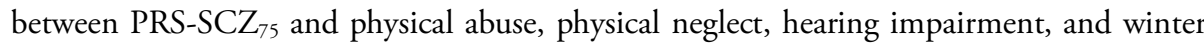
birth.

Table 2 Main effects of environmental and genetic risk on case-control status

\begin{tabular}{|c|c|c|c|c|}
\hline & \multicolumn{2}{|c|}{ Unadjusted main effects } & \multicolumn{2}{|c|}{ Adjusted main effects $^{2}$} \\
\hline & $\begin{array}{c}\text { Odds ratio } \\
(95 \% \mathrm{CI})\end{array}$ & $\mathrm{p}$ & $\begin{array}{c}\text { Odds ratio } \\
(95 \% \mathrm{CI})\end{array}$ & $\mathrm{p}$ \\
\hline Cannabis use & $4.85(3.89-6.05)$ & $<0.001$ & $3.96(3.16-4.97)$ & $<0.001$ \\
\hline Bullying & $3.01(2.48-3.65)$ & $<0.001$ & $3.06(2.50-3.74)$ & $<0.001$ \\
\hline Emotional abuse & $3.51(2.93-4.22)$ & $<0.001$ & $3.77(3.12-4.56)$ & $<0.001$ \\
\hline Physical abuse & $2.70(2.10-3.48)$ & $<0.001$ & $2.83(2.18-3.67)$ & $<0.001$ \\
\hline Sexual abuse & $3.66(2.96-4.53)$ & $<0.001$ & $4.11(3.30-5.13)$ & $<0.001$ \\
\hline Emotional neglect & $2.52(2.14-2.96)$ & $<0.001$ & $2.65(2.24-3.13)$ & $<0.001$ \\
\hline Physical neglect & $2.32(1.96-2.75)$ & $<0.001$ & $2.33(1.96-2.78)$ & $<0.001$ \\
\hline Winter birth & $1.06(0.92-1.23)$ & 0.423 & $1.05(0.91-1.23)$ & 0.495 \\
\hline Hearing impairment & $2.46(1.82-3.31)$ & $<0.001$ & $2.67(1.96-3.62)$ & $<0.001$ \\
\hline PRS-SCZ $75^{\mathrm{b}}$ & $2.91(2.48-3.40)$ & $<0.001$ & $2.85(2.43-3.35)$ & $<0.001$ \\
\hline
\end{tabular}

PRS-SCZ 75 - polygenic risk score for schizophrenia (75\% cut-point)

adjusted for sex and age, badjusted for ten principal components

Table 3 Gene-environment correlation between PRS-SCZ ${ }_{75}$ and environmental exposures

\begin{tabular}{lcccc}
\hline & $\begin{array}{c}\text { Unadjusted effects } \\
\text { Odds ratio } \\
(95 \% \mathrm{CI})\end{array}$ & $\mathrm{p}$ & $\begin{array}{c}\text { Adjusted effects }^{\mathrm{a}} \\
\text { Odds ratio } \\
(95 \% \mathrm{CI})\end{array}$ & $\mathrm{p}$ \\
\hline Cannabis use & $0.98(0.61-1.59)$ & 0.949 & $0.93(0.57-1.52)$ & 0.771 \\
Bullying & $1.27(0.86-1.86)$ & 0.228 & $1.28(0.87-1.89)$ & 0.210 \\
Emotional abuse & $1.13(0.80-1.58)$ & 0.493 & $1.13(0.81-1.59)$ & 0.476 \\
Physical abuse & $1.82(1.18-2.81)$ & 0.007 & $1.84(1.19-2.84)$ & 0.006 \\
Sexual abuse & $0.79(0.51-1.22)$ & 0.287 & $0.79(0.51-1.23)$ & 0.292 \\
Emotional neglect & $1.18(0.91-1.52)$ & 0.212 & $1.16(0.90-1.50)$ & 0.258 \\
Physical neglect & $1.18(0.89-1.56)$ & 0.246 & $1.19(0.90-1.58)$ & 0.219 \\
Winter birth & $1.13(0.88-1.45)$ & 0.338 & $1.13(0.88-1.45)$ & 0.332 \\
Hearing impairment & $1.13(0.63-2.02)$ & 0.693 & $1.18(0.65-2.13)$ & 0.592 \\
\hline
\end{tabular}

PRS-SCZ $_{75}$ - polygenic risk score for schizophrenia (75\% cut-point)

adjusted for sex, age and ten principal components

Analyses using the alternative bootstrap percentile method for estimating additive interactions yielded similar results (data not shown). The sensitivity analyses replacing the $a$ priori set PRS-SCZ 75 as the genetic risk in the models with the alternative cut-points of PRSSCZ (50\% and 25\%) confirmed that additive interaction was evident for regular cannabis use, childhood bullying, emotional abuse, sexual abuse, and emotional neglect across all PRSSCZ cut-points (data not shown). The results from the analyses performed in the imputed data were similar (Table 5). 


\section{Discussion}

In this study examining the main and joint associations of environmental exposures and genetic liability with schizophrenia spectrum disorder, evidence emerged for a positive additive interaction of genetic liability with regular cannabis use and childhood adversity domains (sexual abuse, emotional abuse, emotional neglect, and childhood bullying).

To the best of our knowledge, our study is the first to report that the sensitivity to adverse life events during childhood and exposure to cannabis is moderated by genetic risk state for schizophrenia (PRS-SCZ ${ }_{75}$ ). Put simply, the positive additive interaction between genetic liability and environmental exposure indicates synergy between gene and environment; that is, the combined influence of genetic liability and environmental exposure is larger than the sum of individual effects of each.

In line with previous findings, PRS-SCZ 75 discriminated cases from controls and all environmental exposures (except for winter birth) were associated with case status. However, no evidence for an additive interaction with PRS-SCZ 75 was observed for physical abuse, physical neglect, hearing impairment, or winter birth.

The proportion of variance explained by PRS-SCZ in our sample was comparable to previously reported estimates ${ }^{53}$ and the most recent findings from the Psychiatric Genomics Consortium $^{7}$. In this dataset, we strictly conformed to previous definitions of environmental exposures to improve reproducibility and allow comparability. In agreement with previous reports, our univariate analysis demonstrated that the exposures we tested were associated with case status to varying degrees, that were similar to meta-analytical estimates ${ }^{12,13}$.

By taking advantage of direct molecular measures of genetic risk, we provided further support for the putative role of gene-environment interaction in schizophrenia spectrum disorder that was observed in previous studies applying indirect genetic liability estimates derived from family-based (e.g., twin, relative) samples ${ }^{54}$. Our findings were corroborated by the results obtained from regression models using different genetic liability thresholds (PRS-SCZ cutoffs at $50 \%$ and $25 \%$ ) and analyses ran in imputed data.

The RERIs and 95\% CIs for emotional and sexual abuse were above 2, thereby suggesting a "mechanistic" interaction ${ }^{49}$, i.e., that there are individuals who would develop schizophrenia only when both genetic liability and environmental exposure (emotional or sexual abuse) are present, but would not develop schizophrenia when either genetic liability or environmental exposure is present alone. 
Table 4 Interaction of environmental exposures and PRS-SCZ 75 on case-control status

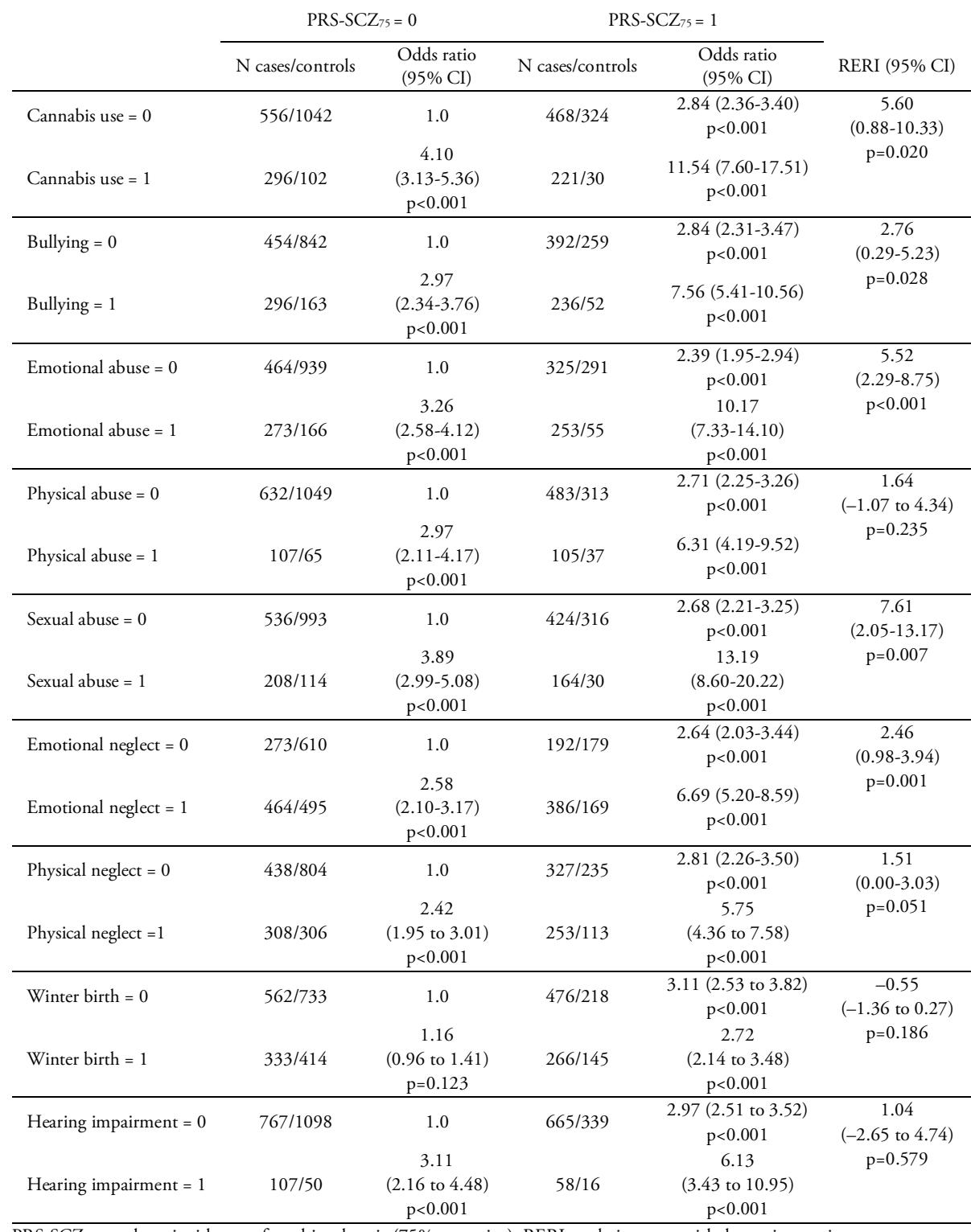

PRS-SCZ $_{75}$ - polygenic risk score for schizophrenia (75\% cut-point), RERI - relative excess risk due to interaction

Data adjusted for sex, age and ten principal components 
12

10

8

6

4

2

0

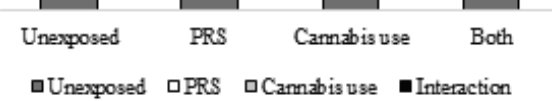

12

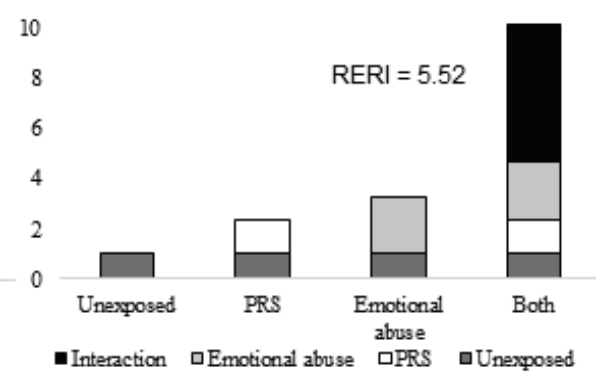

8

6

5

4

4
3

3
2

1

0

Both

口Unexposed aPRS 口Sexual abuse IInteraction

7

6

$\mathrm{RERI}=2.76$

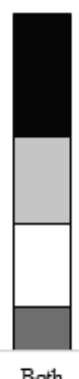

PRS

Bullying

Both

口Unexposed aPRS 口Bullying Interaction
Figure 1 Additive effects of cannabis use, emotional abuse, sexual abuse, emotional neglect and bullying on the association between the polygenic risk score for schizophrenia, $75 \%$ cut-point (PRS) and case-control status, adjusted for sex, age and ten principal components; RERI relative excess risk due to interaction 
Table 5 Additive interaction effects of PRS-SCZ $Z_{75}$ and the environmental exposures on case-control status in the imputed data

\begin{tabular}{lcccc}
\hline & $\begin{array}{c}\text { Main effects } \\
\text { Odds ratio } \\
(95 \% \mathrm{CI})\end{array}$ & $\mathrm{p}$ & $\begin{array}{c}\text { Interaction }^{\mathrm{b}} \\
\text { RERI } \\
(95 \% \mathrm{CI})\end{array}$ & $\mathrm{p}$ \\
\hline Cannabis use & $3.94(3.15-4.93)$ & $<0.001$ & $5.18(0.62-9.74)$ & 0.026 \\
Bullying & $2.88(2.36-3.51)$ & $<0.001$ & $2.88(0.63-5.13)$ & 0.012 \\
Emotional abuse & $3.49(2.88-4.24)$ & $<0.001$ & $5.11(2.10-8.13)$ & 0.001 \\
Physical abuse & $2.65(2.06-3.40)$ & $<0.001$ & $1.40(-1.10$ to 3.90$)$ & 0.272 \\
Sexual abuse & $3.74(3.00-4.66)$ & $<0.001$ & $6.84(1.77-11.92)$ & 0.008 \\
Emotional neglect & $2.51(2.14-2.95)$ & $<0.001$ & $2.37(0.90-3.84)$ & 0.002 \\
Physical neglect & $2.14(1.79-2.57)$ & $<0.001$ & $1.42(-0.05$ to 2.88$)$ & 0.058 \\
Winter birth & $1.06(0.91-1.23)$ & 0.485 & $-0.53(-1.36$ to 0.30$)$ & 0.209 \\
Hearing impairment & $2.68(1.97-3.66)$ & $<0.001$ & $1.24(-2.51$ to 5.00$)$ & 0.516 \\
\hline PRS-SCZ
\end{tabular}

PRS-SCZ $_{75}$ - polygenic risk score for schizophrenia (75\% cut-point), RERI - relative excess risk due to interaction

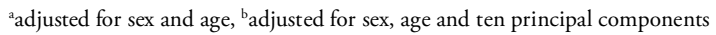

PRS-based approaches have recently gained traction in detecting gene-environment interaction. Previously, studies investigated the possible interaction between some genetic polymorphisms possibly linked to the putative biological mechanisms underlying psychosis and cannabis use or childhood adversity. Although SNPs (in various genes) for genetic moderation (e.g., AKT1, COMT, BDNF) were identified, these findings were inconsistent across samples ${ }^{55}$ and became secondary once the genome-wide approach took over the scene.

To date, a limited number of studies tested gene-environment interaction across the psychosis spectrum using PRS-SCZ. A pilot study of 80 patients with first-episode psychotic disorders and 110 controls investigating whether PRS-SCZ moderates the association between childhood adversities and psychosis, although yielding main effects of both PRS-SCZ and childhood adversities, was considerably underpowered to detect gene-environment interaction $^{56}$. A recent study demonstrated that intra-uterine environment moderates the association between PRS-SCZ and schizophrenia, and further revealed in the pathway analysis that genes involved in cellular stress response were the main drivers of the geneenvironment interaction ${ }^{57}$. In our recent study of a general population twin cohort, we found evidence for positive interaction effects between PRS-SCZ and exposure to childhood adversities to pleiotropically influence momentary emotional regulation and psychosis proneness $^{58}$. Further, a multimodal study combining genetics and imaging techniques reported that the association between PRS-SCZ and cortical maturation in young male adults is moderated by early-life exposure to cannabis ${ }^{59}$. Taken together, while the area of geneenvironment research is progressing rapidly toward a more replicable path informed by the use of GWA data, conclusive evidence has yet to emerge. 
There are various ways in which our findings can move forward gene-environment interaction research in the GWA era. First, they are useful in providing direction for future pre-registered confirmatory studies. Second, they may open up promising research lines for further exploration of gene-environment interactions in the biological context, such as using biologically-informative pathway scores instead of an aggregate genetic risk score for disease phenotype. These studies may help us investigate both hypotheses for biologically plausible pathways impacted by distinct exposures (e.g., hypoxia-ischemia pathway X obstetric complications and childhood adversities $\mathrm{X}$ hypothalamic-pituitary-adrenal axis) ${ }^{60,61}$, and putative common final pathways, such as the broad inflammatory pathway which may be influenced by many exposures cumulatively ${ }^{62}$.

However, there are important caveats: pathway scores may be less powerful than the overall polygenic scores for phenotypes, and there are almost endless options for selecting and constructing "putative" pathways. Therefore, gene-sets for pathways should be a priori defined and frozen at a central repository to avoid data-dredging. Further, study protocols for hypothesis-driven selective exposure and pathway analyses (e.g., regular cannabis use and endocannabinoid pathway) should ideally be either registered or, if this is not possible, agnostic data analyses should be followed through.

In our study, data were collected through extensive interviews by trained psychiatrists, psychologists and research assistants to specifically test the role of gene-environment interaction in schizophrenia. Further, our culturally and geographically diverse sample provided us with the advantage of observing variations in environmental exposures, which increases the power to detect interaction effects ${ }^{63}$.

However, some limitations should be acknowledged. First, the cross-sectional design informs only on temporal association and not causality. Nevertheless, cross-sectional analyses arguably remain an essential first step for identifying risk factors and pave the way for future longitudinal studies to investigate gene-environment interaction in evolutionary trajectories. Second, given the sample size and explorative nature of the study, we focused on main and interaction associations of previously established environmental factors and PRS-SCZ. However, the reality is much more complex than current statistical models can accommodate, involving dynamic interactions, causal and non-causal associations within the exposome (e.g., dense correlation matrix of environmental factors influenced by the timing, duration, severity and extent of repeated exposures over time ${ }^{16,64}$; the genome (e.g., epistasis, redundancy and pleiotropy) ${ }^{65}$; and the phenome (multidimensional syndromal diversity) ${ }^{66}$. Third, instead of the commonly-exercised selective reporting of one exposure at a time, we 
embraced a quasi-systematic approach to provide an overall picture of the gene-environment interactions findings from this dataset. However, we could not test some other known exposures (e.g., obstetric and pregnancy complications).

In conclusion, by using a molecular genetic risk measure, we have provided further evidence for the role of gene-environment interaction in schizophrenia. Our findings warrant further validation in pre-registered confirmatory research.

\section{Appendix}

GROUP investigators in EUGEI included: Behrooz Z. Alizadeh, Terese van Amelsvoort, Nico J. van Beveren, Richard Bruggeman, Wiepke Cahn, Lieuwe de Haan, Philippe Delespaul, Jurjen J. Luykx, Inez Myin-Germeys, Ruud van Winkel and Jim van Os

\section{Acknowledgments}

The EUGEI project was supported by the grant agreement HEALTH-F2-2010- 241909 from the European Community's Seventh Framework Programme. The authors are grateful to the patients and their families for participating in the project. They also thank all research personnel involved in the GROUP project, in particular J. van Baaren, E. Veermans, G. Driessen, T. Driesen, E. van't Hag and J. de Nijs. All the DNA samples from Turkey were provided by the Ankara University Brain Research Center Biobank, that was supported by Ankara Uni-versity Scientific Research Projects Coordination Unit (project no. 10A6055003, 2010). B.P.F. Rutten was funded by a VIDI award (no. 91718336) from the Netherlands Scientific Organization. S. Guloksuz, L.-K. Pries, B.P.F. Rutten and J. van Os contributed equally to this work. 


\section{References}

1. Perälä J, Suvisaari J, Saarni SI, et al. Lifetime prevalence of psychotic and bipolar I disorders in a general population. Archives of general psychiatry. 2007;64(1):19-28.

2. Murray CJ, Vos T, Lozano R, et al. Disabilityadjusted life years (DALYs) for 291 diseases and injuries in 21 regions, 1990-2010: a systematic analysis for the Global Burden of Disease Study 2010. The lancet. 2012;380(9859):2197-2223.

3. McGuffin P, Farmer AE, Gottesman II, Murray RM, Reveley AM. Twin concordance for operationally defined schizophrenia: Confirmation of familiality and heritability. Archives of General Psychiatry. 1984;41(6):541-545.

4. Cardno AG, Marshall EJ, Coid B, et al. Heritability estimates for psychotic disorders: the Maudsley twin psychosis series. Archives of general psychiatry. 1999;56(2):162-168.

5. Hilker R, Helenius D, Fagerlund B, et al. Heritability of schizophrenia and schizophrenia spectrum based on the nationwide Danish twin register. Biological psychiatry. 2018;83(6):492-498.

6. Sullivan PF, Agrawal A, Bulik CM, et al.

Psychiatric genomics: an update and an agenda. $A m$ J Psychiat. 2017;175(1):15-27.

7. Schizophrenia Working Group of the Psychiatric Genomics Consortium. Biological insights from 108 schizophrenia-associated genetic loci. Nature. 2014;511(7510):421-427.

8. Pardiñas AF, Holmans P, Pocklington AJ, et al. Common schizophrenia alleles are enriched in mutation-intolerant genes and in regions under strong background selection. Nature genetics. 2018;50(3):381.

9. Gottesman II, Shields J. A polygenic theory of schizophrenia. International Journal of Mental Health. 1972;1(1-2):107-115.

10. Uher R, Zwicker A. Etiology in psychiatry: embracing the reality of poly-gene-environmental causation of mental illness. World Psychiatry. 2017;16(2):121-129.

11. van Os J, Kenis G, Rutten BP. The environment and schizophrenia. Nature. 2010;468(7321):203.

12. Radua J, Ramella-Cravaro V, Ioannidis JP, et al. What causes psychosis? An umbrella review of risk and protective factors. World Psychiatry. 2018;17(1):49-66.

13. Belbasis L, Kohler CA, Stefanis N, et al. Risk factors and peripheral biomarkers for schizophrenia spectrum disorders: an umbrella review of metaanalyses. Acta Psychiatr Scand. 2018;137(2):88-97.
14. Duncan LE, Keller MC. A critical review of the first 10 years of candidate gene-by-environment interaction research in psychiatry. Am J Psychiat. 2011;168(10):1041-1049.

15. Wray NR, Lee SH, Mehta D, Vinkhuyzen AA, Dudbridge F, Middeldorp CM. Research review: polygenic methods and their application to psychiatric traits. Journal of Child Psychology and Psychiatry. 2014;55(10):1068-1087.

16. Guloksuz S, Rutten BPF, Pries LK, et al. The Complexities of Evaluating the Exposome in Psychiatry: A Data-Driven Illustration of Challenges and Some Propositions for Amendments. Schizophr Bull. 2018;44(6):11751179.

17. EUGEI, van Os J, Rutten BP, et al. Identifying gene-environment interactions in schizophrenia: contemporary challenges for integrated, large-scale investigations. Schizophr Bull. 2014;40(4):729736.

18. Korver N, Quee PJ, Boos HB, Simons CJ, de Haan L, Group Investigators. Genetic Risk and Outcome of Psychosis (GROUP), a multi site longitudinal cohort study focused on gene-environment interaction: objectives, sample characteristics, recruitment and assessment methods. International journal of methods in psychiatric research. 2012;21(3):205-221.

19. McGuffin P, Farmer A, Harvey I. A polydiagnostic application of operational criteria in studies of psychotic illness. Development and reliability of the OPCRIT system. Arch Gen Psychiatry. 1991;48(8):764-770.

20. Wing JK, Babor T, Brugha T, et al. Scan: Schedules fonr clinical assessment in neuropsychiatry. Archives of general psychiatry. 1990;47(6):589593.

21. Andreasen NC, Flaum M, Arndt S. The Comprehensive Assessment of Symptoms and History (CASH): an instrument for assessing diagnosis and psychopathology. Arch Gen Psychiatry. 1992;49(8):615-623.

22. Vassos E, Di Forti M, Coleman J, et al. An examination of polygenic score risk prediction in individuals with first-episode psychosis. Biological Psychiatry. 2017;81(6):470-477

23. Bernstein DP, Stein JA, Newcomb MD, et al. Development and validation of a brief screening version of the Childhood Trauma Questionnaire. Child Abuse Negl. 2003;27(2):169-190.

24. Şar V, Akyüz G, Kundakçı T, Kızıltan E, Doğan O. Childhood trauma, dissociation, and psychiatric 
comorbidity in patients with conversion disorder. Am J Psychiat. 2004;161(12):2271-2276.

25. Hernandez A, Gallardo-Pujol D, Pereda N, et al. Initial validation of the Spanish childhood trauma questionnaire-short form: factor structure, reliability and association with parenting. Journal of interpersonal violence. 2013;28(7):1498-1518.

26. Thombs BD, Bernstein DP, Lobbestael J, Arntz A. A validation study of the Dutch Childhood Trauma Questionnaire-Short Form: factor structure, reliability, and known-groups validity. Child Abuse Negl. 2009.

27. Kraan TC, Velthorst E, Themmen M, et al. Child maltreatment and clinical outcome in individuals at ultra-high risk for psychosis in the EU-GEI high risk study. Schizophrenia Bull. 2017;44(3):584592.

28. Barkus EJ, Stirling J, Hopkins RS, Lewis S. Cannabis-induced psychosis-like experiences are associated with high schizotypy. Psychopathology. 2006;39(4):175-178

29. Robins LN, Wing J, Wittchen HU, et al. The composite international diagnostic interview. An epidemiologic instrument suitable for use in conjunction with different diagnostic systems and in different cultures. Arch Gen Psychiatry. 1988;45(12):1069-1077.

30. van Winkel R, van Beveren NJ, Simons C, Genetic $\mathrm{R}$, Outcome of Psychosis I. AKT1 moderation of cannabis-induced cognitive alterations in psychotic disorder. Neuropsychopharmacology. 2011;36(12):2529-2537.

31. Pries LK, Guloksuz S, Ten Have M, et al. Evidence That Environmental and Familial Risks for Psychosis Additively Impact a Multidimensional Subthreshold Psychosis Syndrome. Schizophr Bull. 2018;44(4):710-719.

32. Radhakrishnan R, Guloksuz S, Ten Have M, et al. Interaction between environmental and familial affective risk impacts psychosis admixture in states of affective dysregulation. Psychol Med. 2019;49(11):1879-1889.

33. Davies G, Welham J, Chant D, Torrey EF, McGrath J. A systematic review and meta-analysis of Northern Hemisphere season of birth studies in schizophrenia. Schizophr Bull. 2003;29(3):587593.

34. Schäfer M, Korn S, Smith PK, et al. Lonely in the crowd: Recollections of bullying. 2004;22(3):379394.

35. Hunter SC, Mora-Merchan J, Ortega R. The longterm effects of coping strategy use in victims of bullying. Span J Psychol. 2004;7(1):3-12.
36. Purcell S, Neale B, Todd-Brown K, et al. PLINK: a tool set for whole-genome association and population-based linkage analyses. Am J Hum Genet. 2007;81(3):559-575.

37. Das S, Forer L, Schonherr S, et al. Next-generation genotype imputation service and methods. Nat Genet. 2016;48(10):1284-1287.

38. Loh PR, Danecek P, Palamara PF, et al. Referencebased phasing using the Haplotype Reference Consortium panel. Nat Genet. 2016;48(11):14431448.

39. Price AL, Weale ME, Patterson N, et al. Longrange $\mathrm{LD}$ can confound genome scans in admixed populations. Am J Hum Genet. 2008;83(1):132135; author reply 135-139.

40. Allardyce J, Leonenko G, Hamshere M, et al. Association between schizophrenia-related polygenic liability and the occurrence and level of mood-incongruent psychotic symptoms in bipolar disorder. JAMA psychiatry. 2018;75(1):28-35.

41. Escott-Price V, Smith DJ, Kendall K, et al. Polygenic risk for schizophrenia and season of birth within the UK Biobank cohort. Psychological medicine. 2018:1-6.

42. Sørensen HJ, Debost J-C, Agerbo E, et al. Polygenic Risk Scores, School Achievement, and Risk for Schizophrenia: A Danish Population-Based Study. Biological psychiatry. 2018.

43. van Os J, van der Steen Y, Islam MA, et al. Evidence that polygenic risk for psychotic disorder is expressed in the domain of neurodevelopment, emotion regulation and attribution of salience. Psychol Med. 2017;47(14):2421-2437.

44. StataCorp. STATA Statistical Software: Release 15. Texas: College Station; 2017.

45. Rothman KJ. The estimation of synergy or antagonism. American journal of epidemiology. 1976;103(5):506-511.

46. Kendler KS, Gardner CO. Interpretation of interactions: guide for the perplexed. The British Journal of Psychiatry. 2010;197(3):170-171.

47. Rothman KJ, Greenland S, Walker AM. Concepts of interaction. American journal of epidemiology. 1980;112(4):467-470.

48. Knol MJ, VanderWeele TJ. Recommendations for presenting analyses of effect modification and interaction. Int J Epidemiol. 2012;41(2):514-520.

49. VanderWeele TJ, Knol MJ. A tutorial on interaction. Epidemiologic Methods. 2014;3(1):3372.

50. Richardson DB, Kaufman JS. Estimation of the relative excess risk due to interaction and associated confidence bounds. American journal of epidemiology. 2009;169(6):756-760. 
51. Royston P, White IR. Multiple imputation by chained equations (MICE): implementation in Stata. J Stat Softw. 2011;45(4):1-20.

52. Rubin DB. Multiple imputation for nonresponse in surveys. Vol 81: John Wiley \& Sons; 2004.

53. Mistry S, Harrison JR, Smith DJ, Escott-Price V, Zammit $S$. The use of polygenic risk scores to identify phenotypes associated with genetic risk of schizophrenia: Systematic review. Schizophrenia Research. 2018;197:2-8.

54. van Os J, Rutten BP, Poulton R. Geneenvironment interactions in schizophrenia: review of epidemiological findings and future directions. Schizophr Bull. 2008;34(6):1066-1082.

55. Modinos G, Iyegbe C, Prata D, et al. Molecular genetic gene-environment studies using candidate genes in schizophrenia: a systematic review. Schizophrenia research. 2013;150(2-3):356-365.

56. Trotta A, Iyegbe C, Di Forti M, et al. Interplay between schizophrenia polygenic risk score and childhood adversity in first-presentation psychotic disorder: a pilot study. PloS one. 2016;11(9):e0163319.

57. Ursini G, Punzi G, Chen Q, et al. Convergence of placenta biology and genetic risk for schizophrenia. Nature medicine. 2018;24(6):792.

58. Pries LK, Klingenberg B, Menne-Lothmann C, et al. Polygenic liability for schizophrenia and childhood adversity influences daily-life emotion dysregulation and psychosis proneness. Acta Psychiatr Scand. 2020;141(5):465-475.

59. French L, Gray C, Leonard G, et al. Early cannabis use, polygenic risk score for schizophrenia and brain maturation in adolescence. JAMA psychiatry. 2015;72(10):1002-1011.

60. Schmidt-Kastner R, Van Os J, Esquivel G, Steinbusch H, Rutten B. An environmental analysis of genes associated with schizophrenia: hypoxia and vascular factors as interacting elements in the neurodevelopmental model. Molecular psychiatry. 2012;17(12):1194.

61. Binder EB. Understanding gene $x$ early adversity interactions: possibilities for insight in the biology of psychiatric disorders. Eur Arch Psychiatry Clin Neurosci. 2017;267(3):183-185.

62. Radhakrishnan R, Kaser M, Guloksuz S. The link between the immune system, environment, and psychosis. Schizophrenia bulletin. 2017;43(4):693697.

63. Ritz BR, Chatterjee N, Garcia-Closas M, et al. Lessons learned from past gene-environment interaction successes. American journal of epidemiology. 2017;186(7):778-786.

64. Guloksuz S, van Os J, Rutten BPF. The Exposome Paradigm and the Complexities of Environmental Research in Psychiatry. JAMA psychiatry. 2018;75(10):985-986

65. Pingault J-B, O'Reilly PF, Schoeler T, Ploubidis GB, Rijsdijk F, Dudbridge F. Using genetic data to strengthen causal inference in observational research. Nature Reviews Genetics. 2018:1.

66. Guloksuz S, van Os J. The slow death of the concept of schizophrenia and the painful birth of the psychosis spectrum. Psychol Med. 2018;48(2):229-244. 




\section{CHAPTER 8}

\section{ESTIMATING EXPOSOME SCORE}

FOR SCHIZOPHRENIA USING PREDICTIVE MODELING APPROACH IN TWO INDEPENDENT SAMPLES: THE RESULTS FROM THE EUGEI STUDY

Lotta-Katrin Pries, Agustin Lage Castellanos, Philippe Delespaul, Gunter Kenis, Jurjen J. Luykx, Bochao D. Lin, Alexander L. Richards, Berna Akdede,

Tolga Binbay, Vesile Altınyazar, Berna Yalınçetin, Güvem Gümüş-Akay, Burçin Cihan, Haldun Soygür, Halis Ulaş, Eylem Şahin Cankurtaran, Semra Ulusoy

Kaymak, Marina M. Mihaljevic, Sanja Andric Petrovic, Tijana Mirjanic, Miguel Bernardo, Bibiana Cabrera, Julio Bobes, Pilar A. Saiz, María Paz García-

Portilla, Julio Sanjuan, Eduardo J. Aguilar, José Luis Santos, Estela JiménezLópez, Manuel Arrojo, Angel Carracedo, Gonzalo López, Javier González-Peñas, Mara Parellada, Nadja P. Maric, Cem Atbaşoğlu, Alp Ucok, Köksal Alptekin, Meram Can Saka, Genetic Risk and Outcome of Psychosis (GROUP) investigators\#, Celso Arango, Michael O'Donovan, Bart P.F. Rutten*, Jim van Os*, Sinan Guloksuz*

Schizophrenia Bulletin (2019): 11(45):960-965

*Equally contributed \#The Genetic Risk and Outcome of Psychosis (GROUP) investigators in EUGEI are listed in the Appendix 


\section{Abstract}

Exposures constitute a dense network of environment: exposome. Here, we argue for embracing the exposome paradigm to investigate the sum of nongenetic "risk" and show how predictive modeling approaches can be used to construct an exposome score (ES; an aggregated score of exposures) for schizophrenia. The training dataset consisted of patients with schizophrenia and controls, whereas the independent validation dataset consisted of patients, their unaffected siblings, and controls. Binary exposures were cannabis use, hearing impairment, winter birth, bullying, and emotional, physical, and sexual abuse along with physical and emotional neglect. We applied logistic regression (LR), Gaussian Naive Bayes (GNB), the least absolute shrinkage and selection operator (LASSO), and Ridge penalized classification models to the training dataset. ESs, the sum of weighted exposures based on coefficients from each model, were calculated in the validation dataset. In addition, we estimated ES based on meta-analyses and a simple sum score of exposures. Accuracy, sensitivity, specificity, area under the receiver operating characteristic, and Nagelkerke's $R^{2}$ were compared. The $\mathrm{ES}_{\text {Meta-nalyses }}$ performed the worst, whereas the sum score and the $\mathrm{ES}_{\mathrm{GNB}}$ were worse than the $\mathrm{ES}_{\mathrm{LR}}$ that performed similar to the $\mathrm{ES}_{\mathrm{LASSO}}$ and $\mathrm{ES}_{\mathrm{RIDGE}}$. The $\mathrm{ES}_{\mathrm{LR}}$ distinguished patients from controls (odds ratio $[\mathrm{OR}]=1.94, P<0.001$ ), patients from siblings $(\mathrm{OR}=1.58, P<0.001)$, and siblings from controls $(\mathrm{OR}=1.21, P=0.001)$. An increase in $\mathrm{ES}_{\mathrm{LR}}$ was associated with a gradient increase of schizophrenia risk. In reference to the remaining fractions, the $\mathrm{ES}_{\mathrm{LR}}$ at top $30 \%, 20 \%$, and $10 \%$ of the control distribution yielded ORs of $3.72,3.74$, and 4.77 , respectively. Our findings demonstrate that predictive modeling approaches can be harnessed to evaluate the exposome.

Key words: Schizophrenia, psychosis, predictive modeling, machine learning, risk score, environment, childhood trauma, cannabis, winter birth, hearing impairment 


\section{Introduction}

Several environmental exposures have been associated with psychosis spectrum disorder ${ }^{1,2}$. Knowledge on this association has thus far been deduced from hypothesis-driven selective one-exposure to one-outcome studies, akin to the candidate-gene approach ${ }^{3}$. However, each exposure constitutes a fraction of a dense network of exposures: the exposome $e^{4}$. Here, we argue for embracing the exposome paradigm to investigate the sum of the nongenetic "risk" and show how a predictive modeling approach can be used to construct an exposome score (ES) for schizophrenia, a single metric of aggregated environmental load similar to polygenic risk score ${ }^{5}$.

\section{Approach}

Guided by the predictive modeling methods for constructing cumulative environmental exposure scores ${ }^{6,7}$, we used 2 independent datasets to, first, build a predictive model in the training dataset (the Work-package 6 of the European Network of National Networks studying Gene-Environment Interactions in Schizophrenia $[\mathrm{EUGEI}]^{2}$ ) and, second, construct and test the ES in the validation dataset (the Genetic Risk and Outcome of Psychosis [GROUP] study ${ }^{8}$ ). We examined the following widely evaluated environmental factors that we also recently investigated individually within the context of gene-environment interaction': hearing impairment, winter birth, cannabis use, and childhood adversities (bullying, emotional, physical and sexual abuse along with emotional and physical neglect) ${ }^{10}$. Our analysis was limited to the environmental exposures that were reliably measured and equally available in both datasets. These environmental factors were defined according to previous studies ${ }^{9}$. The detailed description of each environmental exposure is provided in the supplementary file. We used 4 prediction models to determine to what degree cumulative environmental exposure contributes to the liability for schizophrenia in a case-control design. Logistic regression (LR), Gaussian Naive Bayes (GNB), and penalized logistic regression (least absolute shrinkage and selection operator [LASSO] and Ridge) were applied to data with complete information on environmental exposures. The description of the models and the distribution of exposures are provided in the supplementary file. For each model, the dependent variable was the binary case-control status, whereas binary environmental exposures were features (independent variables). First, we estimated coefficients of binary exposures in the training dataset including 1241 healthy controls and 747 patients with a diagnosis of schizophrenia spectrum disorders. Second, we calculated the weighted sum of the exposures according to each predictive model in an independent validation dataset with 323 healthy controls, 463 patients with a diagnosis of schizophrenia spectrum disorders, and 542 unaffected siblings of the patients. To compare the performance of ES from each model, we also generated an environmental sum score by simply adding each binary exposure per 
individual as $0=$ absent and $1=$ present (the sum score is ranging from 0 to 9 ) and a cumulative environmental score weighted by the meta-analytical estimates for each exposure ${ }^{11-14}$, conforming to a previous study ${ }^{15}$. Finally, we tested the performance of ESs derived from each model by applying logistic regression in a case-control design in the independent validation dataset by evaluating the area under the receiver operating characteristic (ROC), accuracy (ACC), sensitivity, specificity, and Nagelkerke's pseudo $R^{2}$. In this regard, we prioritized models with better sensitivity than specificity as our main concern was to avoid misclassifying individuals diagnosed with schizophrenia.

\section{Prediction in the training dataset}

The coefficients of individual models (see figure 1a and supplementary table S2) indicate that cannabis use (coefficients ranging from 1.31 to 1.53), hearing impairment (coefficients: 1.10-1.19), and bullying (coefficients: 1.30-1.57) received the highest weights in the training dataset. The lowest weight was attributed to winter birth with coefficients between 0.01 and 0.06. In comparison with the GNB model, which assumes independence between predictors, the LR, Ridge, and LASSO models yielded lower weights for emotional abuse, sexual abuse, emotional neglect, physical neglect, and bullying. Further, although physical abuse was a strong positive predictor in the GNB model, its predictive value was lost and even yielded a negative weight when using predictive model approaches that account for dependence between the predictors. This is in line with evidence that exposures are weakly to moderately correlated with each other ${ }^{3,16-18}$. Consequently, coefficients are overestimated when independence is assumed.

\section{Constructing and testing the performance of exposome score in an independent dataset}

The ROC was used to estimate the performance of the calculated ESs in predicting the casecontrol status in the validation dataset (figure $1 \mathrm{~b}$ and supplementary table S3). The ES based on meta-analytical estimates (the $\mathrm{ES}_{\text {Meta-analyses }}$ ), $\mathrm{ES}_{\mathrm{GNB}}$, and the environmental sum score yielded the lowest ROC, 0.69, 0.71 and 0.71, respectively, whereas all other ESs $\left(E S_{\mathrm{LR}}\right.$, $\mathrm{ES}_{\mathrm{RIDGE}}$, and $\mathrm{ES}_{\mathrm{LASSO}}$ ) had ROC ranging from 0.73 to 0.74 . With a chance level of 0.5 (as patients and controls were in balance in the training sample, see supplementary file), all ESs indicated an ACC above chance level (ACC: 0.62-0.68) with specificity between 0.42 and 0.72 , and sensitivity between 0.56 to 0.86 . Compared to the $E S_{\mathrm{LR}}, \mathrm{ES}_{\mathrm{RIDGE}}$, and $\mathrm{ES}_{\mathrm{LASSO}}$, the ESs derived from the models assuming independence between exposures (ES $\mathrm{GNB}_{\mathrm{G}}$, environmental sum score, and $\left.\mathrm{ES}_{\text {Meta-analyses }}\right)$ performed worse on sensitivity and had more false negatives as they incorrectly classified patients as healthy. Given that our priority was reducing false negatives rather than reducing false positives and that the $\mathrm{ES}_{\mathrm{LR}}, \mathrm{ES}_{\mathrm{RIDGE}}$, and 
$\mathrm{ES}_{\text {LASSO }}$ performed similarly well (figure $1 \mathrm{~b}$ and supplementary table S3), we reported further analyses with the $\mathrm{ES}_{\mathrm{LR}}$, which was constructed on the basis of a widely available and commonly used statistical model, logistic regression.

a)

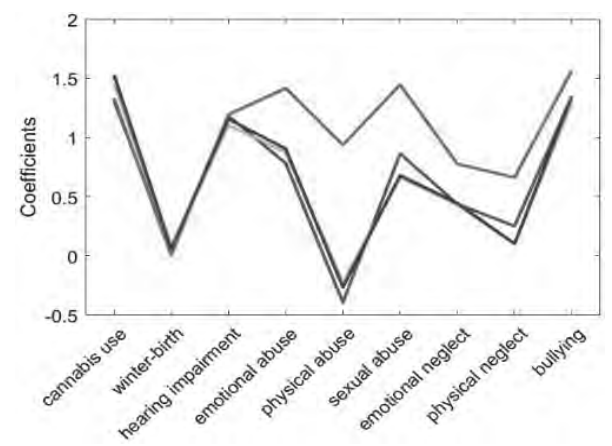

c)

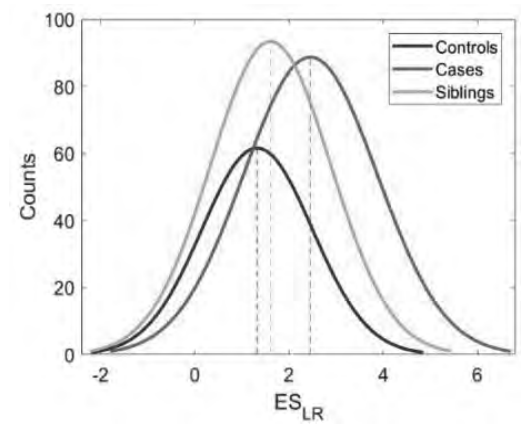

b)

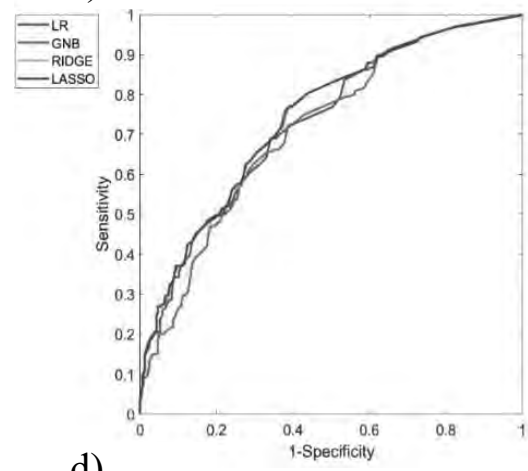

d)

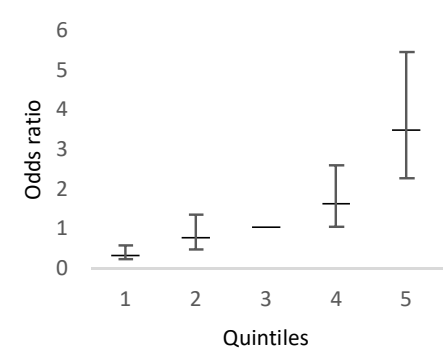

Figure 1 a) Coefficients profile for each exposure derived from different classification methods in the training dataset, GNB: Gaussian Naive Bayes, LR: logistic regression, b) The area under the receiver operating characteristic (ROC) for the different exposome scores in the validation dataset, c) The histogram of the $\mathrm{ES}_{\mathrm{LR}}$ (exposome score based on logistic regression) for patients, siblings and controls in the validation dataset. For visualization, a gaussian distribution was fit to histogram counts by adjusting mean and standard deviations, d) The risk strata plot of the $\mathrm{ES}_{\mathrm{LR}}$ on case-control status: The $\mathrm{ES}_{\mathrm{LR}}$ was divided into 5 quintiles (XAxis) of the control distribution and logistic regression was applied to case-control status as the dependent variable. The third quintile includes the median and was used as reference. The Y-Axis represents odds ratios and the error bars show confidence intervals.

To examine whether the $\mathrm{ES}_{\mathrm{LR}}$ reflects schizophrenia liability in the validation dataset, we evaluated the $\mathrm{ES}_{\mathrm{LR}}$ in patients, siblings, and controls (see figure 1c for an illustration and supplementary table $\mathrm{S} 4$ for the other models). The $\mathrm{ES}_{\mathrm{LR}}$ discriminated patients from controls (odds ratio $[\mathrm{OR}]=1.94,95 \%$ confidence interval $[\mathrm{CI}]=1.71-2.20, P<0.001$, Nagelkerke's pseudo $\left.R^{2}=0.21\right)$, also after adjusting for age and sex $(\mathrm{OR}=1.87,95 \% \mathrm{CI}=1.64-2.14$, $P<0.001)$ in the validation dataset. Similarly, logistic regression analysis showed higher $\mathrm{ES}_{\mathrm{LR}}$ in patients compared to siblings $(\mathrm{OR}=1.58,95 \% \mathrm{CI}=1.43-1.74, P<0.001$; adjusted for age and sex: $\mathrm{OR}=1.55,95 \% \mathrm{CI}=1.40-1.72, P<0.001)$ and in siblings compared to controls 
$(\mathrm{OR}=1.21,95 \% \mathrm{CI}=1.08-1.36, P=0.001$; adjusted for age and sex: $\mathrm{OR}=1.23,95 \% \mathrm{CI}=$ $1.09-1.38, P<0.001)$.

To visually represent the risk stratification properties of the $\mathrm{ES}_{\mathrm{LR}}$, we categorized the $\mathrm{ES}_{\mathrm{LR}}$ using the quintiles of the control distribution and measured the case-control ORs using the middle quintile (median $E S_{\mathrm{LR}}$ ) as the reference. With an increase of the $E S_{\mathrm{LR}}$, we noticed a gradient increase in the risk for schizophrenia. In comparison with the median, the fifth quintile had a higher $\mathrm{OR}(\mathrm{OR}=3.47 ; 95 \% \mathrm{CI}=2.22-5.41 ; P<0.001$ and age- and sexadjusted $\mathrm{OR}=3.78 ; 95 \% \mathrm{CI}=2.34-6.09 ; \mathrm{P}<0.001)$ and the first quintile had a lower OR $(\mathrm{OR}=0.30 ; 95 \% \mathrm{CI}=0.17-0.53 ; P<0.001$ and age- and sex-adjusted $\mathrm{OR}=0.34 ; 95 \% \mathrm{CI}$ $=0.19-0.62 ; P<0.001$; figure $1 \mathrm{c})$. We then dichotomized the $\mathrm{ES}_{\mathrm{LR}}$ with cut-off points at $70 \%, 80 \%$, and $90 \%$ of the control distribution. Comparing the top and the bottom part translated to ORs of 3.81, 3.96, and 5.11 (age- and sex-adjusted ORs of 3.72, 3.74, and 4.77) for $70 \%, 80 \%$, and $90 \%$ of the distribution, respectively (supplementary table S5).

\section{Discussion}

For the first time, we applied a predictive modeling approach to construct the ES for schizophrenia by leveraging 2 large independent datasets (training and validation data) with similar assessment protocols for environmental exposures. Our findings suggest that predictive modeling can be used to estimate environmental loading of a range of exposures. We found that the $E S_{\mathrm{LR}}, \mathrm{ES}_{\mathrm{RIDGE}}$, and $\mathrm{ES}_{\mathrm{LASSO}}$ performed similarly well, whereas the ESs derived from the models assuming independence performed worse. Of the $\mathrm{ES}_{\mathrm{GNB}}, \mathrm{ES}_{\mathrm{Meta}}$ analyses, and the simple summation of exposures, the $\mathrm{ES}_{\text {Meta-analyses, }}$, relying on the external sources for extracting estimates for environmental exposures, showed the worst performance.

The low performance of the ES driven by meta-analyses might be related to the fact that meta-analytical estimates are derived from different studies that use different assessments, different definitions, and different cut-off points for exposures in different study populations ${ }^{3}$, which might not be completely compatible with the dataset at hand. The availability of similar training and validation datasets plays a major role in prediction power-for instance, the predictive performance of polygenic scores for schizophrenia is considerably lower in non-Caucasian ancestry samples ${ }^{19}$. Therefore, a similar situation exists in estimating genetic liability, which, however, has the advantage of using more concrete, uniformly measured genetic variation for prediction in comparison to environmental assessment. Generating a uniform "environmental risk score" is even more challenging. For instance, cannabis use could be scored positive if participants smoke daily, or at least weekly, or at least monthly for lifetime use or exposure during adolescence, while childhood 
adversities could similarly be measured by various methods. Therefore, as weights are determined by how strict or lenient the cut-off points are, it is likely that the inconsistency between sampling and measurement strategies would introduce bias. Further, when individual coefficients from meta-analyses are used for a weighted environmental score, correlations between exposures are ignored, and weights may be overestimated ${ }^{3}$. In line with this, we also show that GNB, which assumes independence between predictors, produces higher weights for exposures than the other data-driven models.

Similar to current results, previous studies show that more contemporary algorithms do not necessarily translate into superior performance over logistic regression for clinical prediction modeling ${ }^{20,21}$. However, it should be noted that our analysis did not involve a complex data structure with many predictors. Penalized classification models might have led to performance improvement if more complex structures had to be considered (e.g., increasing the number of predictors and adding pairwise interactions). Researchers likewise need to be cautious about overfitting models and be aware that, if environmental exposures are correlated, the initial simple model with a few predictors will show the highest portion of improvement. However, each sequentially added predictor would result in less and less improvement in model performance ${ }^{21}$.

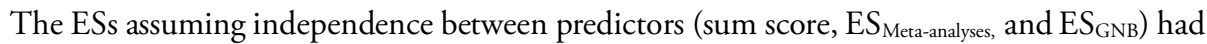
lower sensitivity than the rest. The $\mathrm{ES}_{\text {Meta-analyses }}$ indicated the lowest sensitivity (56\%). The sensitivity of an environmental score derived from meta-analytical estimates in a previous study was even lower, only around $7-9 \%{ }^{15}$. In other words, predictive models that do not assume independence between exposures may more accurately classify patients as positive by decreasing false negatives. The ESs from the models assuming independence, however, had higher specificity and were better in decreasing false positives. As our main concern was to avoid misclassifying individuals diagnosed with schizophrenia, we chose sensitivity over specificity. Further, if more environmental exposures were to be included in the ES, thus introducing more correlation, the models not assuming independence between predictors $\left(E S_{\mathrm{LR}}, E S_{\mathrm{RIDGE}}\right.$ and $\mathrm{ES}_{\mathrm{LASSO}}$ ) would perform increasingly better than the models assuming independence (sum score, $\mathrm{ES}_{\text {Meta-analyses, }}$ and $\mathrm{ES}_{\mathrm{GNB}}$ ).

The $\mathrm{ES}_{\mathrm{LR}}$, generated using an easily accessible method (logistic regression), achieved similar performance results compared with the $\mathrm{ES}_{\mathrm{RIDGE}}$ and $\mathrm{ES}_{\mathrm{LASSO}}$. We used the $\mathrm{ES}_{\mathrm{LR}}$ to further explore the characteristics of the ES in the follow-up analyses. In general, patients had higher $\mathrm{ES}_{\mathrm{LR}}$ than both controls and siblings, whereas siblings had higher $\mathrm{ES}_{\mathrm{LR}}$ than controls. The $\mathrm{ES}_{\mathrm{LR}}$ explained more variance $\left(\right.$ Nagelkerke $\left.R^{2}=0.21\right)$ than the $\mathrm{ES}_{\text {Meta-analyses }}\left(\right.$ Nagelkerke $R^{2}=$ 
0.13). In accordance with our previous findings showing an additive effect for environmental factors $^{22,23}$, our results indicate that the $\mathrm{ES}_{\mathrm{LR}}$ shows a dose-response effect: the odds of schizophrenia increase as a function of the $\mathrm{ES}_{\mathrm{LR}}$. Eventually, an individual with $\mathrm{ES}_{\mathrm{LR}}$ in the top $10 \%$ of the control distribution was around 5 times more likely to have schizophrenia compared to an individual below that cutoff.

\section{Limitations of exposome score}

Our analysis was limited to the environmental exposures that were reliably measurable and equally available in both datasets. The ES can be extended to include other environmental exposures (e.g., obstetric and pregnancy complications and urban environment). We included winter birth as an exposure in the current analyses as previous studies suggest an association between winter birth and psychosis ${ }^{14}$. However, summer birth was also previously associated with deficit schizophrenia and might therefore be evaluated as an exposure as well ${ }^{24,25}$. Considering evidence showing that common environmental factors (e.g., childhood adversity) are not specific to the psychosis phenotype, but instead are more generally related to psychopathology $y^{26,27}$, the ES would likely (to a degree) be associated with other mental disorders in mixed samples. Therefore, a low discriminant capacity for the ES should be anticipated. Given the nature of observational studies, causality claims should be avoided. Finally, it should be noted that although aggregating exposures leads to an increase in the predictive power and may be particularly beneficial in exploring shared mechanisms, the inherent heterogeneity of a single score may lead to information loss and biological imprecision. Considering the reasons described earlier, we have avoided using the term "risk" and opted for a neutral alternative: exposome score.

\section{Conclusion}

Our findings demonstrate that predictive modeling approaches can be harnessed to evaluate the exposome. In the future, we aim to explore models by including more exposures as well as interaction terms and test the predictive power of the ES in epidemiologically representative general population cohorts.

\section{Funding}

The EUGEI project was supported by the grant agreement HEALTH-F2-2010-241909 from the European Community's Seventh Framework Programme. The authors are grateful to the patients and their families for participating in the project. They also thank all research personnel involved in the GROUP project, in particular J. van Baaren, E. Veermans, 
G. Driessen, T. Driesen, E. van't Hag and J. de Nijs. Bart PF Rutten was funded by a VIDI award number 91718336 from the Netherlands Scientific Organisation.

\section{Appendix}

GROUP-EUGEI investigators are: Behrooz Z. Alizadeh ${ }^{\mathrm{a}}$, Therese van Amelsvoort ${ }^{\mathrm{b}}$, Richard Bruggeman $^{\mathrm{a}}$, Wiepke Cahn ${ }^{\mathrm{c}, \mathrm{d}}$, Lieuwe de Haan ${ }^{\mathrm{e}}$, Jurjen J. Luykx ${ }^{\mathrm{c}, \mathrm{f,g}}$, Ruud van Winkel ${ }^{\mathrm{b}, \mathrm{h}}$, Bart P.F. Rutten ${ }^{\mathrm{b}}$, Jim van $\mathrm{Os}^{\mathrm{c}, \mathrm{b}, \mathrm{i}}$,

${ }^{a}$ University of Groningen, University Medical Center Groningen, University Center for Psychiatry, Rob Giel Research center, Groningen, The Netherlands;

b Maastricht University Medical Center, Department of Psychiatry and Neuropsychology, School for Mental Health and Neuroscience, Maastricht, The Netherlands;

c Department of Psychiatry, UMC Utrecht Brain Center, University Medical Center Utrecht, Utrecht University, Utrecht, the Netherlands;

d Altrecht, General Menthal Health Care, Utrecht, The Netherlands;

${ }^{\mathrm{e}}$ Amsterdam UMC, University of Amsterdam, Department of Psychiatry, Amsterdam, The Netherlands;

${ }^{f}$ Department of Translational Neuroscience, UMC Utrecht Brain Center, University Medical Center Utrecht, Utrecht University, Utrecht, The Netherlands;

${ }^{\mathrm{g}}$ GGNet Mental Health, Apeldoorn, The Netherlands;

${ }^{\text {h }}$ KU Leuven, Department of Neuroscience, Research Group Psychiatry, Leuven, Belgium King's;

i College London, King's Health Partners, Department of Psychosis Studies, Institute of Psychiatry, London, United Kingdom 


\section{References}

1. van Os J, Kenis G, Rutten BP. The environment and schizophrenia. Nature. 2010;468(7321):203212.

2. EUGEI. Identifying gene-environment interactions in schizophrenia: contemporary challenges for integrated, large-scale investigations. Schizophrenia bulletin. 2014;40(4):729-736.

3. Guloksuz S, Rutten BPF, Pries LK, et al. The Complexities of Evaluating the Exposome in Psychiatry: A Data-Driven Illustration of Challenges and Some Propositions for Amendments. Schizophr Bull. 2018;44(6):11751179.

4. Guloksuz S, van Os J, Rutten BPF. The Exposome Paradigm and the Complexities of Environmental Research in Psychiatry. JAMA psychiatry. 2018;75(10):985-986.

5. Schizophrenia Working Group of the Psychiatric Genomics Consortium. Biological insights from 108 schizophrenia-associated genetic loci. Nature. 2014;511(7510):421-427.

6. Oulhote Y, Bind M-A, Coull B, Patel CJ, Grandjean P. Combining Ensemble Learning Techniques and G-Computation to Investigate Chemical Mixtures in Environmental Epidemiology Studies. 2017:147413.

https://www.biorxiv.org/content/biorxiv/early/2017 /06/30/147413.full.pdf.

7. Park SK, Zhao Z, Mukherjee B. Construction of environmental risk score beyond standard linear models using machine learning methods: application to metal mixtures, oxidative stress and cardiovascular disease in NHANES. Environmental Health. 2017;16(1):102.

8. Korver N, Quee PJ, Boos HB, Simons CJ, de Haan L, Investigators G. Genetic Risk and Outcome of Psychosis (GROUP), a multi site longitudinal cohort study focused on gene-environment interaction: objectives, sample characteristics, recruitment and assessment methods. Int J Methods Psychiatr Res. 2012;21(3):205-221.

9. Guloksuz S, Pries L-K, Delespaul P, et al. Examining the independent and joint effects of molecular genetic liability and environmental exposures in schizophrenia: results from the EUGEI study. World Psychiatry. In press.

10. Belbasis L, Kohler CA, Stefanis N, et al. Risk factors and peripheral biomarkers for schizophrenia spectrum disorders: an umbrella review of metaanalyses. Acta Psychiatr Scand. 2018;137(2):88-97.

11. Linszen MM, Brouwer RM, Heringa SM, Sommer IE. Increased risk of psychosis in patients with hearing impairment: Review and meta-analyses. Neurosci Biobehav Rev. 2016;62:1-20.

12. Varese F, Smeets F, Drukker M, et al. Childhood adversities increase the risk of psychosis: a metaanalysis of patient-control, prospective- and crosssectional cohort studies. Schizophrenia bulletin. 2012;38(4):661-671.

13. Kraan T, Velthorst E, Koenders L, et al. Cannabis use and transition to psychosis in individuals at ultra-high risk: review and meta-analysis. Psychol Med. 2016;46(4):673-681.

14. Davies G, Welham J, Chant D, Torrey EF, McGrath J. A systematic review and meta-analysis of Northern Hemisphere season of birth studies in schizophrenia. Schizophrenia bulletin. 2003;29(3):587-593.

15. Padmanabhan JL, Shah JL, Tandon N, Keshavan MS. The "polyenviromic risk score": aggregating environmental risk factors predicts conversion to psychosis in familial high-risk subjects. Schizophr Res 2017;181:17-22.

16. Dong M, Anda RF, Felitti VJ, et al. The interrelatedness of multiple forms of childhood abuse, neglect, and household dysfunction. Child Abuse Negl. 2004;28(7):771-784.

17. Finkelhor D, Ormrod RK, Turner HA. Polyvictimization: a neglected component in child victimization. Child Abuse Negl. 2007;31(1):7-26

18. Green JG, McLaughlin KA, Berglund PA, et al. Childhood adversities and adult psychiatric disorders in the national comorbidity survey replication I: associations with first onset of DSMIV disorders. Arch Gen Psychiatry. 2010;67(2):113-123.

19. Curtis D. Polygenic risk score for schizophrenia is more strongly associated with ancestry than with schizophrenia. Psychiatr Genet. 2018;28(5):85-89.

20. Christodoulou E, Ma J, Collins GS, Steyerberg EW, Verbakel JY, van Calster B. A systematic review shows no performance benefit of machine learning over logistic regression for clinical prediction models. J Clin Epidemiol. 2019;110:1222.

21. Hand DJ. Classifier technology and the illusion of progress. Statistical Science. 2006;21:1-14

22. Pries LK, Guloksuz S, Ten Have M, et al. Evidence That Environmental and Familial Risks for Psychosis Additively Impact a Multidimensional Subthreshold Psychosis Syndrome. Schizophrenia bulletin. 2018;44(4):710-719.

23. Guloksuz S, van Nierop M, Lieb R, van Winkel R, Wittchen HU, van Os J. Evidence that the presence 
of psychosis in non-psychotic disorder is environment-dependent and mediated by severity of non-psychotic psychopathology. Psychol Med. 2015;45(11):2389-2401.

24. Kirkpatrick B, Tek C, Allardyce J, Morrison G, McCreadie RG. Summer birth and deficit schizophrenia in Dumfries and Galloway, southwestern Scotland. Am J Psychiat. 2002;159(8):1382-1387.

25. Messias E, Kirkpatrick B, Bromet E, et al. Summer birth and deficit schizophrenia: a pooled analysis from 6 countries. Arch Gen Psychiatry. 2004;61(10):985-989.

26. Pries L, Klingenberg B, Menne-Lothmann C, et al. Polygenic Liability for Schizophrenia and Childhood Adversity Influences Daily-Life Emotion Dysregulation and Psychosis Proneness. Acta Psychiatr Scand. 2020;141(5):465-475

27. Arango C, Diaz-Caneja CM, McGorry PD, et al. Preventive strategies for mental health. Lancet Psychiat. 2018;5(7):591-604. 


\title{
Supplementary material
}

\begin{abstract}
Method Summary
Subjects

Data of 1260 patients and 1523 controls from Workpackage 6 of the European Network of National Networks studying GeneEnvironment Interactions in Schizophrenia (EUGEI) ${ }^{17}$ was used as the training dataset and data of 1135 patients, 584 controls and 1045 siblings from the Genetic Risk and Outcome of Psychosis (GROUP) study ${ }^{1}$ were used as the validation dataset. The sample of the EUGEI Workpackage 6 study was collected in Spain, Turkey, and Serbia. The sample of the GROUP study was collected in the Netherlands. Patients were diagnosed with schizophrenia spectrum disorders according to the DSM-IV-TR (average duration of illness since age of first contact with mental health services $=9.9$ years). Participants with complete information on environmental exposures were included in the analyses (Supplementary table S1 shows exposure distributions in the training sample with and without individuals with missing values). The training $(\mathrm{N}=1988)$ and validation $(\mathrm{N}=786)$ dataset includes healthy comparison participants and patients with a diagnosis of schizophrenia spectrum disorders with complete data on environmental factors and demographics. The validation data additionally included healthy siblings $(\mathrm{N}=542)$.
\end{abstract}

\section{Environmental exposures}

Within the limits of data availability, we sought to examine all the environmental exposures that have previously been associated with psychosis spectrum disorders. The environmental exposures were defined in accordance with our previous study investigating gene-environment interactions in these datasets ${ }^{2}$.

Childhood adversity was assessed using the Childhood Trauma Questionnaire Short Form (CTQ) ${ }^{3}$. This consists of 28 items, rated on a 5-point Likert scale, measuring five domains of maltreatment (emotional and physical neglect; emotional, physical and sexual abuse). The psychometric characteristics of the translated versions (Spanish, Turkish, Dutch, and Serbian) of the CTQ have been comprehensively studied ${ }^{4-7}$. To dichotomize each childhood adversity domain $(0=$ "absent" and $1=$ "present"), consistent with previous work in the $\mathrm{EUGEI}^{8}$, we used the following cut-off scores for each domain: $\geq 9$ for emotional abuse; $\geq 8$ for physical abuse; $\geq 6$ for sexual abuse; $\geq 10$ for emotional neglect; and $\geq 8$ for physical neglect.

Cannabis use was assessed by a modified version of the Cannabis Experiences Questionnaire? in the EUGEI ( 0 = "none"; 1 = "only once or twice"; 2 = "a few times a year"; 3 = "a few times a month"; 4 = "once or more a week"; 5 = "everyday"), and by the L section of the Composite International Diagnostic Interview (CIDI) ${ }^{10}$ in the GROUP ( 0 = "none"; 1 = "less than weekly"; 2 = "weekly"; 3 = "daily"). Consistent with previous work ${ }^{11-13}$, a binary regular cannabis use variable was constructed by using the cut-off value of once or more per week during the lifetime period of most frequent use.

In accordance with previous studies investigating the association between season of birth and schizophrenia in the Northern hemisphere $\operatorname{sites}^{14}$, the high-risk birth period was defined based on the winter solstice (December-March), and a binary winter-birth exposure was constructed.

Hearing impairment was defined based on self-reported hearing impairment in the last 12 months $(0=$ "absent" and $1=$ "present").

The history of bullying by peers (emotional, psychological or physical violence) before 17 years of age was assessed using the short version of the Retrospective Bullying Questionnaire $(\mathrm{RBQ})^{15,16}$, which measures the severity of the bullying experience: $0=$ "none"; 1 = "some (no physical injuries)"; 2 = "moderate (minor injuries or transient emotional reactions)"; 3 = "marked (severe and frequent physical or psychological harm)". Exposure to childhood bullying was dichotomized using $\geq 1$ as the cut-off point ( $0=$ "absent" and $\geq 1$ = "present").

\section{Statistical analysis}

Predictive modeling in the training dataset

To impose a balance between patients and controls in the training sample, we selected random samples of patients matching the number of controls (with each participant having the same probability to be selected). To avoid that the sampling process increases the variance of the classification models, the process was repeated for 100 times and the average coefficients were selected. We used MATLAB $^{17}$ to apply logistic regression (LR), Gaussian Naïve Bayes (GNB), and penalized logistic regression models (least absolute shrinkage and selection operator [LASSO] and Ridge) to the balanced case-control training dataset. Ridge and the LASSO classifiers are designed to handle collinearity between variables, while the GNB classifier assumes independence between them. For the 
penalized classification models, an internal 5 -fold cross-validation was used for confirmation and to tune the regularization parameter.

\section{Estimating the predictive power of exposome scores in the validation dataset}

In the validation dataset, ES (the weighted sum of exposures) were calculated based on coefficients from each model. Further, we calculated an environmental sum score of exposure by aggregating environmental factors: participants had scores ranging from 0-9. Guided by the previous study, we estimated an aggregated score based on meta-analyses ${ }^{18}$ including cannabis use ${ }^{19}$, winter birth ${ }^{14}$, hearing impairment ${ }^{20}$, sexual abuse ${ }^{21}$, physical abuse ${ }^{21}$, emotional abuse ${ }^{21}$, neglect ${ }^{21}$ (scored as present if physical neglect or emotional neglect were present), and bullying ${ }^{21}$. Following this, we used the the area under the receiver operating characteristic curve (ROC), accuracy, sensitivity and specificity to assess the predictive ability of ES on case-control status. We also applied logistic regression in STATA version $15.0^{22}$ to analyze ES in patients, controls, and siblings. Nagelkerke's $R^{2}$ was calculated to estimate explained variance. Eventually, we categorized $\mathrm{ES}_{\mathrm{LR}}$ by its quintiles based on the control distribution in the validation dataset and conducted logistic regression with categorical $\mathrm{ES}_{\mathrm{LR}}$ as independent variable. Furthermore, we dichotomized the $\mathrm{ES}_{\mathrm{LR}}$ and compared the bottom $70 \%$ vs the top $30 \%$, the bottom $80 \%$ vs the top $20 \%$, and the bottom $90 \%$ vs the top $10 \%$ of the control distribution.

Supplementary table S1 Distribution of environmental exposures in the training sample

\begin{tabular}{lcccccc}
\hline & \multicolumn{3}{c}{ Without missing values $^{\mathrm{a}}$} & \multicolumn{3}{c}{ With missing values $^{\mathrm{b}}$} \\
\hline & Total & Controls & Patients & Total & Controls & Patients \\
& $=1988$ & $=1241$ & $=747$ & $=2783$ & $=1523$ & $=1260$ \\
Age mean & 33.85 & 33.86 & 33.82 & 33.79 & 33.89 & 33.67 \\
$(\mathrm{SD})$ & $(9.96)$ & $(10.67)$ & $(8.66)$ & $(9.65)$ & $(10.43)$ & $(8.61)$ \\
Females & $43.91 \%$ & $51.25 \%$ & $31.73 \%$ & $42.11 \%$ & $49.38 \%$ & $33.33 \%$ \\
Cannabis use & $11.67 \%$ & $6.20 \%$ & $20.75 \%$ & $13.76 \%$ & $8.12 \%$ & $21.35 \%$ \\
Winter birth & $38.83 \%$ & $38.28 \%$ & $39.76 \%$ & $39.79 \%$ & $39.14 \%$ & $40.61 \%$ \\
Hearing & & & & & & $3.07 \%$ \\
impairment & $6.19 \%$ & $3.14 \%$ & $11.24 \%$ & $5.93 \%$ & & $9.43 \%$ \\
Emotional abuse & $21.28 \%$ & $12.17 \%$ & $36.41 \%$ & $23.40 \%$ & $13.24 \%$ & $38.36 \%$ \\
Physical abuse & $10.87 \%$ & $7.17 \%$ & $17.00 \%$ & $11.48 \%$ & $7.31 \%$ & $17.60 \%$ \\
Sexual abuse & $17.05 \%$ & $9.19 \%$ & $30.12 \%$ & $18.08 \%$ & $9.52 \%$ & $30.52 \%$ \\
Emotional neglect & $56.19 \%$ & $49.80 \%$ & $66.80 \%$ & $55.77 \%$ & $47.90 \%$ & $67.33 \%$ \\
Physical neglect & $38.93 \%$ & $33.60 \%$ & $47.79 \%$ & $38.47 \%$ & $31.67 \%$ & $48.42 \%$ \\
Bullying & $17.91 \%$ & $8.86 \%$ & $32.93 \%$ & $19.56 \%$ & $9.98 \%$ & $31.08 \%$ \\
\hline
\end{tabular}

The table shows the prevalence of exposures (present) within the total sample, controls and patients, ${ }^{2}$ Includes only participants

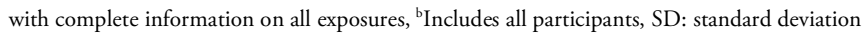

Supplementary table S2 Estimated coefficients from each model in the training sample

\begin{tabular}{|c|c|c|c|c|c|}
\hline Exposures & Meta-analyses $^{a}$ & LR & GNB & Ridge & LASSO \\
\hline Cannabis use & 0.56 & 1.31 & 1.33 & 1.46 & 1.53 \\
\hline Winter-birth & 0.07 & 0.03 & 0.01 & 0.06 & 0.06 \\
\hline Hearing impairment & 1.15 & 1.18 & 1.19 & 1.10 & 1.16 \\
\hline Emotional abuse & 1.22 & 0.78 & 1.42 & 0.87 & 0.91 \\
\hline Physical abuse & 1.08 & -0.39 & 0.94 & -0.22 & -0.27 \\
\hline Sexual abuse & 0.87 & 0.86 & 1.45 & 0.66 & 0.68 \\
\hline Emotional neglect & \multirow{2}{*}{1.06} & 0.44 & 0.78 & 0.43 & 0.45 \\
\hline Physical neglect & & 0.25 & 0.66 & 0.11 & 0.10 \\
\hline Bullying & 0.87 & 1.35 & 1.57 & 1.30 & 1.35 \\
\hline
\end{tabular}

${ }^{2}$ Guided by the previous approach ${ }^{18}$, the exposome score was calculated using log odds ratios from meta-analyses ${ }^{14,19-21}=$

$\left(0.56^{*}\right.$ cannabis use $+0.068^{*}$ winter birth $+1.15^{*}$ hearing impairment $+1.22^{*}$ emotional abuse $+1.08^{*}$ physical abuse +

$0.87^{*}$ sexual abuse $+1.06^{*}$ neglect $+0.87^{*}$ bullying $) / 8$, neglect was scored as present if physical neglect or emotional neglect was present, GNB: Gaussian Naïve Bayes, LR: logistic regression 
Supplementary table S3 Predictive ability of exposome scores in the validation dataset

\begin{tabular}{lcccc}
\hline & ROC & Accuracy & Sensitivity & Specificity \\
\hline ES $_{\text {Meta-analyses }}$ & 0.69 & 0.62 & 0.56 & 0.72 \\
Sum score & 0.71 & 0.67 & 0.74 & 0.58 \\
ES $_{\text {LR }}$ & 0.73 & 0.68 & 0.86 & 0.42 \\
ES $_{\text {GNB }}$ & 0.71 & 0.68 & 0.72 & 0.61 \\
ES & 0.74 & 0.68 & 0.86 & 0.42 \\
ES & 0.74 & 0.68 & 0.86 & 0.42 \\
\hline
\end{tabular}

ES: exposome score, GNB: Gaussian Naïve Bayes, LR: logistic regression, ROC: area under the receiver operating characteristic curve

Supplementary table S4 Exposome scores in patients, siblings and controls in the validation dataset

\begin{tabular}{|c|c|c|c|c|c|c|c|c|c|c|}
\hline & \multicolumn{4}{|c|}{ Patients vs Controls } & \multicolumn{3}{|c|}{ Patients vs Siblings } & \multicolumn{3}{|c|}{ Sibling vs Controls } \\
\hline & OR & $95 \% \mathrm{CI}$ & $\begin{array}{c}P_{-} \\
\text {value }\end{array}$ & $R^{2}$ & OR & $95 \% \mathrm{CI}$ & $\begin{array}{c}P_{-} \\
\text {value }\end{array}$ & OR & $95 \% \mathrm{CI}$ & $\begin{array}{c}P- \\
\text { value }\end{array}$ \\
\hline \multirow[t]{2}{*}{$\begin{array}{l}\mathrm{ES}_{\text {Meta- }} \\
\text { analyes }\end{array}$} & $40.65^{\mathrm{a}}$ & $\begin{array}{c}17.06 \text { to } \\
96.84\end{array}$ & $<0.001$ & 0.13 & 14.33 & $\begin{array}{l}7.12 \text { to } \\
28.82\end{array}$ & $<0.001$ & 2.91 & $\begin{array}{c}1.29 \text { to } \\
6.56\end{array}$ & 0.010 \\
\hline & $1.59^{\mathrm{b}}$ & $\begin{array}{c}1.43 \text { to } \\
1.77\end{array}$ & $<0.001$ & 0.13 & 1.39 & $\begin{array}{c}1.28 \text { to } \\
1.52\end{array}$ & $<0.001$ & 1.14 & $\begin{array}{c}1.03 \text { to } \\
1.27\end{array}$ & 0.010 \\
\hline $\begin{array}{l}\text { Sum } \\
\text { score }\end{array}$ & 1.59 & $\begin{array}{l}1.44 \text { to } \\
1.75\end{array}$ & $<0.001$ & 0.17 & 1.36 & $\begin{array}{c}1.27 \text { to } \\
1.47\end{array}$ & $<0.001$ & 1.15 & $\begin{array}{c}1.06 \text { to } \\
1.26\end{array}$ & 0.002 \\
\hline $\mathrm{ES}_{\mathrm{LR}}$ & 1.94 & $\begin{array}{c}1.71 \text { to } \\
2.20\end{array}$ & $<0.001$ & 0.21 & 1.58 & $\begin{array}{c}1.43 \text { to } \\
1.74\end{array}$ & $<0.001$ & 1.21 & $\begin{array}{c}1.08 \text { to } \\
1.36\end{array}$ & 0.001 \\
\hline $\mathrm{ES}_{\mathrm{GNB}}$ & 1.49 & $\begin{array}{c}1.37 \text { to } \\
1.63\end{array}$ & $<0.001$ & 0.18 & 1.33 & $\begin{array}{c}1.24 \text { to } \\
1.42\end{array}$ & $<0.001$ & 1.12 & $\begin{array}{c}1.04 \text { to } \\
1.21\end{array}$ & 0.003 \\
\hline ES RIDGE & 2.00 & $\begin{array}{l}1.75 \text { to } \\
2.27\end{array}$ & $<0.001$ & 0.22 & 1.61 & $\begin{array}{c}1.46 \text { to } \\
1.78\end{array}$ & $<0.001$ & 1.22 & $\begin{array}{c}1.09 \text { to } \\
1.37\end{array}$ & $<0.001$ \\
\hline $\mathrm{ES}_{\text {LASSO }}$ & 1.95 & $\begin{array}{c}1.72 \text { to } \\
2.21\end{array}$ & $<0.001$ & 0.22 & 1.58 & $\begin{array}{c}1.44 \text { to } \\
1.74\end{array}$ & $<0.001$ & 1.22 & $\begin{array}{c}1.09 \text { to } \\
1.36\end{array}$ & $<0.001$ \\
\hline
\end{tabular}

${ }^{a}$ The higher ORs of the ES $S_{\text {Meta-analyes }}$ are due to the scaling of the ES resulting from dividing the sum score by 8, ${ }^{\text {b ORs of the ES }}$ Mera analyses when the sum score was not divided by 8 , CI: confidence interval, ES: exposome score, GNB: Gaussian Naïve Bayes, LR: logistic regression, OR: Odds ratio, $R^{2}$ : Nagelkerke's pseudo $R^{2}$

Supplementary table S5 Testing binarized exposome score in patients and controls in the validation dataset

\begin{tabular}{lllll}
\hline High ES definition & Reference group & OR $^{\mathrm{a}}$ & $95 \% \mathrm{CI}$ & $P$-value \\
\hline Top $30 \%$ of distribution & Remaining $70 \%$ & 3.72 & 2.68 to 5.17 & $<0.001$ \\
Top 20\% of distribution & Remaining $80 \%$ & 3.74 & 2.63 to 5.32 & $<0.001$ \\
Top $10 \%$ of distribution & Remaining $90 \%$ & 4.77 & 3.06 to 7.44 & $<0.001$
\end{tabular}

The binary categories of the ES $S_{\mathrm{LR}}$ were used in the logistic regression analysis. ${ }^{2}$ Adjusted for sex and age, CI: confidence interval, OR: Odds ratio 


\section{References}

1. Korver N, Quee PJ, Boos HB, Simons CJ, de Haan $\mathrm{L}$, investigators $\mathrm{G}$. Genetic Risk and Outcome of Psychosis (GROUP), a multi-site longitudinal cohort study focused on gene-environment interaction: objectives, sample characteristics, recruitment and assessment methods. Int J Methods Psychiatr Res. 2012;21(3):205-221.

2. Guloksuz S, Pries LK, Delespaul P, et al. Examining the independent and joint effects of molecular genetic liability and environmental exposures in schizophrenia: results from the EUGEI study. World Psychiatry. 2019;18(2):173-182.

3. Bernstein DP, Stein JA, Newcomb MD, et al. Development and validation of a brief screening version of the Childhood Trauma Questionnaire. Child Abuse Negl. 2003;27(2):169-190.

4. Sar V, Akyuz G, Kundakci T, Kiziltan E, Dogan O. Childhood trauma, dissociation, and psychiatric comorbidity in patients with conversion disorder. The American journal of psychiatry. 2004;161(12):2271-2276.

5. Hernandez A, Gallardo-Pujol D, Pereda N, et al. Initial validation of the Spanish childhood trauma questionnaire-short form: factor structure, reliability and association with parenting. $J$ Interpers Violence. 2013;28(7):1498-1518.

6. Thombs BD, Bernstein DP, Lobbestael J, Arntz A. A validation study of the Dutch Childhood Trauma Questionnaire-Short Form: factor structure, reliability, and known-groups validity. Child Abuse Negl. 2009;33(8):518-523.

7. Mitkovic Voncina M, Lecic Tosevski D, Pejovic Milovancevic M, Popovic Deusic SJABS. Linking child maltreatment history with child abuse potential: relative roles of maltreatment types. 2014;66(4):1681-1687.

8. Kraan TC, Velthorst E, Themmen M, et al. Child maltreatment and clinical outcome in individuals at ultra-high risk for psychosis in the EU-GEI high risk study. Schizophr Bull. 2017;44(3):584-592.

9. Barkus EJ, Stirling J, Hopkins RS, Lewis S. Cannabis-induced psychosis-like experiences are associated with high schizotypy. Psychopathology. 2006;39(4):175-178.

10. Robins LN, Wing J, Wittchen HU, et al. The Composite International Diagnostic Interview - an Epidemiologic Instrument Suitable for Use in Conjunction with Different Diagnostic Systems and in Different Cultures. Arch Gen Psychiatry. 1988;45(12):1069-1077.
11. van Winkel R, van Beveren NJ, Simons C, GROUP Investigators. AKT1 moderation of cannabis-induced cognitive alterations in psychotic disorder. Neuropsychopharmacology. 2011;36(12):2529-2537.

12. Pries LK, Guloksuz S, Ten Have M, et al. Evidence That Environmental and Familial Risks for Psychosis Additively Impact a Multidimensional Subthreshold Psychosis Syndrome. Schizophr Bull. 2018;44(4):710-719.

13. Radhakrishnan R, Guloksuz S, Ten Have M, et al. Interaction between environmental and familial affective risk impacts psychosis admixture in states of affective dysregulation. Psychol Med. 2018:1-11.

14. Davies G, Welham J, Chant D, Torrey EF, McGrath J. A systematic review and meta-analysis of Northern Hemisphere season of birth studies in schizophrenia. Schizophr Bull. 2003;29(3):587593.

15. Schafer M, Korn S, Smith PK, et al. Lonely in the crowd: Recollections of bullying. British Journal of Developmental Psychology. 2004;22(3):379-394.

16. Hunter SC, Mora-Merchan J, Ortega R. The LongTerm Effects of Coping Strategy Use in Victims of Bullying. The Spanish journal of psychology. 2014;7(01):3-12.

17. MathWorks I. MATLAB : the language of technical computing : computation, visualization, programming : installation guide for UNIX version 5. Natwick: Math Works Inc; 1996.

18. Padmanabhan JL, Shah JL, Tandon N, Keshavan MS. The "polyenviromic risk score": aggregating environmental risk factors predicts conversion to psychosis in familial high-risk subjects. Schizophr Res 2017;181:17-22.

19. Kraan T, Velthorst E, Koenders L, et al. Cannabis use and transition to psychosis in individuals at ultra-high risk: review and meta-analysis. Psychol Med. 2016;46(4):673-681.

20. Linszen MM, Brouwer RM, Heringa SM, Sommer IE. Increased risk of psychosis in patients with hearing impairment: Review and meta-analyses. Neurosci Biobehav Rev. 2016;62:1-20.

21. Varese F, Smeets F, Drukker M, et al. Childhood adversities increase the risk of psychosis: a metaanalysis of patient-control, prospective- and crosssectional cohort studies. Schizophrenia bulletin. 2012;38(4):661-671.

22. STATA [computer program]. Version 15. TX: StataCorp LLC; 2017 



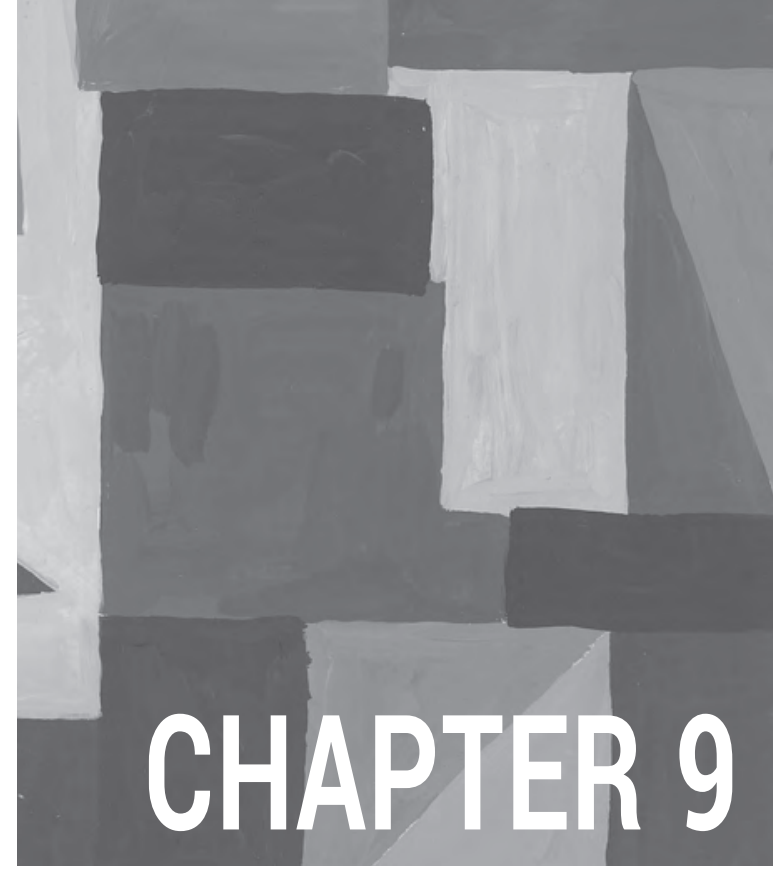

\section{ASSOCIATION OF RECENT STRESSFUL LIFE EVENTS WITH MENTAL AND PHYSICAL HEALTH IN THE CONTEXT OF GENOMIC AND EXPOSOMIC LIABILITY FOR SCHIZOPHRENIA}

Lotta-Katrin Pries, Jim van Os, Margreet ten Have, Ron de Graaf, Saskia van Dorsselaer, Maarten Bak, Bochao D. Lin, Kristel R. van Eijk, Gunter Kenis, Alexander Richards, Michael C. O'Donovan, Jurjen J. Luykx, Bart P. F. Rutten, Sinan Guloksuz

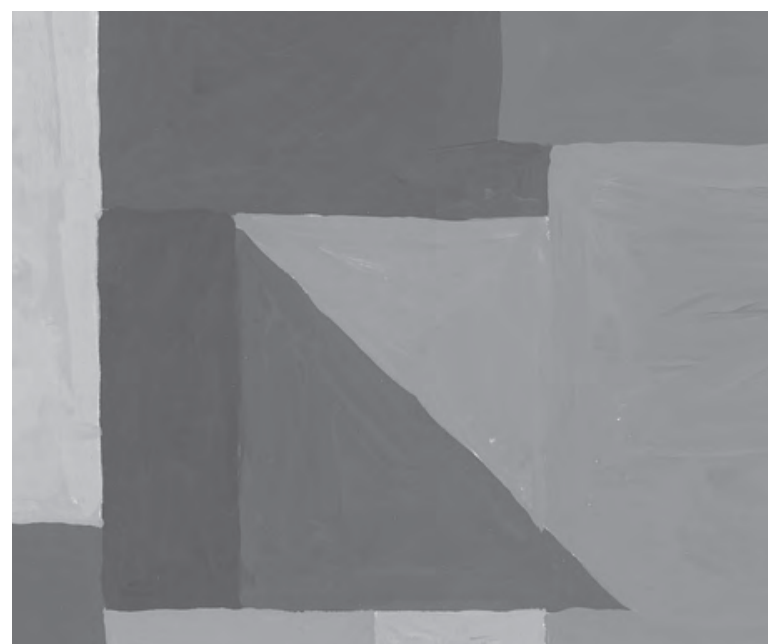




\section{Key Points}

\section{Question}

Do adulthood stressful life events interact with polygenic risk and exposome scores for schizophrenia in models of mental and physical health?

\section{Findings}

This longitudinal study provides novel evidence for the contributions of genetic and environmental liability for schizophrenia to mental health in the general population. Both genetic and environmental liability were associated with poor outcomes, particularly mental health. The association between stressful life-events and health outcomes was moderated by exposome score but not polygenic risk for schizophrenia.

\section{Meaning}

Our findings showing an interaction between adulthood stressful life events and lifetime exposomic liability lend further support to the diathesis-stress model. 


\section{Abstract}

Importance: Both adulthood stressful life events (SLEs) and liability for schizophrenia have been associated with poor mental and physical health in the general population, but their interaction remains to be elucidated to improve population-based health outcomes.

Objective: To test whether recent SLEs interact with genetic and environmental liability for schizophrenia in models of mental and physical health.

Design: NEMESIS-2 is a population-based prospective cohort study designed to investigate the prevalence, incidence, course, and consequences of mental disorders.

Settings: Dutch general population.

Participants: Participants were enrolled in 2007-2009 at baseline and followed up with three assessments over nine years.

Exposures: Recent SLEs assessed at each wave and aggregate scores of genetic and environmental liability for schizophrenia: polygenic risk score for schizophrenia (PRS-SCZ) trained using the PGC 2 results and exposome score for schizophrenia (ES-SCZ) trained using an independent data set.

Main Outcomes and Measures: Independent and interacting associations of SLEs with ESSCZ and PRS-SCZ on mental and physical health assessed at each wave.

Results: Of the 6,646 participants included at baseline, the mean (SD) age was 44 (13) years and $55 \%$ were female. SLEs was associated with poorer physical health $(B=-3.22$ [95\% CI: $-3.66,-2.79])$ and mental health $(B=-3.68$ [95\% CI: $-4.05,-3.32])$. Genetic and environmental liability for schizophrenia were associated with poorer mental health (ESSCZ: $B=-3.07$ [95\% CI: -3.35, -2.79]; PRS-SCZ: $B=-0.93$ [95\% CI: $-1.31,-0.54]$ ). Environmental liability was also associated with poorer physical health $(B=-3.19$ [95\% CI: $-3.56,-2.82])$. The interaction model showed that ES-SCZ moderated the association of SLEs with mental $(B=-1.08$ [95\% CI: $-1.47,-0.69])$ and physical health $[B=-0.64(95 \%$ CI: $-1.11,-0.17)]$, while PRS-SCZ did not. Several sensitivity analyses confirmed these results.

Conclusions and Relevance: Schizophrenia liability contributes to broad mental health outcomes at the population level. Consistent with the diathesis-stress model, exposure to SLEs, particularly in individuals with high environmental liability for schizophrenia, was associated with poorer health. Our findings underline the importance of modifiable environmental factors over the life span for the population-based mental health outcomes.

Keywords: Exposome, stressful life events, polygenic risk score, environment, psychosis, mental health, gene-environment interaction 


\section{Introduction}

Accumulating evidence indicates that adulthood stressful life events (SLEs) contribute to the onset and outcome of a broad range of mental disorders ${ }^{1-4}$. Further, SLEs are key determinants of poor mental and physical health in the general population ${ }^{2,35-11}$. Although SLEs are highly relevant for mental and physical wellbeing, they are seldom screened in routine clinical practice. A recent viewpoint has therefore called for integration of the SLE dimension to maximize the clinical utility of electronic health records ${ }^{12}$.

SLEs are ubiquitous, with $30-40 \%$ of the general population experiencing at least one major SLE (e.g., death of a relative) each year ${ }^{6,13}$. However, the majority of people do not develop mental disorders after experiencing a SLE. According to the diathesis-stress model ${ }^{14}$, one possible explanation is that early life events increase the vulnerability to SLEs later in life. Converging evidence for the diathesis-stress model stems from epidemiological studies focusing on the influence of the environment on psychopathology over the life-span. Early adverse events, such as childhood adversity, moderate the association between adulthood SLEs and a broad range of multidimensional psychopathology outcomes across the severity spectrum from depression to psychosis ${ }^{6,15-21}$. Further, the influences of exposures on psychopathology appear to accumulate in a dose-response fashion: the more severe the exposure, the more severe the outcome $\mathrm{e}^{22-27}$.

Similar to the antecedent environmental liability to SLEs, genetic background may emerge as a predisposing factor for the detrimental impact of SLEs on health. The liability-threshold model ${ }^{28}$ posits that phenotypic outcomes can be quantified by the combination of cumulative genetic and environmental liability, where individuals passing the critical threshold develop expression of the disease phenotype. Recent insight from genome-wide association studies (GWAS), enabling polygenic risk estimation of phenotypes (polygenic risk score [PRS]: a weighted sum of trait-associated alleles), has galvanized research into polygenic theory of psychopathology and provided a single metric of molecular genetic liability for geneenvironment interaction research. We have shown that PRS for schizophrenia (PRS-SCZ) interacts with childhood adversities and cannabis use, increasing the likelihood of schizophrenia ${ }^{29}$. A recent study pairing PRS-SCZ with an aggregate environmental score provided further evidence for gene-environment interaction in a first episode psychosis sample ${ }^{30,31}$.

The recent series of studies testing the diathesis-stress model of depression in independent samples show evidence for a positive interaction between PRS for major depressive disorder (MDD) and SLEs increasing depression risk ${ }^{32-34}$. The interaction between PRS-MDD and 
SLEs appears to be pleiotropic, extending to not only mental health phenotypes (e.g., schizotypal personality) but also physical health phenotypes, such as cardiovascular disease and chronic obstructive pulmonary disease ${ }^{34}$. The shared stress-vulnerability across disease phenotypes is an anticipated outcome given the pleiotropic associations of PRS and SLEs.

PRS-SCZ clearly outperforms the rest of the PRSs for mental disorder phenotypes that have been estimated so far, and appears to be the forerunner for developing PRS-based clinical applications ${ }^{35,36}$. Genetic liability for schizophrenia shows significant overlap with genetic liability across mental health phenotypes ${ }^{37}$. PRS-SCZ is associated with a variety of disorders ${ }^{35,38,39}$, quality of life $\mathrm{e}^{40}$, and subclinical multidimensional phenotypes ${ }^{39,41-43}$. Further, the investigation of the electronic health records from the United States revealed that PRSSCZ was associated with not only a diagnosis of schizophrenia but also diagnoses of related psychiatric and other medical conditions ${ }^{35}$.

Taken together, these findings indicate that an impact of SLEs is to a degree contingent on an individual's environmental and genetic liability. The findings of our recent study provide further support to the pleiotropic impact of stress-vulnerability: PRS-SCZ interacted with stressful life events moderating the daily variations of negative affect, positive affect, and psychosis expression in the general population ${ }^{44}$. To date, the diathesis-stress theory for SLEs by using PRS for genetic liability estimation has only been tested cross-sectionally, while environmental liability remains to be estimated as a single metric (analogous to PRS) to embrace the dense network of exposures in general population cohorts.

Here, we aimed to embrace the complexity of many-to-many non-specific relationships in psychiatry ${ }^{45}$ by investigating to what degree all-encompassing health outcomes at the population level were influenced as a function of the interaction between SLEs ("precipitating events") and liability for arguably the most severe and chronic form of mental disorders ("predisposition"), schizophrenia, considering the fact that the genetic and environmental liability for schizophrenia, as the selection on the extreme end of the mental disorder severity spectrum, should plausibly have a greater impact on mental health at the population level. By making use of a population-based prospective cohort that includes detailed information on early life environmental exposures, GWAS data, and repeated assessments of adulthood SLEs and health outcomes, we investigated whether the association of SLEs with mental and physical health was moderated by PRS-SCZ and exposome score for schizophrenia (ESSCZ), a composite score of environmental liability for schizophrenia ${ }^{46}$. 


\section{Method}

\section{Study Population}

The Netherlands Mental Health Survey and Incidence Study-2 (NEMESIS-2) was conducted to study the prevalence, incidence, course, and consequences of mental disorders in the Dutch general population. The study was approved by the Medical Ethics Review Committee for Institutions on Mental Health Care and written informed consent was collected from participants at each wave. To ensure representativeness of the sample in terms of age (between the ages of 18 and 65 at baseline), region, and population density, a multistage random sampling procedure was applied. Dutch illiteracy was an exclusion criterion. Non-clinician, trained interviewers applied the Composite International Diagnostic Interview (CIDI) version 3.0 $0^{47,48}$ and additional questionnaires during home visits. Details of NEMESIS-2 were provided elsewhere ${ }^{49,50}$. From 2007 to 2009 , the first wave (T0) enrolled 6,646 participants (response rate 65.1\%; average interview duration: 95 minutes), who were followed up at 3 visits within 9 years: successive response rates at year 3 (T1), year 6 (T2), and year 9 (T3) were $80.4 \%(n=5,303$; excluding those who deceased; interview duration: 84 minutes), $87.8 \%$ ( $n=4,618$; interview duration: 83 minutes), and $86.8 \%$ ( $n=4,007$; interview duration: 102 minutes), respectively. Data from all four waves were utilized. Rates at baseline reflect lifetime occurrence; rates at T1 to T3 reflect 3-year interval (baseline-T1, T1-T2, and T2-T3) occurrence. Attrition between T0 and T3 was not significantly associated with any of the individual 12-month mental disorders at T0 after controlling for sociodemographic characteristics ${ }^{51}$. Genetic data were available in a subsample of 3,104 individuals.

\section{Measurements}

\section{Exposome score for schizophrenia}

Guided by the approach validated in a large international study ${ }^{46}$, the continuous ES-SCZ was calculated by summing log odds weighted environmental exposures (cannabis use, winter birth, hearing impairment, and childhood adversities [emotional neglect, psychological abuse, physical abuse, sexual abuse, and peer victimization]) at baseline (eMethods).

\section{Adulthood stressful life events}

Based on the "Brugha Life events section" 52 , participants were asked whether they experienced one of 9 life events within the last 12 month (T0) or since the last interview (T1 to T3) (eTable 2). Consistent with previous work from this cohort ${ }^{53,54}$, we used the single binary SLEs variable (present: $\geq 1$ life events and absent: 0 life events). 


\section{Mental health and physical health}

The Short-Form-36 Health Survey (SF-36) ${ }^{55}$ consists of nine subscales, each scale ranging from poor (0) to good (100) functioning. Based on the SF-36 manual ${ }^{56}$, mental health, role limitations due to emotional problems, social functioning, and vitality scales were averaged into a mental health dimension, while general health perceptions, physical functioning, role limitation due to physical health problems, and bodily pain scales were averaged into the physical health dimension ${ }^{57}$. The SF-36 was assessed at each time-point and refers to the past four weeks.

\section{Polygenic risk score for schizophrenia}

PRS-SCZ was created from best estimate genotypes at six different $P$-thresholds $(0.5,0.1$, $0.05,5 \cdot 10^{-3}, 5 \cdot 10^{-5}, 5 \cdot 10^{-8}$ ) (eMethods). For our primary analyses, we used the $P$-threshold of $<0.05$, as this threshold optimally captures liability to the disorder in the Psychiatric Genomics Consortium analysis ${ }^{58}$ and performed well for the current phenotypes (eTable 3).

\section{Statistical analyses}

All analyses were performed using Stata, version $15^{59} . P<0.05$ (2-tailed) was considered nominally statistically significant. Baseline correlation between PRS-SCZ and SLEs was tested using logistic regression models. Using data from all four assessment points, we fitted multilevel mixed regression models using the MIXED command in Stata to test the association of recent SLEs, ES-SCZ, and PRS-SCZ with mental and physical health outcomes. SLEs and health outcomes were assessed at each time-point and therefore timevarying; the genetic (PRS-SCZ) and environmental liability (ES-SCZ) were antecedents and time-invariant. Likelihood ratio tests comparing models with different covariance structures (independent and autoregressive) indicated that a first order autoregressive (AR1) covariance structure $^{60,61}$ was the most suitable for the current analyses. The continuous independent variables (PRS-SCZ and ES-SCZ) were standardized and centered $(\mathrm{min}=0, \mathrm{SD}=1)$ at baseline. Given the hierarchical structure of the data, multilevel mixed-effect models were applied to cluster the multiple assessments per individuals. All analyses were additionally a priori adjusted for age, sex, and 4-level education (primary school, lower secondary, higher secondary, and higher professional education). Models including PRS-SCZ were conducted in a subsample of 3104 genotyped individuals and additionally adjusted for three principal components. To adequately control for confounding ${ }^{62}$, interaction models with PRS-SCZ included the covariates as well as covariate $\mathrm{x}$ SLEs and covariate $\mathrm{x}$ PRS-SCZ interaction terms. Univariate and multiple logistic regression models were applied to compare the baseline characteristics between individuals who completed all four assessments and those who dropped-out at any time-point. The multivariable model indicated that younger age and lower education at baseline were associated with attrition over the study period (eTable 4). 
We additionally tested the associations of the environmental exposures (ES-SCZ and SLEs) in the genotyped subsample. Further, the analyses were conducted after the imputation of missing observations (Table 1) in environmental exposure assessments and health outcomes. Imputation and subsequent analyses were separately conducted for the total and genotyped subsample. Assuming missing at random, we applied the multiple imputation chained equation model $^{63}$ with 20 imputations which were limited to in-range values (relative efficiency was above 99\%). Subsequent analyses were based on multiply imputed data with estimates being pooled using Rubin's rules ${ }^{64}$.

To test the robustness of our findings, we conducted several sensitivity analyses. We first tested whether the results of recent SLEs converged to previous SLEs (at time point t-1), aiming to assess temporal association and proximity. Further, the analyses were conducted by replacing independent variables one at a time with the following sensitivity measures: PRS-SCZ with a $P$-threshold of $<0.1$ (PRS-SCZ 0.1 ), polygenic risk score for major depressive disorders (PRS-MDD), ES-SCZ without cannabis use history, the sum score of recent SLEs (tSLEs), recent SLEs split into independent (iSLEs) and dependent (dSLEs). Finally, we repeated the primary analyses using the narrow dimensions of mental health (mental health and role limitations due to emotional problems scales) and physical health (physical functioning, role limitation due to physical health problems, and bodily pain) as outcomes ${ }^{56}$ (eMethods).

\section{Results}

Table 1 reports sample characteristics at the different time-points and missing values at baseline. Regression analyses of baseline only data revealed that recent SLEs explained 5\% ( $P$ $<0.001)$ and $3.7 \%(P<0.001)$ of the variance in baseline mental and physical health respectively, while ES-SCZ explained $5.5 \%(P<0.001)$ and $3.2 \%(P<0.001)$ of the variance in baseline mental and physical health, respectively. PRS-SCZ at a $P$-threshold of $<0.05$ explained $0.5 \%$ of the variance in baseline mental health $(P<0.001)$, but PRS-SCZ was not significantly associated with physical health at baseline (eTable 3$)$. 
Table 1 Sample characteristics at the different assessment points and missing values at baseline

\begin{tabular}{|c|c|c|c|c|c|}
\hline Variables & $\begin{array}{c}\text { Baseline } \\
\text { sample } \\
(\mathrm{n}=6646)\end{array}$ & $\begin{array}{c}\mathrm{T} 1 \\
(\mathrm{n}=5303)\end{array}$ & $\begin{array}{c}\text { T2 } \\
(\mathrm{n}=4618)\end{array}$ & $\begin{array}{c}\text { T3 } \\
(\mathrm{n}=4007)\end{array}$ & $\begin{array}{c}\text { Number of missing } \\
\text { observations } \\
\text { at baseline }\end{array}$ \\
\hline $\begin{array}{l}\text { Age, mean } \\
\text { (SD) }\end{array}$ & $\begin{array}{c}44.26 \\
(12.54)\end{array}$ & $\begin{array}{l}47.61 \\
(12.37)\end{array}$ & $\begin{array}{l}50.87 \\
(12.32)\end{array}$ & $\begin{array}{c}54.06 \\
(12.28)\end{array}$ & - \\
\hline Sex (\% female) & 55.25 & 55.10 & 55.39 & 55.53 & - \\
\hline \multicolumn{6}{|l|}{ Education (\%) } \\
\hline Primary school & 5.00 & 4.26 & 4.03 & 3.84 & - \\
\hline Lower secondary & 27.48 & 26.17 & 25.83 & 25.51 & - \\
\hline Higher secondary & 32.28 & 32.59 & 32.03 & 31.79 & - \\
\hline Higher professional & 35.25 & 36.98 & 38.11 & 38.86 & - \\
\hline $\begin{array}{l}\text { Mental health, mean } \\
\text { (SD) }\end{array}$ & $\begin{array}{l}84.85 \\
(13.97)\end{array}$ & $\begin{array}{l}82.57 \\
(14.56)\end{array}$ & $\begin{array}{c}82.88 \\
(14.70)\end{array}$ & $\begin{array}{l}84.69 \\
(14.24)\end{array}$ & 3 \\
\hline $\begin{array}{l}\text { Physical health, mean } \\
\text { (SD) }\end{array}$ & $\begin{array}{l}84.09 \\
(17.67)\end{array}$ & $\begin{array}{c}82.33 \\
(18.70)\end{array}$ & $\begin{array}{l}81.26 \\
(19.24)\end{array}$ & $\begin{array}{c}80.87 \\
(19.42)\end{array}$ & 1 \\
\hline $\begin{array}{l}\text { Stressful life events (\% } \\
\text { present) }\end{array}$ & 50.60 & 73.60 & 76.38 & 76.82 & 140 \\
\hline $\begin{array}{l}\text { Exposome score for } \\
\text { schizophrenia, mean } \\
(\mathrm{SD})^{\mathrm{a}}\end{array}$ & $\begin{array}{c}0.52 \\
(0.83)\end{array}$ & $\begin{array}{c}0.53 \\
(0.83)\end{array}$ & $\begin{array}{c}0.52 \\
(0.83)\end{array}$ & $\begin{array}{c}0.52 \\
(0.82)\end{array}$ & 380 \\
\hline
\end{tabular}

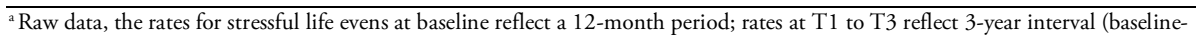
T1, T1-T2, and T2-T3) occurrence; SD: Standard deviation

The longitudinal mixed regression models indicated that recent SLEs were associated with poorer mental health $(B=-3.64$ [95\%CI: $-4.00,-3.28], P<0.001)$ and physical health $(B=$ -3.69 [95\%CI: $-4.12,-3.26$ ] $P<0.001)$. ES-SCZ was likewise associated with poorer mental health $(B=-3.16$ [95\%CI: $-3.44,-2.88], P<0.001)$ and physical health $(B=-3.09$ [95\%CI: $-3.48,-2.71], P<0.001)$. Increased PRS-SCZ was associated with decreased mental health $(B=-0.90$ [95\%CI: $-1.30,-0.51], P<0.001)$, but no significant association was found between PRS-SCZ and physical health $(B=-0.38$ [95\%CI: $-0.92,0.17$ ], $P=0.17)$. The results were similar after adjusting for age, sex, and education (Table 2 ). 
Table 2 Main associations and interactions of recent stressful life events, exposome score for schizophrenia, and polygenic risk score for schizophrenia on health outcomes

\begin{tabular}{|c|c|c|c|c|c|c|}
\hline & \multicolumn{3}{|c|}{ Mental health } & \multicolumn{3}{|c|}{ Physical health } \\
\hline & $B$ & $95 \% \mathrm{CI}$ & $P$-value & $B$ & $95 \% \mathrm{CI}$ & $P$-value \\
\hline \multicolumn{7}{|l|}{ Main associations } \\
\hline SLE & -3.68 & $\begin{array}{c}-4.05 \text { to }- \\
3.32\end{array}$ & $<0.001$ & -3.22 & $\begin{array}{c}-3.66 \text { to }- \\
2.79\end{array}$ & $<0.001$ \\
\hline ES-SCZ & -3.07 & $\begin{array}{c}-3.35 \text { to }- \\
2.79\end{array}$ & $<0.001$ & -3.19 & $\begin{array}{c}-3.56 \text { to }- \\
2.82\end{array}$ & $<0.001$ \\
\hline PRS-SCZ & -0.93 & $\begin{array}{c}-1.31 \text { to }- \\
0.54\end{array}$ & $<0.001$ & -0.49 & -1.01 to 0.03 & 0.06 \\
\hline \multicolumn{7}{|l|}{ Interactions } \\
\hline SLE $x$ ES-SCZ & -1.08 & $\begin{array}{c}-1.47 \text { to }- \\
0.69\end{array}$ & $<0.001$ & -0.64 & $\begin{array}{c}-1.11 \text { to }- \\
0.17\end{array}$ & 0.008 \\
\hline SLE $x$ PRS-SCZ ${ }^{a}$ & -0.25 & -0.74 to 0.24 & 0.33 & -0.17 & -0.76 to 0.42 & 0.58 \\
\hline
\end{tabular}

All analyses were adjusted for sex, age, and education; additionally adjusted for three principal components; $B$ : Regression coefficient from the multilevel mixed models; CI: Confidence Interval; ES-SCZ: Exposome score for schizophrenia; PRS-SCZ:

Polygenic risk score for schizophrenia; SLE: Stressful life events

There was evidence for a significant interaction between recent SLEs and ES-SCZ on mental health $(B=-1.09$ [95\%CI: $-1.48,-0.70], P<0.001)$ and physical health $(B=-0.63$ [95\%CI: $-1.11,-0.16], P=0.009$ ), also after adjusting for age, sex, and education (Table 2 and Figure 1). No gene-environment correlation was detected: no large or significant association was found between PRS-SCZ and recent SLEs (OR=1.03 [95\%CI: 0.96, 1.10], $P=0.45$ ). No evidence was found for an interaction between recent SLEs and PRS-SCZ on mental $(B=-$ 0.25 [95\%CI: $-0.73,0.23$ ], $P=0.31)$ or physical health $(B=-0.14$ [95\%CI: $-0.72,0.45], P$ $=0.65)$, also after adjusting for age, sex, and education. In accordance with the analyses in the total sample, adjusted main effect analyses in the genotyped subsample revealed that recent SLEs were associated with poorer mental health and physical health. Further, ES-SCZ was associated with poorer mental health and physical health in the genotyped subsample (eTable 5). Analyses on imputed results also converged with those in the unimputed dataset (eTable 6). 

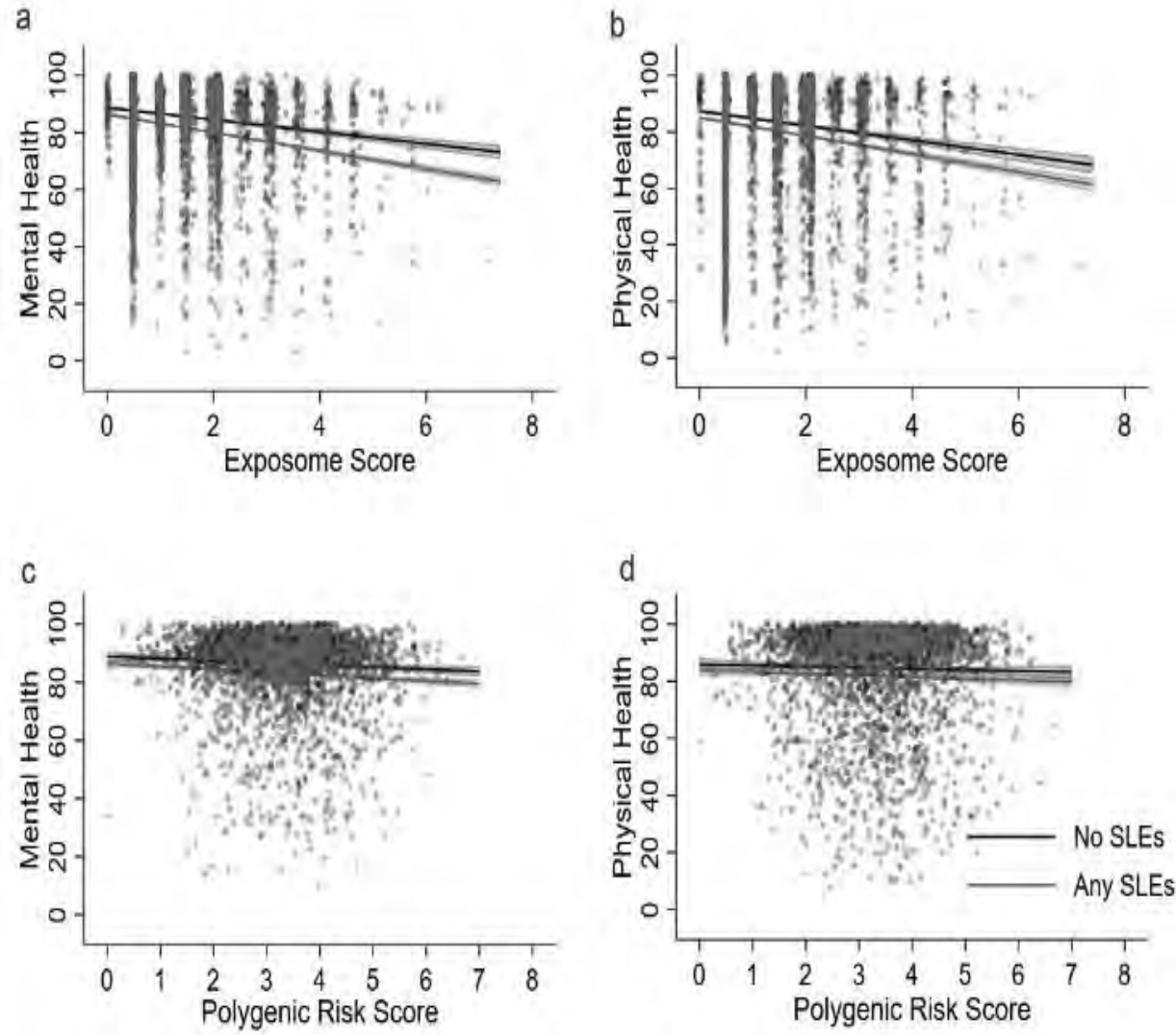

Figure a) and b) show marginal effect plots based on adjusted multilevel mixed regression of the interaction between exposome score for schizophrenia (x-axis) and recent stressful life events (SLEs) on continuous measures of mental health and physical health (y-axis). Raw data associations between exposome score for schizophrenia and baseline health outcome by recent SLEs are visualized as scatter; Figure c) and d) show marginal effect plots based on adjusted multilevel mixed regression of the interaction between polygenic risk score for schizophrenia ( $\mathrm{x}$-axis) and SLEs on continuous measures of mental health and physical health (y-axis). Raw data associations between polygenic risk score for schizophrenia and baseline health outcome by recent SLEs are visualized as scatter. 


\section{Sensitivity analyses}

The sensitivity analyses provided support for the robustness of the findings. Analyses with previous SLEs (at $\mathrm{t}-1$ ) showed similar results to those with recent SLEs (Table 3), with the exception of the interaction between previous SLEs and ES-SCZ on physical health, which was not nominally statistically significant. Estimates for previous SLEs were generally lower than those of recent SLEs. The sequential sensitivity analyses replacing independent variables one at a time with the sensitivity measures (PRS-SCZ $Z_{0.1}$, PRS-MDD, ES-SCZ without cannabis use history, tSLEs, dSLEs, iSLEs) corroborated previous findings (eTable 7-11). In comparison to recent iSLEs, the estimates for the interaction between ES-SCZ and dSLEs were larger and remained nominally statistically significant. The iteration of the primary analyses using the narrow dimensions of mental and physical health outcomes produced results similar to the primary analysis (Table 4).

\section{Discussion}

In this prospective study, we tested the moderating role of genetic and environmental liability on the association between recent SLEs and health outcomes in the general population. Our results illustrate that recent SLEs along with environmental (ES-SCZ) and genetic (PRSSCZ) liability to schizophrenia were associated with poor mental health at the population level. ES-SCZ and SLEs were independently and interactively associated with health outcomes, particularly mental health. Although PRS-SCZ was associated with poorer mental health, the association between PRS-SCZ and physical health was trend-significant after adjusting for age, sex, and education. PRS-SCZ did not moderate the association between SLEs and health outcome. The current findings were confirmed by the converging results obtained from sensitivity analyses. 


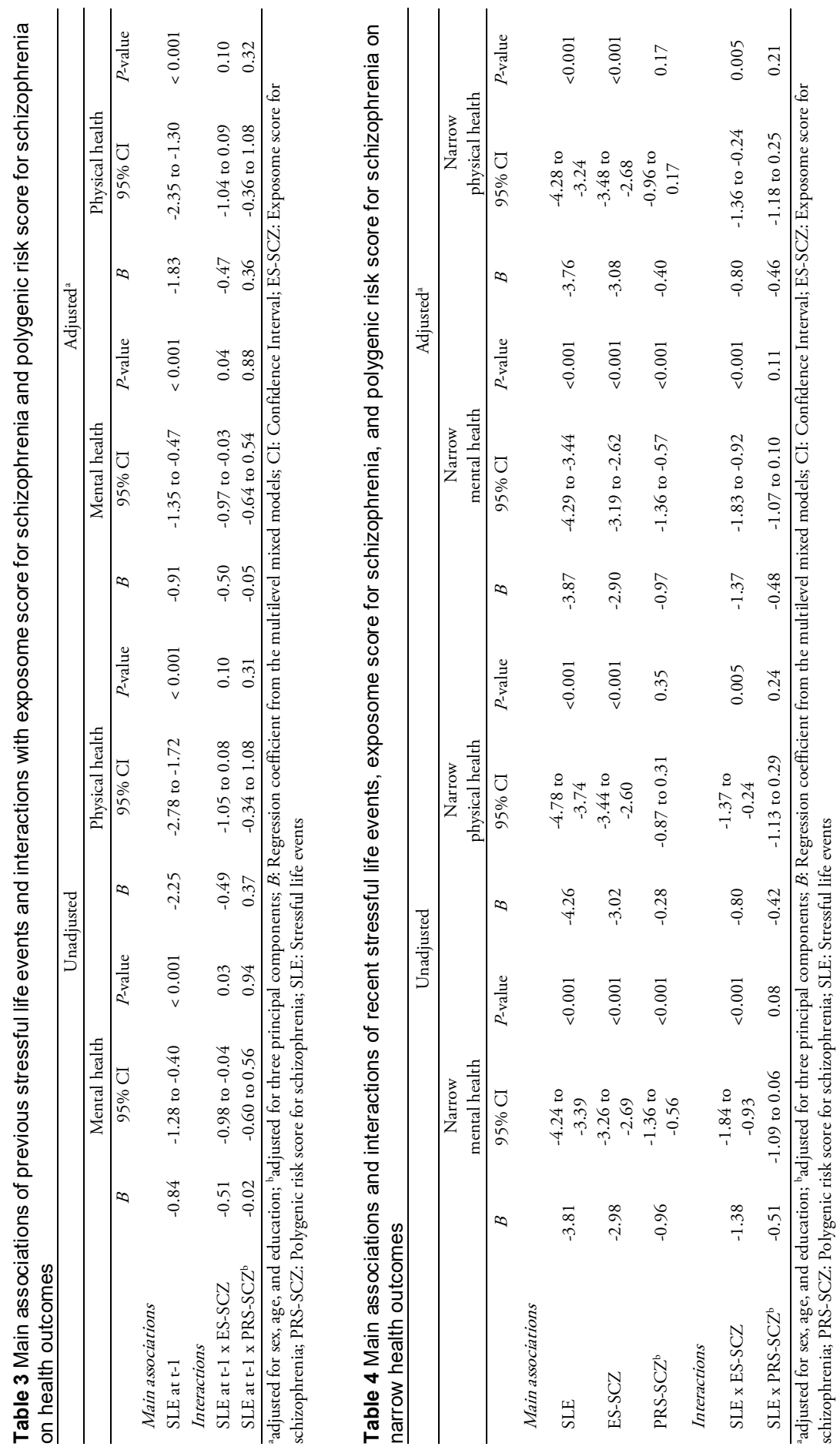


This study adds valuable knowledge to the literature on the longitudinal association between SLE and mental health. Our findings show that recent SLE and environmental liability (ESSCZ) in the general population are associated with poorer mental and physical health. This aligns with previous findings showing that environmental exposures pleiotropically associate with psychosis spectrum disorder ${ }^{21,23,65}$. Supporting the notion of a temporal association between SLEs and health, the analyses with previous SLEs yielded a similar association pattern to those of recent SLEs. However, estimates were higher when investigating the more recent SLEs, indicating that proximity influences the association of SLEs with the outcome $e^{15,66,67}$. Further, along the lines of the diathesis-stress model ${ }^{14}$, we demonstrated that the exposomic liability moderates susceptibility to SLEs later in life. This is in accordance with accumulating evidence indicating that early environmental exposures moderate the association between SLEs later in life and multidimensional psychopathology ${ }^{6,15-19}$. The results show that the network of early and late environmental exposures needs to be elucidated to gain insight into the etiological basis of mental ill-health.

Our study reports novel findings on the association between PRS-SCZ and mental health in a prospective general population sample. While the literature shows inconsistent results for the association between PRS-SCZ and multidimensional psychosis spectrum phenotype (e.g., psychosis expression and negative symptoms $)^{39,41-44,68-70}$, the current results suggest that genetic liability to schizophrenia is pleiotropically associated with broad psychopathology $39,41,42,71,72$.

We did not find a moderating effect of PRS-SCZ on the association between SLEs later in life and health outcomes. This finding is in line with several previous studies investigating the interaction between PRS-MDD and SLEs on depression ${ }^{73,74}$ and our previous findings showing that the association of daily life stressors with positive affect, negative affect, and psychosis proneness at population level was not moderated by PRS-SCZ ${ }^{44}$. However, a recent line of research showed that PRS-MDD moderated the association between SLEs and depression risk ${ }^{32-34}$. Given the inconsistencies, further research is needed to understand whether PRS-SCZ moderates stress-sensitivity.

Some limitations should be acknowledged. Although our exposome score estimation conformed to the approach validated in a large international study, exposome score was limited by the degree to which the exposures that were used in the original model were reliably assessed in the current sample, and thereby lacking other exposures that might be of relevance (e.g., obstetric and pregnancy complications) ${ }^{75}$. Although participants were asked to report past SLEs and current health outcomes, differences in the estimates of iSLE and 
dSLE demonstrate that the reporting of SLEs might be influenced by current mental and physical health. Notwithstanding, the analyses using previous SLEs provide some support for temporal association. Further, although our analysis revealed nominally statistically significant associations of PRS-SCZ with mental health, larger samples may be required to detect a gene-environment interaction in the general population given smaller effect sizes.

In conclusion, we have shown that the genetic and environmental liability for schizophrenia contribute to broad mental health outcomes at the population level. Our findings showing that the network of antecedent environmental liability increases the vulnerability to stressful events later in life provide further support to the diathesis-stress theory. From a clinical perspective, our results suggest that the integration of SLEs can increase the clinical utility of electronic health records. From a public health perspective, our findings underline the promise for investment in public health strategies that focus on modifiable environmental factors over the life span to improve population-based mental health outcomes.

\section{Acknowledgement}

\section{Author Conflict of Interest Disclosures}

Dr O'Donovan is supported by a collaborative research grant from Takeda Pharmacuticals. Takeda played no part in the conception, design, implementation, funding or interpretation of this paper. No other disclosures were reported.

\section{Funding/Support}

NEMESIS-2 is conducted by the Netherlands Institute of Mental Health and Addiction (Trimbos Institute) in Utrecht. Financial support has been received from the Ministry of Health, Welfare and Sport, with supplementary support from the Netherlands Organization for Health Research and Development (ZonMw). This work was supported by the European Community's Seventh Framework Program under grant agreement No. HEALTH-F22009-241909 (Project EU-GEI). Dr O'Donovan is supported by MRC programme grant (G08005009) and an MRC Centre grant (MR/L010305/1). Dr Rutten was funded by a VIDI award number 91718336 from the Netherlands Scientific Organisation. Drs Guloksuz and van Os are supported by the Ophelia research project, ZonMw grant number: 636340001 .

\section{Role of funder/sponsor statement}

The funder had no role in the design and conduct of the study; collection, management, analysis, and interpretation of the data; preparation, review, or approval of the manuscript; and decision to submit the manuscript for publication. 
Access to data and data analyses

Pries and Dr Guloksuz had full access to all the data in the study and take responsibility for the integrity of the data and the accuracy of the data analysis. 


\section{References}

1. Belbasis L, Kohler CA, Stefanis N, et al. Risk factors and peripheral biomarkers for schizophrenia spectrum disorders: an umbrella review of metaanalyses. Acta Psychiatr Scand. 2018;137(2):88-97.

2. Thoits PA. Stress and health: major findings and policy implications. J Health Soc Behav. 2010;51 Suppl(1_suppl):S41-53.

3. Cohen S, Murphy MLM, Prather AA. Ten Surprising Facts About Stressful Life Events and Disease Risk. Annu Rev Psychol. 2019;70:577-597.

4. Ludwig L, Pasman JA, Nicholson T, et al. Stressful life events and maltreatment in conversion (functional neurological) disorder: systematic review and meta-analysis of case-control studies. Lancet Psychiatry. 2018;5(4):307-320.

5. de Graaf R, Bijl RV, ten Have M, Beekman AT, Vollebergh WA. Rapid onset of comorbidity of common mental disorders: findings from the Netherlands Mental Health Survey and Incidence Study (NEMESIS). Acta Psychiatr Scand. 2004;109(1):55-63.

6. McLaughlin KA, Conron KJ, Koenen KC, Gilman SE. Childhood adversity, adult stressful life events, and risk of past-year psychiatric disorder: a test of the stress sensitization hypothesis in a populationbased sample of adults. Psychol Med. 2010;40(10):1647-1658.

7. Kivimäki M, Steptoe A. Effects of stress on the development and progression of cardiovascular disease. Nature Reviews Cardiology. 2018;15(4):215.

8. Michl LC, McLaughlin KA, Shepherd K, NolenHoeksema S. Rumination as a Mechanism Linking Stressful Life Events to Symptoms of Depression and Anxiety: Longitudinal Evidence in Early Adolescents and Adults. J Abnorm Psychol. 2013;122(2):339-352.

9. Norhayati M, Hazlina NN, Asrenee A, Emilin WWJJoaD. Magnitude and risk factors for postpartum symptoms: a literature review. 2015;175:34-52.

10. Ono K, Takaesu Y, Nakai Y, et al. Associations among depressive symptoms, childhood abuse, neuroticism, and adult stressful life events in the general adult population. Neuropsychiatric disease treatment. 2017;13:477.

11. de Graaf R, ten Have M, Tuithof M, van Dorsselaer S. First-incidence of DSM-IV mood, anxiety and substance use disorders and its determinants: results from the Netherlands Mental Health Survey and Incidence Study-2. Journal of affective disorders. 2013;149(1-3):100-107.
12. Weissman MM, Pathak J, Talati A. Personal Life Events-A Promising Dimension for Psychiatry in Electronic Health Records. JAMA Psychiatry. 2019;77(2):115-116.

13. Myers B, McLaughlin KA, Wang S, Blanco C, Stein D. Associations between childhood adversity, adult stressful life events, and past-year drug use disorders in the National Epidemiological Study of Alcohol and Related Conditions (NESARC). Psychology of Addictive Behaviors. 2014;28(4):1117.

14. Pruessner M, Cullen AE, Aas M, Walker EF. The neural diathesis-stress model of schizophrenia revisited: An update on recent findings considering illness stage and neurobiological and methodological complexities. Neurosci Biobehav Rev. 2017;73:191-218.

15. Mansueto G, Faravelli C. Recent life events and psychosis: The role of childhood adversities. Psychiatry Res. 2017;256:111-117.

16. Holtzman CW, Trotman HD, Goulding SM, et al. Stress and neurodevelopmental processes in the emergence of psychosis. Neuroscience. 2013;249:172-191

17. Kendler KS, Kuhn JW, Prescott CA. Childhood sexual abuse, stressful life events and risk for major depression in women. Psychological medicine. 2004;34(8):1475-1482.

18. Morgan C, Reininghaus $U$, Fearon $P$, et al. Modelling the interplay between childhood and adult adversity in pathways to psychosis: initial evidence from the AESOP study. Psychological Medicine. 2014;44(2):407-419.

19. Ayesa-Arriola R, Setien-Suero E, Marques-Feixa L, et al. The synergetic effect of childhood trauma and recent stressful events in psychosis: associated neurocognitive dysfunction. Acta Psychiatr Scand. 2020;141(1):43-51.

20. Lataster J, Myin-Germeys I, Lieb R, Wittchen HU, Van Os J. Adversity and psychosis: a 10-year prospective study investigating synergism between early and recent adversity in psychosis. Acta Psychiat Scand. 2012;125(5):388-399.

21. ten Have M, de Graaf R, Van Dorsselaer S, Tuithof M, Kleinjan M, Penninx BW. Childhood maltreatment, vulnerability characteristics and adult incident common mental disorders: 3-year longitudinal data among $>10,000$ adults in the general population. Journal of psychiatric research. 2019;113:199-207. 
22. Beards S, Gayer-Anderson C, Borges S, Dewey ME, Fisher HL, Morgan C. Life events and psychosis: a review and meta-analysis. Schizophr Bull. 2013;39(4):740-747.

23. Barzilay R, Calkins ME, Moore TM, et al. Association between traumatic stress load, psychopathology, and cognition in the Philadelphia Neurodevelopmental Cohort. Psychological medicine. 2019;49(2):325-334.

24. Suliman S, Mkabile SG, Fincham DS, Ahmed R, Stein DJ, Seedat S. Cumulative effect of multiple trauma on symptoms of posttraumatic stress disorder, anxiety, and depression in adolescents. Compr Psychiatry. 2009;50(2):121-127.

25. Evans GW, Li D, Whipple SS. Cumulative risk and child development. Psychol Bull. 2013;139(6):1342-1396.

26. Karam EG, Friedman MJ, Hill ED, et al. Cumulative traumas and risk thresholds: 12-month PTSD in the World Mental Health (WMH) surveys. Depress Anxiety. 2014;31(2):130-142.

27. Guloksuz S, van Nierop M, Lieb R, van Winkel R, Wittchen HU, van Os J. Evidence that the presence of psychosis in non-psychotic disorder is environment-dependent and mediated by severity of non-psychotic psychopathology. Psychological Medicine. 2015;45(11):2389-2401.

28. Gottesman, II, Shields J. A polygenic theory of schizophrenia. Proceedings of the National Academy of Sciences of the United States of America. 1967;58(1):199-205.

29. Guloksuz S, Pries LK, Delespaul P, et al. Examining the independent and joint effects of molecular genetic liability and environmental exposures in schizophrenia: results from the EUGEI study. World Psychiatry. 2019;18(2):173-182.

30. Mas S, Boloc D, Rodriguez N, et al. Examining Gene-Environment Interactions Using Aggregate Scores in a First-Episode Psychosis Cohort. Schizophr Bull. 2020.

31. Bernardo M, Bioque M, Cabrera B, et al. Modelling gene-environment interaction in first episodes of psychosis. Schizophr Res. 2017;189:181-189.

32. Colodro-Conde L, Couvy-Duchesne B, Zhu G, et al. A direct test of the diathesis-stress model for depression. Molecular psychiatry. 2018;23(7):1590.

33. Arnau-Soler A, Adams MJ, Clarke TK, et al. A validation of the diathesis-stress model for depression in Generation Scotland. Transl Psychiatry. 2019;9(1):25.

34. Arnau-Soler A, Macdonald-Dunlop E, Adams MJ, et al. Genome-wide by environment interaction studies of depressive symptoms and psychosocial stress in UK Biobank and Generation Scotland. Transl Psychiatry. 2019;9(1):14.

35. Zheutlin AB, Dennis J, Karlsson Linner R, et al. Penetrance and Pleiotropy of Polygenic Risk Scores for Schizophrenia in 106,160 Patients Across Four Health Care Systems. The American journal of psychiatry. 2019;176(10):846-855.

36. Kember RL, Merikangas AK, Verma SS, et al. Polygenic risk of psychiatric disorders exhibits cross-trait associations in electronic health record data. bioRxiv. 2019:858027.

37. The Brainstorm Consortium, Anttila V, BulikSullivan B, et al. Analysis of shared heritability in common disorders of the brain. Science. 2018;360(6395):eaap8757.

38. Richardson TG, Harrison S, Hemani G, Davey Smith G. An atlas of polygenic risk score associations to highlight putative causal relationships across the human phenome. Elife. 2019;8:e43657.

39. Mistry S, Harrison JR, Smith DJ, Escott-Price V, Zammit $S$. The use of polygenic risk scores to identify phenotypes associated with genetic risk of schizophrenia: Systematic review. Schizophr Res. 2018;197:2-8.

40. Pazoki R, Lin BD, van Eijk KR, et al. Polygenic risk scores are associated with quality of life in schizophrenia. submitted.

41. Jones HJ, Stergiakouli E, Tansey KE, et al. Phenotypic Manifestation of Genetic Risk for Schizophrenia During Adolescence in the General Population. JAMA psychiatry. 2016;73(3):221-228.

42. van Os J, van der Steen Y, Islam MA, et al. Evidence that polygenic risk for psychotic disorder is expressed in the domain of neurodevelopment, emotion regulation and attribution of salience. Psychol Med. 2017;47(14):2421-2437.

43. van Os J, Pries LK, Delespaul P, et al. Replicated evidence that endophenotypic expression of schizophrenia polygenic risk is greater in healthy siblings of patients compared to controls, suggesting gene-environment interaction. The EUGEI study. Psychol Med. 2019;113:1-14.

44. Pries LK, Klingenberg B, Menne-Lothmann C, et al. Polygenic liability for schizophrenia and childhood adversity influences daily-life emotion dysregulation and psychosis proneness. Acta Psychiatr Scand. 2020:778761.

45. Kendler KS. From many to one to many- the search for causes of psychiatric illness. JAMA psychiatry. 2019;76(10):1085-1091.

46. Pries LK, Lage-Castellanos A, Delespaul P, et al. Estimating Exposome Score for Schizophrenia Using Predictive Modeling Approach in Two 
Independent Samples: The Results From the EUGEI Study. Schizophr Bull. 2019;45(5):960965.

47. de Graaf R, Ormel J, ten Have M, Burger H, BuistBouwman M. Mental disorders and service use in The Netherlands. Results from the European Study of the Epidemiology of Mental Disorders (ESEMeD). New York, USA: Cambridge University Press; 2008.

48. The ESEMeD/MHEDEA 2000 Investigators (Alonso J, et al.). Sampling and methods of the European Study of the Epidemiology of Mental Disorders (ESEMeD) project. Acta Psychiatrica Scandinavica. 2004;109:8-20.

49. de Graaf R, Ten Have M, van Dorsselaer S. The Netherlands Mental Health Survey and Incidence Study-2 (NEMESIS-2): design and methods. Int J Methods Psychiatr Res. 2010;19(3):125-141.

50. de Graaf R, ten Have M, van Gool C, van Dorsselaer S. Prevalence of mental disorders and trends from 1996 to 2009. Results from the Netherlands Mental Health Survey and Incidence Study-2. Soc Psychiatry Psychiatr Epidemiol. 2012;47(2):203-213.

51. Nuyen J, Tuithof M, de Graaf R, van Dorsselaer S, Kleinjan M, Have MT. The bidirectional relationship between loneliness and common mental disorders in adults: findings from a longitudinal population-based cohort study. Soc Psychiatry Psychiatr Epidemiol. 2019:1-14.

52. Brugha $\mathrm{T}$, Bebbington $\mathrm{P}$, Tennant $\mathrm{C}$, Hurry $\mathrm{J}$. The List of Threatening Experiences: a subset of 12 life event categories with considerable long-term contextual threat. Psychol Med. 1985;15(1):189194.

53. Pries LK, Guloksuz S, Ten Have M, et al. Evidence That Environmental and Familial Risks for Psychosis Additively Impact a Multidimensional Subthreshold Psychosis Syndrome. Schizophr Bull. 2018;44(4):710-719.

54. Radhakrishnan R, Guloksuz S, Ten Have M, et al. Interaction between environmental and familial affective risk impacts psychosis admixture in states of affective dysregulation. Psychol Med. 2019;49(11):1879-1889.

55. Stewart AL, Ware JE. Measuring functioning and well-being: the medical outcomes study approach. duke university Press; 1992.

56. Ware J, Kosinski M, Keller S. SF-36 physical and mental health summary scales. a user's manual. 2001:1994.

57. HÅvard LJ, Kaasa S. Short form 36 (SF-36) health survey: normative data from the general Norwegian population. Scandinavian journal of social medicine. 1998;26(4):250-258.

58. Schizophrenia Working Group of the Psychiatric Genomics Consortium. Biological insights from 108 schizophrenia-associated genetic loci. Nature. 2014;511(7510):421-427.

59. Stata Statistical Software [computer program] College Station, TX: StataCorp LLC; 2017.

60. Gibbons RD, Hedeker D, Elkin I, et al. Some conceptual and statistical issues in analysis of longitudinal psychiatric data: Application to the NIMH Treatment of Depression Collaborative Research Program dataset. 1993;50(9):739-750.

61. Schuurman NK. Multilevel autoregressive modeling in psychology: Snags and solutions, Utrecht University; 2016.

62. Keller MC. Genex environment interaction studies have not properly controlled for potential confounders: the problem and the (simple) solution. Biological psychiatry. 2014;75(1):18-24.

63. Royston P, White IR. Multiple Imputation by Chained Equations (MICE): Implementation in Stata. J Stat Softw. 2011;45(4):1-20.

64. Rubin DB. Multiple imputation for nonresponse in surveys. Vol 81: John Wiley \& Sons; 2004.

65. Hailes HP, Yu R, Danese A, Fazel S. Long-term outcomes of childhood sexual abuse: an umbrella review. Lancet Psychiatry. 2019;6(10):830-839.

66. Croft J, Heron J, Teufel C, et al. Association of Trauma Type, Age of Exposure, and Frequency in Childhood and Adolescence With Psychotic Experiences in Early Adulthood. JAMA Psychiatry. 2019;76(1):79-86.

67. Morgan C, Reininghaus U, Reichenberg A, et al. Adversity, cannabis use and psychotic experiences: evidence of cumulative and synergistic effects. Brit J Psychiat. 2014;204(5):346-353.

68. Zammit S, Hamshere M, Dwyer S, et al. A population-based study of genetic variation and psychotic experiences in adolescents. Schizophr Bull. 2014;40(6):1254-1262.

69. Sieradzka D, Power RA, Freeman D, et al. Are genetic risk factors for psychosis also associated with dimension-specific psychotic experiences in adolescence? PloS one. 2014;9(4):e94398.

70. Legge SE, Jones HJ, Kendall KM, et al. Association of Genetic Liability to Psychotic Experiences With Neuropsychotic Disorders and Traits. JAMA psychiatry. 2019;76(12):1256-1265.

71. Riglin L, Collishaw S, Richards A, et al. Schizophrenia risk alleles and neurodevelopmental outcomes in childhood: a population-based cohort study. Lancet Psychiat. 2017;4(1):57-62. 
72. Nivard MG, Gage SH, Hottenga JJ, et al. Genetic Overlap Between Schizophrenia and

Developmental Psychopathology: Longitudinal and Multivariate Polygenic Risk Prediction of Common Psychiatric Traits During Development. Schizophr Bull. 2017;43(6):1197-1207.

73. Mullins N, Power RA, Fisher HL, et al. Polygenic interactions with environmental adversity in the aetiology of major depressive disorder. Psychol Med. 2016;46(4):759-770.
74. Musliner KL, Seifuddin F, Judy JA, Pirooznia M, Goes FS, Zandi PP. Polygenic risk, stressful life events and depressive symptoms in older adults: a polygenic score analysis. Psychol Med. 2015;45(8):1709-1720.

75. Garcia-Rizo C, Bitanihirwe BKY. Implications of early life stress on fetal metabolic programming of schizophrenia: A focus on epiphenomena underlying morbidity and early mortality. Prog Neuropsychopharmacol Biol Psychiatry. 2020;101:10991. 


\section{Supplementary material}

\section{eMethod}

The estimation of exposome score for schizophrenia

By using our previously validated estimates for constructing cumulative environmental load in a Dutch cohort (GROUP) ${ }^{1}$, we generated the exposome score for schizophrenia (ES-SCZ) by summing log odds weighted environmental exposures (each exposure defined as absent $=$ " 0 " and present $=" 1$ ") at baseline including cannabis use, hearing impairment, winter-birth, and five childhood adversity domains (sexual, physical and psychological abuse along with emotional neglect and bullying) ${ }^{1}$. A total of eight exposures were used for estimating ES-SCZ. The definition of each of the exposure is provided below.

Childhood adversity domains

Childhood adversity domains were assessed at baseline (T0) across 5 domains before age 16) using a questionnaire based on the NEMESIS-1 trauma questionnaire ${ }^{2}$. Subjects were asked whether they experienced emotional neglect, psychological abuse, peer victimization, physical abuse or sexual abuse. Each of the five childhood adversity domains was binary coded (absent = "0" and present $=$ " $1 "$ ).

Cannabis Exposure

Cannabis use was assessed with the section Illegal Substance Use of the CIDI 3.0 at baseline (T0; lifetime). If subjects reported cannabis use, they were rated on frequency of use in the period of most frequent use on a scale of 1 (never) to 7 (every day). Consistent with previous work ${ }^{3,4}$, a binary variable (absent = "0" and present = " 1 ") was constructed by using the cut-off value of once per week or more in the period most frequent use.

Hearing impairment

Baseline hearing impairment was based on self-reported hearing impairment in the last 12 months (absent = "0" and present = " 1 ").

\section{Winter-birth}

In accordance with previous studies investigating the association between season of birth and schizophrenia in the Northern hemisphere sites ${ }^{5}$, the high-risk birth period was defined based on the winter solstice (December-March), and a binary winter-birth exposure was constructed (absent $=$ " 0 " and present $=" 1 "$ ).

Genotyping procedures, quality control, imputation, and polygenic risk score (PRS) calculation

A. Target Genotype Data Processing

1. Genotyping procedures and quality control steps before imputation.

NEMESIS-2 samples were genotyped on an IPMCN chip (Institute of Psychological Medicine and Clinical Neurology, Cardiff University), which was custom-made for EUGEI (588,628 genotyped variants for 4,043 participants). ${ }^{6}$ Quality control (QC) was done using PLINK v1.9 ${ }^{7}$ as follows. There were 3,861 samples matching with phenotypes. Single nucleotide polymorphisms (SNPs) and samples with call rates below 95\% and 98\%, respectively, were removed. A strict SNP QC only for subsequent sample QC steps was conducted. This involved a minor allele frequency (MAF) threshold $>10 \%$ and a Hardy-Weinberg equilibrium (HWE) $P$-value $>10^{-5}$, followed by linkage disequilibrium (LD) based SNP pruning $\left(\mathrm{R}^{2}<0.5\right)$. This resulted in $-60 \mathrm{~K}$ SNPs to assess sex errors $(\mathrm{n}=145)$, heterozygosity $(\mathrm{F}<5 \mathrm{xSD}$ the standard deviation $(\mathrm{SD}), \mathrm{n}=73)$, and relatedness by pairwise identity by descent $(\mathrm{IBD})$ values $>0.1(\mathrm{n}=170)$. Genetic outliers $(\mathrm{n}=154)$ were identified by principal component analysis (PCA, see below). In total, 3,104 individuals passed these QC steps. After removing failing samples $(\mathrm{n}=757)$, a regular SNP QC was performed (SNP call rate $>95 \%$, HWE $p>1 \mathrm{e}-06, \mathrm{MAF}>0.16 \%$; as the IPMCN chip contains many rare variants, half the SNPs would have been removed if we had applied MAF> $1 \%$; therefore, we loosened MAF threshold to $0.16 \%=10 /\left(2^{*}\right.$ sample size of 3,104$\left.)\right)$. Next, strand ambiguous SNPs and duplicate SNPs were removed, resulting in a total of 298,104 genotyped variants.

2. Imputation on Michigan server.

The QC-ed dataset was chunked by chromosome, and then converted into *.VCF files. The Michigan server was used for imputation with the following settings: reference panel as HRC R1.1 2016; phasing as Eagle v2.3; population as European; model as QC \& imputation. The imputation resulted in 47,101,073 single nucleotide polymorphisms (SNPs). The general imputation quality is shown in eFigure 1. 


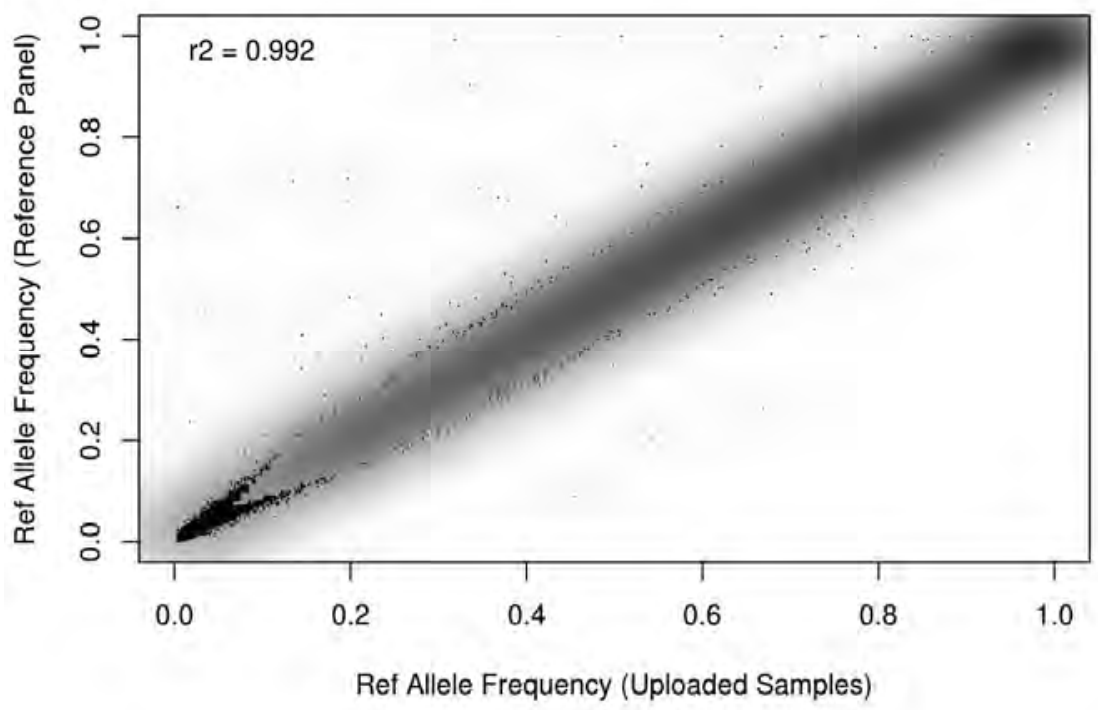

3. Quality control after imputation.

Poor quality SNPs were excluded: multi-allelic SNPs, SNPs with a minor allele frequency $(\mathrm{MAF})<0.0016$ or $\mathrm{INFO}<0.3$, and strand ambiguous AT/CG SNPs. The VCF dosage files were converted into PLINK format dosage files. Finally, 10,356,437 SNPs and 3,104 individuals remained. For hard call (best guess) genotypes, an additional SNP QC (INFO>0.8 and HWE $>10-6$ ) was performed, resulting in 6,436,459 SNPs.

4. Principle components analyses (PCA).

The principal components (PCs) analyses within NEMESIS-2 samples, and NEMESIS- 2 samples along with Hapmap $3^{8}$ populations were conducted by EIGENSTRAT ${ }^{9}$. A strict selection for SNPs with overlap with Hapmap3 SNPs was conducted: 1 . MAF>0.05 and HWE $>0.001 ; 2$. Removal of 24 long LD regions (eTable 1); 3. LD pruning with an $\mathrm{R}^{2}$ of 0.5 ; which resulted in 40,732 best quality genotyped SNPs used to calculate genetic PCs. PCs were firstly calculated with HapMap3 population to exclude European ethnic outliers: exceeding 5 times the standard deviation of Utah residents with Northern and Western European ancestry from the $\mathrm{CEPH}$ collection (CEU) and Toscani in Italia (TSI) populations for the first 2 PCs (eFigure 2). The first 2 PCs explained $>84.3 \%$ of the total. In total, 141 individuals were excluded from this dataset. Secondly, another PCA was conducted using the same SNPs $(\mathrm{n}=40,732)$ but calculated only in NEMESIS-2. The first 2 PCs explained $>30 \%$ of the total. Another 13 individuals were considered as ethnic outliers by the first 2 PCs exceeding 5 times the standard deviation in the NEMESIS- 2 sample. In the end, 3,104 individuals remained. Compared with the self-report ethnic information, $93.75 \%$ of our ethnic-QCed samples are self-reported as "Dutch". We think some people may have understood the question as: "where were you born?", since non-former colony countries have 0 deviations from genetic QC, while former colonies have substantial deviations. In total, 154 samples were identified as European ethnic outliers and excluded. After post-imputation quality control steps, PCA was conducted using the same SNPs $(\mathrm{n}=40,732)$ within NEMESIS-2 samples $(\mathrm{n}=3,104)$. These PCs were then used as covariates in analyses to correct for population stratification. 
eFigure 2. First 2PCs of NEMESIS-2 samples with Hapmap 3 before exclusion of ethnic outliers

Nemesis PCA with Hapmap3 (Outliers > 5SD)

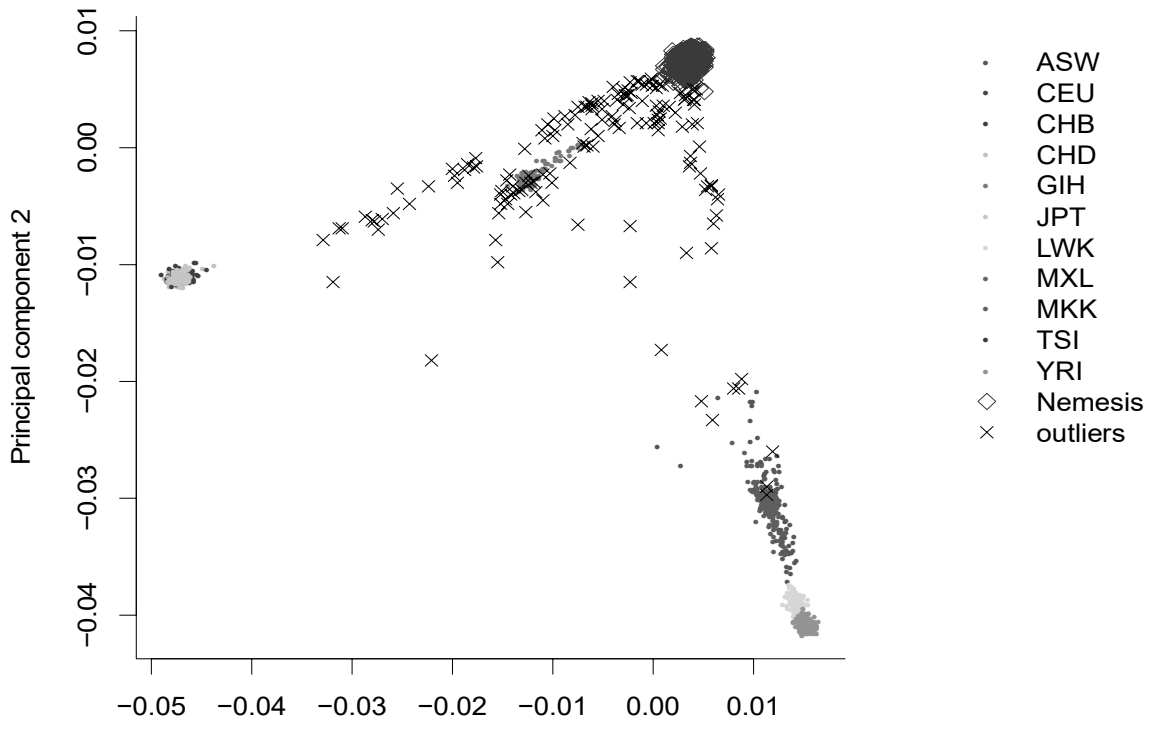

Principal component 1

B. Training schizophrenia GWAS summary statistic processing and calculating polygenic risk score for schizophrenia

We used recent GWASs of schizophrenia ${ }^{10}$ for PRS calculations ${ }^{11}$. As a quality control for PRS calculation, the SNPs that overlapped between the GWASs summary statistics (training datasets) and our dataset were extracted. Then, insertions or deletions, ambiguous SNPs, SNPs with minor allele frequency (MAF) $<0.01$ and imputation quality $\left(\mathrm{R}^{2}\right)<0.8$ in both training and target datasets were excluded. To account for complicated LD structure of SNPs in the genome, these SNPs were clumped in two rounds using PLINK $1.90 \mathrm{~b} 3 \mathrm{z}^{12}$ according to previously established methods ${ }^{13,14}$; round 1 with the default parameters (physical distance threshold $250 \mathrm{~kb}$ and $\mathrm{LD}$ threshold $\left(\mathrm{R}^{2}\right) 0.5$ ); round 2 with a physical distance threshold of 5,000kb and $\mathrm{LD}$ threshold $\left(\mathrm{R}^{2}\right) 0.2$. Additionally, we excluded all SNPs in genomic regions with strong or complex LD structures (e.g. the MHC region on chromosome 6; eTable 1). The odds ratios (ORs) were reported in the summary statistics, and were log-converted to beta values as effect sizes. Sample overlap between NEMESIS-2 data with schizophrenia GWAS cohort (PCG and CLOZUK cohorts) is unlikely since all samples belong to different cohorts. We constructed PRS based on schizophrenia risk alleles weighted by their schizophrenia increasing effect estimate using the Purcell et al. method ${ }^{7,15}$, i.e. using PLINK's score function. PRS was calculated for 3,104 samples (those remaining after QC). Informed by the PGC analyses, PRS for schizophrenia with a significance cut-off $P<0.05$ was used in the main analyses to achieve a balance between the number of false-positive and true-positive risk alleles ${ }^{16}$. 
eTable 1: The complex-LD regions (build GRCh37) removed for PCA analysis

\begin{tabular}{ccc}
\hline Chromosome & Base pair start & Base pair end \\
\hline 6 & 25392021 & 33392022 \\
8 & 111930824 & 114930824 \\
11 & 46043424 & 57243424 \\
1 & 48287980 & 52287979 \\
2 & 86088342 & 101041482 \\
2 & 134666268 & 138166268 \\
2 & 183174494 & 190174494 \\
3 & 47524996 & 50024996 \\
3 & 83417310 & 86917310 \\
3 & 88917310 & 96017310 \\
5 & 44464243 & 50464243 \\
5 & 97972100 & 100472101 \\
5 & 128972101 & 131972101 \\
5 & 135472101 & 138472101 \\
6 & 56892041 & 63942041 \\
6 & 139958307 & 142458307 \\
7 & 55225791 & 66555850 \\
8 & 7962590 & 11962591 \\
8 & 42880843 & 49837447 \\
10 & 36959994 & 43679994 \\
11 & 87860352 & 90860352 \\
12 & 33108733 & 41713733 \\
12 & 111037280 & 113537280 \\
20 & 32536339 & 35066586 \\
\hline & &
\end{tabular}

\section{The sensitivity measures}

Polygenic risk score for schizophrenia at $P$-threshold of $<0.1$

Given that the analyses of PRS-SCZ with a $P$-threshold of $<0.1$ (PRS-SCZ $Z_{0.1}$ ) on the current phenotypes (mental and physical health; eTable 3) showed slightly better performance in this sample, we repeated the analyses with PRS-SCZ 0.1 .

The sum score of adulthood stressful life events

By using nine life events listed in the "Brugha Life events section" ${ }^{17}$ (eTable 2), a sum score of stressful life events (tSLEs) was generated.

The independent and dependent adulthood stressful life events

The recent SLEs were categorized into independent (iSLEs, items 1-4 in the eTable 2) and dependent (dSLEs, items 5-9 in the eTable 2) based on previous studies ${ }^{17-20}$

Polygenic risk score for major depressive disorder

By applying the same methodology as described above for the construction of PRS-SCZ, we generated the polygenic risk score for major depressive disorder (PRS-MDD) at $P$-threshold $0.05^{21}$.

Exposome score for schizophrenia without cannabis use history

We applied the same methodology as described above for the construction of ES-SCZ after leaving cannabis use history out to estimate exposome score for schizophrenia without cannabis use history (ES-SCZ $Z_{\text {(no cannabis) }}$ ) using a total of seven exposures including hearing impairment, winter-birth, and five childhood adversity domains (sexual, physical and psychological abuse along with emotional neglect and bullying). 
Narrow mental and physical health

The Short-Form-36 Health Survey (SF-36) ${ }^{22}$ consists of nine subscales, each scale ranging from poor (0) to good (100) functioning. Based on the Short-Form-36 Health Survey manual ${ }^{23}$, narrow dimensions of mental health (mental health and role limitations due to emotional problems scales) and physical health (physical functioning, role limitation due to physical health problems, and bodily pain) were calculated.

eTable 2 List of stressful life events

\begin{tabular}{lc}
\hline Items & Frequency $^{\mathrm{a}}$ \\
\hline (1) Serious illness or injury to subject & $10.19 \%$ \\
(2) Serious illness or injury to a close relative & $29.20 \%$ \\
(3) Death of first-degree relative including child or spouse & $14.02 \%$ \\
(4) Death of close family friend or second degree relative & $32.76 \%$ \\
(5) Separation due to marital difficulties & $5.45 \%$ \\
(6) Broke off a steady relationship & $6.36 \%$ \\
(7) Serious problem with a close friend, neighbor or relative & $7.31 \%$ \\
(8) Subject sacked from job & $6.98 \%$ \\
(9) Major financial crisis & $5.33 \%$ \\
\hline
\end{tabular}

$\sqrt{\mathrm{a}} \mathrm{Data}$ from surveys of 6646 participants at four time points (T0, T1, T2, and T3), yielding a total of 20,574 observations for analyses in the 'long' format.

eTable 3 Explained variance (R2) of baseline mental and physical health for different polygenic risk score $P$ thresholds and environmental exposures

\begin{tabular}{lcccccc}
\hline & \multicolumn{3}{c}{ Mental health } & \multicolumn{3}{c}{ Physical health } \\
\hline & $B$ & $P$-value & $R 2(\%)$ & $B$ & $P$-value & $R 2(\%)$ \\
$\begin{array}{l}\text { Polygenic risk score for schizophrenia at } \\
\text { different } P \text {-thresholds }\end{array}$ & & & & & & \\
$5 \cdot 10^{-8}$ & & & & & & \\
$5 \cdot 10^{-5}$ & -0.32 & 0.19 & 0.17 & -0.26 & 0.40 & 0.55 \\
$5 \cdot 10^{-3}$ & -0.34 & 0.16 & 0.18 & -0.29 & 0.35 & 0.55 \\
0.05 & -0.64 & 0.009 & 0.33 & -0.49 & 0.12 & 0.60 \\
0.1 & -0.85 & $<0.001$ & 0.50 & -0.49 & 0.12 & 0.60 \\
0.5 & -0.86 & $<0.001$ & 0.51 & -0.57 & 0.07 & 0.63 \\
Recent stressful life events & -0.80 & 0.001 & 0.45 & -0.54 & 0.09 & 0.62 \\
Exposome score for schizophrenia & -6.27 & $<0.001$ & 5.04 & -6.83 & $<0.001$ & 3.73 \\
\hline
\end{tabular}

${ }^{a}$ adjusted for three principal components; $B$ : regression coefficient from the regression models at baseline; $R 2$ : Explained variance by R-squared in \% 







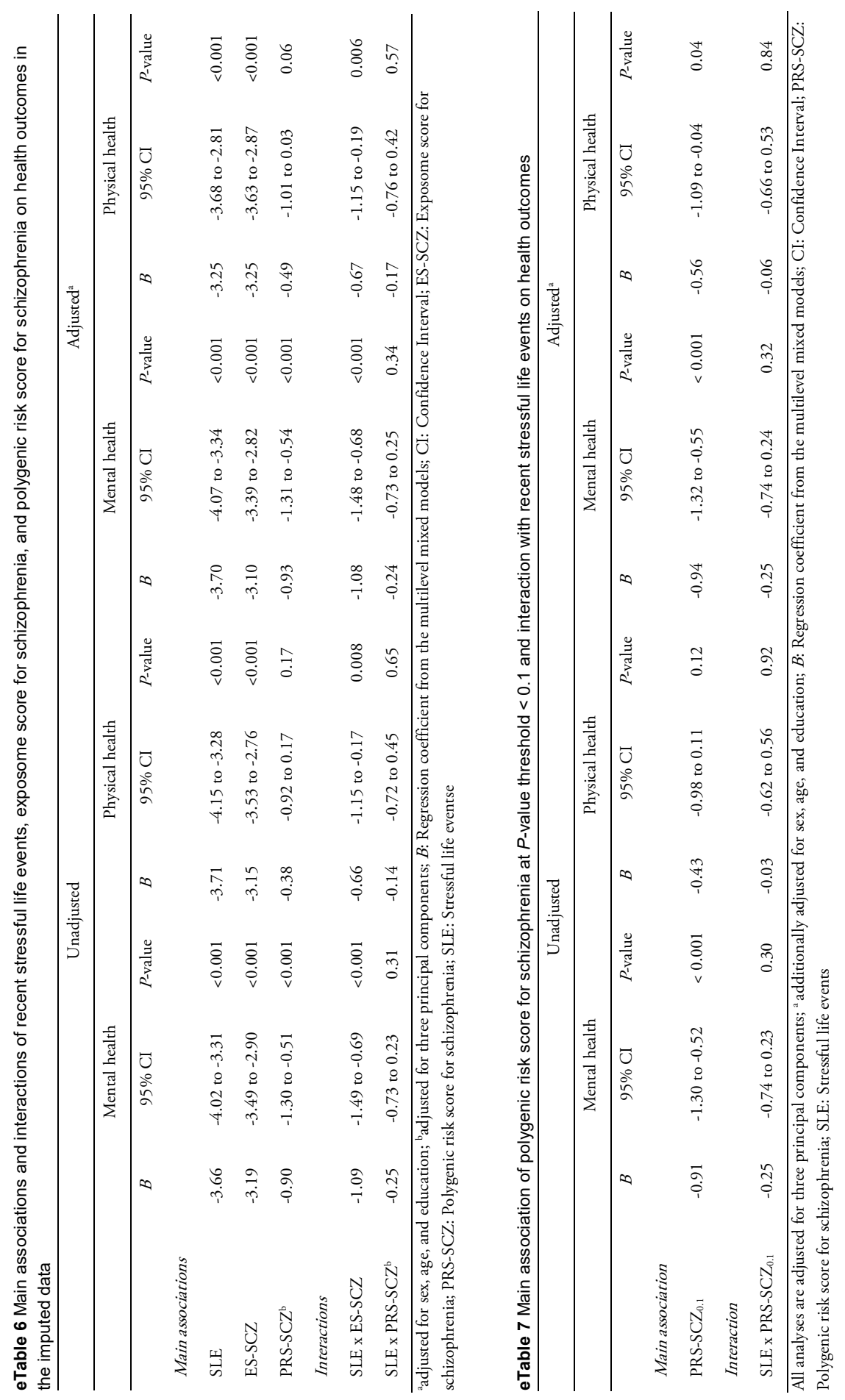




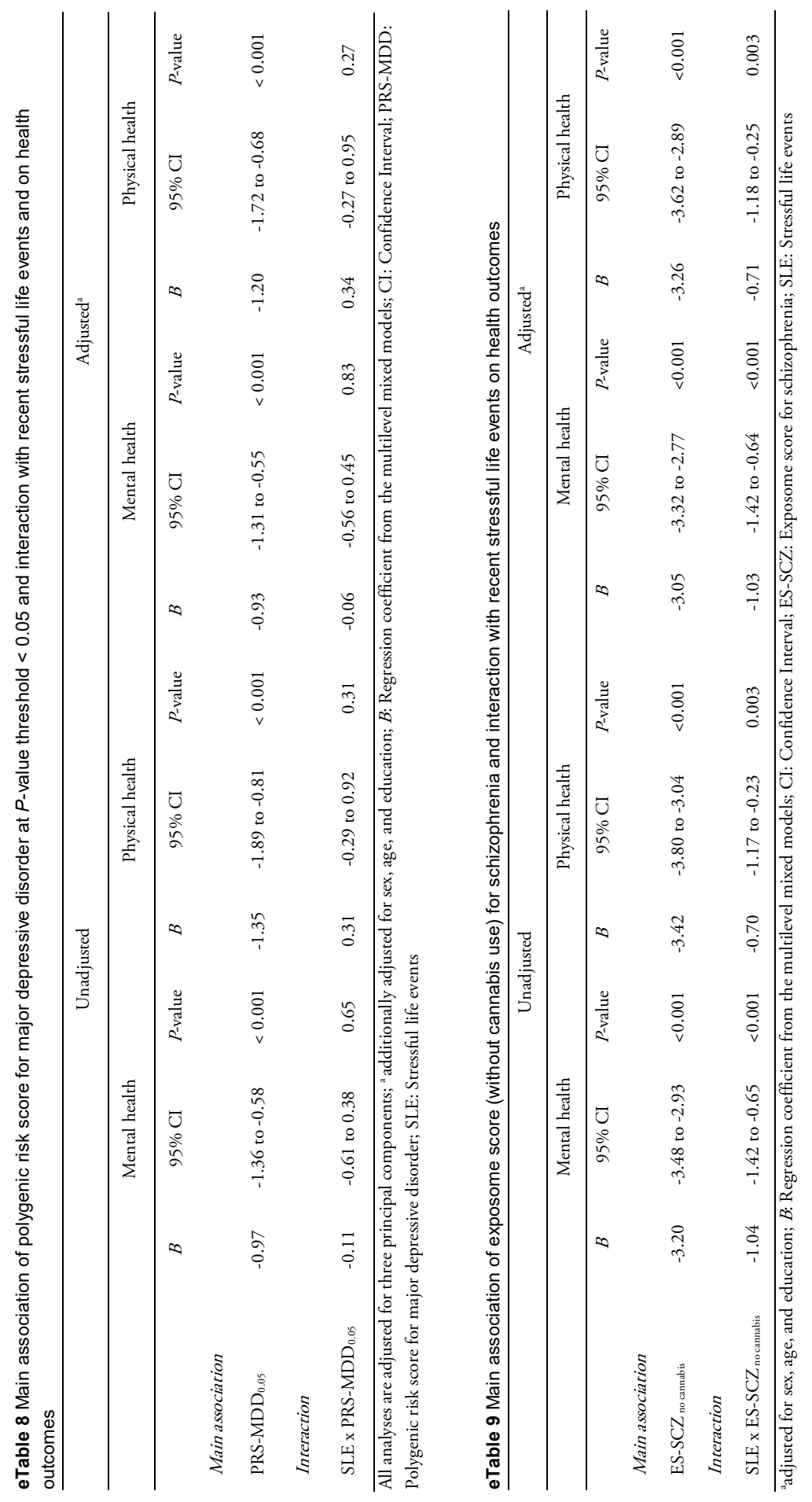




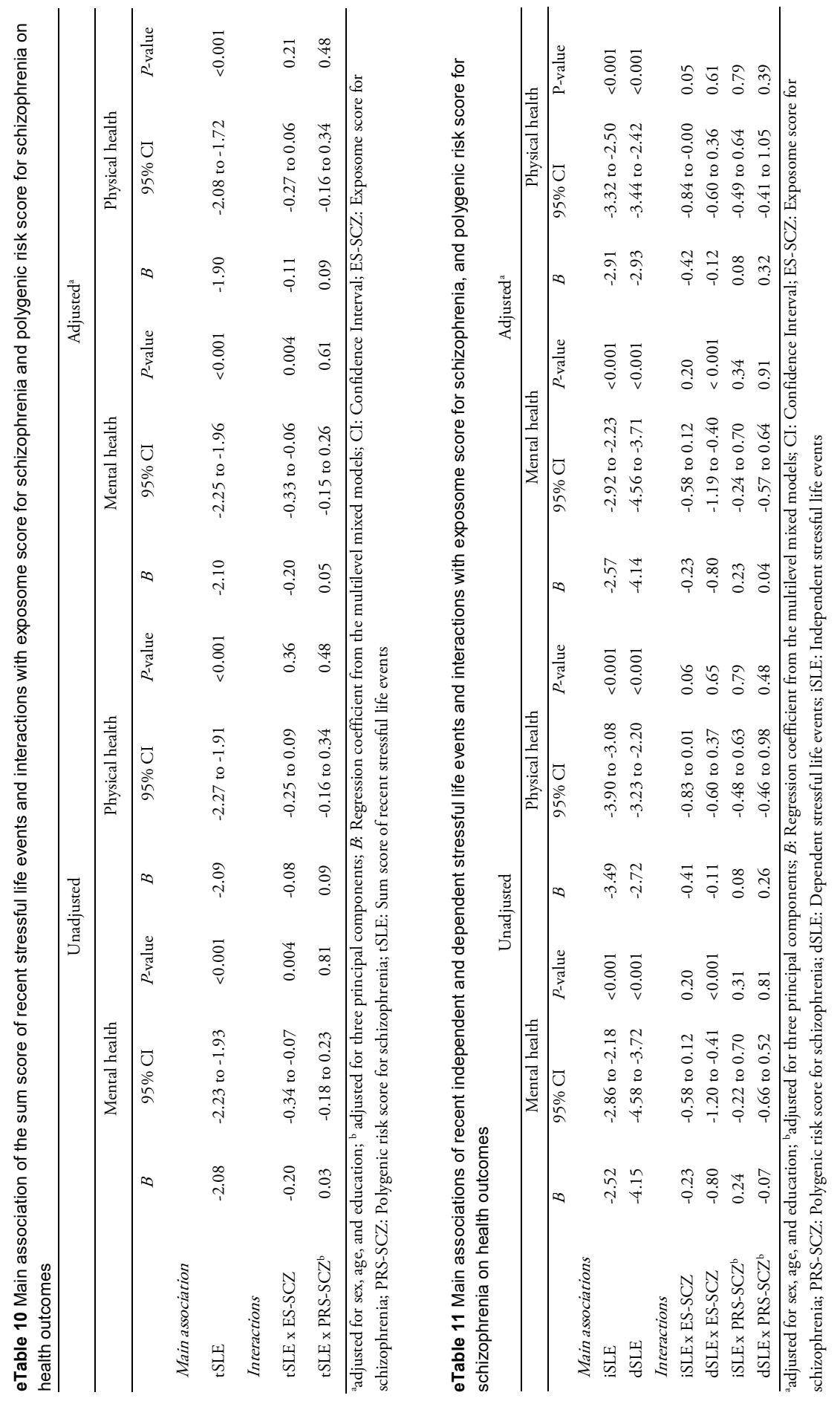




\section{References}

1. Pries LK, Lage-Castellanos A, Delespaul P, et al. Estimating Exposome Score for Schizophrenia Using Predictive Modeling Approach in Two Independent Samples: The Results From the EUGEI Study. Schizophr Bull. 2019;45(5):960965.

2. de Graaf R, Ten Have M, van Dorsselaer S. The Netherlands Mental Health Survey and Incidence Study-2 (NEMESIS-2): design and methods. Int J Methods Psychiatr Res. 2010;19(3):125-141.

3. Pries LK, Guloksuz S, Ten Have M, et al. Evidence That Environmental and Familial Risks for Psychosis Additively Impact a Multidimensional Subthreshold Psychosis Syndrome. Schizophr Bull. 2018;44(4):710-719.

4. Radhakrishnan R, Guloksuz S, Ten Have M, et al. Interaction between environmental and familial affective risk impacts psychosis admixture in states of affective dysregulation. Psychol Med. 2019;49(11):1879-1889.

5. Davies G, Welham J, Chant D, Torrey EF, McGrath J. A systematic review and meta-analysis of Northern Hemisphere season of birth studies in schizophrenia. Schizophr Bull. 2003;29(3):587593.

6. EUGEI investigators. Identifying gene-environment interactions in schizophrenia: contemporary challenges for integrated, large-scale investigations. Schizophr Bull. 2014;40(4):729-736.

7. Purcell S, Neale B, Todd-Brown K, et al. PLINK: a tool set for whole-genome association and population-based linkage analyses. Am J Hum Genet. 2007;81(3):559-575.

8. HapMap 3 [Internet] [cited 2018 Sep 16] Available from:

https://www.sanger.ac.uk/resources/downloads/hu man/hapmap3.html.

9. Price AL, Patterson NJ, Plenge RM, Weinblatt ME, Shadick NA, Reich D. Principal components analysis corrects for stratification in genome-wide association studies. Nat Genet. 2006;38(8):904909.

10. Pardinas AF, Holmans P, Pocklington AJ, et al. Common schizophrenia alleles are enriched in mutation-intolerant genes and in regions under strong background selection. Nat Genet. 2018;50(3):381-389.

11. Choi SW, Heng Mak TS, O’Reilly PF. A guide to performing Polygenic Risk Score analyses. bioRxiv. 2018.
12. Chang CC, Chow CC, Tellier LC, Vattikuti S, Purcell SM, Lee JJ. Second-generation PLINK: rising to the challenge of larger and richer datasets. Gigascience. 2015;4:7.

13. McLaughlin RL, Schijven D, van Rheenen W, et al. Genetic correlation between amyotrophic lateral sclerosis and schizophrenia. Nat Commun. 2017;8:14774.

14. Schur RR, Schijven D, Boks MP, et al. The effect of genetic vulnerability and military deployment on the development of post-traumatic stress disorder and depressive symptoms. Eur Neuropsychopharmacol. 2019;29(3):405-415.

15. International Schizophrenia Consortium, Purcell SM, Wray NR, et al. Common polygenic variation contributes to risk of schizophrenia and bipolar disorder. Nature. 2009;460(7256):748-752.

16. Wray NR, Lee SH, Mehta D, Vinkhuyzen AAE, Dudbridge F, Middeldorp CM. Research Review: Polygenic methods and their application to psychiatric traits. Journal of Child Psychology and Psychiatry. 2014;55(10):1068-1087.

17. Brugha T, Bebbington P, Tennant C, Hurry J. The List of Threatening Experiences: a subset of 12 life event categories with considerable long-term contextual threat. Psychol Med. 1985;15(1):189194.

18. Arnau-Soler A, Adams MJ, Clarke TK, et al. A validation of the diathesis-stress model for depression in Generation Scotland. Transl Psychiatry. 2019;9(1):25.

19. Colodro-Conde L, Couvy-Duchesne B, Zhu G, et al. A direct test of the diathesis-stress model for depression. Molecular psychiatry. 2018;23(7):1590.

20. Arnau-Soler A, Macdonald-Dunlop E, Adams MJ, et al. Genome-wide by environment interaction studies of depressive symptoms and psychosocial stress in UK Biobank and Generation Scotland. Transl Psychiatry. 2019;9(1):14

21. Wray NR, Ripke S, Mattheisen M, et al. Genomewide association analyses identify 44 risk variants and refine the genetic architecture of major depression. Nat Genet. 2018;50(5):668-681.

22. Stewart AL, Ware JE. Measuring functioning and well-being: the medical outcomes study approach. duke university Press; 1992.

23. Ware J, Kosinski M, Keller S. SF-36 physical and mental health summary scales. a user's manual. 2001:1994 




\section{Abstract}

Background: Psychosis spectrum disorder has a complex pathoetiology characterized by a network of environmental and genetic vulnerabilities. The present study aims to investigate the role of gene-environment interaction using aggregate scores of genetic (polygenic risk score: PRS-SCZ) and environment liability for schizophrenia (exposome score: ES-SCZ) across the psychosis continuum.

Methods: The sample consisted of 1699 patients, 1753 unaffected siblings, and 1542 healthy comparison participants. The Structured Interview for Schizotypy-Revised (SIS-R) was administered to analyze scores of total, positive, and negative schizotypy in siblings and healthy comparison participants. PRS-SCZ was trained using the PGC2 results. Guided by the approach validated in a large international study, ES-SCZ was trained and validated in two independent subsamples of the current dataset.

Results: Both genetic and environmental vulnerability were associated with case-control status. Furthermore, there was evidence for additive interaction between PRS-SCZ and ESSCZ increasing the odds for schizophrenia spectrum diagnosis (relative excess risk due to interaction $=6.79,[95 \% \mathrm{CI}: 3.32,10.26], P<0.001)$. In siblings and healthy comparison participants, PRS-SCZ and ES-SCZ were associated with all SIS-R dimensions and evidence was found for an interaction between PRS-SCZ and ES-SCZ on the total $(B=0.006$ [95\%CI: 0.003, 0.009], $P<0.001)$, positive (B = 0.006 [95\%CI: 0.002, 0.009], $P=0.002$ ), and negative ( $\mathrm{B}=0.006$, [95\%CI: 0.004, 0.009], $P<0.001)$ schizotypy dimension.

Conclusions: The interplay between exposome load and schizophrenia genetic liability contributing to psychosis across the spectrum of expression provide further empirical support to the notion of etiological continuity underlying an extended psychosis phenotype.

Keywords: Genetics, Environment, Psychosis, Schizotypy 


\section{Introduction}

Psychosis spectrum ranges from serious, enduring and disabling illness to transient, subthreshold psychotic experiences in non-clinical populations. ${ }^{1}$ Psychosis spectrum represents with a wide range of symptoms including aberrant thinking and reasoning, perceptual abnormalities, cognitive disturbance, as well as motivational and social deficits. Consistent with the extended psychosis phenotype model, prevalence rates are estimated at $5-8 \%$ for psychotic experiences in the general population, $3 \%$ for clinical psychotic disorders, and $0.5 \%$ for arguably the most severe end of the spectrum, meeting diagnostic criteria for schizophrenia. ${ }^{2}$

The etiological and pathophysiological theories of psychosis spectrum have evolved to encompass genetic and environmental factors and their interaction. ${ }^{3}$ The pattern of familial clustering suggests the presence of genetic factors, with heritability estimates of up to $80 \%$ for schizophrenia and 73\% for the wider phenotype. ${ }^{4}$ More recent molecular genetic studies have confirmed that psychosis spectrum disorder (PSD), as a common complex trait, has a polygenic architecture, which is mainly shaped by many common allele variants with small effect sizes that are normally distributed among the general population. ${ }^{5}$ With the advent of the GWAS approach, the Psychiatric Genomics Consortium has identified 145 significant loci associated with schizophrenia. ${ }^{6}$ It is now possible to calculate an individual score summarizing the level of genetic risk for schizophrenia, known as polygenic risk score for schizophrenia (PRS-SCZ).

Similarly, several environmental exposures have been associated with PSD, such as childhood adversities, cannabis use, urbanicity, migration, ethnic minorities, hearing impairment, and perinatal factors. ${ }^{7,8}$ In accordance with the diathesis-stress model, there is evidence supporting gene-environment interaction in the etiology of schizophrenia. A recent case-control study found evidence for additive interactions between molecular genetic liability for schizophrenia (i.e. PRS-SCZ) and emotional abuse, emotional neglect, sexual abuse, bullying and regular cannabis use, suggesting that a multitude of environmental factors and PRS-SCZ are independently and jointly associated with schizophrenia. ${ }^{9}$ 
To better accommodate the multiplicity of exposures associated with schizophrenia, ${ }^{10}$ a cumulative environmental exposure score for schizophrenia — exposome score (ES-SCZ) was recently designed and validated through predictive modeling approaches in training and validation datasets of two independent cohorts that followed identical measurement methods for environmental exposures. ${ }^{11}$ This summary measure is generated using weighted coefficients derived from a single model to take into account the interdependency of exposures. Therefore, ES-SCZ prevents overestimation of the weights per exposure that are likely to occur when correlations between exposures are ignored, e.g. weighted estimates of individuals exposures from meta-analyses or simple summation of exposures. Recent studies indicate that the ES-SCZ is associated with psychosis risk states ${ }^{12}$ as well as mental and physical health ${ }^{13}$ in the general population.

By leveraging aggregate scores of genetic (PRS-SCZ) and environmental liability (ES-SCZ) in the current study, we aimed to investigate the role of gene-environment interaction across the psychosis continuum in a multinational multicenter sample of patients diagnosed with schizophrenia spectrum disorder, their siblings, and healthy comparison participants.

\section{Methods}

\section{Study population}

Data were derived from the Workpackage 6 (WP6) of the European Network of National Networks studying Gene-Environment Interactions in Schizophrenia (EUGEI) and the Genetic Risk and Outcome for Psychosis (GROUP) studies, collected using uniform assessment schedules between 2010 and 2015 in the Netherlands, Turkey, Spain and Serbia. ${ }^{14,15}$ Both projects were approved by the Medical Ethics Committees of all participating sites and conducted in accordance with the Declaration of Helsinki. All respondents provided written informed consent and, in the case of minors, such consent was also obtained from parents or legal guardian.

EUGEI WP6 ("Vulnerability and Severity") was a cross-sectional study that was specifically conducted to investigate the role of gene-environment interaction of the vulnerability and severity of schizophrenia spectrum disorder and its intermediate phenotypes in a family-based setting. Exclusion criteria for all participants were diagnosis of psychotic disorders due to medical condition, history of head injury with loss of consciousness, and intelligence quotient $<70$. 
GROUP is a naturalistic longitudinal cohort study that started in 2004 in the Netherlands and Dutch-speaking part of Belgium and collected data at baseline, 3 and 6 years follow-ups over an approximate 10-year period, with the aim of studying the interplay of genetic and environmental factors impacting vulnerability and resilience in psychotic disorders. Individuals in the sibling group who manifested lifetime psychotic disorder over the study period were reassigned to the patient group.

Further details of the GROUP and EUGEI projects are provided elsewhere. ${ }^{14,15}$ The current analyses used a merged data of GROUP baseline data and EUGEI WP6 cross-sectional data including 1699 patients, 1753 siblings, and 1542 unrelated healthy comparison participants who were of Caucasian white ethnic origin and had available genotype data.

\section{Outcomes}

\section{Diagnosis of schizophrenia spectrum disorder}

Patients were diagnosed with schizophrenia spectrum disorders according to the DSM-IVTR. The diagnosis was confirmed by the Operational Criteria Checklist for Psychotic and Affective Illness ${ }^{16}$ in EUGEI WP6, and by the Schedules for Clinical Assessment in Neuropsychiatry ${ }^{17}$ and the Comprehensive Assessment of Symptoms and History ${ }^{18}$ in GROUP.

\section{Schizotypy trait}

In both GROUP and EUGEI, the Structured Interview for Schizotypy-Revised (SIS-R) was administered to siblings and healthy comparison participants. The SIS-R is a semi-structured interview containing 20 schizotypal symptoms and 11 schizotypal signs rated on a 4-point scale. ${ }^{19,20}$ Symptoms are defined as verbal responses to standardized questions concerning, for example, magical ideation, illusions, and referential thinking. Signs refer to behaviors that are rated by the interviewer such as goal-directedness of thinking and flatness of affect. Questions and rating procedures are standardized. Guided by previous research, 31 item scores were reduced a priori to two-dimensional scores representing the means of seven positive schizotypy items (covering the areas of referential thinking, psychotic phenomena, derealization, magical ideation, illusions, and suspiciousness) and eight negative / disorganized schizotypy items (covering the areas of social isolation, sensitivity, introversion, restricted affect, disturbances in associative and goal-directed thinking, poverty of speech, and eccentric behavior)..$^{21,22}$ 


\section{Genetic and environmental liability measures}

\section{Exposome score for schizophrenia}

By using our previously validated estimates for constructing cumulative environmental load in this dataset, we generated the ES-SCZ by summing log odds weighted environmental exposures (each exposure defined as absent $=$ " 0 " and present $=$ " 1 ”) including cannabis use, hearing impairment, winter-birth, and childhood adversity domains (emotional and physical neglect, emotional, physical and sexual abuse, and bullying). The definition of each exposure conformed to previous work in this dataset.

Childhood adversities were assessed using the Childhood Trauma Questionnaire Short Form (CTQ). ${ }^{23}$ This consists of 28 items, rated on a 5-point Likert scale, measuring five domains of maltreatment (emotional and physical neglect; emotional, physical and sexual abuse). The psychometric characteristics of the translated versions (Spanish, Turkish, Dutch and Serbian) of the CTQ have been comprehensively studied. ${ }^{24-26}$ To dichotomize each childhood adversity domain ( $0=$ "absent" and $1=$ "present"), consistent with previous work in the EUGEI, ${ }^{9}$ we used the following cut-off scores for each domain: $\geq 9$ for emotional abuse; $\geq 8$ for physical abuse; $\geq 6$ for sexual abuse; $\geq 10$ for emotional neglect; and $\geq 8$ for physical neglect.

Cannabis use was assessed by a modified version of the Cannabis Experiences Questionnaire ${ }^{27}$ in the EUGEI WP6 ( $0=$ "none"; 1="only once or twice"; 2="a few times a year"; 3="a few times a month"; 4="once or more a week"; $5=$ "everyday"), and by the L section of the Composite International Diagnostic Interview (CIDI) $)^{28}$ in the GROUP (0="none"; $1=$ "less than weekly"; 2="weekly"; 3 ="daily"). Consistent with previous work, ${ }^{9,29-31}$ a binary regular cannabis use variable was constructed by using the cut-off value of once or more per week during the lifetime period of most frequent use.

In accordance with previous studies investigating the association between season of birth and schizophrenia in the Northern hemisphere sites, ${ }^{32}$ the high-risk birth period was defined based on the winter solstice (December-March), and a binary winter-birth exposure was constructed. Hearing impairment was defined based on self-reported hearing impairment in the last 12 months ( $0=$ "absent" and $1=$ "present").

The history of bullying by peers (emotional, psychological or physical violence) before 17 years of age was assessed using the short version of the Retrospective Bullying Questionnaire $(\mathrm{RBQ}),{ }^{33,34}$ that measures the severity of the bullying experience: $0=$ "none"; $1=$ "some (no physical injuries)"; 2="moderate (minor injuries or transient emotional reactions)"; 
3="marked (severe and frequent physical or psychological harm)". Exposure to childhood bullying was dichotomized using $\geq 1$ as the cut-off point ( $0=$ "absent" and $\geq 1$ ="present").

\section{Polygenic risk score for schizophrenia}

Samples of all individuals were genotyped at Cardiff University Institute of Psychological Medicine and Clinical Neurology, using custom Illumina HumanCoreExome-24 BeadChip genotyping arrays containing probes for 570038 genetic variants (Illumina, San Diego, CA). Genotype data were called using the GenomeStudio package and transferred into PLINK format for further analysis. Quality control was conducted in PLINK v1.07 $7^{35}$ or with custom Perl scripts. Variants with call rate $<98 \%$ were excluded from the dataset. Hardy-Weinberg Equilibrium p value was calculated separately in Turkish, northern European and southern European samples. Variants with Hardy-Weinberg Equilibrium p value $<1 \times 10-6$ in any of these three regions were excluded from the dataset. After QC, 559505 variants remained. Samples with call rate $<98 \%$ were excluded from the dataset. A linkage disequilibrium pruned set of variants was calculated using the - indep-pairwise command in PLINK (maximum r2 $=0.25$, window size $=500$ SNPs, window step size $=50$ SNPs) and used for further analyses. Homozygosity $\mathrm{F}$ values were calculated using the - het command in PLINK, and outlier samples $(\mathrm{F}<-0.11$ or $\mathrm{F}>0.15)$ were excluded. The genotypic sex of samples was calculated from X chromosome data using the - check-sex command in PLINK, and samples with different genotypic sex to their database sex were excluded. Identity-by-descent (IBD) values were calculated for the sample in PLINK. Samples with one or more siblings among the genotyped samples according to the database but no identified genotypic siblings (defined as PI-HAT $>0.35$ and $<0.65$ ) were excluded. After these were removed from consideration, samples with two or more siblings in the database that were not supported by the genotypic data were also excluded. After visually observing clustering of errors by genotyping chip, we decided to exclude chips with a high proportion of errors. All samples on chips with five or more sample exclusions due to heterozygosity or call rate (out of 12 possible samples) were excluded. All samples on chips with four or more sample exclusions due to sex or relative checks were also excluded unless their identity was corroborated by concordance between database and genotype relatedness data with a sample on another chip.

Principal components (PCs) were calculated in PLINK using LD pruned variants after combining the dataset with the Thousand Genomes reference dataset. Due to the inherently multi-population nature of the dataset and the variety of possible analyses, no exclusions were made to the whole dataset based on this analysis; population effects were corrected for separately in individual analyses. 
After quality control, genotypes were imputed on the Michigan Imputation Server using the Haplotype Reference Consortium reference panel (version 1.1) and the programs Eagle for haplotype phasing and Minimac3 for imputation. ${ }^{36,37}$ After imputation, variants with an imputation $\mathrm{r} 2>0.6, \mathrm{MAF}>0.1 \%$ and call rate $>99 \%$ were retained (8 277535 variants). Best-guess genotypes were generated from genotype probabilities using PLINK.

PRS-SCZ was constructed using summary statistics from the PGC2 genome-wide association study in both samples. ${ }^{5}$ Clumping was performed in imputed best-guess genotypes for each dataset using PLINK (maximum $\mathrm{r} 2=0.2$, window size $=500 \mathrm{~kb}$, minimum MAF $=10 \%$, minimum INFO score $=0.7$ ), and variants within regions of long-range $\mathrm{LD}$ around the genome (including the MHC) excluded. ${ }^{38} \mathrm{PRS}-\mathrm{SCZ}$ were then constructed from best-guess genotypes using PLINK at 10 different p-value thresholds $(\mathrm{PT}=1,0.5,0.3,0.2,0.1,0.05$, $0.01,1 \times 10-4,1 \times 10-6,5 \times 10-8)$. Consistent with previous research in the field ${ }^{39,40}$ and previous work in this dataset, we used $\mathrm{PT}=0.05$ for our primary analysis, as this threshold explained the most variation in the phenotype in the PGC2 analysis. ${ }^{5}$

\section{Statistical Analysis}

Stata software version 15.0 was used for the analysis. ${ }^{41}$ Supplementary Table S1 reports missing data. The analyses were conducted on both multiple imputed data and raw data. Under the assumption of missing at random, the multiple imputation chained equation ${ }^{42}$ was applied with 20 imputations restricted to in-range values (relative efficiency $\geq 99 \%$ ). ESSCZ was calculated after imputing missing values of the environmental exposures (cannabis use, hearing impairment, winter-birth, and childhood adversity domains). All the analyses were run on multiple imputed data and pooled using Rubin's rules. ${ }^{43}$ To test geneenvironment interaction, additive models were chosen over multiplicative models prior to data collection (EUGEI consortium meeting, December 14, 2013), consistent with previous work. ${ }^{9}$ For all analyses, random intercept multilevel mixed regression models, taking into account clustering of participants within countries, were applied. Models including PRSSCZ were a priori adjusted for ancestry using 10 PCs and adjusted models included age and sex as covariates. The nominal significance threshold was set to $P=0.05$.

For the case-control analyses, consistent with previous analyses, ${ }^{9}$ ES-SCZ and PRS-SCZ were dichotomized at the quartile cut-off points based on the control distribution within each country (to account for differences between countries that may arise due to ethnic and geographical variation). The highest quartile was considered the binary risk state for schizophrenia (hereafter: PRS-SCZ 75 and ES-SCZ 75 ). Multilevel logistic regression models were applied to test the independent and joint effects of PRS-SCZ 75 and ES-SCZ 75 
(independent variables) on the diagnosis of schizophrenia (i.e. case-control status; dependent variable). Departure from additivity was tested using the relative excess risk due to interaction (RERI). ${ }^{44,45}$ RERI greater than zero was defined as a positive deviation from additivity, and considered significant when the $95 \%$ CI did not contain zero. Conforming to early work in this sample, we applied the delta method to calculate the RERI using the ORs derived from the model. ${ }^{?}$

In unaffected siblings and healthy comparison participants, the effects of continuous measures of PRS-SCZ, ES-SCZ, and their interaction on continuous measures of schizotypy dimensions (total, positive, and negative; dependent variables) were tested with multilevel linear regression models, where the coefficient of the product term (PRS-SCZ*ES-SCZ) reflects the departure from additivity. ${ }^{46}$

\section{Results}

Sample demographic data, SIS-R scores, PRS-SCZ 75 and ES-SCZ 75 distributions are reported in Table 1. Missing data are reported in the Supplementary material (Table S1). PRS-SCZ explained $15 \%$ of the variance in case-control status (OR $=1.30$ [95\% CI: $1.25-1.34$ ] $P$ $<0.001)$ and $20 \%$ after adjusting for age, sex and country (OR $=1.30$ [95\% CI: 1.26, 1.35], $P<0.001)$. ES-SCZ explained $28 \%$ of the variance in case-control status $(\mathrm{OR}=2.52[95 \%$ CI: $2.29-2.78], P<0.001)$ and $33 \%$ after adjusting for age, sex, and country $(\mathrm{OR}=2.40$ [95\% CI: 2.17, 2.66], $P<0.001)$. There was no evidence for gene-environment correlation, as PRS-SCZ 75 was not strongly or significantly associated with ES-SCZ 75 in the control group $(\mathrm{OR}=1.08$ [95\% CI: $0.78,1.51], P=0.635)$, neither after adjusting for age and sex $(\mathrm{OR}=$ 1.08 [95\% CI: $0.78,1.51], P=0.638)$.

Table 1 Sample characteristics

\begin{tabular}{llcccc}
\hline & & $\begin{array}{c}\text { Patients } \\
\mathrm{n}=1699\end{array}$ & $\begin{array}{c}\text { Siblings } \\
\mathrm{n}=1753\end{array}$ & $\begin{array}{c}\text { Controls } \\
\mathrm{n}=1542\end{array}$ & $\begin{array}{c}\text { Total } \\
\mathrm{N}=4994\end{array}$ \\
\hline Age & Mean (S.D.) & $31.49(8.95)$ & $31.73(9.62)$ & $33.45(10.62)$ & $32.18(9.76)$ \\
Sex & \% female & 30.02 & 53.17 & 50.58 & 44.49 \\
SIS-R total & Mean (S.D.) & - & $0.39(0.33)$ & $0.23(0.24)$ & $0.32(0.30)^{\mathrm{a}}$ \\
SIS-R positive & Mean (S.D.) & - & $0.41(0.42)$ & $0.24(0.31)$ & $0.33(0.38)^{\mathrm{a}}$ \\
SIS-R negative & Mean (S.D.) & - & $0.38(0.34)$ & $0.23(0.24)$ & $0.31(0.31)^{\mathrm{a}}$ \\
PRS-SCZ75 & $\%>75^{\text {th }}$ & 45.44 & 33.09 & 23.74 & 34.40 \\
ES-SCZ75 & $\%>75^{\text {th }}$ & 58.79 & 36.30 & 21.88 & 37.64 \\
\hline
\end{tabular}

${ }^{2}$ within siblings and controls groups (3295 individuals); ES-SCZ75: exposome score for schizophrenia (75\% cut-point); N: number of individuals; PRS-SCZ 75 : polygenic risk score for schizophrenia (75\% cut-point); S.D.: standard deviation; SIS-R: The Structured Interview for Schizotypy - Revised. 


\section{Main and joint effects of PRS-SCZ 75 and ES-SCZ 75 on case-control status}

PRS-SCZ 75 was associated with case status $(\mathrm{OR}=2.91$ [95\% CI: 2.48, 3.40], $P<0.001$; adjusted for age and sex: $\mathrm{OR}=2.85$ [95\% CI: 2.43, 3.35], $P<0.001)$; and ES-SCZ 75 was associated with case status $(\mathrm{OR}=4.99$ [95\% CI: 4.22, 5.90], $P<0.001$, adjusted for age and sex: $\mathrm{OR}=4.90$ [95\% CI: 4.14, 5.81], $P<0.001)$. There was evidence for a positive additive interaction between PRS-SCZ 75 and ES-SCZ 75 (RERI $=7.29$ [95\% CI: 3.73, 10.85], $P$ $<0.001$ ), also after adjusting for age and sex (Table 2 and Figure 1). Results from the analyses using unimputed data corroborated these results and are reported in the Supplementary material (Table S2 and Figure S3).

Table 2 Interaction of PRS-SCZ 75 and ES-SCZ 75 on case-control status

\begin{tabular}{lccc}
\hline & PRS-SCZ & \\
& Odds ratio $(95 \% \mathrm{CI})$ & Odds ratio $(95 \% \mathrm{CI})$ & RERI $(95 \% \mathrm{CI})$ \\
$\mathrm{ES}-\mathrm{SCZ}_{75}=0$ & 1.0 & $2.79(2.24-3.47)$ & \\
& & $P<0.001$ & \\
$\mathrm{ES}-\mathrm{SCZ}_{75}=1$ & $4.86(3.92-6.02)$ & $13.44(10.21-17.69)$ & $6.79(3.32-10.26)$ \\
& $P<0.001$ & $P<0.001$ & $P<0.001$ \\
\hline
\end{tabular}

Adjusted for sex, age and ten principal components; CI: confidence interval; ES-SCZ 75 : exposome score for schizophrenia (75\% cut-point); PRS-SCZ 75 : polygenic risk score for schizophrenia (75\% cut-point); RERI: relative excess risk due to interaction.

\section{Main and joint effects of continuous PRS-SCZ and ES-SCZ on SIS-R dimensions}

PRS-SCZ was significantly associated with the SIS-R dimensions in the unaffected sibling / healthy comparison participants sample (total: $\mathrm{B}=0.011$ [95\%CI: 0.006, 0.015], $P<0.001$; positive: $\mathrm{B}=0.012$ [95\%CI: 0.007, 0.018], $P<0.001$; negative: $\mathrm{B}=0.010$ [95\%CI: 0.005 , $0.014], P<0.001$ ) also after adjusting for age and sex (Table 3). ES-SCZ was also significantly associated with the SIS-R dimensions (total: $\mathrm{B}=0.088$ [95\%CI: 0.078, 0.098], $P<0.001$; positive: $\mathrm{B}=0.103$ [95\%CI: $0.090,0.116$ ] $P<0.001$; negative: $\mathrm{B}=0.074$ [95\%CI: 0.064 , 0.085 ], $P<0.001$ ), also after adjusting for age and sex (Table 3 ).

There was evidence for a significant interaction between ES-SCZ and PRS-SCZ on the SISR dimensions (total: $\mathrm{B}=0.006$ [95\% CI: 0.003, 0.009], $P<0.001$ ); positive: $\mathrm{B}=0.005$ [95\% CI: 0.002, 0.009], $P=0.002$ ); and negative: $\mathrm{B}=0.006$ [95\% CI: $0.003-0.009], P<0.001$ ), also after adjusting for age and sex (Table 3). Results from the analyses in unimputed data confirmed the results in imputed data and are reported in the Supplementary material (Table S4). 
2

0

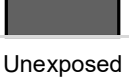

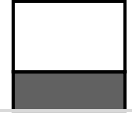

PRS-SCZ75

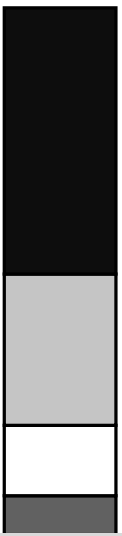

Both

口Unexposed aPRS-SCZ75 aES-SCZ75 घInteraction

Figure 1 Additive effect of the polygenic risk score for schizophrenia, 75\% cut-point (PRS-SCZ 75 ), and the exposome score for schizophrenia, 75\% cut-point (ES-SCZ 75 ) on case-control status, adjusted for age, sex and ten principal components; RERI: relative excess risk due to interaction

Table 3 Main and joint effects of PRS-SCZ and ES-SCZ on SIS-R scores.

\begin{tabular}{|c|c|c|c|c|c|c|c|c|c|}
\hline \multirow[b]{2}{*}{$\begin{array}{l}\text { Psychopathology } \\
\text { measures }\end{array}$} & \multicolumn{3}{|c|}{ Main effect PRS-SCZ a } & \multicolumn{3}{|c|}{ Main effect ES-SCZ } & \multicolumn{3}{|c|}{ Interaction ${ }^{a}$} \\
\hline & B & $95 \% \mathrm{CI}$ & $P$-value & B & $95 \% \mathrm{CI}$ & P-value & B & $95 \% \mathrm{CI}$ & $\begin{array}{c}P_{-} \\
\text {value }\end{array}$ \\
\hline & & 0.007 - & & & $0.078-$ & & & $0.003-$ & \\
\hline SIS-R total & 0.011 & 0.015 & $<0.001$ & 0.088 & 0.098 & $<0.001$ & 0.006 & 0.009 & $<0.001$ \\
\hline SIS-R positive & 0.012 & $\begin{array}{c}0.007- \\
0.018\end{array}$ & $<0.001$ & 0.103 & $\begin{array}{c}0.091- \\
0.116\end{array}$ & $<0.001$ & 0.006 & $\begin{array}{c}0.002- \\
0.009\end{array}$ & 0.002 \\
\hline SIS-R negative & 0.010 & $\begin{array}{c}0.005- \\
0.014\end{array}$ & $<0.001$ & 0.074 & $\begin{array}{c}0.064- \\
0.085\end{array}$ & $<0.001$ & 0.006 & $\begin{array}{c}0.004- \\
0.009\end{array}$ & $<0.001$ \\
\hline
\end{tabular}

All analyses were adjusted for age and sex, ${ }^{2}$ additionally adjusted for ten principal components; B: regression coefficient from the multilevel model; CI: confidence interval; ES-SCZ: exposome score for schizophrenia; PRS-SCZ: polygenic risk score for schizophrenia; SIS-R: The Structured Interview for Schizotypy - Revised. 


\section{Discussion}

To the best of our knowledge, this is the first study testing the role of gene-environment interaction using aggregate scores of environmental and genetic liability across the spectrum of psychosis expression. In the case-control design, we found evidence for additive interaction between PRS-SCZ and ES-SCZ increasing the odds for schizophrenia. Similarly, evidence emerged for an interaction between PRS-SCZ and ES-SCZ on schizotypal traits when investigating $\mathrm{GxE}$ interaction in the group of non-ill siblings and healthy comparison participants.

By using aggregate scores for genetic and environmental liability for schizophrenia, we provided further support for the role of gene-environment interaction in schizophrenia ${ }^{9}$ and replicated recent findings of suggestive, but not nominally statistically significant, additive interaction between PRS-SCZ and environmental risk score for schizophrenia in a first episode psychosis cohort. ${ }^{47}$ When PRS-SCZ 75 and $\mathrm{ES}_{-} \mathrm{SCZ}_{75}$ were analyzed as binary modes of risk factors, the RERI was 6.79 and the corresponding $95 \%$ CI was above 2, suggesting a "mechanistic" interaction, which means that the risk of developing schizophrenia for some individuals exists only when both genetic and environmental risks are present together but not when either genetic or environmental risk is present alone.

In a previous study, we demonstrated that the extent of subthreshold phenotypic expression of schizophrenia polygenic risk is contingent on having a sibling with psychotic disorder, suggesting a gene-environment interaction underlying schizotypy expression. ${ }^{21}$ In the light of this new evidence, we tested for the first time the putative role of gene-environment interaction in schizotypy traits. In line with our previous inference, we have now demonstrated that molecular genetic liability for schizophrenia moderates the effect of environmental liability for schizophrenia on phenotypic expression of overall, positive, and negative schizotypy traits in non-ill participants. Although much research has investigated the role of familial sensitivity to individual environmental exposures (e.g. cannabis use and childhood adversities) underlying subclinical psychosis expression, ${ }^{3,48}$ only few studies have utilized PRS to investigate the role of GxE in intermediate psychotic phenotypes. ${ }^{49}$ A recent study from the 1966 Northern Finland Birth Cohort showed that high birth weight, a risk factor for familial schizophrenia in this cohort, increased the association between PRS-SCZ and social anhedonia, suggesting gene-environment interaction. ${ }^{50}$ Similarly, a study conducted in a general population twin cohort demonstrated that while PRS-SCZ was not independently associated with affective dysregulation and psychosis proneness, PRS-SCZ increased sensitivity to the effect of childhood adversities on affective dysregulation and psychosis proneness. ${ }^{51}$ Although not a direct test of gene-environment interaction, a study of 
healthy young males assessed during their compulsory military service showed that there was a negative association between PRS-SCZ and positive schizotypy at military induction (stressful condition) but not at follow-up, providing further support for the key role of environment in the phenotypic expression of schizotypy traits. ${ }^{52}$ Taken together, although warranting further replication in independent cohorts, these findings imply that the phenotypic expression of schizotypical traits involve underlying genomic liability for schizophrenia that operates, at least in part, through sensitizing individuals to the exposome.

The major strengths related to the study population were twofold: sufficient sample size to detect gene-environment interactions and access to comprehensive genotype, phenotype, and exposure data collected through validated interviews conducted by trained psychiatrists, psychologists or research assistants. Further, ES-SCZ was constructed using predictive modelling that mutually adjusted for the interdependency of exposures to prevent overestimation of the weights per exposure. ES-SCZ was fully compatible with this study population and clearly outperformed other aggregate scores that were based on metaanalytical estimates or simple summation of exposures as shown previously. Notwithstanding, ES-SCZ was limited by the degree to which exposures were available in the dataset, and therefore did not include other exposures that might be of importance, such as obstetric and pregnancy complications. The cross-sectional design did not allow for investigating the dynamic nature of gene-environment interaction over time.

In conclusion, we have shown that the interplay between exposome load and genetic liability for schizophrenia contributes to phenotypical expression of psychosis across the extended phenotype. Our findings provide further empirical support to the notion of etiological continuity of psychosis spectrum and paves the way for future longitudinal studies of schizotypy traits in the general population. 


\section{Appendix}

Genetic Risk and Outcome of Psychosis (GROUP) Investigators in EUGEI (GROUPEUGEI) investigators are: Behrooz Z. Alizadeh ${ }^{\mathrm{a}}$, Therese van Amelsvoort ${ }^{\mathrm{b}}$, Richard Bruggeman $^{\mathrm{a}}$, Wiepke Cahn ${ }^{\mathrm{c,d}}$, Lieuwe de Haan $^{\mathrm{e}}$, Bart P. F. Rutten ${ }^{\mathrm{b}}$, Jurjen J. Luykx ${ }^{\mathrm{c}, \mathrm{fg},}$, Jim van Os ${ }^{\mathrm{c}, \mathrm{b}, \mathrm{h}}$ and Ruud van Winkel ${ }^{\mathrm{b}, \mathrm{i}}$

${ }^{a}$ University of Groningen, University Medical Center Groningen, University Center for Psychiatry, Rob Giel Research Center, Groningen, The Netherlands

${ }^{b}$ Maastricht University Medical Center, Department of Psychiatry and Neuropsychology, School for Mental Health and Neuroscience, Maastricht, The Netherlands

c University Medical Center Utrecht, Department of Psychiatry, UMC Utrecht Brain Centre, Utrecht University, Utrecht, The Netherlands

${ }^{d}$ Altrecht, General Menthal Health Care, Utrecht, The Netherlands

e Amsterdam UMC, University of Amsterdam, Department of Psychiatry, Amsterdam, The Netherlands

${ }^{f}$ GGNet Mental Health, Apeldoorn, The Netherlands

g Department of Translational Neuroscience, UMC Utrecht Brain Center, University Medical Center Utrecht, Utrecht University, Utrecht, The Netherlands

${ }^{\mathrm{h}}$ King's College London, King's Health Partners, Department of Psychosis Studies, Institute of Psychiatry, London, UK

${ }^{i}$ KU Leuven, Department of Neuroscience, Research Group Psychiatry, Leuven, Belgium

\section{Acknowledgements}

The authors are grateful to the patients and their families for participating in the project. They also thank all research personnel involved in the GROUP project, in particular J. van Baaren, E. Veermans, G. Driessen, T. Driesen, E. van't Hag and J. de Nijs. All the DNA samples from Turkey were provided by the Ankara University Brain Research Center Biobank, that was supported by Ankara University Scientic Research Projects Coordination Unit (project no. 10A6055003, 2010).

\section{Financial Support}

The EUGEI project was supported by by the European Community's Seventh Framework Program under grant agreement No. HEALTH-F2-2009-241909 (Project EU-GEI). Dr O'Donovan is supported by MRC programme grant (G08005009) and an MRC Centre grant (MR/L010305/1). Dr Rutten was funded by a VIDI award number 91718336 from the Netherlands Scientific Organisation. Drs Guloksuz and van Os are supported by 
the Ophelia research project, ZonMw grant number: 636340001. Dr Arango was supported by the Spanish Ministry of Science and Innovation. Instituto de Salud Carlos III (SAM16PE07CP1, PI16/02012, PI19/024), CIBERSAM. Madrid Regional Government (B2017/BMD-3740 AGES-CM-2), Fundación Familia Alonso and Fundación Alicia Koplowitz.

\section{Conflicts of Interest}

Celso Arango. has been a consultant to or has received honoraria or grants from Acadia, Angelini, Gedeon Richter, Janssen Cilag, Lundbeck, Minerva, Otsuka, Roche, Sage, Servier, Shire, Schering Plough, Sumitomo Dainippon Pharma, Sunovion and Takeda. Michael O'Donovan is supported by a collaborative research grant from Takeda Pharmaceuticals. 


\section{References}

1. Guloksuz S, van Os J. The slow death of the concept of schizophrenia and the painful birth of the psychosis spectrum. Psychol Med. 2018;48(2):229-244

2. Van Os J, Linscott RJ, Myin-Germeys I, Delespaul P, Krabbendam L. A systematic review and metaanalysis of the psychosis continuum: evidence for a psychosis proneness-persistence-impairment model of psychotic disorder. Psychol Med. 2009;39(2):179-195.

3. European Network of National Networks studying Gene-Environment Interactions in Schizophrenia (EU-GEI), van Os J, Rutten BP, et al. Identifying gene-environment interactions in schizophrenia: contemporary challenges for integrated, large-scale investigations. Schizophr Bull. 2014;40(4):729736.

4. Hilker R, Helenius D, Fagerlund B, et al. Heritability of Schizophrenia and Schizophrenia Spectrum Based on the Nationwide Danish Twin Register. Biol Psychiatry. 2018;83(6):492-498.

5. Schizophrenia Working Group of the Psychiatric Genomics C. Biological insights from 108 schizophrenia-associated genetic loci. Nature. 2014;511(7510):421-427.

6. Pardinas AF, Holmans P, Pocklington AJ, et al. Common schizophrenia alleles are enriched in mutation-intolerant genes and in regions under strong background selection. Nat Genet. 2018;50(3):381-389.

7. Radua J, Ramella-Cravaro V, Ioannidis JPA, et al. What causes psychosis? An umbrella review of risk and protective factors. World Psychiatry. 2018;17(1):49-66.

8. Stilo SA, Murray RM. Non-Genetic Factors in Schizophrenia. Curr Psychiatry Rep. 2019;21(10):100.

9. Guloksuz S, Pries LK, Delespaul P, et al. Examining the independent and joint effects of molecular genetic liability and environmental exposures in schizophrenia: results from the EUGEI study. World Psychiatry. 2019;18(2):173-182.

10. Guloksuz S, Rutten BPF, Pries LK, et al. The Complexities of Evaluating the Exposome in Psychiatry: A Data-Driven Illustration of Challenges and Some Propositions for Amendments. Schizophr Bull. 2018;44(6):11751179.

11. Pries L-K, Lage-Castellanos A, Delespaul P, et al. Estimating Exposome Score for Schizophrenia Using Predictive Modeling Approach in Two Independent Samples: The Results From the
EUGEI Study. Schizophr Bull. 2019;45(5):960965.

12. Guloksuz S, Pries LK, Ten Have M, et al. Association of preceding psychosis risk states and non-psychotic mental disorders with incidence of clinical psychosis in the general population: a prospective study in the NEMESIS-2 cohort. World Psychiatry. 2020;19(2):199-205.

13. Pries L-K, van Os J, ten Have M, et al. Association of recent stressful life events with mental and physical health in the context of genomic and exposomic liability for schizophrenia. JAMA psychiatry. accepted.

14. Korver N, Quee PJ, Boos HB, Simons CJ, de Haan L, GROUP investigators. Genetic Risk and Outcome of Psychosis (GROUP), a multi-site longitudinal cohort study focused on geneenvironment interaction: objectives, sample characteristics, recruitment and assessment methods. Int J Methods Psychiatr Res. 2012;21(3):205-221.

15. Van Os J, Rutten BP, Myin-Germeys I, et al. Identifying gene-environment interactions in schizophrenia: Contemporary challenges for integrated, large-scale investigations. Schizophr Bull. 2014;40(4):729-736.

16. McGuffin P, Farmer A, Harvey I. A polydiagnostic application of operational criteria in studies of psychotic illness. Development and reliability of the OPCRIT system. Arch Gen Psychiatry. 1991;48(8):764-770.

17. Wing JK, Babor T, Brugha T, et al. SCAN. Schedules for Clinical Assessment in Neuropsychiatry. Arch Gen Psychiatry. 1990;47(6):589-593.

18. Andreasen NC, Flaum M, Arndt S. The Comprehensive Assessment of Symptoms and History (CASH). An instrument for assessing diagnosis and psychopathology. Arch Gen Psychiatry. 1992;49(8):615-623

19. Kendler KS, Lieberman JA, Walsh D. The Structured Interview for Schizotypy (SIS): a preliminary report. Schizophr Bull. 1989;15(4):559-571.

20. Vollema MG, Ormel J. The reliability of the structured interview for schizotypy-revised. Schizophr Bull. 2000;26(3):619-629.

21. van Os J, Pries LK, Delespaul P, et al. Replicated evidence that endophenotypic expression of schizophrenia polygenic risk is greater in healthy siblings of patients compared to controls, suggesting 
gene-environment interaction. The EUGEI study. Psychol Med. 2019:1-14.

22. Pries LK, Guloksuz S, Menne-Lothmann C, et al. White noise speech illusion and psychosis expression: An experimental investigation of psychosis liability. PloS one. 2017;12(8):e0183695.

23. Bernstein DP, Stein JA, Newcomb MD, et al. Development and validation of a brief screening version of the Childhood Trauma Questionnaire. Child Abuse Negl. 2003;27(2):169-190.

24. Sar V, Akyuz G, Kundakci T, Kiziltan E, Dogan O. Childhood trauma, dissociation, and psychiatric comorbidity in patients with conversion disorder. The American journal of psychiatry. 2004;161(12):2271-2276.

25. Thombs BD, Bernstein DP, Lobbestael J, Arntz A. A validation study of the Dutch Childhood Trauma Questionnaire-Short Form: factor structure, reliability, and known-groups validity. Child Abuse Negl. 2009;33(8):518-523.

26. Hernandez A, Gallardo-Pujol D, Pereda N, et al. Initial validation of the Spanish childhood trauma questionnaire-short form: factor structure, reliability and association with parenting. $J$ Interpers Violence. 2013;28(7):1498-1518.

27. Barkus EJ, Stirling J, Hopkins RS, Lewis S. Cannabis-induced psychosis-like experiences are associated with high schizotypy. Psychopathology. 2006;39(4):175-178.

28. Robins LN, Wing J, Ulrich Wittchen $\mathrm{H}$, et al. The composite international diagnostic interview. An epidemiologic instrument suitable for use in conjunction with different diagnostic systems and in different cultures. Annual Review of Addictions Research and Treatment. 1991;1(C):263-271.

29. Van Winkel R, Van Beveren NJ, Simons C, Outcome of Psychosis Investigators. AKT1 moderation of cannabis-induced cognitive alterations in psychotic disorder. Neuropsychopharmacology. 2011;36(12):25292537.

30. Pries LK, Guloksuz S, Ten Have M, et al. Evidence That Environmental and Familial Risks for Psychosis Additively Impact a Multidimensional Subthreshold Psychosis Syndrome. Schizophr Bull. 2018;44(4):710-719.

31. Radhakrishnan R, Guloksuz S, Ten Have M, et al. Interaction between environmental and familial affective risk impacts psychosis admixture in states of affective dysregulation. Psychol Med. 2019;49(11):1879-1889.

32. Davies G, Welham J, Chant D, Torrey EF, McGrath J. A systematic review and meta-analysis of Northern Hemisphere season of birth studies in schizophrenia. Schizophr Bull. 2003;29(3):587593.

33. Hunter SC, Mora-Merchan J, Ortega R. The longterm effects of coping strategy use in victims of bullying. Span J Psychol. 2004;7(1):3-12.

34. Schäfer M, Korn S, Smith PK, et al. Lonely in the crowd: Recollections of bullying. British Journal of Developmental Psychology. 2004;22(3):379-394.

35. Purcell S, Neale B, Todd-Brown K, et al. PLINK: a tool set for whole-genome association and population-based linkage analyses. Am J Hum Genet. 2007;81(3):559-575.

36. Bunney PE, Zink AN, Holm AA, Billington CJ, Kotz CM. Orexin activation counteracts decreases in nonexercise activity thermogenesis (NEAT) caused by high-fat diet. Physiol Behav. 2017;176(10):139-148.

37. Loh P, Danecek P, Palamara PF, et al. Consortium panel. 2017;48(11):1443-1448.

38. Price AL, Weale ME, Patterson N, et al. Longrange $L D$ can confound genome scans in admixed populations. Am J Hum Genet. 2008;83(1):132135; author reply 135-139.

39. Allardyce J, Leonenko G, Hamshere M, et al. Association Between Schizophrenia-Related Polygenic Liability and the Occurrence and Level of Mood-Incongruent Psychotic Symptoms in Bipolar Disorder. JAMA psychiatry. 2018;75(1):28-35.

40. Sorensen HJ, Debost JC, Agerbo E, et al. Polygenic Risk Scores, School Achievement, and Risk for Schizophrenia: A Danish Population-Based Study. Biol Psychiatry. 2018;84(9):684-691.

41. Stata Statistical Software: Release 15. College Station, TX: StataCorp LLC [computer program]. 2017.

42. Royston P, White IR. Multiple Imputation by Chained Equations (MICE): Implementation in Stata. Journal of Statistical Software. 2011;45(4).

43. Rubin DB. Multiple imputation for nonresponse in surveys. Vol 81: John Wiley \& Sons; 2004.

44. Knol MJ, VanderWeele TJ. Recommendations for presenting analyses of effect modification and interaction. Int J Epidemiol. 2012;41(2):514-520.

45. VanderWeele TJ, Knol MJ. A Tutorial on Interaction. Epidemiologic Methods. 2014;3(1):3372

46. Knol MJ, van der Tweel I, Grobbee DE, Numans ME, Geerlings MI. Estimating interaction on an additive scale between continuous determinants in a logistic regression model. Int J Epidemiol. 2007;36(5):1111-1118.

47. Mas S, Boloc D, Rodriguez N, et al. Examining Gene-Environment Interactions Using Aggregate 
Scores in a First-Episode Psychosis Cohort. Schizophr Bull. 2020.

48. Modinos G, Iyegbe C, Prata D, et al. Molecular genetic gene-environment studies using candidate genes in schizophrenia: a systematic review. Schizophr Res. 2013;150(2-3):356-365.

49. Ronald A, Pain O. A systematic review of genomewide research on psychotic experiences and negative symptom traits: new revelations and implications for psychiatry. Hum Mol Genet. 2018;27(R2):R136-R152.

50. Liuhanen J, Suvisaari J, Kajantie E, et al. Interaction between compound genetic risk for schizophrenia and high birth weight contributes to social anhedonia and schizophrenia in women. Psychiatry Res. 2018;259:148-153.

51. Pries LK, Klingenberg B, Menne-Lothmann C, et al. Polygenic liability for schizophrenia and childhood adversity influences daily-life emotion dysregulation and psychosis proneness. Acta Psychiatr Scand. 2020;141(5):465-475.

52. Hatzimanolis A, Avramopoulos D, Arking DE, et al. Stress-Dependent Association Between Polygenic Risk for Schizophrenia and Schizotypal Traits in Young Army Recruits. Schizophr Bull. 2018;44(2):338-347 


\section{Supplementary material}

Supplementary table S1 Missing data

\begin{tabular}{|c|c|c|c|c|}
\hline & $\begin{array}{c}\text { Cases } \\
\mathrm{n}=1699\end{array}$ & $\begin{array}{l}\text { Siblings } \\
\mathrm{n}=1753\end{array}$ & $\begin{array}{l}\text { Controls } \\
\mathrm{n}=1542\end{array}$ & $\begin{array}{c}\text { Total } \\
\mathrm{N}=4994\end{array}$ \\
\hline SIS-R total score & - & $88(5.0 \%)$ & $10(0.7 \%)$ & $98(3.0 \%)^{\mathrm{a}}$ \\
\hline SIS-R positive score & - & $35(2.0 \%)$ & $8(0.5 \%)$ & $43(1.3 \%)^{\mathrm{a}}$ \\
\hline SIS-R negative score & - & $96(5.5 \%)$ & $13(0.8 \%)$ & $109(3.3 \%)^{\mathrm{a}}$ \\
\hline Cannabis use & $158(9.3 \%)$ & $109(6.2 \%)$ & $44(2.9 \%)$ & $311(6.2 \%)$ \\
\hline Bullying & $321(18.9 \%)$ & $320(18.3 \%)$ & $226(14.7 \%)$ & $867(17.4 \%)$ \\
\hline Emotional abuse & $384(22.6 \%)$ & $268(15.3 \%)$ & $91(5.9 \%)$ & $743(14.9 \%)$ \\
\hline Physical abuse & $372(21.9 \%)$ & $248(14.2 \%)$ & $78(5.1 \%)$ & $698(14.0 \%)$ \\
\hline Sexual abuse & $367(21.6 \%)$ & $260(14.8 \%)$ & $89(5.8 \%)$ & $716(14.3 \%)$ \\
\hline Emotional neglect & $384(22.6 \%)$ & $278(15.9 \%)$ & $89(5.8 \%)$ & $751(15.0 \%)$ \\
\hline Physical neglect & $373(22.0 \%)$ & $273(15.6 \%)$ & $84(5.5 \%)$ & $730(14.6 \%)$ \\
\hline Winter birth & $62(3.7 \%)$ & $90(5.1 \%)$ & $32(2.1 \%)$ & $184(3.7 \%)$ \\
\hline Hearing impairment & $102(6.0 \%)$ & $44(2.5 \%)$ & $39(2.5 \%)$ & $185(3.7 \%)$ \\
\hline
\end{tabular}

${ }^{a}$ percentage within siblings and controls groups (3295 individuals); N: number of observations; SIS-R: The Structured Interview for Schizotypy - Revised.

Supplementary table $\mathbf{S 2}$ Interaction of PRS-SCZ 75 and $E S-S C Z_{75}$ on case-control status in unimputed data

\begin{tabular}{|c|c|c|c|c|c|}
\hline & \multicolumn{2}{|c|}{ PRS-SCZ $75=0$} & \multicolumn{2}{|c|}{ PRS-SCZ $75=1$} & \multirow[b]{2}{*}{ RERI $(95 \% \mathrm{CI})$} \\
\hline & $\mathrm{N}$ case/control & $\begin{array}{l}\text { Odds ratio } \\
(95 \% \mathrm{CI})\end{array}$ & $\mathrm{N}$ case/control & $\begin{array}{l}\text { Odds ratio } \\
(95 \% \mathrm{CI})\end{array}$ & \\
\hline $\mathrm{ES}-\mathrm{SCZ}_{75}=0$ & $216 / 680$ & 1.0 & $164 / 216$ & $\begin{array}{c}2.52(1.92-3.31) \\
P<0.001\end{array}$ & 6.64 \\
\hline $\mathrm{ES}-\mathrm{SCZ}_{75}=1$ & $309 / 188$ & $\begin{array}{c}5.33(4.14-6.87) \\
P<0.001\end{array}$ & $233 / 63$ & $\begin{array}{c}13.49(9.57-19.02) \\
P<0.001\end{array}$ & $\begin{array}{c}P=0.003 \\
P\end{array}$ \\
\hline
\end{tabular}

Adjusted for sex, age and ten principal components; CI: confidence interval; ES-SCZ75: exposome score for schizophrenia (75\% cut-point); PRS-SCZ 75 : polygenic risk score for schizophrenia (75\% cut-point); RERI: relative excess risk due to interaction. 


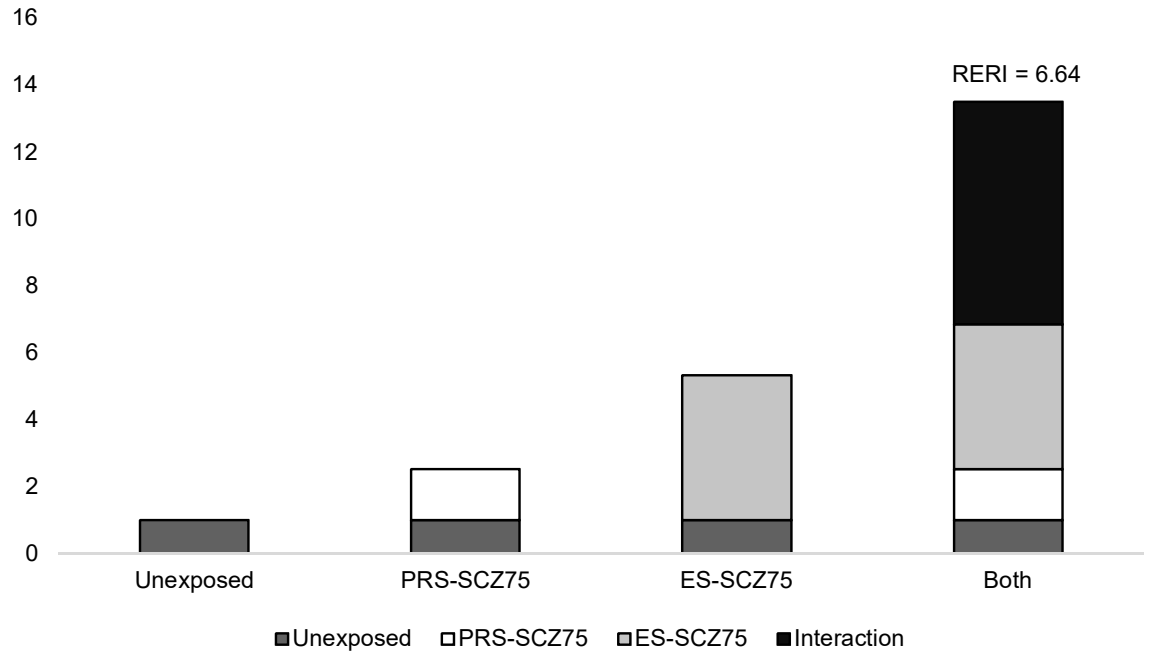

Additive effect of the polygenic risk score for schizophrenia, 75\% cut-point (PRS-SCZ), and the exposome score for schizophrenia, 75\% cut-point (ES-SCZ) on case-control status; adjusted for age, sex and ten principal components; RERI: relative excess risk due to interaction.

\section{Supplementary table S4 Main and joint effects of PRS-SCZ and ES-SCZ on SIS-R scores in unimputed data}

\begin{tabular}{|c|c|c|c|c|c|c|c|c|c|}
\hline \multirow{2}{*}{$\begin{array}{l}\text { Psychopathology } \\
\text { measures }\end{array}$} & \multicolumn{3}{|c|}{ Main effect PRS-SCZ } & \multicolumn{3}{|c|}{ Main effect ES-SCZ } & \multicolumn{3}{|c|}{ Interaction $^{\mathrm{b}}$} \\
\hline & B & $95 \% \mathrm{CI}$ & P-value & B & $95 \% \mathrm{CI}$ & P-value & B & $95 \% \mathrm{CI}$ & P-value \\
\hline \multicolumn{10}{|l|}{ Unadjusted } \\
\hline SIS-R total & 0.011 & $0.006-0.015$ & $<0.001$ & 0.091 & $0.079-0.102$ & $<0.001$ & 0.005 & $0.002-0.008$ & 0.001 \\
\hline SIS-R positive & 0.012 & $0.007-0.018$ & $<0.001$ & 0.101 & $0.087-0.106$ & $<0.001$ & 0.004 & $0.000-0.008$ & 0.027 \\
\hline SIS-R negatie & 0.009 & $0.005-0.013$ & $<0.001$ & 0.078 & $0.066-0.090$ & $<0.001$ & 0.006 & $0.003-0.009$ & $<0.001$ \\
\hline \multicolumn{10}{|l|}{ Adjusted ${ }^{h}$} \\
\hline SIS-R total & 0.011 & $0.006-0.015$ & $<0.001$ & 0.091 & $0.080-0.103$ & $<0.001$ & 0.005 & $0.002-0.008$ & 0.001 \\
\hline SIS-R positive & 0.012 & $0.007-0.018$ & $<0.001$ & 0.102 & $0.087-0.117$ & $<0.001$ & 0.004 & $0.001-0.008$ & 0.024 \\
\hline SIS-R negative & 0.009 & $0.005-0.014$ & $<0.001$ & 0.079 & $0.066-0.091$ & $<0.001$ & 0.006 & $0.003-0.009$ & $<0.001$ \\
\hline
\end{tabular}

${ }^{\mathrm{a} A d j u s t e d ~ f o r ~ a g e ~ a n d ~ s e x, ~}{ }^{\mathrm{b}}$ adjusted for ten principal components; B: regression coefficient from the multilevel model; CI: confidence interval; ES-SCZ: exposome score for schizophrenia; PRS-SCZ: polygenic risk score for schizophrenia; SIS-R: The Structured Interview for Schizotypy - Revised. 




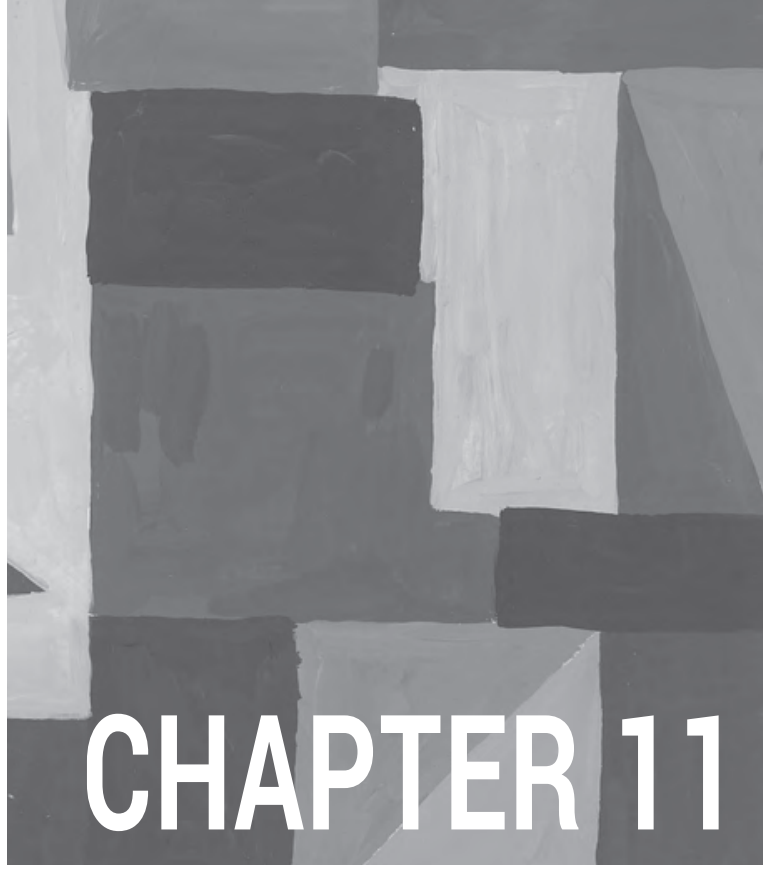

GENERAL DISCUSSION





The overall aim of this thesis was to examine large epidemiological datasets to understand processes and mechanisms that underlie the etiology of psychosis spectrum disorder (PSD). In the first part of this thesis, multidimensional psychopathology and related processes at population level were evaluated. In the second part of this thesis, the contribution of environmental and genetic factors on psychosis expression across the psychosis spectrum was tested. I developed and applied novel analytical tools to-for the first time-evaluate the exposomic and genomic liability network contributing to pluripotent psychopathology.

\section{How does subclinical psychosis expression relate to the development of psychosis spectrum?}

There is an active discussion on how subclinical psychopathology develops into clinical manifestation of psychosis phenotypes ${ }^{1-3}$. Although accumulating evidence suggests that positive psychosis expression is observed in nonpsychotic disorders ${ }^{1,3}$ and the general population $^{4,5}$, the literature draws attention to the restrictive concept of schizophrenia and positive psychosis expression as its most characteristic symptom ${ }^{1,3,6}$.

At-risk approaches build up on the prototypic concepts of psychotic disorders and rely greatly on attenuated positive psychosis expression ${ }^{1,7}$. However, studies evaluating the utility of the high-risk concept are conducted in risk-enriched samples and therefore provide limited insight into the utility of these concepts for preventive measures in the general population ${ }^{1}$. Therefore, chapter 2 conducts the first prospective epidemiological analyses of the clinicalhigh risk approach in the general population. The results demonstrate that the psychosis high-risk state was associated with a high relative risk to develop clinical psychosis outcome. However, due to its low prevalence in the general population, the population attributable fraction (PAF) shows that the fraction of clinical psychosis that could have been prevented if the high-risk state was successfully treated or prevention measures were set up, was relatively low. These results demonstrate the "prevention paradox" associated with early intervention of high-risk approaches.

The study also suggests that prevention approaches focusing on a broader multidimensional pathology, including especially psychosis expression, mood disorders and drug use disorders, might be a more effective strategy for population-based intervention than the restrictive focus on attenuated positive psychosis expression. Further, correlated symptom dimensions of PSD not only include positive psychotic symptoms (hallucinations and delusions) but also affective dysregulation (depression, mania), negative symptoms as well as motivational impairment (avolition or amotivation), and alterations in cognitive processing ${ }^{2}$. 
The results in chapter 3 support the conceptual shift to focus on multidimensional psychopathology and genetic as well as nongenetic factors. In line with converging evidence ${ }^{8-11}$, I demonstrate that several symptom dimensions (i.e. affective dysregulation, cognition alteration, negative symptoms), especially affective dysregulation, intensify the effect of environmental exposure and proxy genetic risk on positive psychosis expression. The study supports the notion of an affective pathway to psychosis phenotypes: under the influence of genetic and nongenetic factors, affective dysregulation may be complicated by aberrant salience attribution, which eventually may develop into clinical psychoses expression through the influence of the same genetic and nongenetic factors ${ }^{9,12}$.

To further explore underlying mechanisms and processes associated with intermediate phenotypes and multidimensional psychopathology, chapter 4 reviewed recent findings from the TwinssCan project. TwinssCan is a longitudinal general population twin cohort of young adults and adolescents, aiming to capture early psychopathological expression before the onset of clinical phenotypes. The review highlights important findings on the contribution of environmental and genetic factors on subclinical expression of psychosis and affective phenotypes as well as neurocognitive intermediate processes, such as salience attribution, reward sensitivity, and stress sensitivity. Further, the cohort provides tools to assess macro (e.g. monthly) as well as micro (e.g. momentary) level psychopathological changes.

Chapter 5 covers one of the studies conducted in the TwinssCan project, investigating possible neurocognitive mechanisms associated with positive psychosis expression. Previous studies suggest that salient attribution measured by the white noise task may cause speech illusions by increasing perceptual expectations in clinical samples ${ }^{13-15}$. However, findings in nonclinical samples remain inconclusive when assessing sensory processing by means of the white noise task ${ }^{13,14,16,17}$. These differences may be due to variations in methodology and sample characteristics. Therefore, the current study compared different methodological approaches ${ }^{13,14}$ in one general population cohort. The results do not suggest that a processing bias of sensory information during the white noise task is associated with trait expression of psychosis experiences (assessed with the Community Assessment of Psychic Experiences; CAPE and Structured Interview for Schizotypy-Revised; SIS-R) in the general population. Therefore, it supports the notion that mechanisms associated with psychosis expression may be different in clinical and general populations. 
Several aspects need to be considered that may distinguish between subgroups perceiving speech illusions. Some studies suggest that speech illusions with affective rather than neutral valence might be associated with psychosis expression ${ }^{17,18}$, although this was not indicated in the current study. Further, as above-mentioned, psychosis expression may be complicated by environmental and genetic vulnerability, as well as multidimensional pathology. Therefore, the intermediate phenotype assessed through the white noise paradigm might likewise be dependent on these factors. Testing this hypothesis, we recently applied the white noise task in an international sample with patients diagnosed with schizophrenia, unaffected siblings, and healthy controls ${ }^{19}$. The results indeed suggest that the association between speech illusions and group status (with different levels of proxy genetic risk) were dependent on trait expression of psychotic experiences (assessed with CAPE), cognitive alterations, and childhood adversity ${ }^{19}$.

In accordance with the literature, this thesis demonstrates that the presence of positive psychosis experiences in the general population may be associated with multidimensional psychopathology and in turn psychosis admixture may indicate severity of psychopathology $y^{9,11,20-25}$. While positive psychosis expression may indicate the potential risk to develop clinical psychopathology, several subgroups emerge $e^{4}$ : psychosis expression in $80 \%$ of individuals in the general population is transitory, only $20 \%$ experience persistent psychosis expression, and $7 \%$ of psychotic experiences at baseline may evolve into clinical psychosis phenotypes ${ }^{4}$. For the individuals who develop clinical syndromes, persistent psychosis expression may have been accompanied with environmental and genetic liability, which contributes to the expression of clinical psychopathology.

\section{Does the network of genetic and environmental factors contribute to the development of pluripotent psychopathology?}

There is increasing and active interest to understand the influence of the pleiotropic effects of genetic and nongenetic factors on psychopathology. The second part of the dissertation includes several articles on the joint and independent contribution of genetic and environmental exposure on the development of pluripotent psychopathology.

In accordance with previous studies ${ }^{26,27}$, chapter 7 indicates that polygenic risk for schizophrenia is associated with a diagnosis for schizophrenia. Estimates on the explained variance in this case-control sample were similar to previously reported estimates ${ }^{27,28}$. Furthermore, the study's results are in line with previous studies showing associations between the polygenic risk score for schizophrenia (PRS-SCZ) and disorder-related phenotypes: A recent longitudinal study following individuals with a first-admission 
psychosis over 20 years, showed that PRS-SCZ was associated with more severe negative symptoms, greater illness severity, and worse cognition at follow ups ${ }^{12}$. In another study PRSSCZ was linked to treatment response ${ }^{29}$.

However, an increasing number of studies also indicate low specificity of PRS-SCZ ${ }^{26,30}$ and transdiagnostic properties of genetic liability for schizophrenia ${ }^{28,31}$. PRS-SCZ is not specifically associated with a psychosis phenotype but is also commonly associated with the diagnosis of non-psychotic disorders ${ }^{26,28,30}$, medical conditions $s^{26,28}$, and low quality of life $e^{32}$.

To further understand the contribution of PRS-SCZ to PSD, investigating its effect on the early stages of psychopathology at population level is crucial. In the general population, the relationship between PRS-SCZ and symptom dimensions does not seem to follow a simple logic. According to the study presented in chapter 6 , there was no direct effect of PRS-SCZ on daily variations of psychosis expression and negative affect, while the results suggest a positive association between PRS-SCZ and positive affect. As this was the first study investigating PRS-SCZ and momentary variation of psychosis proneness and affective dysregulation using ecological momentary assessment (EMA), it is difficult to compare the results of this study to the literature on macro level psychopathology. However, studies commonly show inconsistent results on the association between PRS-SCZ and the expression of specific psychosis symptom dimensions $s^{28,33-41}$. Several studies could not detect an association between PRS-SCZ and psychosis expression ${ }^{34,37,38,40}$ and others even suggested a negative association between PRS-SCZ and schizotypy ${ }^{35,38}$.

PRS-SCZ estimates might be associated with pluripotent psychopathology ${ }^{28,34,35,42-44}$. This is in line with the study presented in chapter 9 indicating that PRS-SCZ in the general population is predictive of the development of nonspecific health problems, especially mental health. Thus, it is possible that in the general population, PRS-SCZ effects pluripotent psychopathology, and that other co-occurring factors may contribute to more severe outcomes and eventually the development of psychosis symptoms.

A significant part of pathoetiology may be explained by the influence of environment factors and gene-environment interaction. Stress-vulnerability models ${ }^{45}$ suggest that underlying genetic liability could make individuals more vulnerable to the effects of environmental exposures. In this regard, there was some evidence that PRS-SCZ interacts with environmental exposures (e.g. intra-uterine environmental exposure, exposure to early life cannabis use, or childhood adversity) on the development of schizophrenia ${ }^{46}$ and neuroimaging intermediate phenotypes such as cortical maturation ${ }^{47}$ or functional 
connectivity density ${ }^{48}$. However, a previous pilot study ${ }^{49}$ could not detect an interaction between childhood adversity and PRS-SCZ on the development of schizophrenia. With a sample size of 80 patients and 110 healthy controls, latter insignificant result may be due to low power.

Chapter 6 presents findings from the first systematic approach to test the interaction between PRS-SCZ and different environmental exposures (i.e. childhood adversities, cannabis use, winter birth, hearing impairment) on the development of schizophrenia in a large international case-control sample. The results suggest additive interactions between PRSSCZ and the presence of lifetime regular cannabis use as well as exposure to early-life adversities (sexual abuse, emotional abuse, emotional neglect, and bullying). A "mechanistic" interaction ${ }^{50}$ was indicated for emotional and sexual abuse, meaning some individuals may only develop a diagnosis of schizophrenia if both the genetic and nongenetic exposures are present.

These results were supported in chapter 10 which shows a "mechanistic" interaction between the exposome score for schizophrenia and PRS-SCZ on case-control status as well as schizotypy in healthy controls and unaffected siblings of patients. In line with this, an independent study also found an additive interaction between PRS-SCZ and aggregate environmental risk on the diagnosis of schizophrenia ${ }^{51}$. This line of research strongly supports the idea that the development of PSD is driven by a combination of environmental and genetic vulnerabilities.

In the general populations, initial studies investigating $\mathrm{GxE}$ on the development of psychosis phenotypes tested the interaction between candidate genes ${ }^{52,53}$ or proxy genetic risk $\mathrm{k}^{9,53-55}$ and environmental exposures. The study presented in chapter 3 shows that the effect of environmental and proxy genetic factors on psychosis expression is additive and indicate a dose-response relationship between the number of exposures and presence of psychosis expression. Furthermore, there is some support for stress-vulnerability models and GxE applying PRS for major depressive disorder on depression outcome ${ }^{56-58}$. However, so far, only a few studies tested GxE in the general population using the PRS-SCZ.

Two of these studies are presented in this thesis: The study presented in chapter 6 tests whether PRS-SCZ interacts with early and late stressors (childhood adversity and minor daily-life stressors) on mental processes in the largest ever EMA data collected in the general population. While PRS-SCZ does not interact with daily-life stressors, it interacts with childhood adversity on pluripotent psychopathology. This is in line with findings from 
chapter 9: PRS-SCZ does not interact with recent stressful life events on mental or physical health. In accordance with the neurodevelopmental hypothesis ${ }^{59,60}$, the results may suggest that especially early-life exposures in neurodevelopmentally sensitive periods contribute to the development of mental ill health. The findings help to understand the effects of the complex interactions between environmental and genetic factors on nonspecific health outcomes in the general population.

\section{Does the exposome score for schizophrenia affect pluripotent psychopathology?}

Converging evidence supports the notion that environmental factors such as obstetric complications, childhood adversity, peer-bullying, urban environment, proxy of social exclusion, cannabis use, and winter-birth contribute to the development of PSD and extended to this subclinical psychosis expression ${ }^{3,61,62}$. While studies suggest a dose-response relationship between exposures and psychopathological outcome (see chapter 3 and ${ }^{25,63,64}$ ), researchers commonly focus on one exposure at a time. These studies do not take into account of the dependent effect of individual exposures forming a network of interconnected environmental exposures, the exposome ${ }^{65,66}$.

The work in this thesis contributes greatly to the conceptualization and understanding of the exposome. In chapter $\mathbf{8}$, predictive modelling approach was applied to calculate and validate an exposome score for schizophrenia in two independent cohorts with individuals diagnosed with schizophrenia and healthy controls. The study shows that exposures are not independent and that a score taking into account of the dependent effect of various exposures is best equipped to assess environmental liability for schizophrenia. Further, in the independent test sample, the exposome score was able to distinguish between cases, unaffected siblings, and healthy controls.

In chapter 2, the exposome score was applied to validate psychosis-risk states in the general population; exposomic liability indicated increased risk for clinical psychosis. Furthermore, chapter 10 showed that the exposome score was associated with schizotypy in healthy controls and unaffected siblings of patients with schizophrenia and chapter 9 demonstrates that the exposome score for schizophrenia also pleiotropically associates with mental and physical health in the general population. These results are in concordance with studies indicating the nonspecific effect of environmental exposures commonly associated with PSD. 
In support of the stress-vulnerability model ${ }^{45}$, an increasing number of studies suggest that early environmental exposures may intensify the effects of stressful life events later in life ${ }^{67-74}$. This is also supported by the study presented in chapter 6 , which provided evidence that childhood adversity and daily-life stressors pleiotropically interact in driving emotional dysregulation and subtle psychosis expression in daily life. Furthermore, the prospective study in chapter 9 indicates that the interaction between the exosome score and stressful events later in life predicts mental as well as physical health in the general population. The findings demonstrate that early and late environmental exposures interact and contribute to the development of pluripotent psychopathology.

\section{Methodological considerations and future directions}

The thesis provides important findings on the early manifestation of pluripotent psychopathology. However, further studies in deeply phenotyped, independent prospective cohorts are essential. Intermediate phenotypes as well as genetic and environmental factors need to be assessed to study trajectories from subtle psychosis expression to the development of the clinical syndrome. In this thesis several experimental approaches are introduced to assess intermediate phenotypes and future studies using these tools may shed further light on mechanisms and intermediate phenotypes associated with pluripotent psychopathology.

To deepen our understanding on the effects of genetic and environmental liability, the findings in the current thesis need to be replicated. Despite numerous studies focusing on PRS-SCZ within clinical and non-clinical populations, results cannot yet be translated into clinical practice. So far, research was not able to identify polygenic risk as a biomarker for PSD and related disorders. It is possible that more complex models will have to be applied to explain the liability for clinical outcomes. To understand the biological processes associated with genetic mechanisms, studying the effect of specific biological pathways, also in interaction with corresponding environmental exposure (e.g. Childhood adversity $\mathrm{x}$ Glucocorticoid pathway), on the development of PSD is essential. Likewise, new integrative approaches assessing the complete network of genetic, epigenetic and exposomic factors will increase our knowledge base. While the current studies did not indicate a gene-environment correlation between different environmental exposures and PRS-SCZ, it is possible that polygenic risk may drive individuals to higher exposure of other environmental exposures such as urbanicity ${ }^{75}$. Therefore, for more complex models of genetic and environmental liability, including more environmental exposures, correlations between factors might need to be taken into account. 
The thesis contributes greatly to the understanding of the exposome. However, the inclusion of environmental exposures was restricted to available exposures within the used datasets. Extending the exposome could greatly contribute to its predictive power. In this regard, these studies indicate that environmental exposures might intensify each other's effect and that exposures at different time points (e.g. developmental sensitive times) may be weighted differently. Future research may take into account of interactions between exposures and temporal proximity.

Further, to fully understand mental ill health and pluripotent psychopathology, studies may not only focus on "risk" factors, but also factors promoting resilience. Studies previously indicated that psychological factors such as social support and having a purpose in life may promote resilience (see Appendix). Likewise, genetic and epigenetic factors can influence resilience against stress (see Appendix).

Future research on resilience may greatly benefit from methodological approaches presented in this thesis, that is the calculation of the exposome score for resilience and GxE focusing on resilience promoting markers. However, due to its dynamic and active nature, with individuals following different trajectories and interactively responding to environmental exposures, the study of resilience presents several methodological challenges (as indicated in the Appendix). Future research needs to take into account of the dynamic nature of resilience by prospectively investigating the response to well-defined stress exposure. Building up on research presented in this thesis, combining information on "risk" and "protective" factors will further increase our understanding of the development pluripotent psychopathology.

\section{Conclusion}

In conclusion, findings from the first part of the thesis give support to the notion that the development of psychosis phenotypes may be understood by the study of multidimensional psychopathology. Furthermore, studies on the early stages of psychopathology in the general population add to a better understanding of the underlying mechanisms contributing to psychosis expression along the spectrum. This includes concurrence of symptom dimensions as well as the driving force of genetic and environmental contributions to subclinical psychosis expression, affective dysregulation, negative symptoms and cognitive impairment.

The studies in the second part of the thesis contribute greatly to the understanding of genetic and environmental liability for clinical as well as subclinical psychosis phenotypes. The results support the notion that in the general population, genetic and environmental exposure may non-specifically affect pluripotent psychopathology. Furthermore, complication through 
psychosis admixture (especially with affective dysregulation) and the same genetic and environmental exposures may lead to the development of more severe outcomes and eventually psychosis syndrome. Analyses on $\mathrm{GxE}$ and the exposome for schizophrenia indicate that the whole network of environmental and genetic liability needs to be taken into account to understand psychosis phenotypes along the spectrum. 


\section{References}

1. van Os J, Guloksuz S. A critique of the "ultra-high risk" and "transition" paradigm. World Psychiatry. 2017;16(2):200-206.

2. van Os J, Kenis G, Rutten BP. The environment and schizophrenia. Nature. 2010;468(7321):203-212.

3. van Os J, Reininghaus U. Psychosis as a transdiagnostic and extended phenotype in the general population. World Psychiatry. 2016;15(2):118-124.

4. Linscott RJ, van Os J. An updated and conservative systematic review and metaanalysis of epidemiological evidence on psychotic experiences in children and adults: on the pathway from proneness to persistence to dimensional expression across mental disorders. Psychol Med. 2013;43(6):11331149.

5. Van Os J, Linscott RJ, Myin-Germeys I, Delespaul P, Krabbendam L. A systematic review and meta-analysis of the psychosis continuum: evidence for a psychosis proneness-persistence-impairment model of psychotic disorder. Psychological medicine. 2009;39(2):179-195.

6. van Os J. " Schizophrenia" does not exist. British Medical Journal. 2016;352.

7. R Yung A, Fusar-Poli P, Nelson B. The ultra high risk approach to define psychosis risk. Current pharmaceutical design. 2012;18(4):346-350.

8. Binbay T, Drukker M, Elbi H, et al. Testing the psychosis continuum: differential impact of genetic and nongenetic risk factors and comorbid psychopathology across the entire spectrum of psychosis. Schizophr Bull. 2012;38(5):992-1002.

9. Radhakrishnan R, Guloksuz S, Ten Have M, et al. Interaction between environmental and familial affective risk impacts psychosis admixture in states of affective dysregulation. Psychol Med. 2019;49(11):1879-1889.

10. Guloksuz S, van Nierop M, Bak M, et al. Exposure to environmental factors increases connectivity between symptom domains in the psychopathology network. $B M C$ psychiatry. 2016;16(1):223.

11. Guloksuz S, van Nierop M, Lieb R, van Winkel R, Wittchen H-U, van Os J. Evidence that the presence of psychosis in non-psychotic disorder is environmentdependent and mediated by severity of nonpsychotic psychopathology. Psychological medicine. 2015;45(11):2389-2401.

12. Jonas KG, Lencz T, Li K, et al. Schizophrenia polygenic risk score and 20 year course of illness in psychotic disorders. Transl Psychiatry. 2019;9(1):300.

13. Catalan A, Simons CJ, Bustamante S, et al. Novel evidence that attributing affectively salient signal to random noise is associated with psychosis. PloS one. 2014;9(7):e102520.

14. Galdos M, Simons C, Fernandez-Rivas A, et al. Affectively salient meaning in random noise: a task sensitive to psychosis liability. Schizophr Bull. 2011;37(6):1179-1186.

15. Vercammen A, de Haan EH, Aleman A. Hearing a voice in the noise: auditory hallucinations and speech perception. Psychol Med. 2008;38(8):1177-1184.

16. Randell J, Goyal M, Saunders J, Reed P. Effect of a context of concrete and abstract words on hallucinatory content in individuals scoring high in schizotypy. J Behav Ther Exp Psychiatry. 2011;42(2):149-153.

17. Rimvall MK, Clemmensen L, Munkholm A, et al. Introducing the White Noise task in childhood: associations between speech illusions and psychosis vulnerability. Psychol Med. 2016;46(13):2731-2740.

18. Daalman K, Boks MP, Diederen KM, et al. The same or different? A phenomenological comparison of auditory verbal hallucinations in healthy and psychotic individuals. J Clin Psychiatry. 2011;72(3):320-325.

19. Schepers E, Lousberg R, Guloksuz S, et al. White noise speech illusions: a traitdependent risk marker for psychotic disorder? 2019;10:676. 
20. Wigman JT, van Os J, Abidi L, et al. Subclinical psychotic experiences and bipolar spectrum features in depression: association with outcome of psychotherapy. Psychol Med. 2014;44(2):325-336.

21. Fusar-Poli P, Nelson B, Valmaggia L, Yung AR, McGuire PK. Comorbid depressive and anxiety disorders in 509 individuals with an at-risk mental state: impact on psychopathology and transition to psychosis. Schizophr Bull. 2014;40(1):120-131.

22. Dominguez MD, Saka MC, Lieb R, Wittchen HU, van Os J. Early expression of negative/disorganized symptoms predicting psychotic experiences and subsequent clinical psychosis: a 10-year study. The American journal of psychiatry. 2010;167(9):10751082.

23. Kelleher I, Devlin N, Wigman JT, et al. Psychotic experiences in a mental health clinic sample: implications for suicidality, multimorbidity and functioning. Psychol Med. 2014;44(8):1615-1624.

24. Honings S, Drukker M, van Nierop M, et al. Psychotic experiences and incident suicidal ideation and behaviour: Disentangling the longitudinal associations from connected psychopathology. Psychiatry Res. 2016;245:267-275.

25. Cougnard A, Marcelis M, Myin-Germeys I, et al. Does normal developmental expression of psychosis combine with environmental risk to cause persistence of psychosis? A psychosis proneness-persistence model. Psychological medicine. 2007;37(4):513527.

26. Zheutlin AB, Dennis J, Karlsson Linner R, et al. Penetrance and Pleiotropy of Polygenic Risk Scores for Schizophrenia in 106,160 Patients Across Four Health Care Systems. The American journal of psychiatry. 2019;176(10):846-855.

27. Ripke S, Neale BM, Corvin A, et al. Biological insights from 108 schizophreniaassociated genetic loci. Nature. 2014;511(7510):421-427.
28. Mistry S, Harrison JR, Smith DJ, EscottPrice V, Zammit S. The use of polygenic risk scores to identify phenotypes associated with genetic risk of bipolar disorder and depression: A systematic review. J Affect Disorders. 2018;234:148-155.

29. Zhang J-P, Robinson D, Yu J, et al. Schizophrenia polygenic risk score as a predictor of antipsychotic efficacy in firstepisode psychosis. Am J Psychiat. 2019;176(1):21-28.

30. Richardson TG, Harrison S, Hemani G, Davey Smith G. An atlas of polygenic risk score associations to highlight putative causal relationships across the human phenome. Elife. 2019;8:e43657.

31. The Brainstorm Consortium, Anttila V, Bulik-Sullivan B, et al. Analysis of shared heritability in common disorders of the brain. Science. 2018;360(6395):eaap8757.

32. Pazoki R, Lin BD, van Eijk KR, et al. Polygenic risk scores are associated with quality of life in schizophrenia. submitted.

33. Hatzimanolis A, Bhatnagar P, Moes A, et al. Common genetic variation and schizophrenia polygenic risk influence neurocognitive performance in young adulthood. Am J Med Genet B Neuropsychiatr Genet. 2015;168B(5):392-401.

34. Jones HJ, Stergiakouli E, Tansey KE, et al. Phenotypic Manifestation of Genetic Risk for Schizophrenia During Adolescence in the General Population. JAMA psychiatry. 2016;73(3):221-228.

35. Hatzimanolis A, Avramopoulos D, Arking $\mathrm{DE}$, et al. Stress-dependent association between polygenic risk for schizophrenia and schizotypal traits in young army recruits. Schizophrenia Bull. 2017;44(2):338-347.

36. Richards A, Horwood J, Boden J, et al. Associations between schizophrenia genetic risk, anxiety disorders and manic/hypomanic episode in a longitudinal population cohort study. Brit J Psychiat. 2019;214(2):96-102.

37. Sieradzka D, Power RA, Freeman D, et al Are genetic risk factors for psychosis also associated with dimension-specific psychotic 
experiences in adolescence? PloS one.

2014;9(4).

38. van Os J, Pries L-K, Delespaul P, et al. Replicated evidence that endophenotypic expression of schizophrenia polygenic risk is greater in healthy siblings of patients compared to controls, suggesting geneenvironment interaction. The EUGEI study. Psychological medicine. 2019:1-14.

39. Legge SE, Jones HJ, Kendall KM, et al. Association of Genetic Liability to Psychotic Experiences With Neuropsychotic Disorders and Traits. JAMA psychiatry. 2019;76(12):1256-1265.

40. Zammit S, Hamshere M, Dwyer S, et al. A population-based study of genetic variation and psychotic experiences in adolescents. Schizophr Bull. 2014;40(6):1254-1262.

41. Van Scheltinga AT, Bakker S, Van Haren N, et al. Schizophrenia genetic variants are not associated with intelligence. Psychological medicine. 2013;43(12):2563-2570.

42. Riglin L, Collishaw S, Richards A, et al. Schizophrenia risk alleles and neurodevelopmental outcomes in childhood: a population-based cohort study. Lancet Psychiat. 2017;4(1):57-62.

43. van Os J, van der Steen Y, Islam MA, et al. Evidence that polygenic risk for psychotic disorder is expressed in the domain of neurodevelopment, emotion regulation and attribution of salience. Psychol Med. 2017;47(14):2421-2437.

44. Nivard MG, Gage SH, Hottenga JJ, et al. Genetic Overlap Between Schizophrenia and Developmental Psychopathology: Longitudinal and Multivariate Polygenic Risk Prediction of Common Psychiatric Traits During Development. Schizophrenia Bull. 2017;43(6):1197-1207.

45. Pruessner M, Cullen AE, Aas M, Walker EF. The neural diathesis-stress model of schizophrenia revisited: An update on recent findings considering illness stage and neurobiological and methodological complexities. Neurosci Biobehav Rev. 2017;73:191-218.
46. Ursini G, Punzi G, Chen Q, et al. Convergence of placenta biology and genetic risk for schizophrenia. Nat Med. 2018;24(6):792.

47. French L, Gray C, Leonard G, et al. Early cannabis use, polygenic risk score for schizophrenia and brain maturation in adolescence. JAMA psychiatry. 2015;72(10):1002-1011.

48. Eberle C, Peterse Y, Jukic F, et al. Endophenotype Potential of Nucleus Accumbens Functional Connectivity: Effects of Polygenic Risk for Schizophrenia Interacting with Childhood Adversity. Journal of Psychiatry. 2019;4(3).

49. Trotta A, Iyegbe C, Di Forti M, et al. Interplay between schizophrenia polygenic risk score and childhood adversity in firstpresentation psychotic disorder: a pilot study. PLoS One. 2016;11(9):e0163319.

50. VanderWeele TJ, Knol MJ. A tutorial on interaction. Epidemiologic Methods. 2014;3(1):33-72.

51. Mas $\mathrm{S}$, Boloc $\mathrm{D}$, Rodríguez $\mathrm{N}$, et al. Examining Gene-Environment Interactions Using Aggregate Scores in a First-Episode Psychosis Cohort. Schizophrenia Bull. 2020.

52. Ramsay H, Kelleher I, Flannery P, et al. Relationship between the COMTVal158Met and BDNF-Val66Met Polymorphisms, Childhood Trauma and Psychotic Experiences in an Adolescent General Population Sample. PloS one. 2013;8(11).

53. van Winkel R, Stefanis NC, Myin-Germeys I. Psychosocial stress and psychosis. A review of the neurobiological mechanisms and the evidence for gene-stress interaction. Schizophr Bull. 2008;34(6):1095-1105.

54. van Nierop M, Janssens $M$, Bruggeman R, et al. Evidence that transition from health to psychotic disorder can be traced to semiubiquitous environmental effects operating against background genetic risk. PloS one. 2013;8(11):e76690.

55. Pinckaers FME, Rotee ILM, Nwosu CV, et al. Evidence for interaction between genetic 
liability and childhood trauma in the development of psychotic symptoms. Soc Psych Psych Epid. 2019;54(9):1045-1054.

56. Colodro-Conde L, Couvy-Duchesne B, Zhu $\mathrm{G}$, et al. A direct test of the diathesis-stress model for depression. Molecular psychiatry. 2018;23(7):1590.

57. Arnau-Soler A, Adams MJ, Clarke TK, et al. A validation of the diathesis-stress model for depression in Generation Scotland. Transl Psychiatry. 2019;9(1):25.

58. Arnau-Soler A, Macdonald-Dunlop E, Adams MJ, et al. Genome-wide by environment interaction studies of depressive symptoms and psychosocial stress in UK Biobank and Generation Scotland. Transl Psychiatry. 2019;9(1):14.

59. Murray RM, Lewis SW. Is schizophrenia a neurodevelopmental disorder? Br Med J (Clin Res Ed). 1987;295(6600):681.

60. Weinberger DR. The neurodevelopmental origins of schizophrenia in the penumbra of genomic medicine. World Psychiatry. 2017;16(3):225-226.

61. Radua J, Ramella-Cravaro V, Ioannidis JP, et al. What causes psychosis? An umbrella review of risk and protective factors. World Psychiatry. 2018;17(1):49-66.

62. Belbasis L, Köhler C, Stefanis N, et al. Risk factors and peripheral biomarkers for schizophrenia spectrum disorders: an umbrella review of meta-analyses. Acta Psychiat Scand. 2018;137(2):88-97.

63. Stepniak B, Papiol S, Hammer C, et al. Accumulated environmental risk determining age at schizophrenia onset: a deep phenotyping-based study. Lancet Psychiat. 2014;1(6):444-453.

64. Padmanabhan JL, Shah JL, Tandon N, Keshavan MS. The "polyenviromic risk score": aggregating environmental risk factors predicts conversion to psychosis in familial high-risk subjects. Schizophrenia research. 2017;181:17-22.

65. Guloksuz S, van Os J, Rutten BPF. The Exposome Paradigm and the Complexities of
Environmental Research in Psychiatry. JAMA psychiatry. 2018;75(10):985-986.

66. Guloksuz S, Rutten BPF, Pries LK, et al. The Complexities of Evaluating the Exposome in Psychiatry: A Data-Driven Illustration of Challenges and Some Propositions for Amendments. Schizophr Bull. 2018;44(6):1175-1179.

67. McLaughlin KA, Conron KJ, Koenen KC, Gilman SE. Childhood adversity, adult stressful life events, and risk of past-year psychiatric disorder: a test of the stress sensitization hypothesis in a populationbased sample of adults. Psychol Med. 2010;40(10):1647-1658.

68. Mansueto G, Faravelli C. Recent life events and psychosis: The role of childhood adversities. Psychiatry Res. 2017;256:111117.

69. Holtzman CW, Trotman HD, Goulding SM, et al. Stress and neurodevelopmental processes in the emergence of psychosis. Neuroscience. 2013;249:172-191.

70. Kendler KS, Kuhn JW, Prescott CA. Childhood sexual abuse, stressful life events and risk for major depression in women. Psychological medicine. 2004;34(8):14751482.

71. Morgan C, Reininghaus U, Fearon P, et al. Modelling the interplay between childhood and adult adversity in pathways to psychosis: initial evidence from the AESOP study. Psychological Medicine. 2014;44(2):407419.

72. Ayesa-Arriola R, Setien-Suero E, MarquesFeixa $L$, et al. The synergetic effect of childhood trauma and recent stressful events in psychosis: associated neurocognitive dysfunction. Acta Psychiatr Scand. 2019:1-9.

73. Lataster J, Myin-Germeys I, Lieb R, Wittchen HU, Van Os J. Adversity and psychosis: a 10-year prospective study investigating synergism between early and recent adversity in psychosis. Acta Psychiat Scand. 2012;125(5):388-399.

74. ten Have M, de Graaf R, Van Dorsselaer S, Tuithof M, Kleinjan M, Penninx BW. 
Childhood maltreatment, vulnerability characteristics and adult incident common mental disorders: 3-year longitudinal data among> 10,000 adults in the general population. Journal of psychiatric research. 2019;113:199-207.
75. Colodro-Conde L, Couvy-Duchesne B, Whitfield JB, et al. Association between population density and genetic risk for schizophrenia. JAMA psychiatry. 2018;75(9):901-910 






APPENDICES

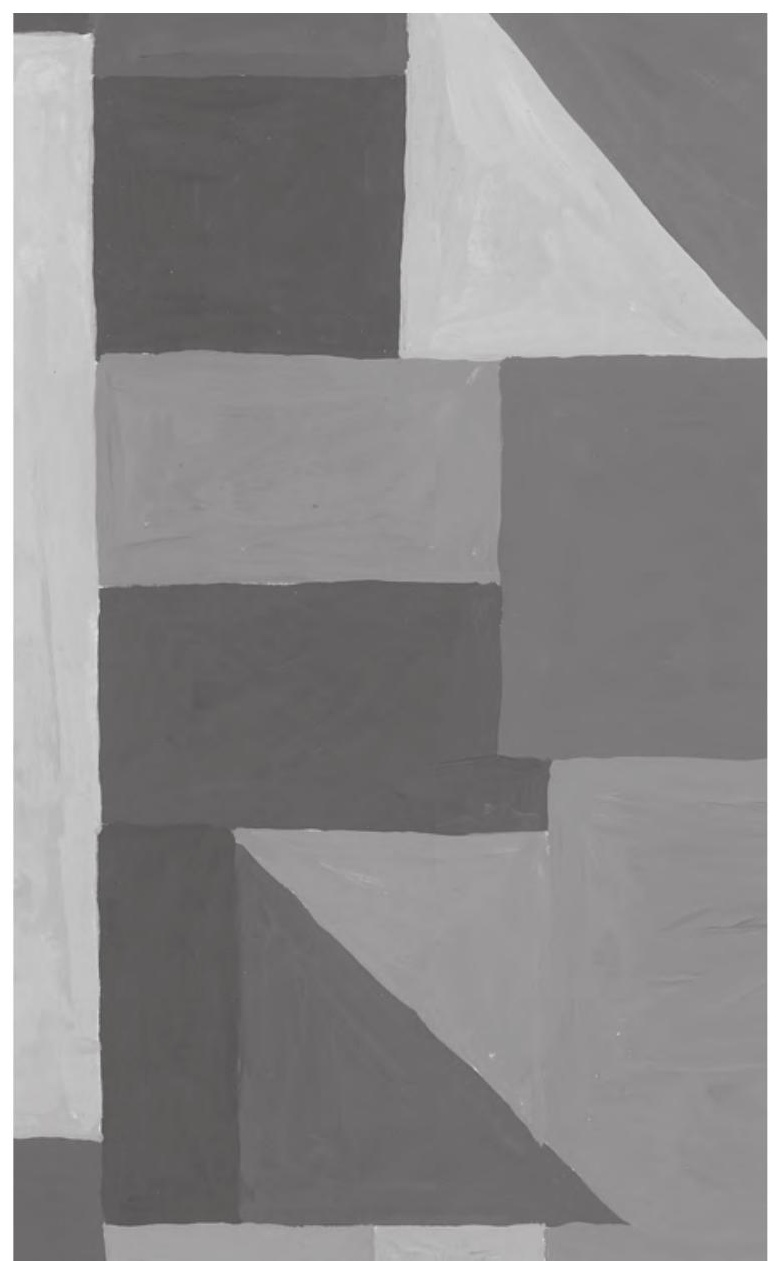





\title{
12.1 Resilience against traumatic stress: current developments and future directions
}

\author{
Clara Snijders*, Lotta-Katrin Pries*, Noemi Sgammeglia, Ghazi Al Jowf, Nagy A. Youssef, \\ Laurence de Nijs, Sinan Guloksuz, Bart P.F. Rutten
}

Frontiers Psychiatry (2018): 9:676

*Equally contributed

\begin{abstract}
Given the high prevalence of stress-related mental disorders, their impact on person, family, and society and the paucity of treatment options for most of these disorders, there is currently a pressing need for innovative approaches to deal with these issues and enhance well-being. One approach which has received increasing attention over the last decade is to shift our scientific and clinical focus from risk factors for psychopathology to factors promoting resilience and mental well-being. In order to summarize and evaluate the current state of scientific affairs on the biological basis of resilience, we provide an overview of the literature on animal and human studies of resilience. Because resilience can only truly be operationalized through longitudinal data collection and analyses, we focus primarily on longitudinal studies. This review shows that the concept of resilience is currently being operationalized, measured and even defined in widely variable manners, both within animal and human studies. We further provide an overview of existing and new strategies that could help promote resilience and which are proposed to be implemented more often in clinical situations. Finally, we summarize the challenges the field is facing and provide recommendations for future research.
\end{abstract}

Keywords: resilience, stress, prospective longitudinal studies, resilience-promoting interventions, review 


\section{Introduction}

Over the past decade, research on resilience has received increasing attention. The heightened interest in understanding and promoting resilience is not surprising given that in Europe alone, anxiety disorders and major depressive disorder were among the most frequent mental disorders in 2011 with a 12 -month prevalence of $14 \%$ (corresponding to 61.5 million individuals) and $6.9 \%$ (30.3 million), respectively1. This is not much different than the prevalence of these disorders in North America and many other parts of the world. Moreover, a recent meta-analysis showed that relapse rates in patients suffering from depression remain high and that long-term effects of conventional treatment options are not always encouraging2. These findings call for additional strategies and alternative interventions needed to prevent disease development and boost resilience.

Here, we conceptualize resilience as being an active and dynamic process through which a person adaptively overcomes a stressful or difficult situation or recovers swiftly from a period of ill-health ${ }^{3}$. Thus, resilience is not a passive reaction to an adverse situation, nor is it merely the reverse side of post-traumatic stress disorder (PTSD) or the absence of symptomatology $y^{34}$. Research on resilience is facing several challenges of which the most fundamental one consists of the enormous heterogeneity in defining and operationalizing resilience. Indeed, some researchers will conceptualize resilience as being a personality trait while others will use the terms outcome, coping strategy or dynamic process (see ${ }^{5}$ for a critical overview of different theories on resilience).

Such discrepancies impede its measurement and further hinder the comparison of obtained results across resilience studies. Therefore, the following proposals have recently been formulated to guide future resilience research; (i) the fast recovery of mental health following stress exposure reflects a dynamic adaptation process, resilience should not be understood as a personality trait, the result of a specific genotype or other hardwired characteristics, there is a strong need for prospective longitudinal studies, and (iv) resilience can only be operationalized following a stressful period or event ${ }^{3}$. Such stressors can refer to various types of (potentially) traumatic or life-threatening events, injury, illness, or difficult life circumstances such as unemployment, grief or divorce. Although most individuals recover promptly following trauma exposure, gaining a deeper understanding of the mechanisms underlying resilience could be of use to those more at risk of developing a stress-related disorder. 
Following the conceptualization of resilience as proposed by Kalisch et al. ${ }^{3}$, the main aim of the present review is to summarize the findings from key longitudinal animal and human studies on resilience. Furthermore, we propose strategies aiming to promote resilience, discuss limitations and challenges of current resilience research, and suggest future directions to help this field evolve.

\section{Resilience studies}

Over the past years, most resilience studies used cross-sectional designs. However, susceptibility and resilience to past or ongoing stress are hard to capture when assessing mental health at one time point only. Moreover, these designs do not allow for the exclusion of baseline differences between subjects which further impairs obtaining a comprehensive interpretation of the obtained findings. Using a longitudinal design enables one to assess dynamic behavioral and biological fluctuations over time, enabling investigations of (baseline) predictors of differential susceptibility to future stress. This is highly relevant, especially in light of at-risk jobs where trauma exposure is more prevalent. In order to take this into account, the first part of this review only includes prospective longitudinal animal and human studies, i.e., studies in which the individuals mental health or the animals behavioral or physiological state were quantitatively assessed before the (natural or experimental) stress exposure and at least once after this period. Expanding on the review by Kalisch et al. ${ }^{3}$, the literature was searched for studies published in 2017 which fulfilled several criteria. First, we only included studies in which the severity and duration of the stress exposure was precisely quantified. For the human studies specifically, we only included studies in which the baseline assessments of predictors of mental health were recorded. In addition, and as postulated by Kalisch et al. $^{3}$, in susceptible individuals, the severity of the stress exposure had to positively correlate with the development of mental health problems. Studies were also only considered when including adults and the study groups consisted of 30 subjects or more ${ }^{3}$. Since stress responses in children vary depending on their developmental stage, discussing these findings would require a different focus which does not fit within the scope of the present review. Finally, it needs to be noted that no systematic search of the literature was performed given the wide variety of terms used by researchers to describe resilience. 


\section{Animal studies}

In animal research, resilient phenotypes are often identified based on specific behavioral outcomes following a well-defined period of experimentally induced stress. Specifically, those animals showing a fast recovery from a stressful manipulation are said to be resilient. Although caution and critical evaluation of the observed phenotypes are needed when interpreting and translating the obtained findings, these models can provide us with some of the molecular underpinnings of differential susceptibility to stress. Using the abovementioned eligibility criteria, we identified six studies which can be found in Table 1. Studies sharing similar features are discussed together in the next paragraphs.

\section{Inflammatory Markers}

One study examined acoustic startle responses (which has been related to PTSD) in rats before and after exposure to a single episode of inescapable footshocks and 1-min reminders for the next 6 weeks ${ }^{10}$. Rats exhibiting a high baseline startle response showed a significantly higher startle response following the period of exposure. Interestingly, rats showing an increased startle response at baseline also exhibited elevated plasma corticosterone levels at follow-up as compared to the rats with low baseline startle responses (although it should be noted that corticosterone levels were not measured prior to the experimental manipulation). Links between increased corticosterone levels and stress-induced behavioral phenotypes have also been observed in other studies ${ }^{8,9}$. One of these studies investigated whether longitudinal changes in blood corticosterone levels were associated with measures of differential susceptibility to stress in mice ${ }^{8}$. The authors found that mice with an increase in plasma corticosterone levels upon 2 weeks of repeated restraint stress exposure showed significant weight loss over the course of the experiment as well as anxiety-related behaviors at follow-up as measured through the Elevated Plus Maze (EPM) and Open Field Test (OFT). These mice were thus characterized as being susceptible while more resilient mice were identified when showing (i) a decrease in corticosterone levels from baseline to follow-up, (ii) a stable body weight, and (iii) no anxiety-related behavior. Furthermore, the authors found that corticosterone levels at baseline predicted the extent of change in corticosterone levels during stress exposure and correlated with behavioral measures at follow-up. Another study used a repeated social defeat stress paradigm of 10 days and showed that those mice that later became susceptible to stress exposure had higher baseline levels of leukocytes ${ }^{7}$. Moreover, in response to acute stress and prior to the repeated stress exposure paradigm, the same mice exhibited higher levels of the pro-inflammatory cytokine interleukin-6 (IL-6). Together, these results suggest that baseline levels of specific inflammatory markers might predict differential susceptibility to future stress exposure in mice. 
Table 1 Longitudinal animal studies assessing biological outcomes associated with differential susceptibility to stress

\begin{tabular}{|c|c|c|c|c|}
\hline References & Species and sex & Stressor & $\begin{array}{c}\text { Outcome of } \\
\text { interest }\end{array}$ & Main finding \\
\hline Chen et al. ${ }^{6}$ & $\begin{array}{l}\text { Male Sprague- } \\
\text { Dawley rats }\end{array}$ & $\begin{array}{c}7 \text { days of chronic social } \\
\text { defeat stress }\end{array}$ & $\begin{array}{l}\text { Circulating } \\
\text { miRNAs (tail } \\
\text { blood) }\end{array}$ & $\begin{array}{l}\downarrow \text { Pre-stress miR-24-2-5p, miR-27a- } \\
\text { 3p, miR-30e-5p, miR-362-3p, } \\
\text { associates with future vulnerability to } \\
\quad \text { chronic social stress. } \\
\downarrow \text { Post-stress miR-139-5p, miR-28- } \\
\text { 3p, miR-326-3p, miR-99b-5p } \\
\text { associates with ongoing resilience. }\end{array}$ \\
\hline
\end{tabular}

Hodes et al. ${ }^{7}$ Male CD 45.1+/CD 10 days of repeated $45.2+\mathrm{C} 57 \mathrm{BL} / 6 \quad$ social defeat stress mice
Kim et al. ${ }^{8} \quad$ Male C57BL/6N Chronic restraint stress mice

Magalhaes et Male Wistar rats al. ${ }^{9}$

Rasmussen et Male Wistar rats al. ${ }^{10}$

Tse et al. ${ }^{11}$
3 weeks of chronic

unpredictable stress

Inescapable footshock

$$
\text { + weekly 1-min }
$$

reminders for 6 weeks

Plasma
corticosterone

Neuroimagingfunctional connectivity and structural changes

Acoustic startle $\uparrow$ Pre-stress acoustic startle response response $=\uparrow$ post-stress plasma corticosterone
levels and $\uparrow$ post-stress acoustic
startle response.

Hippocampal $\uparrow$ Post-stresslefthippocampalvolume volume
Longitudinal changes in corticosterone reflect differential stress susceptibility and pre-stress corticosterone predicts post-stress susceptibility or resilience.

Pre-stress differences in functional connectivity in brainstem-limbic area between susceptible and resilient rats. 


\section{MicroRNAs}

Only one prospective study examined the potential of microRNAs (miRNAs) to distinguish resilient and vulnerable animals ${ }^{6}$. miRNAs are small, non-coding RNAs which are involved in the post-transcriptional regulation of gene expression ${ }^{12}$. miRNAs have been widely studied in cancer and cardiovascular disease as potential biomarkers, but less is known regarding their involvement in mental disorders. ${ }^{6}$ examined whether miRNAs could serve as biomarkers of resilience or vulnerability to stress by using a chronic social defeat paradigm in rats that lasted for 7 days. Rats showing little or no avoidance behavior when encountering an unfamiliar rat in its own cage were identified as being resilient while susceptible rats spent less time interacting with the novel rat as compared to controls. The authors found that susceptible rats had lower baseline blood circulating levels of miR-24-2-5p, miR-27a-3p, miR-30e-5p, and miR362-3p compared to unexposed controls. However, the more resilient rats had lower levels of blood circulating levels of miR-139-5p, miR-28-3p, miR-326-3p, miR-99b-5p at follow-up as compared to controls. At both time points, no differences in miRNA expression were found between resilient and susceptible animals. These results pinpoint a number of candidate microRNA species which could, at least in part, regulate vulnerability to future stress or reflect ongoing resilience to chronic social stress in rats.

\section{Neuroimaging Data}

Using magnetic resonance imaging (MRI) in mice, Tse et al. were the first to assess changes in hippocampal volume prior to and following stress exposure ${ }^{11}$. The authors identified susceptible and resilient animals based on their behavioral profiles in the social defeat paradigm. Following stress exposure, approximately half of the mice were classified as susceptible to stress, while the other half was more resilient. In contrast to the susceptible mice which showed no hippocampal volume increase over time, resilient and non-stressed control mice showed an increase in the left hippocampal volume from baseline to post-stress exposure, suggesting that normal hippocampal growth was impaired in susceptible animals only. Intriguingly, a positive correlation was observed between hippocampal volume at baseline and social avoidance behavior at follow-up. These findings suggest that differences in hippocampal volumes could be associated with vulnerability to future stress in mice, which is further supported by similar findings in humans ${ }^{13}$.

More recently, another study assessed structural changes more broadly along with alterations in the brain's functional connectome upon stress exposure? ${ }^{9}$ In this study, rats underwent a chronic unpredictable stress protocol for 3 weeks. Blood corticosterone levels, MRI scans and anxiety-related behaviors (measured through the EPM) were measured 
before, 7 and 21 days after exposure to stress. The authors categorized the stress-exposed rats into susceptible and resilient groups based on aberrant behavior and plasma corticosterone levels. Those rats expressing lower levels of post-exposure corticosterone in combination with minimal anxiety-like behavior were categorized as being resilient. Among a broad variety of structural and functional alterations induced by stress, it was found that baseline differences in functional connectivity measures of a specific brainstem-limbic network were able to distinguish the resilient and susceptible groups, with susceptible rats showing lower functional connectivity compared to the resilient ones. It is worth mentioning that in humans, distinct patterns of brain activity have also been linked to PTSD and treatment response $\mathrm{e}^{14-16}$. Together, these results suggest that imaging data can contribute to a better understanding of the psychopathology of PTSD and potentially serve as a predictive biomarker of future vulnerability to stress and/or treatmentresponse.

\section{Human studies}

Various collaborative prospective approaches are currently being conducted and followed-up on, including longitudinal approaches such as PRISMO ("Stressgerelateerd Militia Onderzoek" $)^{17-21}$, the Marine Resiliency Study (MRS) I and II ${ }^{22-24}$, and the prospective-longitudinal component of the Prevalence, Incidence and Determinants of PTSD and Other Mental Disorders (PID-PTSD $\left.{ }^{+3}\right)^{25,26}$. Following our eligibility criteria, 12 articles were identified. All dealt with deployment-related stress, i.e., long-lasting effects of events experienced during actual combat, assault or other living conditions experienced during deployment ${ }^{3}$. Studies sharing similar features are discussed together in the following paragraphs and can be found in Table 2 . 
Table 2 Longitudinal human studies assessing biological outcomes associated with differential susceptibility to stress

\begin{tabular}{|c|c|c|c|c|}
\hline References & Sample (N) & $\begin{array}{c}\text { Main } \\
\text { stressor }\end{array}$ & $\begin{array}{l}\text { Outcome of } \\
\text { interest }\end{array}$ & Main finding \\
\hline \multicolumn{5}{|l|}{ GENETIC } \\
\hline \multicolumn{5}{|l|}{ FACTORS } \\
\hline Clark et al. ${ }^{27}$ & $\begin{array}{l}\text { RINGS; Male soldiers: } \\
\qquad N=253\end{array}$ & Deployment & PTSD & $\begin{array}{c}\text { Met/Met and } \mathrm{Val} / \mathrm{Val} \text { genotypes } \\
\text { had stronger trauma-responses } \\
\text { than the } \mathrm{Val} / \text { Metgenotype. }\end{array}$ \\
\hline Wald et al. ${ }^{28}$ & $\begin{array}{l}\text { Israeli Defense Force; Male } \\
\text { soldiers: } \\
\qquad N=1,085\end{array}$ & Deployment & PTSD & $\begin{array}{l}\text { Threat bias interacted with } \\
\text { combat exposure and threat bias } \\
\text { interacted with combat exposure } \\
\text { and 5-HTTLPR. }\end{array}$ \\
\hline
\end{tabular}

\section{EPIGENETIC}

\section{FACTORS}

\begin{tabular}{|c|c|c|c|c|}
\hline & PRISMO and MRS; Male & & & Genome-wide changes at 17 \\
\hline Rutten et al. ${ }^{20}$ & $\begin{array}{c}\text { soldiers/marines: } \\
N=93, N=98\end{array}$ & Deployment & PTSD & $\begin{array}{l}\text { positions and } 12 \text { regions were } \\
\text { associated with PTSD status. } \\
\text { Pre-deployment GR-1F region }\end{array}$ \\
\hline Schur et al. ${ }^{21}$ & $\begin{array}{l}\text { PRISMO, Male soldiers: } \\
\qquad N=92\end{array}$ & Deployment & $\begin{array}{c}\text { Mental health and } \\
\text { PTSD }\end{array}$ & $\begin{array}{l}\text { (52 loci) methylation did not } \\
\text { predict mental health or PTSD } \\
\text { status. }\end{array}$ \\
\hline Van Zuiden et al. ${ }^{17}$ & $\begin{array}{l}\text { PRISMO Male soldiers: } \\
\qquad N=68\end{array}$ & Deployment & PTSD & $\begin{array}{l}\text { mRNA expression of GR- } \alpha \text {, } \\
\text { GR-P, GR- } \beta \text {, glucocorticoid- } \\
\text { induced leucine zipper (GILZ), } \\
\text { serum and glucocorticoid- } \\
\text { inducible kinase-1 (SGK-1), or } \\
\text { FKBP5 does not predict PTSD } \\
\text { status. }\end{array}$ \\
\hline
\end{tabular}

\section{CIRCULATING}

MARKERS

\section{Inflammatory}

Markers

MRS II and MRS; Male

Breen et al. ${ }^{23}$ marines: Deployment

$$
N=124, N=50
$$

Eraly et al. 22
MRS, Male marines:

$$
N=1,719
$$

PTSD status associated with gene co-expression networks related to innate immune responses.

Baseline plasma levels of Creactive protein $(\mathrm{CRP})$ predicted PTSD symptoms. 




MRS, MRS II, MarineResiliencyStudy I, II; PID-PTSD ${ }^{+3}$, Incidence and Determinants of PTSD and Other Mental Disorders; PRISMO, StressgerelateerdMilitia Onderzoek;RINGS, The Readiness and Resilience in National Guard Soldiers Study. Within each section, studies were listed in alphabetical order based on the surname of the first author.

\section{Genetics}

Two studies examined the longitudinal effects of genetic variations on stress-related PTSD symptoms. Both studies focused on candidate genes, i.e., the serotonin transporter (5-HTT) gene and catechol-O-methyltransferase (COMT) gene, which are known to affect serotonergic and dopaminergic signaling, respectively. 
In the first study, the effects of a serotonin transporter gene-linked polymorphic region [5HTTLPR] and threat-related attention on post-deployment PTSD symptoms was evaluated in 1,085 male soldiers ${ }^{28}$. PTSD symptoms and threat-related attention bias (measured with a computerized dot-probe task) were assessed three times with the last assessment taking place around 1 year after baseline. Combat exposure between the baseline and follow-up assessments was inferred by using geo-operational exposure data and self-report measures [i.e., the Combat Experiences Scale with two additional items ${ }^{29}$ ]. The authors observed that pre-deployment-threat bias interacted with combat exposure during deployment and 5HTTLPR in predicting post-deployment PTSD symptoms. More specifically, fewer postdeployment PTSD symptoms after high combat exposure were found in those individuals who displayed pre-deployment threat vigilance and had the SS/SL-G alleles (i.e., reflecting low transcription 5-HTT) of the 5-HTTLPR genotypes. This study is particularly interesting in highlighting the complex interaction between context, stress-exposure, attention bias and genetics, suggesting that serotonergic transmission may be involved in the co-occurrence of avoidance and hypervigilance symptoms in $\mathrm{PTSD}^{30}$.

In another study, 253 Iraq war veterans were assessed prior to and following a deployment period of 16 months ${ }^{27}$. Deployment trauma was measured with the Post Deployment Stressors subscale of the Deployment Risk and Resilience Inventory (DRRI) ${ }^{31}$ as well as by using one additional item on sexual assault experienced during deployment. DNA was extracted from blood or buccal swabs and was genotyped into COMT Met/Met $(n=63)$, $\mathrm{Val} / \mathrm{Met}(\mathrm{n}=131)$, or $\mathrm{Val} / \mathrm{Val}(\mathrm{n}=42)$. Regression analyses showed that the effect of deployment trauma on PTSD was dependent on COMT polymorphism with carriers of the homozygous genotypes (Met/Met and $\mathrm{Val} / \mathrm{Val}$ ) showing more PTSD symptoms than those carrying the heterozygous (Val/Met) genotype. This is in line with previous human and animal studies which highlight the role of the Met/Met genotype and show some preliminary support for the $\mathrm{Val} / \mathrm{Val}$ genotype as a risk factor for the development of $\mathrm{PTSD}^{32-34}$.

\section{Epigenetics}

Several cross-sectional studies support the putative role of epigenetic mechanisms, especially DNA methylation, in the impact of traumatic stress on mental health 35-38. Recently, prospective epigenetic studies have started to investigate the links between changes in PTSD symptom scores and changes in epigenetic profiles across the period of exposure to traumatic stress. These studies were conducted in subsamples of the PRISMO project and focus on the glucocorticoid receptor exon $1 \mathrm{~F}(\mathrm{GR}-1 \mathrm{~F})$ region and the predictive role of epigenetic markers in PTSD. In the first study, methylation signatures of the GR-1F region (52 loci) were quantified in peripheral blood cells of 92 Dutch military personnel which were collected 
before and after a 4-month deployment period to Afghanistan. More specifically, the authors focused on mean methylation across all cytosine- phosphate-guanines ( $\mathrm{CpGs}$ ), the number of methylated loci and those CpGs of which methylation was known from previous publications to be associated with GR exon 1F mRNA expression. The latter was termed "functional methylation". It was found that an increase in either of the methylation levels (i.e., mean, number, and functional) within this region was associated with increases in PTSD symptom scores in trauma-exposed subject. Increased functional methylation was associated with mental health. However, PTSD and mental health problems occurring 6 months post-deployment within individuals exposed to trauma were predicted by neither of the pre-deployment methylation levels (i.e., mean, number, or functional) ${ }^{21}$.

In a recent prospective epigenetic study performed using two military cohorts ${ }^{20}$, the impact of traumatic stress during combat on post-deployment PTSD symptoms and associated longitudinal epigenetic changes was investigated. In a discovery sample of 93 male Dutch servicemen [PRISMO cohort; same subjects as Schur et al. ${ }^{21}$ ], specific DNA methylation alterations were associated with the development of PTSD. This cohort displayed changes at 17 positions and 12 regions and subsequent bioinformatic analyses highlighted the role of different pathways linked to PTSD symptomatology. Interestingly, the associations between the development of PTSD symptoms and decreased DNA methylation at genomic regions in ZFP57, RNF39, and HIST1H2APS2 were replicated in a male US marine cohort of MRS with a 7-month war-zone deployment to Iraq or Afghanistan $(\mathrm{n}=98)$. It is worth mentioning that international efforts such as the Psychiatric Genomics Consortium (PGC) PTSD group, which includes data on a large combined sample of four military studies and three civilian cohorts $(\mathrm{N}=1,147)$, might have increased statistical power to detect further relevant epigenetic variations and thereby provide deeper insights in the near future ${ }^{39}$.

\section{Blood Markers}

\section{Inflammatory Markers}

Upon the observation that PTSD co-occurred with peripheral inflammation in crosssectional studies, the question arose as to whether inflammatory markers are causally involved in the disorder or are one of its consequences ${ }^{40}$. Since then, several prospective studies have attempted to evaluate the causal role of various inflammatory responses in the development of PTSD.

One study used a subset of the MRS dataset $(\mathrm{N}=1,719)$ and found that in U.S. Marines, baseline plasma levels of C-reactive protein (CRP) was a strong predictor of post- deployment PTSD symptoms ${ }^{22}$. Another study analyzed gene co-expression profiles obtained through 
RNA sequencing of peripheral blood leukocytes from Marines belonging to the MRS II ( $\mathrm{N}=124)$ and replicated the obtained findings in a separate subsample of the MRS $(\mathrm{N}=50)$. It was found that both at pre- and post-deployment, co-expression gene networks linked to the innate immune responses, interferon signaling, and monocyte specificity were predictive of post-deployment PTSD ${ }^{23}$. Following this and using the same sample as Breen et al. ${ }^{23}$, researchers ${ }^{24}$ aimed to build upon these findings and ascertained several master regulators driving the previously identified networks. Using ARACNe (Algorithm for Reconstruction of Accurate Cellular Networks) and protein activity analysis they identified SOX3, TNFAIP3, TRAFD1, POU3F3, STAT2, and PML as important master regulators. Gene Ontology analyses enriched by TNFAIP3, TRAFD1, and PML again pointed toward the role of the innate immune responses in the development of PTSD.

In a subsample of the PRISMO dataset $(N=693)$, researchers addressed the immune activation by measuring in vitro cytokine production by leukocytes upon stimulation ${ }^{18}$. Among other findings, the authors observed a three-way interaction between cytokine production at 1-month post-deployment, trauma exposure during combat (assessed 1-month post-deployment), and post-deployment stressful life events during 12 months postdeployment on the longitudinal changes in PTSD symptoms scores as measured between 1 month and 2 years post deployment. More specifically, increased mitogen-stimulated T-cell and innate cytokine production, greater exposure to stress during combat and during the 12 month post-deployment period were associated with increased PTSD symptoms between 1 month and 2 years post-deployment.

\section{Hormonal Dysregulations}

Another line of studies focused on the functioning of the hypothalamic-pituitary-adrenal (HPA) axis. In this regard, three studies investigated the links between cortisol levels and stress exposure on the development of PTSD. Two of these studies were part of the PID$\mathrm{PTSD}^{+3}$ project and assessed hair cortisol concentration (HCC) and cortisol stress reactivity, measured through saliva cortisol levels before and after the Trier Social Stress Test (TSST), prior to and following a deployment period of $\sim 5$ months $s^{25}$. 26 Their main finding showed that when exposed to trauma, a lower baseline HCC and lower cortisol stress level were predictive of higher post-deployment PTSD symptomatology ${ }^{25}$ while lower HCC predicted higher daily alcohol consumption26. In another study done in the PRISMO cohort $(\mathrm{N}=455)$, plasma cortisol levels at baseline did not predict PTSD status 6 months after a 4 months deployment period17. Next to cortisol, these researchers further investigated other crucial molecules of the HPA axis. van Zuiden et al. evaluated the predictive role of mRNA expression of GR- $\alpha$, GR-P, GR- $\beta$, glucocorticoid-induced leucine 
zipper (GILZ), glucocorticoid-inducible kinase-1 (SGK-1), or FKBP5 in peripheral blood mononuclear cells (PBMCs) and the number of GRs in PBMCs on post-deployment PTSD status. Interestingly, only the number of GRs in PBMCs predicted post-deployment PTSD status ${ }^{17}$.

Other researchers assessed whether plasma oxytocin (pOT) and arginine vasopressin (pAVP) levels could be used as biomarkers for stress-related development of PTSD ${ }^{19}$ in PRISMO. By investigating a group of 907 military subjects, no effects of pOT and pAVP on postdeployment PTSD was observed ${ }^{19}$.

Together, these studies highlight the value of prospective studies in linking circulating markers with the development of PTSD. While the first line of evidence suggests that elements of the immune system emerge as candidate biomarkers, there is apparent need for replications and larger longitudinal studies to confirm and extend these initial findings.

\section{Promoting Resilience}

The previous sections provided an overview of prospective human and animal studies that aimed at gaining knowledge of the mechanisms underlying mental illness and resilience. Research in this field has also turned toward studying strategies which could potentially promote mental health and boost resilience. As postulated by McEwen et al., the notion that the brain holds the ability to successfully adapt to changing environments throughout the life course, encourages one to develop top-down interventions encompassing mind-body interactions in order to install fundamental changes in various aspects of one's sense of wellbeing ${ }^{41}$. Given the previously mentioned need to expand alternative add-on strategies in order to promote resilience in todays society, this section will cover a range of psychological, behavioral and lifestyle interventions which aim to do so.

\section{Mindfulness and Meditation}

Over the last few decades, meditation techniques such as loving-kindness meditation and mindfulness meditation have been spreading in the western world. Today, mindfulness mainly owes its popularity to Professor Jon Kabat-Zinn who reintroduced it in his mindfulness-based stress reduction (MBSR) program. Described as the awareness that arises through paying purposeful and non-judgemental attention to the present moment ${ }^{42}$, mindfulness is now employed as part of standardized programs aiming to promote general human well-being and install deeply rooted positive emotions.

Empirical evidences about the benefits of mindfulness-based programs are inciting a growing interest in the (neuro)biological underpinnings of mindfulness. Different mindfulness 
programs have shown to impact both gray and white matter density of several brain structures such as in the right basolateral amygdala ${ }^{43}$ and bilateral clusters within the brainstem including the pontine tegmentum, locus coeruleus, nucleus raphe, and the sensory trigeminal nucleus ${ }^{44}$. Moreover, findings show that other types of mind-body interventions also influence various parameters of the immune system. For example, one study showed that following a yogic meditation, the activity of the proinflammatory nuclear factor-kappa beta $(\mathrm{NF}-\mathrm{\kappa B})$, known to have a prominent role in inflammation and stress, was reduced in peripheral blood leukocytes as compared to baseline measures ${ }^{45}$. Another study found an increase in telomerase activity along with reduced levels of another inflammatory marker, $\mathrm{CRP}$, in peripheral blood mononuclear cells (PBMCs) following the same type of meditation ${ }^{46}$. Yet another study found that by measuring gene expression in peripheral blood prior to and following a deep relaxation session, the expression of genes associated with telomere maintenance were enhanced at follow-up while specific genes linked to stress-related pathways were reduced in expression ${ }^{47}$. Although interesting, it is important to note that currently performed studies vary greatly in terms of the type of (mindfulness) meditation used along with the research designs and (often small) sample sizes. Gaining knowledge in the mechanisms underlying the well-documented stress-reducing and mood-enhancing effects of meditation and mindfulness-based programs ${ }^{48-51}$ holds the potential to further help the design of powerful strategies in healthcare settings to promote the cultivation of a healthy mind.

\section{Cognitive Behavioral Therapy-Based Programs}

Cognitive behavioral therapy (CBT) was originally developed by Aaron Beck to promote mental wellness and coping resources in patients suffering from mental distress such as depression, anxiety and chronic stress ${ }^{52}$. The idea of CBT is to modify one's thinking and behavioral patterns which color the way life events are being experienced. Interestingly, combining CBT with pharmacological treatments such as cognitive enhancers (but not anxiolytics) has been shown to improve long-term treatment efficacy and fear extinction, potentially by enhancing memory consolidation (recently extensively reviewed in Singewald et al. ${ }^{53}$ ). In addition, conducting CBT sessions such as exposure therapy before sleep has also been suggested to enhance treatment efficacy, raising the question whether pharmacological approaches can be implemented to enhance memory consolidations during sleep specifically ${ }^{53}{ }^{54}$. Another intervention targeting memory consolidation involves playing a computer game with high visuospatial demands (e.g., Tetris) during the hours following a traumatic event. Interestingly, this approach has recently been suggested to disrupt the consolidation of trauma memories and lead to fewer intrusive visual memories of the traumatic event ${ }^{55}$. In order to maximize treatment outcome, 
combinations of different behavioral approaches with or without various pharmacological options will need further testing.

Besides its well-documented therapeutic effect in treating mental distress and some other disorders, today's interest in CBT is also geared toward the construction of a personal model to boost resilience in the face of life's obstacles without necessarily targeting a particular mental disorder. Padesky and Mooney have proposed a CBT-based program entirely oriented toward "resiliency" research ${ }^{56}$. Their so-called strengths-based CBT consists of four sessions in which the therapist and the client actively collaborate to explore and reinforce positive qualities such as interpersonal competences, self-efficacy or self-esteem. The therapist and client co-create a personal model of resiliency by turning the previously identified strengths into effective strategies that can be applied in everyday situations. Another CBT-based program is the Stress Inoculation Therapy (SIT) which was first introduced in $1985^{57}$. In social psychology, the concept of inoculation refers to the preventive effect of brief and moderately challenging stress exposures on one's reaction to subsequent, more intense stressors. More specifically, exposing animals or individuals to minor stressors has been shown to enhance one's resilience or "inoculate" them from later stressful situations ${ }^{58-60}$. SIT incorporates this notion of inoculation in a psychotherapeutic intervention that combines cognitive and behavioral methods emphasizing coping skills learning. During a SIT session, an individual is exposed to and learns to cope with increasing amounts of stress through productive thoughts, mental images, self-statements, and relaxation training thereby enhancing his or her immunity to stress. Empirical evidence shows that SIT efficiently reduces stress, anxiety and depression in cancer patients ${ }^{61}$ and effectively reduces psychological distress up to 3 months following the sessions when delivered through two half-day training sessions in the workplace ${ }^{62}$.

\section{Physical Activity}

It is commonly known that practicing regular physical exercise leads to a plethora of positive health effects ${ }^{63}$. These benefits not only include cardiovascular and metabolic effects, but also improvements in cognitive abilities. Previous studies on animals and humans have revealed increases in synaptic plasticity and neurogenesis ${ }^{64}$, strengthened cortical activation when performing a cognitively challenging task ${ }^{65}$ and improvements in learning, slowing the course of cognitive decline in aging ${ }^{65,66}$. Using a within-subject design and multiple momentary assessments collected through experience sampling method (ESM), a diary technique assessing daily moods and activities, one study provides support for a causal effect of physical activity on positive affect ${ }^{67}$. Since the extent of positive affect levels varied between individuals based on their history of clinical depression, such findings call for individually 
tailored interventions in which clinicians could adapt the amount of physical exercise. Other studies showed that compared to training exercises with no cognitive component, specific exercises that promote mindfulness by means of calming techniques and cognitive strategies such as yoga or pilates were more effective in eliciting psychological benefits such as mood enhancements and improved executive functions over time ${ }^{68-70}$. Mindful-based physical activities, thus, seem to help improve breathing rate and depth ${ }^{71,72}$ along with heart rate ${ }^{72}$ while lowering arousal levels ${ }^{73}$. Further comparative trials in populations at high risk of robust exposure to traumatic stress are needed in order to prospectively assess the putative protective properties of these interventions on trauma-related mental ill-health.

\section{Social Support}

Several lines of evidence confirm the importance of pursuing cognitive and social activities to maintain global mental and functional health ${ }^{74-76}$. However, the exact biopsychological mechanisms underlying the positive impact of social support on mental well-being and resilience to stress still remain unclear ${ }^{77}$. To enhance both cognitive and social aspects, programs such as the Experience Corps have been introduced. This intergenerational program was originally designed by Fried et al. in 1997 to promote health among the aging population. Specifically, this program encourages adults over the age of 50 to share their skills with children needing help at school. While students obtain greater academic outcomes, older adults get the opportunity to enrich their lives on a social and cognitive level ${ }^{78}$. A recent study shows that this program further significantly slows the normal age-related decrease in cortical and hippocampal brain volumes ${ }^{79}$.

\section{Meaning and Purpose in Life}

Programs such as the Experience Corps offer older adults a sense of meaning and purpose in life which is a crucial component of mental health. A meta-analysis found that purpose in life was strongly linked to social integration in a population of older individuals and was further related to factors such as quality of life, a better health, and socioeconomic status ${ }^{80}$. Other studies have suggested that finding a sense of purpose in life is a partial mediator of the observed negative association between trait mindfulness and outcomes such as anxiety and depressive symptoms ${ }^{81}$. Having meaning to life has further been negatively associated with suicidal ideation ${ }^{82,83}$ while being an important predictor of depressive symptoms ${ }^{84}$. Taken together, these findings highlight the importance of establishing personal values and long-term goals in order to help one overcome or prevent psychological problems. 


\section{Discussion}

As reflected by the first part of this review, there is considerable variation in the way resilience is currently being understood, defined and measured both within animal and human studies.

In animals, the main challenge is to understand how one can identify a "resilient" animal and how this relates to resilience in humans. Most animal studies of resilience identify resilient animals based on the absence of stress-related behavioral features. However, when assessing an animal's behavior, one should evaluate both the absence of stress-related behavioral features or biological markers, and the presence of adaptive behaviors or markers. For an overview of studies that have started to identify such adaptive behavioral, neural and molecular mechanisms, the reader is referred to Pfau and Russo ${ }^{85}$ and Russo et al. ${ }^{86}$. Furthermore, only few animal studies have incorporated a baseline behavioral or physiological measure in order to assess dynamic changes over time. Using cross-sectional designs, recent studies identified distinct molecular signatures that were associated with adaptive or maladaptive behavioral responses to stress ${ }^{87-89}$. Adding a baseline measurement to such approaches would be highly valuable in identifying baseline differences between animals along with the pattern of change from pre- to post-stressor within the same animal. Linking such markers with differential susceptibility to a stressor will further yield valuable insights into the molecular mechanisms of resilience which, in turn, will more efficiently lead to the identification of (a combination of) predictive biomarkers of resilience. Lastly, researchers need to carefully reflect upon their animal model (e.g., sex, strain, and age), phenotypes of interest and experimental designs (e.g., timing, duration, and type of stressor) ${ }^{90}$ in order to fulfill different types of validities to make their tests and models translatable to the clinical situation ${ }^{91,92}$.

In humans, although conducting prospective studies has received increasing attention, most findings are still preliminary since replication is often lacking and most studies harbor low effect sizes and relatively small sample sizes. It is also important to note that (i) a wide variety of tools (which cannot be compared easily) are currently being used to measure "resilience" of which the validity needs critical examination, (ii) the majority of resilience studies focuses on PTSD-related outcomes instead of positive outcome measures, and (iii) most of the studies which fulfilled the rather strict inclusion and exclusion criteria were conducted in military cohorts. While such samples provide a unique opportunity to study the effects of trauma exposure, they are also subjected to a natural limitation since sampling bias cannot be excluded. 
For future studies, researchers are encouraged to include a wider range of assessments when aiming to study and measure resilience in order to obtain a more reliable and objective operationalization of this concept. Using several techniques such as ESM ${ }^{93}$, in-person interviews combined with self-evaluations or targeted questionnaires and physiological measures including heart rate and blood pressure will allow one to obtain a more global picture on general psychological and physiological health. Furthermore, ESM might help to better understand processes such as inoculation during which individuals may develop resilience through repeated stress exposure. When possible, this, again, should be embedded within large-scale longitudinal studies since these allow tracking the stability of one's mental health over a specific time period. Moreover, and in order to facilitate extrapolation to the general population, there is a strong need for the inclusion of women in these studies, which is currently underdone ${ }^{94}$. This is crucial when knowing that women are more likely than men to develop stress-related mental disorders. Although this is a much broader phenomenon within science, both in animal and human research, women are even less likely to be included in studies using military cohorts in which they are underrepresented. An increase in the number of studies focusing on this population will undoubtedly enhance our current understanding of their stress responsiveness, health care and gender-specific needs. Lastly, in the context of searching for biological underpinnings or biomarkers that reliably predict differential susceptibility to future stress or psychological and biological resilience mechanisms, exploring the potential to combine several predictors, e.g., genetic, epigenetic, and/or imaging data on an individual basis is strongly encouraged. This further calls for more large-scale brain imaging studies in order to identify the brain regions involved in stress resilience.

Finally, establishing alternative strategies aiming to install positive emotions and improve cognitive abilities, social interactions, feelings of purpose and meaning of life along with physical health have obtained scientific evidence for their benefits in increasing one's global mental health, whilst the biological underpinning of these effects have remained understudied thus far. Gaining a deeper understanding of the underlying mechanisms of each of these strategies will undoubtedly aid in developing new treatment options for stress-related disorders such as PTSD. 


\section{Author contributions}

$\mathrm{CS}$ and L-KP contributed equally to this manuscript. GAJ contributed to the interpretation of the obtained data. NS, GAJ, NY, LdN, and SG reviewed the manuscript and provided comments/suggestions. BR reviewed the manuscript, provided comments/suggestions, and is the corresponding author.

\section{Funding}

Acknowledge personal funding of GAJ; King Faisal University, Saudi Arabia. 


\section{References}

1. Wittchen HU, Jacobi F, Rehm J, Gustavsson A, et al. The size and burden of mental disorders and other disorders of the brain in Europe 2010. Eur Neuropsychopharmacol. 2011;21(9):655-79.

2. Steinert C, Hofmann M, Kruse J, Leichsenring F. Relapse rates after psychotherapy for depression - stable long-term effects? A metaanalysis. JAffect Disord. 2014;168:107-18.

3. Kalisch R, Baker DG, Basten U, et al. The resilience framework as a strategy to combat stress-related disorders. Nat Hum Behav. 2017;1(11):784.

4. Rutten BP, Hammels C, Geschwind N, et al. Resilience in mental health: linking psychological and neurobiological perspectives. Acta Psychiatr Scand. 2013;128(1):3-20.

5. Fletcher D, Sarkar M. Psychological resilience: a review and critique of definitions, concepts, and theory. Eur Psychol. 2013;18(1):12-23.

6. Chen RJ, Kelly G, Sengupta A, et al. MicroRNAs as biomarkers of resilience or vulnerability to stress. Neuroscience. 2015;305:36-48.

7. Hodes GE, Pfau ML, Leboeuf M, et al. Individual differences in the peripheral immune system promote resilience versus susceptibility to social stress. Proc Natl Acad $S_{C i}$ USA. 2014;111(45):16136-41.

8. Kim JG, Jung HS, Kim KJ, Min SS, Yoon BJ. Basal blood corticosterone level is correlated with susceptibility to chronic restraint stress in mice. Neurosci Lett. 2013;555:137-42.

9. Magalhaes R, Barriere DA, Novais A, et al. The dynamics of stress: a longitudinal MRI study of rat brain structure and connectome. $\mathrm{Mol}$ Psychiatry. 2017;23(10):1998-2006.

10. Rasmussen DD, Crites NJ, Burke BL. Acoustic startle amplitude predicts vulnerability to develop post-traumatic stress hyper-responsivity and associated plasma corticosterone changes in rats. Psychoneuroendocrinology. 2008;33(3):28291.

11. Tse YC, Montoya I, Wong AS, et al. A longitudinal study of stress-induced hippocampal volume changes in mice that are susceptible or resilient to chronic social defeat. Hippocampus. 2014;24(9):1120-1128.

12. Macfarlane LA, Mrphy PR. MicroRNA: biogenesis, function and role in cancer. Curr Genomics. 2010;11(7):537-561.

13. Van Rooij S, Kennis M, Sjouwerman R, Van Den Heuvel M, Kahn R, Geuze E. Smaller hippocampal volume as a vulnerability factor for the persistence of post-traumaticstress disorder. PsycholMed.2015;45(13):2737-46.

14. van Wingen GA, Geuze E, Caan MW, et al. Persistent and reversible consequences of combat stress on the mesofrontal circuit and cognition. Proc Natl Acad Sci USA. 2012;109(38):15508-13.

15. Kennis M, Van Rooij S, Van Den Heuvel M, Kahn R, Geuze E. Functional network topology associated with posttraumatic stress disorder in veterans. NeuroImage Clin. 2016;10:302-9.

16. Van Rooij SJ, Kennis M, Vink M, Geuze E. Predicting treatment outcome in PTSD: a longitudinal functional MRI study on trauma- unrelated emotional processing. Neuropsychopharmacology. 2016;41(4):115665.

17. van Zuiden M, Geuze E, Willemen HL, et al. Pre-existing high glucocorticoid receptor number predicting development of posttraumatic stress symptoms after military deployment. Am JPsychiatry. 2011;168(1):8996.

18. Smid GE, van Zuiden M, Geuze E, Kavelaars A, Heijnen CJ, Vermetten E. Cytokine production as a putative biological mechanism underlying stress sensitization in high combat exposed soldiers. Psychoneuroendocrinology. 2015;51:534-46.

19. Reijnen A, Geuze E, Vermetten E. Individual variation in plasma oxytocin and vasopressin levels in relation to the development of combatrelated PTSD in a large military cohort. $J$ Psychiatr Res. 2017;94:88-95.

20. Rutten BPF, Vermetten E, Vinkers $\mathrm{CH}$, et al. Longitudinal analyses of the DNA methylome in deployed military servicemen identify susceptibility loci for post-traumatic stress disorder. Mol Psychiatry. 2017;23(5):1145- 
56.

21. Schur RR, Boks MP, Rutten BPF, et al. Longitudinal changes in glucocorticoid receptor exon $1 \mathrm{~F}$ methylation and psychopathology after military deployment. Transl Psychiatry.2017;7(7):e1 181.

22. Eraly SA, Nievergelt CM, Maihofer AX, et al. Assessment of plasma C-reactive protein as a biomarker of posttraumatic stress disorder risk. JAMA Psychiatry. 2014;71(4):423-31.

23. Breen MS, Maihofer AX, Glatt SJ, et al. Gene networks specific for innate immunity define post-traumatic stress disorder. Mol Psychiatry. 2015;20(12):1538-45.

24. Torshizi AD, Wang K. Deconvolution of transcriptional networks in post-traumatic stress disorder uncovers master regulators driving innate immune system function. $S_{C i}$ Rep. 2017;7(1):14486.

25. Steudte-Schmiedgen S, Stalder T, Schonfeld $S$, et al. Hair cortisol concentrations and cortisol stress reactivity predict PTSD symptom increase after trauma exposure during military deployment.

Psychoneuroendocrinology. 2015;59:123-33.

26. Trautmann S, Muehlhan M, Kirschbaum C, Wittchen HU, Hofler M, Stalder T, et al. Biological stress indicators as risk markers for increased alcohol use following traumatic experiences. Addict Biol. 2018;23(1):281-90.

27. Clark R, DeYoung CG, Sponheim SR, et al Predicting post-traumatic stress disorder in veterans: interaction of traumatic load with COMT gene variation. J Psychiatr Res. 2013;47(12):1849-56.

28. Wald I, Degnan KA, Gorodetsky E, et al. Attention to threats and combat-related posttraumatic stress symptoms: prospective associations and moderation by the serotonin transporter gene. JAMA Psychiatry. 2013;70(4):401-8.

29. Hoge CW, Castro CA, Messer SC, McGurk D, Cotting DI, Koffman RL. Combat duty in Iraq and Afghanistan, mental health problems, and barriers to care. NEnglJMed. 2004;351(1):13-22.

30. American Psychiatric Association. Diagnostic and Statistical Manual of Mental Disorders (DSM-5 (R). Washington, DC: American Psychiatric Pub (2013).
31. King LA, King DW, Vogt DS, Knight J, Samper RE. Deployment Risk and Resilience Inventory: a collection of measures for studying deployment- related experiences of military personnel and veterans. Milit Psychol. 2006;18(2):89-120.

32. Kolassa I-T, Kolassa S, Ertl V, Papassotiropoulos A, Dominique J-F. The risk of posttraumatic stress disorder after trauma depends on traumatic load and the catechol-O-methyltransferase Val158Met polymorphism. Biol Psychiatry. 2010;67(4):304-8.

33. Zuj DV, Palmer MA, Lommen MJ, Felmingham KL. The centrality of fear extinction in linking risk factors to PTSD: a narrative review. Neurosci Biobehav Rev. 2016;69:15-35.

34. Hayes JP, Logue MW, Reagan A, et al. COMT Val158Met polymorphism moderates the association between PTSD symptom severity and hippocampal volume. J Psychiatry Neurosci. 2017;42(2):95-102.

35. Uddin M, Galea S, Chang SC, et al. Gene expression and methylation signatures of MAN2C1 are associated with PTSD. Dis Markers.2011;30(3):111-21.

36. Mehta D, Klengel T, Conneely KN, et al. Childhood maltreatment is associated with distinct genomic and epigenetic profiles in posttraumatic stress disorder. Proc Natl Acad Sci USA. 2013;110(20):8302-8307.

37. Vinkers CH, Kalafateli AL, Rutten BP, et al. Traumatic stress and human DNA methylation: a critical review. Epigenomics. 2015;7(4):593-608.

38. Zannas AS, Provencal N, Binder EB. Epigenetics of posttraumatic stress disorder: current evidence, challenges, and future directions. Biol Psychiatry. 2015;78(5):327-35.

39. Ratanatharathorn A, Boks MP, Maihofer AX, et al. Epigenome-wide association of PTSD from heterogeneous cohorts with a common multi-site analysis pipeline. Am J Med Genet B Neuropsychiatr Genet. 2017;174(6):619-30.

40. Baker DG, Nievergelt CM, OConnor DT. Biomarkers of PTSD: neuropeptidesand immune signaling. Neuropharmacology. 2012;62(2):663-73.

41. McEwen BS, Gray JD, Nasca C. 60 years of 
neuroendocrinology: Redefining

neuroendocrinology: stress, sex and cognitive and emotional regulation. J Endocrinol. 2015;226(2):T67-T83.

42. Kabat-Zinn J. Mindfulness-based interventions in context: past, present, and future. Clin Psychol Sci Prac. 2006;10(2):14456.

43. Hölzel BK, Carmody J, Evans KC, et al. Stress reduction correlates with structural changes in the amygdala. Soc Cogn Affect Neurosci. 2010;5(1):11-7.

44. Singleton O, Holzel BK, Vangel M, Brach N, Carmody J, Lazar SW. Change in brainstem gray matter concentration following a mindfulness- based intervention is correlated with improvement in psychological wellbeing. Front Hum Neurosci. 2014;8:33.

45. Black DS, Cole SW, Irwin MR, Breen E, St Cyr NM, Nazarian N, et al. Yogic meditation reverses NF-kappaB and IRF-related transcriptome dynamics in leukocytes of family dementia caregivers in a randomized controlled trial. Psychoneuroendocrinology. 2013;38(3):348-55.

46. Lavretsky H, Epel ES, Siddarth P, Nazarian N, Cyr NS, Khalsa DS, et al. A pilot study of yogic meditation for family dementia caregivers with depressive symptoms: effects on mental health, cognition, and telomerase activity. Int J Geriatr Psychiatry.

2013;28(1):57-65.

47. Bhasin MK, Dusek JA, Chang BH, et al. Relaxation response induces temporal transcriptome changes in energy metabolism, insulin secretion and inflammatory pathways. PLoS ONE. 2013;8(5):e62817.

48. Goleman DJ, Schwartz GE. Meditation as an intervention in stress reactivity. JConsult Clin Psychol. 1976;44(3):456-66.

49. Jain S, Shapiro SL, Swanick S, et al. A randomized controlled trial of mindfulness meditation versus relaxation training: effects on distress, positive states of mind, rumination, and distraction. Ann Behav Med. 2007;33(1):11-21.

50. Tang YY, Ma Y, Wang J, et al. Short-term meditation training improves attention and self-regulation. Proc Natl Acad Sci USA. 2007;104(43):17152-6.
51. Ding X, Tang YY, Tang R, Posner MI Improving creativity performance by shortterm meditation. Behav Brain Funct. 2014;10(1):1-8.

52. Beck AT. Cognitive Therapy and the Emotional Disorders. New York, NY: International Universities Press (1976).

53. Singewald N, Schmuckermair C, Whittle N, Holmes A, Ressler K. Pharmacology of cognitive enhancers for exposure-based therapy of fear, anxiety and trauma-related disorders. Pharmacol Ther. 2015;149:150-90.

54. Pace-Schott EF, Verga PW, Bennett TS, Spencer RM. Sleep promotes consolidation and generalization of extinction learning in simulated exposure therapy for spider fear. $J$ Psychiatr Res. 2012;46(8):1036-44.

55. Iyadurai L, Blackwell SE, Meiser-Stedman R, et al. Preventing intrusive memories after trauma via a brief intervention involving Tetris computer game play in the emergency department: a proof- of-concept randomized controlled trial. Mol Psychiatry. 2018;23(3):674-682.

56. Padesky CA, Mooney KA. Strengths-based cognitive-behavioural therapy: a four-step model to build resilience. Clin Psychol Psychother. 2012;19(4):283-90.

57. Meichenbaum D. Stress Inoculation Training. Elmsford, NY: Pergamon Press (1985).

58. Gunnar MR, Frenn K, Wewerka SS, Van Ryzin MJ. Moderate versus severe early life stress: associations with stress reactivity and regulation in 10-12-year-old children. Psychoneuroendocrinology. 2009;34(1):6275.

59. Katz M, Liu C, Schaer M, et al. Prefrontal plasticity and stress inoculation-induced resilience. Dev Neurosci. 2009;31(4):293-9.

60. Parker KJ, Buckmaster CL, Schatzberg AF, Lyons DM. Prospective investigation of stress inoculation in young monkeys. Arch Gen Psychiatry. 2004;61(9):933-41.

61. Kashani F, Kashani P, Moghimian M, Shakour M. Effect of stress inoculation training on the levels of stress, anxiety, and depression in cancer patients. Iran J Nurs Midwifery Res. 2015;20(3):359-64.

62. Flaxman PE, Bond FW. A randomised worksite comparison of acceptance and 
commitment therapy and stress inoculation training. Behav Res Ther. 2010;48(8):816-20.

63. Penedo FJ, Dahn JR. Exercise and well-being: a review of mental and physical health benefits associated with physical activity. Curr Opin Psychiatry. 2005;18(2):189-93.

64. van Praag H, Christie BR, Sejnowski TJ, Gage FH. Running enhances neurogenesis, learning, and long-term potentiation in mice. Proc Natl Acad Sci USA. 1999;96(23):1342731.

65. Colcombe SJ, Kramer AF, Erickson KI, et al. Cardiovascular fitness, cortical plasticity, and aging. Proc Natl Acad Sci USA. 2004;101(9):3316-21

66. Erickson KI, Voss MW, Prakash RS, et al. Exercise training increases size of hippocampus and improves memory. Proc Natl Acad Sci USA. 2011;108(7):3017-22.

67. Wichers M, Peeters F, Rutten BP, et al. A timelagged momentary assessment study on daily life physical activity and affect. Health Psychol. 2012;31(2):135-44.

68. Manjunath NK, Telles S. Improved performance in the Tower of London test following yoga. Indian J Physiol Pharmacol. 2001;45(3):351-4.

69. Netz Y, Lidor R. Mood alterations in mindful versus aerobic exercise modes. J Psychol. 2003;137(5):405-19.

70. Dale LP, Mattison AM, Greening K, Galen G, Neace WP, Matacin ML. Yoga workshop impacts psychological functioning and mood of women with self-reported history of eating disorders. Eat Dis. 2009;17(5):422-34.

71. Robert McComb JJ, Tacon A, Randolph P, Caldera Y. A pilot study to examine the effects of a mindfulness-based stress-reduction and relaxation program on levels of stress hormones, physical functioning, and submaximal exercise responses. J Altern Complement Med. 2004;10(5):819-27.

72. Danucalov MA, Simoes RS, Kozasa EH, Leite JR. Cardiorespiratory and metabolic changes during yoga sessions: the effects of respiratory exercisesand meditation practices. Appl Psychophysiol Biofeedback.2008;33(2):77-81.

73. Telles S, Reddy SK, Nagendra HR. Oxygen consumption and respiration following two yoga relaxation techniques. Appl Psychophysiol
Biofeedback. 2000;25(4):221-7.

74. Cohen S. Social relationships and health. Am Psychol. 2004;59(8):676-84.

75. Fratiglioni L, Paillard-Borg S, Winblad B. An active and socially integrated lifestyle in late life might protect against dementia. Lancet Neurol. 2004;3(6):343-53.

76. Lorenz RA, Gooneratne N, Cole CS, Kleban MH, Kalra GK, Richards KC. Exercise and social activity improve everyday function in long- term care residents. Am J Geriatr Psychiatry. 2012;20(6):468-76.

77. Ozbay F, Johnson DC, Dimoulas E, Morgan III C, Charney D, Southwick S. Social support and resilience to stress: from neurobiology to clinical practice. Psychiatry. 2007;4(5):35-40.

78. Fried LP, Carlson MC, Freedman M, et al. A social model for health promotion for an aging population: initial evidence on the Experience Corps model. J Urban Health. 2004;81(1):64-78.

79. Carlson MC, Kuo JH, Chuang Y-F, et al. Impact of the Baltimore Experience Corps Trial on cortical and hippocampal volumes. Alzheimers Demen. 2015;11(11):1340-8.

80. Pinquart M. Creating and maintaining purpose in life in old age: a meta-analysis. Ageing Int. 2002;27(2):90-114.

81. Pearson MR, Brown DB, Bravo AJ, Witkiewitz K. Staying in the moment and finding purpose: the associations of trait mindfulness decentering, and purpose in life with depressive symptoms, anxiety symptoms, and alcohol-related problems. Mindfulness. 2014;6(3):645-53.

82. Orbach I, Mikulincer M, Gilboa-Schechtman E, Sirota P. Mental pain and its relationship to suicidality and life meaning. Suicide Life Threat Behav. 2003;33(3):231-41.

83. Lee J, Cho D, Suh YJ. Purpose and meaning in life and job satisfaction among the aged. Int J Aging Hum Dev. 2017;85(4):377-402.

84. Lyon DE, Younger JB. Purpose in life and depressive symptoms in persons living with HIV disease. J Nurs Scholarsh. 2001;33(2):129-33.

85. Pfau ML, Russo SJ. Peripheral and central mechanisms of stress resilience. Neurobiol Stress. 2015;1:66-79.

86. Russo SJ, Murrough JW, Han MH, Charney 
DS, Nestler EJ. Neurobiology of resilience. Nat Neurosci. 2012;15(11):1475-84.

87. Krishnan V, Han M-H, Graham DL, et al. Molecular adaptations underlying susceptibility and resistance to social defeat in brain reward regions. Cell. 2007;131(2):391-404.

88. Friedman AK, Walsh JJ, Juarez B, et al. Enhancing depression mechanisms in midbrain dopamine neurons achieves homeostatic resilience. Science. 2014;344(6181):313-9.

89. Wang M, Perova Z, Arenkiel BR, Li B. Synaptic modifications in the medial prefrontal cortex in susceptibility and resilience to stress. J Neurosci. 2014;34(22):7485-92.

90. Scharf SH, Schmidt MV. Animal models of stress vulnerability and resilience in translational research. Curr Psychiatry Rep. 2012;14(2):159-65.

91. Belzung C, Lemoine M. Criteria of validity for animal models of psychiatric disorders: focus on anxiety disorders and depression. Biol Mood Anxiety Disord. 2011;1(1):9.

92. Stewart AM, Kalueff AV. Developing better and more valid animal models of brain disorders. Behav Brain Res. 2015;276:28-31.

93. Verhagen SJ, Hasmi L, Drukker M, van Os J, Delespaul PA. Use of the experience sampling method in the context of clinical trials. Evid Based Mental Health. 2016;19(3):86-9.

94. Gururajan A, Kos A, Lopez JP. Preclinical stress research: where are we headed? An early career investigators perspective. Stress. 2018;21(5):384 




\subsection{Summary}

In Chapter 2, the longitudinal risk for the incidence of clinical psychosis was assesses in a general population sample in relation to preceding psychosis risk states and DSM-IV diagnoses of non-psychotic mental disorders (mood disorders, anxiety disorders, alcohol use disorders, and drug use disorders). Estimated hazard ratio suggested that mood disorders, drug use disorders, and psychosis high-risk state are important determinants for increased risk for clinical psychosis. However, given the low prevalence of the high-risk state, the fraction that could be prevented if the high-risk state was eliminated (population attributable fraction) was comparatively low. Successful psychosis prevention approaches might benefit from focusing on broad psychopathology.

In Chapter 3, I evaluated to what degree the association between risk factors commonly associated with psychosis spectrum disorder (PSD; i.e. environmental and proxy genetic factors) and psychosis expression in the general population is contingent on the presence of other dimensions of psychopathology (i.e. affective dysregulation, negative symptoms, and cognitive alterations). The results show a dose-response relationship between risk factors associated with PSD and psychosis expression, with greater odds for psychosis expression being associated with the exposure to a greater number of risk factors. Furthermore, in support of the multidimensional approach, the effect that risk factors had on PE was contingent on the presence of the other symptom dimensions, especially affective dysregulation.

In Chapter 4, I introduce one of the epidemiological datasets that were used in this dissertation. The TwinssCan project is a longitudinal general population twin cohort, which recruited from the East Flanders Prospective Twin Survey. The review highlights important findings on the contribution of environmental and genetic factors on subclinical expression of psychosis and affective phenotypes, focusing on macro (e.g. monthly) as well as micro (e.g. momentary) level psychopathological changes. Furthermore, applying novel experimental tools, studies conducted in this cohort evaluated the role of neurocognitive processes such as salience attribution and sensitivity to social defeat in the pathway to mental ill health.

In Chapter 5, one of the studies conducted in the TwinssCan sample is presented. Possible due to different methodological approaches, previous studies found inconsistent results on whether speech illusions during the white noise task are associated with subclinical expression of psychotic symptoms in the general population. Therefore, I tested the association between speech illusion and measurements for subclinical expression of psychotic symptoms following two different approaches. The results suggest, that contrary to findings derived from clinical 
samples, speech illusion during the white noise task are not associated with subclinical expression of psychotic symptoms in the general population.

Chapter 6 presents a study testing polygenic risk score for schizophrenia (PRS-SCZ) for an interaction with early and late stressors (childhood adversity and minor daily-life stressors) in association with dynamic pluripotent mental processes in the largest ever EMA data collected from the TwinssCan project. The study provided evidence that childhood adversity and early lifetime stressors pleiotropically affect emotional dysregulation and subtle psychosis expression in daily life. Furthermore, while PRS-SCZ does not interact with daily-life stressors, it interacts with childhood adversity on pluripotent psychopathology. The findings help to understand person-specific effects of the complex interactions between environmental and genetic factors on nonspecific health outcomes.

In Chapter 7 the independent and joint effects of molecular genetic liability and environmental exposures in schizophrenia was tested in a large international case-control cohort with patients diagnosed with schizophrenia. Several environmental exposures, as well as polygenic risk score for schizophrenia, were associated with case-status. The study indicated a positive additive interaction between PRS-SCZ and several environmental exposures (i.e. lifetime regular cannabis use and early-life adversities; sexual abuse, emotional abuse, emotional neglect, and bullying). The findings suggest that some individuals may only develop a diagnosis of schizophrenia if both the genetic and nongenetic exposures (i.e. emotional and sexual abuse) are present.

In Chapter 8 the exposome score for schizophrenia (an aggregate score of environmental liability for schizophrenia) was for the first time estimated and validated by means of predictive modeling approach in two large independent datasets. Exposome scores for schizophrenia were estimated using different models; logistic regression, Gaussian Naïve Bayes, the LASSO and Ridge penalized classification models. Additionally, an exposome score based on meta-analyses and a simple sum-score of exposures were estimated. The findings demonstrate that a simple score based on logistic regression, which takes into account of the dependent effect of various exposures, is best equipped to assess environmental liability for schizophrenia in an independent test dataset. The exposome score for schizophrenia significantly differentiated between cases, unaffected siblings, and healthy controls in the independent test sample.

Chapter 9 tested whether recent stressful life events interacted with indicators of genetic liability, polygenic risk score for schizophrenia, and environmental liability, exposome score 
for schizophrenia on health outcome in the general population. Genetic and environmental liability was associated with poorer health outcomes, especially mental health. The exposome score moderated the association of recent stressful life events with mental and physical health, while polygenic risk did not. The results were confirmed by several sensitivity analyses.

Chapter 10 investigated the contribution of the polygenic risk score and the exposome score for schizophrenia to psychosis expression across the spectrum. Genomic and exposomic liability were associated with a diagnosis of schizophrenia and schizotypy in healthy controls and unaffected siblings. Furthermore, genomic liability moderated the effect of exposomic liability on case-status and schizotypy in healthy controls and unaffected siblings. The results support the conceptual framework of an etiological continuity across psychosis spectrum. 



\subsection{Impact paragraph}

Schizophrenia contributes to $€ 93.9$ billion, one of the largest portion of European healthcare costs due to mental disorders ${ }^{1}$. In the Netherlands specifically, it accounts for $0.11 \%$ of the GDP with an annual direct cost of $\$ 774$ million $^{2}$. Furthermore, schizophrenia is associated with decreased functioning, low quality of life, and is among the top five leading causes of disability adjusted life years (DALYs) in the age-group ranging from 15 to 44 years ${ }^{3}$. Therefore, there is an urgent need for a breakthrough in prevention, diagnosis, and management of schizophrenia, which can be achieved through better understanding of pathoetiology.

The studies presented in this thesis, closely tied to the goals of the national research agenda "Nationale Wetenschapsagenda" in the areas of "Gezondheidszorgonderzoek, preventie en behandeling" and "Personalised medicine: uitgaan van het individu", contributes greatly to research on the pathoetiology of psychosis spectrum. The knowledge utilized in this thesis can increase the efficient use of public health care tools and eventually help us to decrease disorder-related burden and costs.

The thesis highlights the importance of the investigation of the whole network of environmental and genomic exposures. The studies underline the opportunity centering on modifiable environmental factors to improve population-based mental health outcomes. Furthermore, presenting the first studies indicating gene-environment interaction $(\mathrm{GxE})$ for psychosis spectrum using exposomic and molecular genomic measures, the studies in this thesis initiates great opportunity for subsequent research on $\mathrm{GxE}$ and highlight the importance for the shift from diagnostic category based research towards the investigation of psychosis spectrum.

Refining the toolbox for multimodal research in psychiatry, this thesis generates a knowledgebase for national and international scientific programs of the multilevel etiology of psychiatry. The exposome score applied in this project can be used to reduce the bias due to environmental confounding and increase causal inference in other observational, clinical, or experimental studies of psychotic disorders across Europe, particularly the Netherlands. In future studies, testing the exposome paradigm in the context of biological mechanisms and epigenomic markers will contribute to the global efforts toward a better understanding of psychotic disorders and provide candidate biological targets and processes for translational research. Further, as exposomic and genomic liability does not only play a critical role in psychotic disorders but a range of mental disorders ${ }^{4-7}$, this approach may be applied to other 
large population cohorts to investigate GxE such as the NIH's "All of US" and emerging exposomic research endeavors ${ }^{8}$.

The integration of psychiatric epidemiology and molecular genetic markers offers potential benefits for risk stratification and screening toward personalized medicine. Findings may aid the global efforts toward a diagnostic classification that should ideally be driven by neurobiological substrates and etiologic mechanisms, which in turn would accelerate the delivery of improved health outcomes through more accurate diagnosis, preventive measures, and treatment.

This research line can greatly contribute to the reputation and the impact of the regional academic activities in mental health. Results from the studies presented in this thesis were already picked up by the general public and will be discussed with patient organizations. It will inform patients, general public, policy makers and disseminate the concept that the expression of genetic vulnerability depends on exposure to environmental factors. Eventually, this will greatly help patients in their understanding of their personal background of experiencing psychosis spectrum disorder. 


\section{References}

1. Gustavsson A, Svensson M, Jacobi F, et al. Cost of disorders of the brain in Europe 2010. Eur Neuropsychopharmacol. 2011;21(10):718-779.

2. Chong HY, Teoh SL, Wu DB-C, Kotirum S, Chiou C-F, Chaiyakunapruk N. Global economic burden of schizophrenia: asystematic review. Neuropsychiatric disease treatment. 2016;12:357.

3. Murray CJ, Vos T, Lozano R, et al. Disabilityadjusted life years (DALYs) for 291 diseases and injuries in 21 regions, 1990-2010: a systematic analysis for the Global Burden of Disease Study 2010. The lancet. 2012;380(9859):2197-2223.

4. Kendler KS, Eaves LJ, Loken EK, et al. The impact of environmental experiences on symptoms of anxiety and depression across the life span. Psychological Science. 2011;22(10):1343-1352.

5. Rutten BP, Hammels C, Geschwind N, et al Resilience in mental health: linking psychological and neurobiological perspectives. Acta Psychiat Scand. 2013;128(1):3-20.

6. Rutten BP, Vermetten E, Vinkers $\mathrm{CH}$, et al. Longitudinal analyses of the DNA methylome in deployed military servicemen identify susceptibility loci for post-traumatic stress disorder. Molecular psychiatry. 2018;23(5):1145-1156.

7. Cross-Disorder Group of the Psychiatric Genomics Consortium. Identification of risk loci with shared effects on five major psychiatric disorders: a genome-wide analysis. The Lancet. 2013;381(9875):1371-1379.

8. Stingone JA, Buck Louis GM, Nakayama SF, et al. Toward greater implementation of the exposome research paradigm within environmental epidemiology. Annual review of public health. 2017;38:315-327 



\subsection{Acknowledgment}

First of all, I would like to thank my supervisor team: Dear Jim, I am impressed by your ability to listen, learn and execute. I am inspired by your enthusiasm for our research, which will impact me throughout my career. Dear Bart, I would like to thank you for your guidance throughout my MSc and the $\mathrm{PhD}$ programs. Your optimism and drive deeply impressed me and helped me through the ups and downs of the PhD. Dear Sinan, last but not least, your supervision and guidance are the reasons I can proudly look at my thesis and proudly force people to read it. Pushing me to my limits, you helped me reach the top of the mountains (and sometimes insanity;)). Your support made this piece of work possible. As I'm currently doing a postdoc with you, I will leave some space for further evaluation, so be nice!

Dear colleagues, thank you for the lunches, enjoyable day-outs, and support. Special thanks to Trees, Truda, Jolanda, Ron, and Jolien. They keep the machine running. Thank you to the old PhD students (Stijn, Iris, Thomas, and Hening) and the new PhD students (Christian, Nikita, Sofie, Mary, Simone, Sam, Stella, and Maud). Special thanks to Simone, you always have good advice, a helping hand, and time for a nice little break! Thanks to Sam, Stella, and Maud for the fun, food and beauty ;) Thanks to my roommates Mary and Stijn for the nice little interruptions. Dear Ehsan, thank you for believing in me and encouraging me to pursuit a PhD. I would not be here if it wasn't for you. Dear Gunter, thanks for the collaboration, I enjoyed working together. A great thanks also goes to my coauthors and their helpful remarks and feedback.

Dear friends and family, Liebe Freunde und Familie,

I would like to thank my friends and beloved roommates who joined me on this path during the Maastricht years. Dear Clara, Kim, and Chiao, thanks for the talks and dinners, watching educational television, and conversations about randomness. Dear Selma, thanks for joining me in making fantastic pralines ;). Dear Hannan and Nathalie, thanks for the humor and silliness. Nathalie, I am very happy that Hannan convinced me to let you move in with us ;) Thanks for the midnight conversations!

Thanks to all the friends who joined me for dinners and creative adventures. Dear Sam, Ste and Tin, let's have avocado pasta at 7pm this time, ok? :) Dear Kwinten, thanks for the coffeeS, cooking, and beer making. I know we will follow this up with many more adventures. 
Thanks to all the friends from dinner clubs (but not to those who are not my $\mathrm{fb}$ friends;)) and to all the people challenging and encouraging me throughout these years!

Thanks to all my friends around the world. Liebe Sim, danke für deine Unterstützung und die Hilfe, um mein grauenhaftes Englisch in etwas Passables zu verwandeln ;) Liebe Amy und Bela, ihr wisst, dass ihr für immer in meinem Herzen sein werdet. Jedes Mal wenn ich euch sehe, bin ich voll mit Liebe und Glückseligkeit. Ich freue mich schon auf das nächste Mal, wenn ich euch sehe!

Ganz wichtig, vielen Dank an meine Familie, ohne euch würde ich nicht hier stehen. Auch wenn ihr noch nicht verstanden habt, wie unglaublich lustig und musikalisch ich bin, weiß ich, dass ich mich immer auf euch verlassen kann. Ohne euch, wäre ich nicht die Person, die ich heut bin. Liebe Charly, ich bin unglaublich froh, dass auch Teil von dir in diesem Buch im Titelbild verewigt ist. 




\subsection{Curriculum Vitae}

Lotta-Katrin Pries was born on March $6^{\text {th }}, 1990$ in Bergisch Gladbach, Germany. She graduated from Hansa-Gymnasium Köln in 2010 and she attended her Bachelor's degree in Psychology (BSc) at Maastricht University from 2011 to 2014. In the third year, she took the opportunity to follow an honors program (Marble, Research based learning), conducting a study into: "Directed Forgetting: Differences in the Strength of Memory-Traces" at Dalhousie University, Canada. Following this she enrolled in a two-year Master of Science in Cognitive and Clinical Neuroscience (specialization psychopathology) program at Maastricht University, which she graduated from in 2016. Immediately after, Lotta started a PhD program at the School for Mental Health and Neuroscience in the Department of Psychiatry and Neuropsychology at Maastricht University. The focus of this project was to investigate the interplay between environmental load (e.g. childhood trauma) and genetic load (polygenic risk scores for schizophrenia and other mental disorders). In 2020, she started as a postdoc at the same department. 



\subsection{List of Publications}

\section{Peer reviewed}

van Os J, Hasmi L, Pries L-K, ten Have M, de Graaf R, van Dorsselaer S, ... Rutten BPF, Guloksuz S (manuscript). Closing in on the onset of psychotic disorder: Evidence that a small fraction of incident non-psychotic disorder becomes 'psychotic' through a distinct clinical, cognitive and genetic profile.

Pries L-K* Erzin $\mathrm{G}^{*}$ van Os J, ten Have M, de Graaf R, van Dorsselaer S, ... Rutten BPF, Guloksuz S (2020). Predictive performance of exposome score for schizophrenia in the general population. Schizophr Bull, doi: 10.1093/schbul/sbaa170

Henquet C, van Os J, Pries L-K, Rauschenberg C, Delespaul P, Kenis G, ... Rutten BPF, Guloksuz S (2020). A Replication study of JTC bias, genetic liability for psychosis and delusional ideation. Psychological Medicine, doi:

10.1017/S0033291720003578

Pries L-K*, Dal Ferro GA*, van Os J, Delespaul P, Kenis G, Lin BD, ... Rutten BPF, Guloksuz S (2020). Examining the independent and joint effects of genomic and exposomic liabilities for schizophrenia across the psychosis spectrum.

Epidemiology and Psychiatric Sciences, doi: 10.1017/S2045796020000943

Marsman A, Pries L-K, ten Have M, de Graaf R, van Dorsselaer S, Bak M, ... Rutten BPF, Guloksuz S, van Os J (2020). Do current measures of polygenic risk for mental disorders contribute to population variance in mental health? Schizophr Bull, sbaa086, https://doi.org/10.1093/schbul/sbaa086

Pries L-K, van Os J, ten Have M, de Graaf R, van Dorsselaer S, Bak M, ... Rutten BPF, Guloksuz S (2020). Association of recent stressful life events with mental and physical health in the context of genomic and exposomic liability for schizophrenia. JAMA psychiatry, doi:10.1001/jamapsychiatry.2020.2304

Rauschenberg C, Reininghaus U, ten Have M, de Graaf R, van Dorsselaer S, Simons CJP ... Pries LK, Guloksuz S ... van Os J (2020). The jumping to conclusions reasoning bias as a cognitive factor contributing to psychosis progression and persistence: findings from NEMESIS-2. Psychol Med, 1-8, doi: 10.1017/S0033291720000446

Guloksuz S $^{*}$ Pries L-K* ten Have M, de Graaf R, van Dorsselaer S, Klingenberg B, ... Rutten BPF, van Os J (2020). Association of preceding psychosis risk states and non-psychotic mental disorders with incidence of clinical psychosis in the general population: a prospective study in the NEMESIS-2 cohort. World Psychiatry, 199-205. doi: 10.1002/wps.20755.

Authors had equal contribution 
Pries L-K, Klingenberg B, Menne-Lothmann C, Decoster J, van Winkel R, Collip D, ... Rutten BPF, van Os J, Guloksuz S (2020). Polygenic Liability for Schizophrenia and Childhood Adversity Influences Daily-Life Emotion Dysregulation and Psychosis Proneness. Acta Psychiatrica Scandinavica, 1-11, doi: 10.1111/acps. 13158

Pries L-K, Snijders C, Menne-Lothmann C, Decoster J, van Winkel R, Collip D, ... Guloksuz S, van Os J, Rutten BPF (2019). TwinssCan - Gene-environment interaction in psychotic and depressive intermediate phenotypes: risk and protective factors in a general population twin sample. Twin Res Hum Genet, 17, doi: $10.1017 /$ thg. 2019.96

Schepers E, Lousberg R, Guloksuz S, Pries L-K, Delespaul P, Kenis G, ... Rutten BPF, van Os J (2019). White noise speech illusions: a trait-dependent risk marker for psychotic disorder? Front Psychiatry, 676, doi: 10.3389/fpsyt.2019.00676

van Os J, Pries L-K, Delespaul P, Kenis G, Luyx JJ, Lin B, ... Rutten BPF, Guloksuz S (2019). Replicated evidence that endophenotypic expression of schizophrenia polygenic risk is greater in healthy siblings of patients compared to controls, suggesting gene-environment interaction. The EUGEI study. Psychol Med, 1-14, doi: 10.1017/S003329171900196X

Pries L-K, Lage-Castellanos A, Delespaul P, Kenis G, Luyx JJ, Lin B, ... Rutten BPF, van Os J, Guloksuz S (2019). Estimating Exposome Score for Schizophrenia Using Predictive Modeling Approach in Two Independent Samples: The Results from the EUGEI Study. Schizophr Bull, sbz054, https://doi.org/10.1093/schbul/sbz054

Guloksuz S*, Pries L-K*, Delespaul P, Kenis G, Luyx JJ, Lin B, ... Rutten BPF, van Os J (2019). Examining the independent and joint effects of molecular genetic liability and environmental exposures in schizophrenia: results from the EUGEI study. World Psychiatry, 173-182, doi: 10.1002/wps.20629

Snijders C*, Pries L-K*, Sgammeglia N, Youssef NA, de Nijs L, Guloksuz S, Rutten BPF (2018). Resilience against traumatic stress: current developments and future directions. Front Psychiatry, 676, doi: 10.3389/fpsyt.2018.00676

Guloksuz S, Rutten BPF, Pries L-K, ten Have M, de Graaf R, van Dorsselaer S, ... van Os J, Ioannidis J (2018). The Complexities of Evaluating the Exposome in Psychiatry: A Data-Driven Illustration of Challenges and Some Propositions for Amendments. Schizophr Bull, 1175-1179, doi: 10.1093/schbul/sby118

Radhakrishnan R, Guloksuz S, ten Have M, de Graaf R, van Dorsselaer S, Gunther N, Rauschenberg C, Reininghaus U, Pries L-K, Bak M, van Os, J (2018).

* Authors had equal contribution 
Interaction between environmental and familial affective risk impacts psychosis admixture in states of affective dysregulation. Psychol Med, 1879-1889, doi: $10.1017 /$ S0033291718002635.

Reininghaus U, Rauschenberg C, ten Have M, de Graaf R, van Dorsselaer S, Simons CJP, Gunther N, Pries L-K, Guloksuz S, Radhakrishnan R, Bak M (2018). Reasoning bias, working memory performance and a transdiagnostic phenotype of affective disturbances and psychotic experiences in the general population. Psychol Med, 1799-1809, doi: 10.1017/S0033291718002209

Pries L-K*, Guloksuz S*, ten Have M, de Graaf R, van Dorsselaer S, Gunther N, ... Rutten $\mathrm{BPF}$, van Os J (2018). Evidence that environmental and familial risks for psychosis additively impact a multidimensional subthreshold psychosis syndrome. Schizophr Bull, 710-719, doi: 10.1093/schbul/sby051

Guloksuz S, Pries L-K, van Os J (2017). Application of network methods for understanding mental disorders: pitfalls and promise. Psychol Med, 2743-2752, doi: $10.1017 / S 0033291717001350$

Pries L-K, Guloksuz S, Menne-Lothmann C, Decoster J, van Winkel R, Collip D, .. Rutten BPF, van Os J (2017). White noise speech illusion and psychosis expression: An experimental investigation of psychosis liability. PloS one, e0183695, doi: 10.1371/journal.pone.0183695

Taylor TL, Cutmore L, Pries L-K (2017). Item-method directed forgetting: Effects at retrieval? Acta Psychol, 116-23, doi: 10.1016/j.actpsy.2017.12.004

\section{Book chapter:}

Pries L-K, GuloksuzS, Rutten BPF. Resilience in psychosis spectrum disorder. under review.

Pries L-K, Guloksuz S, Kenis G. DNA Methylation in Schizophrenia. In: Neuroepigenomics in Aging and Disease. 2017; 211-236. Springer International Publishing.

\section{Conference activity}

\section{Oral presentations:}

Pries L-K, Klingenberg B, Menne-Lothmann C, DecosterJ, van Winkel R, Collip D, ... Guloksuz S. Interaction Between Polygenic Liability for Schizophrenia and Childhood Adversity Influences Daily-Life Emotional Dysregulation and

Authors had equal contribution 
Psychosis Proneness. Society of biological psychiatry's annual meeting, New York, USA 30 April - 2 May 2020 (symposium)

Guloksuz S, Pries L-K, Rutten BPF, van Os J. Examining the role of the interaction between the exposome and genome in schizophrenia spectrum disorder: results from the EUGEI. Voorjaarscongres NVvP, Maastricht, The Netherlands 1-3 April 2020 (symposium)

Pries L-K, Guloksuz S, ten Have M, de Graaf R, van Dorsselaer S, Rutten BPF, van Os J. Testing the High Risk and Transition Framework in the General Population: Population-Based Measures of Risk and Transition for Psychosis 6-Year Longitudinal Follow-Up. Schizophrenia Bulletin. Congress of the Schizophrenia International Research Society, Orlando, USA 10-14 April 2019 (verbal presentation)

Pries L-K, Klingenberg B, Menne-Lothmann C, Decoster J, van Winkel R, Collip D, ... GuloksuzS. Polygenic Risk for Schizophrenia Moderates the Influence of Childhood Adversity on Daily-Life Emotional Dysregulation and Psychosis Proneness. Schizophrenia Bulletin. Congress of the Schizophrenia International Research Society, Orlando, USA 10-14 April 2019 (symposium)

Pries L-K, Guloksuz S, Menne-Lothmann C, Decoster J, van Winkel R, Collip D, ... van Os J. Does Polygenic Risk Score for Schizophrenia Moderate the Momentary Affective and Psychotic Reactions to Daily-life Stressors? Schizophrenia Bulletin. Congress of the Schizophrenia International Research Society, Florence, Italy 4-8 April 2018 (verbal presentation)

\section{Poster presentations:}

Pries L-K, van Os J, ten Have M, de Graaf R, van Dorsselaer S, Bak M, ... Rutten BPF, Guloksuz S. The Main and Interactive Effects of Adult Stressful Life Events with Genomic and Exposomic Liability for Schizophrenia on Mental and Physical health: A Prospective Cohort Study. Congress of the Schizophrenia International Research Society, Florence, Italy 4-8 April 2020

Pries L-K, Klingenberg B, Menne-Lothmann C, Decoster J, van Winkel R, Collip D, .. GuloksuzS. Polygenic risk for schizophrenia moderates the influence of childhood adversity on daily-life emotional dysregulation and psychosis proneness. Annual Research Day of the School for Mental Health and Neuroscience, Maastricht, the Netherlands 28 November 2018

Rauschenberg C, Reininghaus U, ten Have M, de Graaf R, van Dorsselaer S, Gunther N, Pries L-K, ... van Os J. Reasoning bias, working memory performance, and a transdiagnostic phenotype of affective disturbances and psychotic experiences in 
the general population. Congress of the Schizophrenia International Research Society, Florence, Italy 4-8 April 2018

Pries L-K, Guloksuz S, ten Have M, de Graaf R, van Dorsselaer S, Gunther N, ... van Os J. Evidence that environmental and familial risks for psychosis additively impact a multidimensional subthreshold psychosis syndrome. Annual Research Day of the School for Mental Health and Neuroscience, Maastricht, the Netherlands 30 November 2017

Pries L-K, Guloksuz S, Menne-Lothmann C, Decoster J, van Winke R, Collip D, ... van Os J. White noise speech illusions and psychosis expression in the general population: an experimental investigation of psychosis liability. World Congress of Psychiatry, Berlin, Germany 8-12 October 2017

Pries L-K, Pishva E, Kenis G, Decoster J, De Hert M, Delespaul P, ... Rutten BPF. Does physical activity moderate the impact of childhood trauma on the liability of mental illness? Dutch Neuroscience Meeting, Lunteren, The Netherlands 9-10 June 2016 


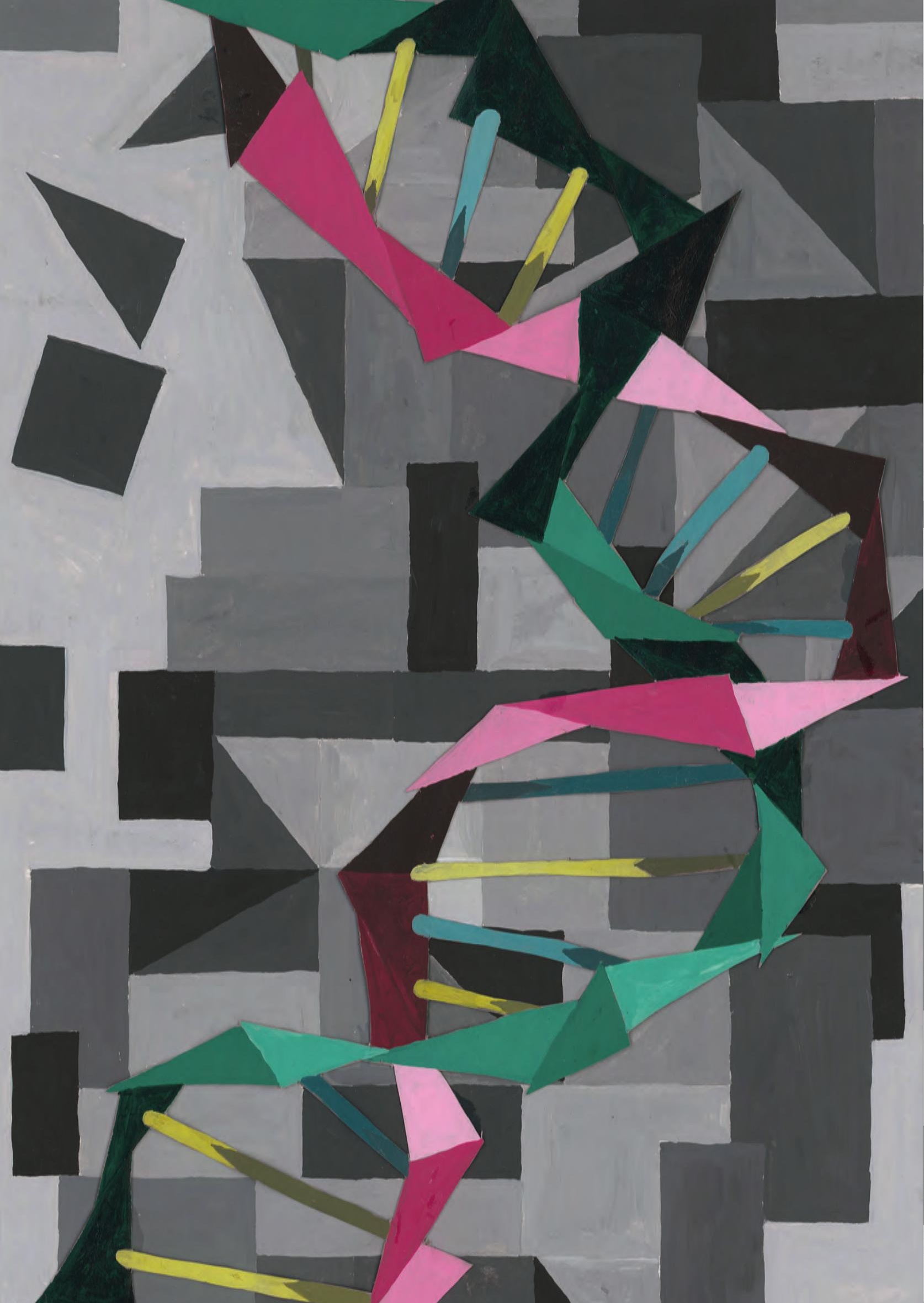

